\title{
29. PALEOMAGNETISM OF DEEP SEA DRILLING PROJECT LEG 85 SEDIMENTS: NEOGENE MAGNETOSTRATIGRAPHY AND TECTONIC HISTORY OF THE CENTRAL EQUATORIAL PACIFIC ${ }^{1}$
}

\author{
Norbert Weinreich, Institut für Geophysik, Ruhr-Universität Bochum \\ and \\ Fritz Theyer, Hawaii Institute of Geophysics, University of Hawaii ${ }^{2}$
}

\begin{abstract}
On Leg 85,16 holes were cored at five sites. Thirteen of the holes were cored with the hydraulic piston corer (HPC) or the variable-length hydraulic piston corer (VLHPC) or both; the remainder were rotary drilled. Partially duplicating stratigraphic sections were successfully recovered by hydraulic piston coring at Sites 572 to 575 . Sub-bottom penetration was deepest (about $210 \mathrm{~m}$ ) at HPC Hole 575A, which bottomed in lower Miocene sediments. Penetration by hydraulic piston coring was limited at all sites not by the failure of the corer to stroke out but by the excessive force (overpull) necessary to retrieve the core barrel from the hole. The sediments recovered are relatively uniform siliceous-calcareous oozes to calcareous ooze-chalks. Paleomagnetic measurements were made at all stratigraphic levels, but magnetostratigraphic sequences could be resolved only for the Pleistocene-Pliocene and for brief upper, middle, and lower Miocene sections.

In the younger and less consolidated sediments, the declination often shows large-scale azimuthal rotations downcore. These smooth trends vary from core to core, indicating either rotation between the sediment and the core liner or the rotation of the core barrel during the coring or retrieval process. Thus, azimuthal orientation of the samples was impossible even though a Kuster azimuthal orientation tool was used during the hydraulic piston coring.

At all sites, the downhole shift from mainly siliceous to mainly calcareous ooze-chalk coincided with a decrease in NRM intensity of at least one order of magnitude, to $1.0 \times 10^{-8} \mathrm{G}$. Diagenesis is the probable reason for this behavior, although the dilution of magnetic carriers as the result of higher accumulation rates may also be a factor. A tectonic analysis using data from samples with stable remanence indicates a northward plate motion of about $0.3 \% / \mathrm{m} . \mathrm{y}$. during the last $18 \mathrm{~m} . y$. , a rate that agrees with most previous reconstructions of Pacific Plate motion.
\end{abstract}

\section{INTRODUCTION}

The primary objective of Leg 85 of the Deep Sea Drilling Project was to analyze sediments from holes that formed a transect of the central equatorial Pacific highproductivity belt. The study was undertaken because it was hoped that the use of a tool then relatively new, the variable-length hydraulic piston corer (VLHPC), would permit the recovery of largely undisturbed sediment from as deep as $300 \mathrm{~m}$ sub-bottom; undisturbed sediment was desired because it would permit the highest possible stratigraphic resolution of the cored material. The study of paleomagnetism was considered central to the studies to be undertaken, especially those using the pre-Pliocene sequences, for which correlation between calcareous nannofossil datums and magnetic reversals is incomplete.

The VLHPC had been used for paleomagnetic research in the Atlantic (Kent and Spariosu, 1982a, b; Tauxe et al., 1983), but it had never been used before in this part of the Pacific, although successful paleomagnetic work has been done in the central equatorial Pacific with conventional piston cores (Hays et al., 1969; Opdyke, 1972; Hammond et al., 1974; Opdyke et al., 1974; Theyer and Hammond, 1974; Theyer et al., 1978). The rotary-drilled cores acquired during four previous DSDP cruises to the

\footnotetext{
${ }^{1}$ Mayer, L., Theyer, F., et al., Init. Repts. DSDP, 85: Washington (U.S. Govt. Printing Office). Contribution No. 1463 of the Hawaii Institute of Geophysics.

2 Addresses: (Weinreich) Institut für Geophysik, Ruhr-Universitat Bochum, 4630 Bochum, Federal Republic of Germany; (Theyer, present address) Department of Geological Sciences, University of Southern California, Los Angeles, CA 90089.
}

central Pacific have yielded meager paleomagnetic results. It has generally been thought that drilling disturbance has been responsible for the poor results, although workers also observed that the older, highly calcareous oozes yielded very weak intensities. By using the VLHPC we hoped to establish magnetostratigraphic reference profiles for the entire section, including the Neogene, for this region. Unfortunately, our aims were thwarted, not by drilling disturbance or operational difficulties but by the nature of the magnetic remanence of the older sediments.

\section{MATERIALS AND METHODS}

Square, plastic boxes with a capacity of approximately $7 \mathrm{~cm}^{3}$ were used to sample the working half of each core. We wanted to achieve a stratigraphic resolution of about 0.05 to $0.1 \mathrm{~m} . \mathrm{y}$. at all levels. Therefore, except where the sediment was disturbed, we acquired samples at roughly equal time intervals, using shipboard-derived rates of sedimentation to adjust the sampling intervals. Where possible we also preferentially sampled horizons that showed horizontal bedding in order to avoid parts of the section that might be affected by subtle coringinduced disturbance. The directions and intensities of $\mathrm{NRM}_{0}$ (untreated natural remanent magnetization) were measured on board on selected samples by using a Digico spinner magnetometer; the results of this preliminary work are reported in the site chapters (this volume). For the present study, samples from about upper Miocene to Recent were analyzed at the Ruhr-Universität Bochum 
(RUB), and samples older than about upper Miocene were analyzed at the Hawaii Institute of Geophysics (HIG). All except a few measurements were made by using cryogenic magnetometers (Goree and Fuller, 1976). Alternating-field (AF) demagnetization studies were performed at RUB by using a Schonstedt GSD-1 single-axis demagnetizer and at HIG by using a three-axis sample tumbler, which was also driven by a GSD-1. For the sediments measured at RUB (upper Miocene and younger), systematic stepwise AF demagnetization series were carried out on each sample in addition to the $\mathrm{NRM}_{0}$ measurement. All these series were taken to the median destructive field (MDF), and most were taken to the $500-O e$ level. The direction of the stable remanent magnetization (RM) was then determined for each sample by analyzing its demagnetization behavior. For this purpose plots of the normalized intensity of magnetization $\left(J / J_{\text {NRM }}\right)$ versus the applied demagnetization field, vector diagrams (Zijderveld, 1967), and stereographic projections of total vector and of difference vectors of magnetization (Hoffman and Day, 1978) were studied. The stable RM direction for each sample was then determined by finding the mean of at least three consecutive demagnetization steps which showed the least directional dispersion during Fisherian (Fisher, 1953) analysis (Appendix A). We interpret this stable RM direction as the magnetic component that carries the Earth's paleofield information.

Because the samples from the older sediments (those studied at HIG) were generally characterized by uniformity and extremely low intensities, they were treated in a different way. $\mathrm{NRM}_{0}$ was measured for only some of the holes and for only some depth intervals, and progressive AF demagnetization was performed solely on selected samples. After we reviewed the demagnetization series, we demagnetized all the HIG samples by applying an alternating field of $50 \mathrm{Oe}$, a level we hoped would leave measurable magnetization yet remove some viscous components. The resulting data are listed in Appendix B.

All the Leg 85 sites lie near the equator. Therefore, most of the inclination data scattered within a few degrees of zero, and $180^{\circ}$ variations in the declination data were used to define polarity changes. This made the determination of the true polarity of the samples difficult, especially since the azimuthal orientation was unknown. At first we hoped that the Kuster single-shot orientation device, which we used during VLHPC operation, would provide azimuthal orientation. This device takes a photograph, just before the VLHPC barrel strokes out, of the position with respect to a compass needle of a double line marked on the core liner. The camera is triggered as the corer falls through a nonmagnetic collar in the drill string, an arrangement that makes the exact chronological relationship between the coring and the orientation photograph uncertain. Many mechanical, photographic, and handling difficulties were encountered with the Kuster tool, but about $57 \%$ of the slides recorded data. Unfortunately, onshore paleomagnetic analysis made it obvious that there are systematic rotations in the declination data that wholly negate the device's usefulness for azimuthal orientation purposes. For ex- ample, the downhole declination data for Cores 4 and 5 of Hole 573 show continuous clockwise and anticlockwise rotations of different magnitudes in addition to the $180^{\circ}$ polarity offsets (Fig. 1). In Core 4 there is an anticlockwise rotation of approximately $120^{\circ}$; in Core 5 there is a clockwise rotation of about $200^{\circ}$; and neither rotation is constant. These variations are due to relative motion between the sediment, core liner, and possibly even the VLHPC barrel itself. Therefore, only the uppermost part of a VLHPC core may be oriented as shown by the Kuster photo, and even that is open to question, because neither the vertical position of the camera nor the exact time at which the camera takes the photo is known (motion between sediment, core liner,

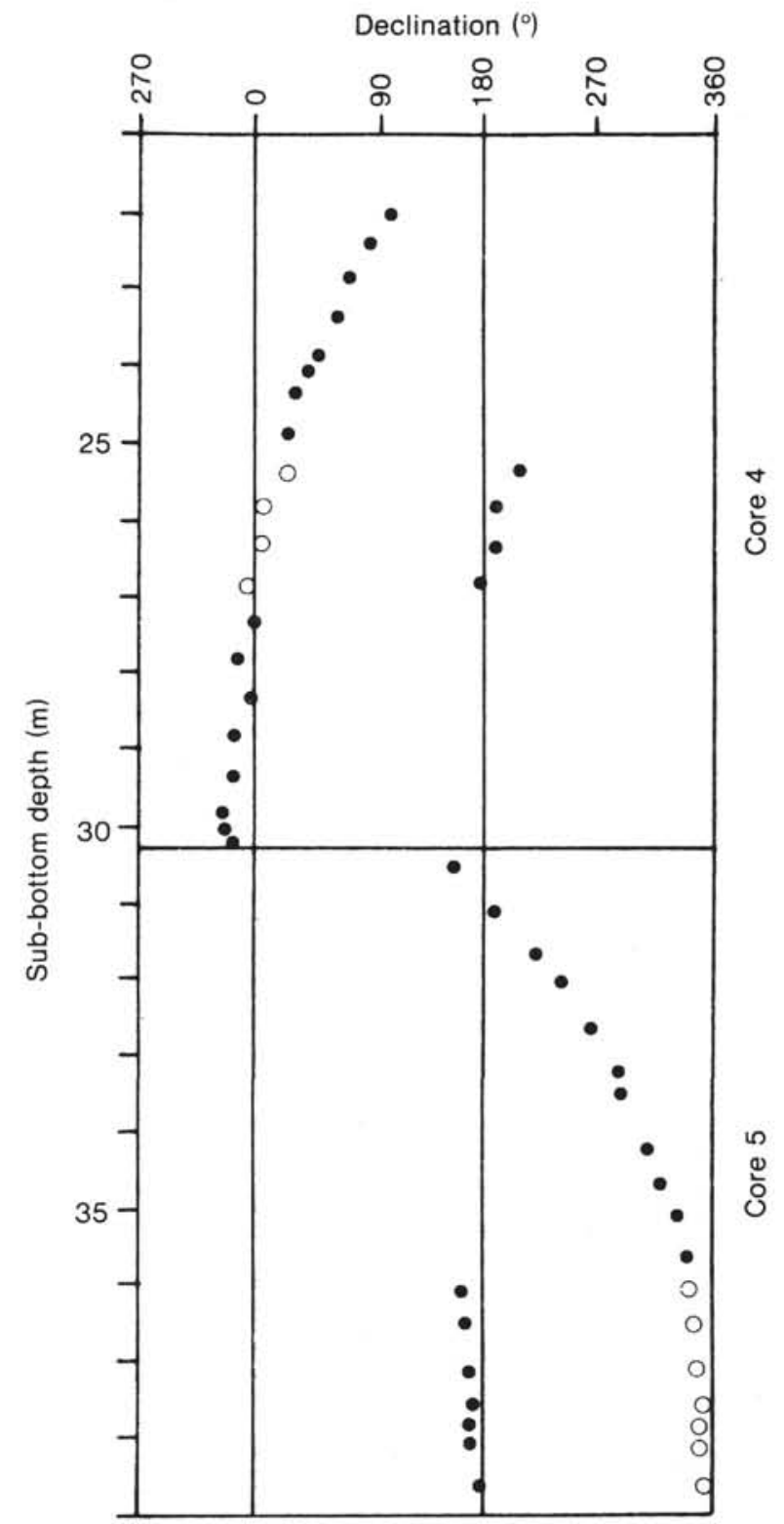

Figure 1. Downcore variation of the uncorrected declination data for Cores 4 and 5 of Hole 573, showing rotation produced during VLHPC coring. See text for details. Solid circles indicate actual data; open circles are $180^{\circ}$ projections made to provide visual continuity in the trends. 
and barrel, may occur between the time when the photograph is exposed and the actual coring process). In other words, the photo shows the azimuthal orientation of the core liner at a certain instant, but the azimuthal orientation may change before or as the coring actually takes place. In this report, therefore, only relative declination directions are used (those found by referring to the position of the line marked on the core liner).

The younger cores (those measured at RUB) yielded acceptable magnetic data, and the declination data are mostly well grouped azimuthally $\left(180^{\circ}\right.$ shifts indicate polarity changes). Thus, average declinations were calculated for these cores as described by Kent and Spariosu (1982a). We did not do this for the sediments analyzed at $\mathrm{HIG}$, because these older sediments displayed weak NRM intensities and were consequently nearly useless for magnetostratigraphy. Arbitrary declinations are shown from core to core for these sediments. All data, corrected and uncorrected, are listed in Appendixes A and B.

The data in Appendixes A and B provide the basis for our magnetostratigraphic and tectonic interpretations. The polarity sequences were constructed by assuming that the topmost interval of continuous polarity is the Brunhes Normal Chron and that the first $180^{\circ}$ shift of declination is the Brunhes/Matuyama boundary at $0.72 \mathrm{Ma}$ (Ness et al., 1980). The underlying cores were interpreted by assuming that $180^{\circ}$ changes between two cores resulted not from changes in polarity but rather from the rotation of the cores themselves. The azimuthal average of the uppermost (Brunhes) declination sequence was then arbitrarily set at $0^{\circ}$, which resulted in an azimuthal average of $180^{\circ}$ for the reversed Matuyama sequence that followed below. Subsequent downcore $180^{\circ}$ swings of declination were then used to define the magnetostratigraphy. In some intervals this method failed, and the interpretation of polarity was guided by inclination behavior and biostratigraphy. Finally, a few intervals were so difficult to interpret that the sequence of polarities can only be considered an estimate.

\section{RESULTS AND DISCUSSION}

\section{Magnetic Behavior of Sediments}

The direction of the stable RM may be overprinted by weaker magnetic components of low coercivity (Kent and Lowrie, 1974). Figure 2 illustrates a sample from a cyclic siliceous-calcareous lithologic unit in Hole 573A (Core 2) that carries multiple components of magnetization with almost antiparallel directions. The components are revealed by the increase in the normalized magnetization from an $\mathrm{NRM}_{0}$ level of $5.8 \times 10^{-6} \mathrm{G}$ (Fig. 2A) and by the wide scatter in the total vector of magnetization in the stereographic projection (Fig. 2C). After about 200 Oe these weaker components are removed, and the very high MDF of $652 \mathrm{Oe}$ indicates the high stability of the stable RM (Fig. 2A). The vector diagram (Fig. 2B) and the difference-vector projection (Fig. 2D) show the multiple components of the system in more detail. One weaker component is removed at about $50 \mathrm{Oe}$ and another at $200 \mathrm{Oe}$. This behavior is reflected in both the xy- and the yz-planes (Fig. 2B). The ensuing monotonous de- crease of the orthogonal components toward zero suggests that there are no other components. The weak component of the sample of Figure 2 can be identified as a viscous magnetization acquired during the Brunhes Chron, whereas the stable component reflects the reversed direction of the Matuyama Chron.

Figure 3 shows the response of Sample 574C-34-2, $109-111 \mathrm{~cm}$, which is from a metalliferous sediment layer overlying basalt. The sample is characterized by a relatively high $\mathrm{NRM}_{0}$ intensity $\left(7.4 \times 10^{-6} \mathrm{G}\right)$. The increase in the normalized intensity curve (Fig. 3A) and the vector diagram (Fig. 3B) suggest that it too may contain separate components of magnetization. The weaker component persists to about $100 \mathrm{Oe}$, and the MDF of the components with higher stability lies at $310 \mathrm{Oe}$. Even though the stereographic projection of the total vector of magnetization shows only one direction (Fig. 3C), the difference-vector diagram (Fig. 3D) allows a qualitative and quantitative definition of the two antiparallel directions of the sample. The inclination data define the polarity of the low-coercivity component ( 0 to $100 \mathrm{Oe})$ as reversed and that of the stable component as normal (Fig. 3D).

Sample 573A-1-3, 90-92 cm, which is from a siliceouscalcareous lithologic unit of Brunhes age, carries an $\mathrm{NRM}_{0}$ intensity of $2.5 \times 10^{-6} \mathrm{G}$. Its behavior (Fig. 4) suggests a single component. The normalized intensity shows a continuous decrease during stepwise AF cleaning (Fig. 4A). The vector diagram (Fig. 4B) and the stereographic projections (Figs. 4C, D) show a single pattern, and the MDF of 270 Oe reflects the inherent stability of the component.

Finally, Figure 5 gives an example of useless data obtained on a weakly magnetized sample. This material (Sample 575A-18-1, 144-146 cm) is from highly calcareous ooze and had an $\mathrm{NRM}_{0}$ intensity of $6.7 \times 10^{-7} \mathrm{G}$. Although the stereographic projection in Figure 5C suggests stability, the other diagrams (Figs. 5A, B, D) reveal its erratic behavior upon AF treatment. This sample was typical of the highly calcareous (older than uppermost Miocene) sediments recovered during Leg 85 .

\section{Leg 85 Magnetostratigraphy}

A satisfactory magnetostratigraphy was derived for the Pliocene and Pleistocene sediments, which had intensities that were in a reasonable range $\left(>10^{-8} \mathrm{G}\right)$ for measurement on the cryogenic magnetometer, even after AF demagnetization. In contrast, the calcareous oozes from the Miocene and below allowed the determination of magnetostratigraphic sequences at only a few intervals. The boundary between usable and unusable data coincided with the boundary between cyclic siliceouscalcareous ooze and calcareous ooze. Within the lower, highly calcareous sediments, the weakness of the $\mathrm{NRM}_{0}$ intensities made AF demagnetization impossible, and the $\mathrm{NRM}_{0}$ data alone did not contain coherent stratigraphies.

Polarity boundaries had to be inferred from $180^{\circ}$ shifts of declination because of the shallowness of the inclinations. However, some of the sites crossed the equator, and in the absence of azimuthal orientation neither declination nor inclination was an unequivocal guide to po- 

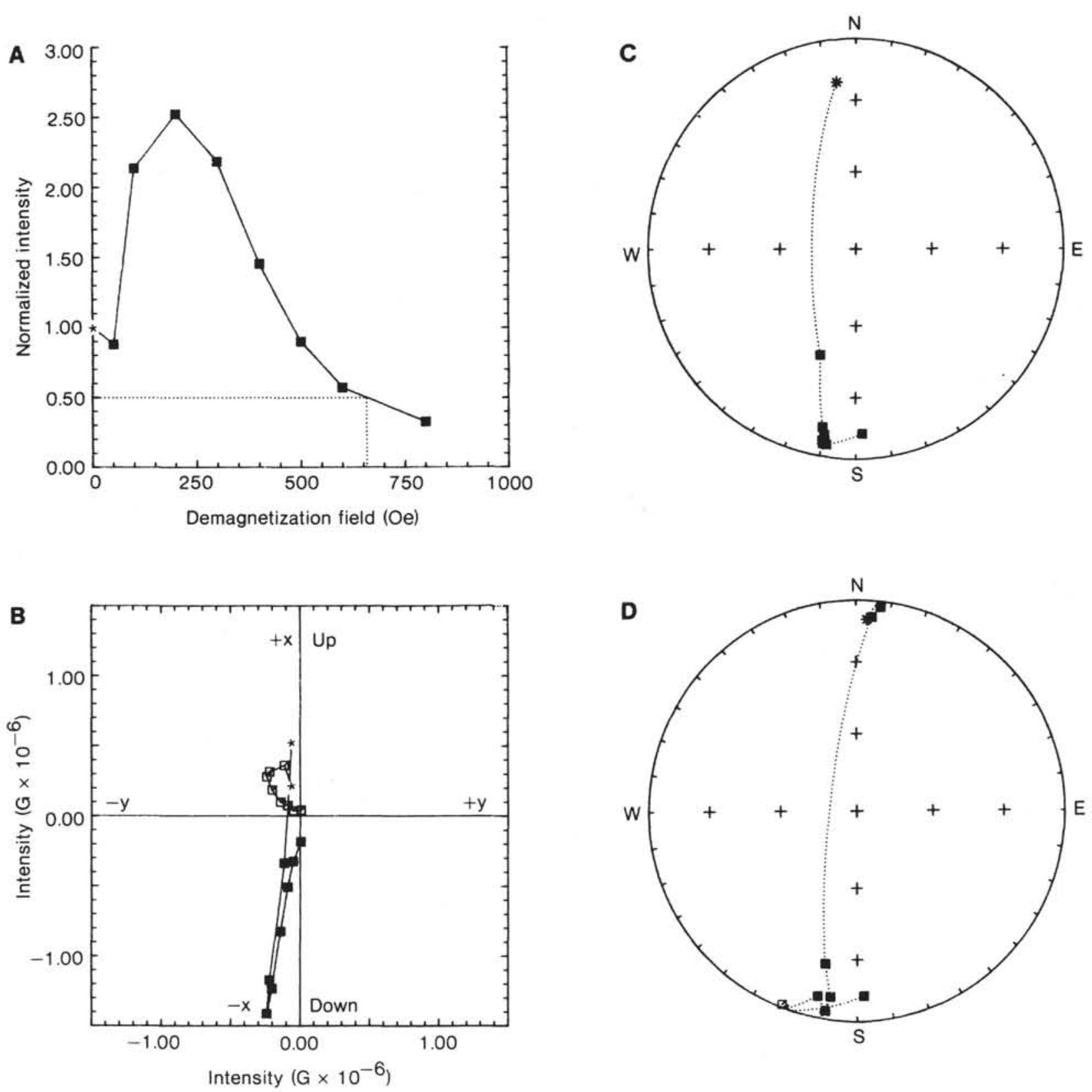

Figure 2. Behavior of the magnetization of Sample 573A-2-3, 67-69 cm, taken from a cyclic siliceous-calcareous lithologic unit, during successive AF demagnetization. A. Normalized intensity of magnetization plotted against the applied demagnetization field. Dotted line indicates the MDF. Asterisk indicates $\mathrm{NRM}_{0}$. B. Vector diagram of the orthogonal components $(\mathrm{x}, \mathrm{y}, \mathrm{z})$ of magnetization. Closed symbols represent the horizontal $(x, y)$ plane, open symbols the vertical $(z, y)$ plane. Asterisk indicates $\mathrm{NRM}_{0}$ components. C. Stereographic projection of the total vector of magnetization. Closed symbols indicate normal, open reversed polarity. Asterisk indicates $\mathrm{NRM}_{0}$ direction. All directions are connected by great-circles. D. Stereographic projection of the difference vectors. Closed symbols indicate normal, open symbols reversed polarity. The starting point is marked by an asterisk.

larity. We developed our magnetostratigraphy for these sites by (1) comparing the relative lengths of the polarity intervals with the known polarity sequence and (2) referring to biostratigraphy. In the metalliferous sediment layer at Site 574 (which contains the Eocene/Oligocene boundary), intensities increased to about $10^{-5} \mathrm{G}$, but despite the apparent stability of most of the samples from this layer (see the AF demagnetization series in Fig. 3), the directions (especially the inclinations) fail to indicate a coherent stratigraphic pattern.

\section{Site 571}

Only one core $(7.10 \mathrm{~m})$ was recovered at Site 571 . Shipboard paleomagnetic analysis of $\mathrm{NRM}_{0}$ indicates that the polarity pattern is coherent throughout this core. Biostratigraphic dating defined the core as Quaternary, from Recent to about $0.46 \mathrm{Ma}$. Thus, it correlates with the Brunhes Chron. $\mathrm{NRM}_{0}$ intensities vary from $2.4 \times$ $10^{-6} \mathrm{G}$ to $7.4 \times 10^{-6} \mathrm{G}$, with a geometric mean of 4.7 $\times 10^{-6} \mathrm{G}$. The AF-cleaned data, especially the inclina- 

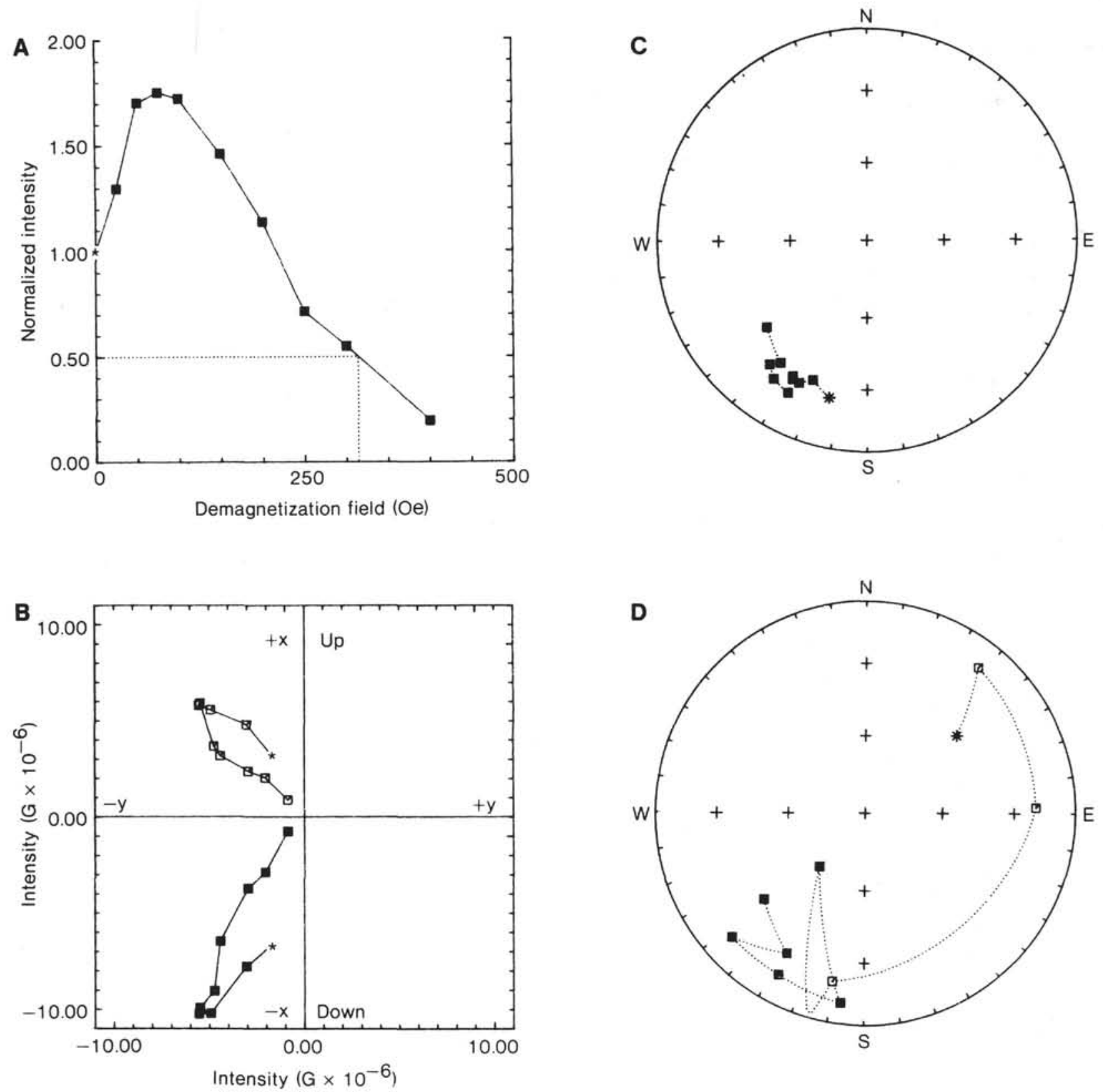

Figure 3. Behavior of Sample 574C-34-2, 109-111 cm. This sample was rotary drilled from the metalliferous sediments overlying basement at Site 574. See Figure 2 caption for details.

tions, show very stable behavior; we were able to derive a mean stable inclination of $7.6^{\circ}$, which gives a dipole paleolatitude of $3.8^{\circ} \mathrm{N}$. This is in good agreement with the present site latitude of $4.0^{\circ} \mathrm{N}$.

Site 572

No magnetostratigraphy could be established for Site 572. Most of the $\mathrm{NRM}_{0}$ intensities of the sediments recovered with the HPC were at the noise level of the cryogenic magnetometer, so that demagnetization studies were pointless. In addition, the $\mathrm{NRM}_{0}$ directional data show almost random scatter. Although some intervals in the older, rotary-drilled sediments show high intensities, their inclinations are scattered about zero, and AF cleaning did not improve the results. The data are therefore presented in tabular form only (Appendix B).

\section{Site 573}

Site 573 is the southernmost site in a three-site northsouth transect along about $133^{\circ} \mathrm{W}$. The transect cuts across the north-central region of the equatorial highproductivity zone. Two holes were cored with the VLHPC at Site 573, and one was rotary drilled to basement (which was at a sub-bottom depth of $529 \mathrm{~m}$; Table 1). Both the sediment (which ranged in age from Quaternary to upper Eocene) and the basalt were sampled for paleomagnetic analysis. A drastic decrease in intensity occurs at about $45 \mathrm{~m}$ sub-bottom in both Holes 573 and 573A 

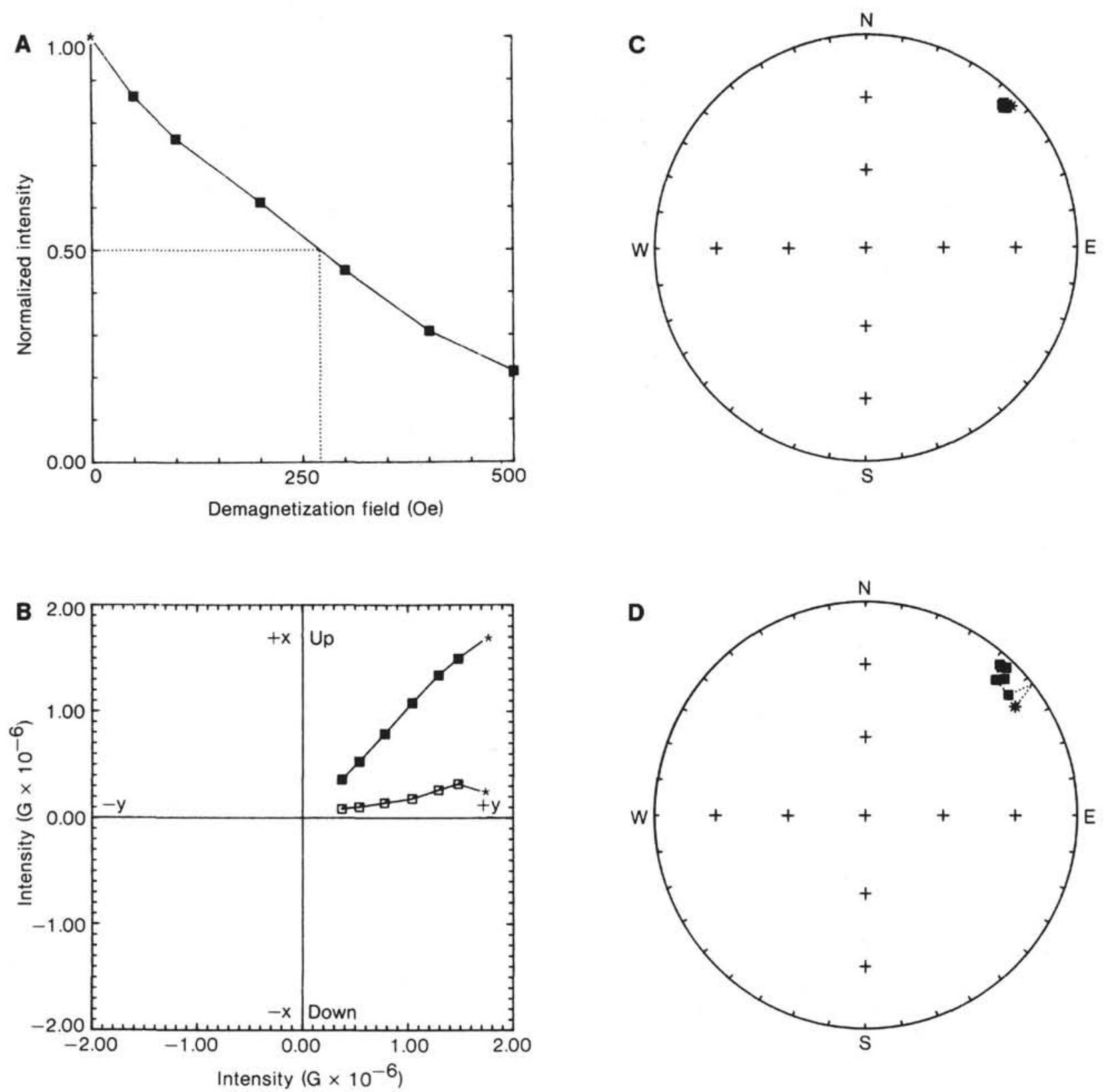

Figure 4. Behavior of Sample 573A-1-3, 90-92 cm. This sample was taken from Brunhes-age siliceous-calcareous sediments. See Figure 2 caption for details.

(Fig. 6); it defines the lower limit of the interpretable data and coincides with a change in lithology from cyclic siliceous-calcareous ooze (Unit I) to calcareous ooze (Unit II). The decrease in intensity exceeds one order of magnitude. The intensities throughout lithologic Unit I show a variation about a mean of $2.31 \pm 1.74 \times 10^{-6}$ $\mathrm{G}$, a mean similar to that at Site 571 . The intensities exhibited by other Neogene siliceous-calcareous pelagic sediments are comparable (Kent and Spariosu, 1982a, b; Tauxe et al., 1983). Throughout the underlying lithostratigraphic Unit II (from $45.1 \mathrm{~m}$ to $520.0 \mathrm{~m}$ sub-bottom), there are intervals of remarkably constant intensities that average about $10^{-7} \mathrm{G}$, but these intervals do not provide interpretable data. Within the metalliferous chalk to claystone unit (lithologic Unit III, from $520.0 \mathrm{~m}$ to $526.6 \mathrm{~m}$ sub-bottom) and the short upper Eocene sequence of pelagic limestone (lithologic Unit IV, $526.6 \mathrm{~m}$ to $529.0 \mathrm{~m}$ sub-bottom), the intensities increase to a maximum of about $10^{-3} \mathrm{G}$, but the data are useless for magnetostratigraphic work because of their almost random scatter. The inclinations, which during the latest Eocene and early Oligocene are expected to reflect a southerly latitude of some 7 to $10^{\circ}$, do not allow the identification of polarity sequences. The $\mathrm{NRM}_{0}$ intensities of the six basalt samples measured have a mean of $3.7 \pm 2.7 \times 10^{-4} \mathrm{G}$, which is typical of such material (Petersen and Roggenthen, 1980).

The magnetostratigraphies developed for the uppermost parts of Holes 573 and 573A are shown in Figure 6. Core-to-core interpretation. was straightforward because 

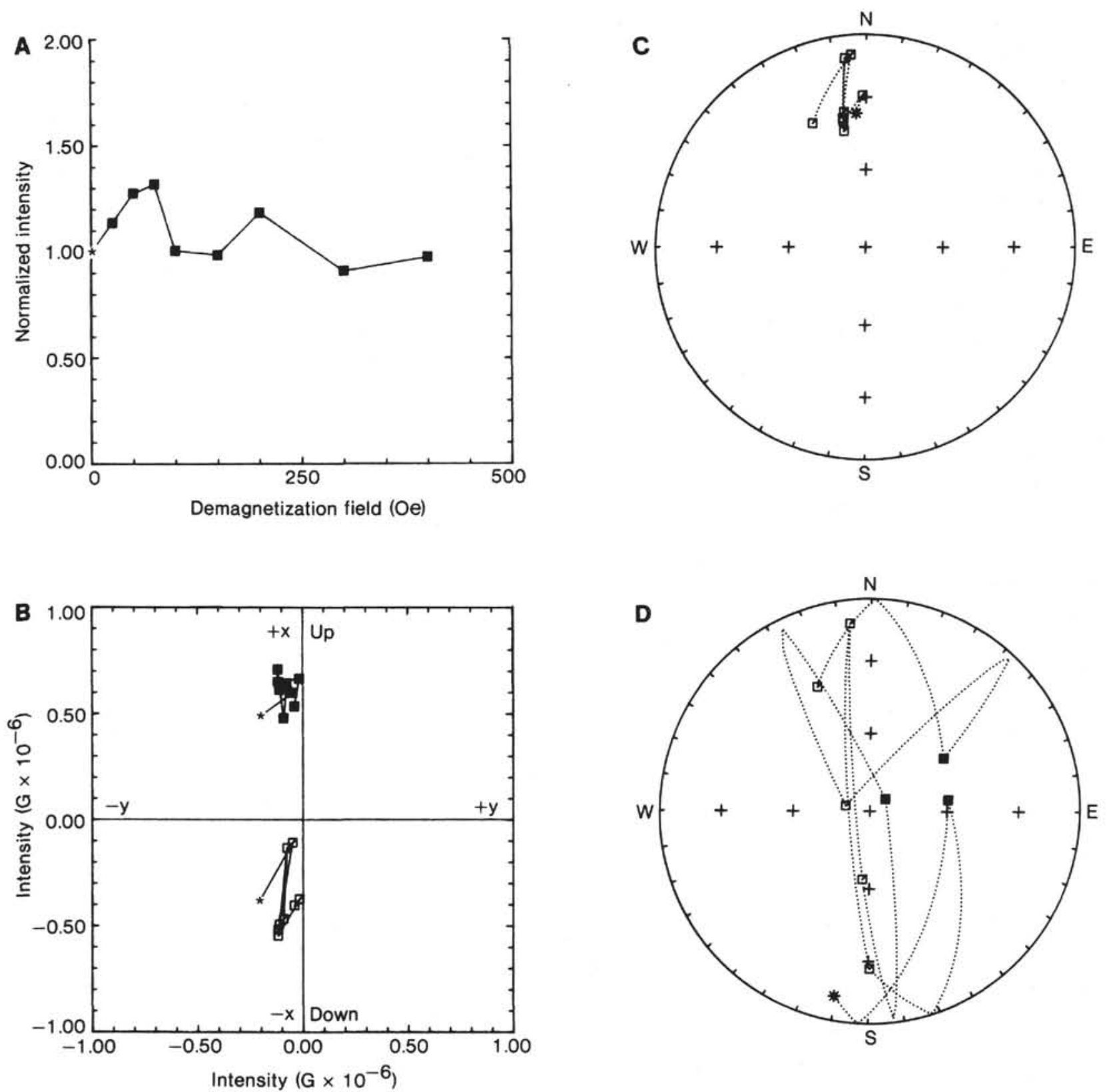

Figure 5. Behavior of Sample 575A-18-1, 144-146 cm. This sample represents the majority of sediments encountered on Leg 85 . It comes from a highly calcareous unit and is very weakly magnetized, its NRM intensity being $6.7 \times 10^{-7} \mathrm{G}$. See Figure 2 caption for details.

of the $180^{\circ}$ declination shifts in Hole 573 . In both holes the record spans from the lower Mammoth transition at 3.19 Ma (Ness et al., 1980) to the Brunhes/Matuyama boundary. Table 2 lists the depths of polarity changes found in both holes; it also gives the position of the last sample of the upper polarity sequence and the first sample of the lower sequence, thus defining the depth-uncertainty range for each polarity change (Fig. 7, dotted intervals). As shown in Table 2 and Figure 7, the stratigraphically equivalent $180^{\circ}$ shifts in declination occur at different depths in the holes. In Figure 7, the points that define the same polarity transition in the holes plot along three $45^{\circ}$ line segments, and the top segment is offset from zero. Within the depth intervals indicated by each $45^{\circ}$ line segment, the drilling-related displacement of material is absent and the net sedimentation rate is constant. The breaks between the line segments may be explained in three ways: (1) net sedimentation rates may have changed from hole to hole, although such variation is unlikely, given the general uniformity of the region's seismic properties and sedimentation rates; (2) coring procedures may have introduced disturbances in the form of missing material and partial core recovery; and (3) shifts in the magnetic boundaries may have been introduced by reworking, direct disturbance, or postdepositional effects (L $\varnothing$ vlie, 1976).

The top line segment may be offset from zero because the top of the section in Hole 573A is missing. However, we believe that solution or erosion caused the sediment sequence from the Brunhes Chron in Hole 573A to be 
A
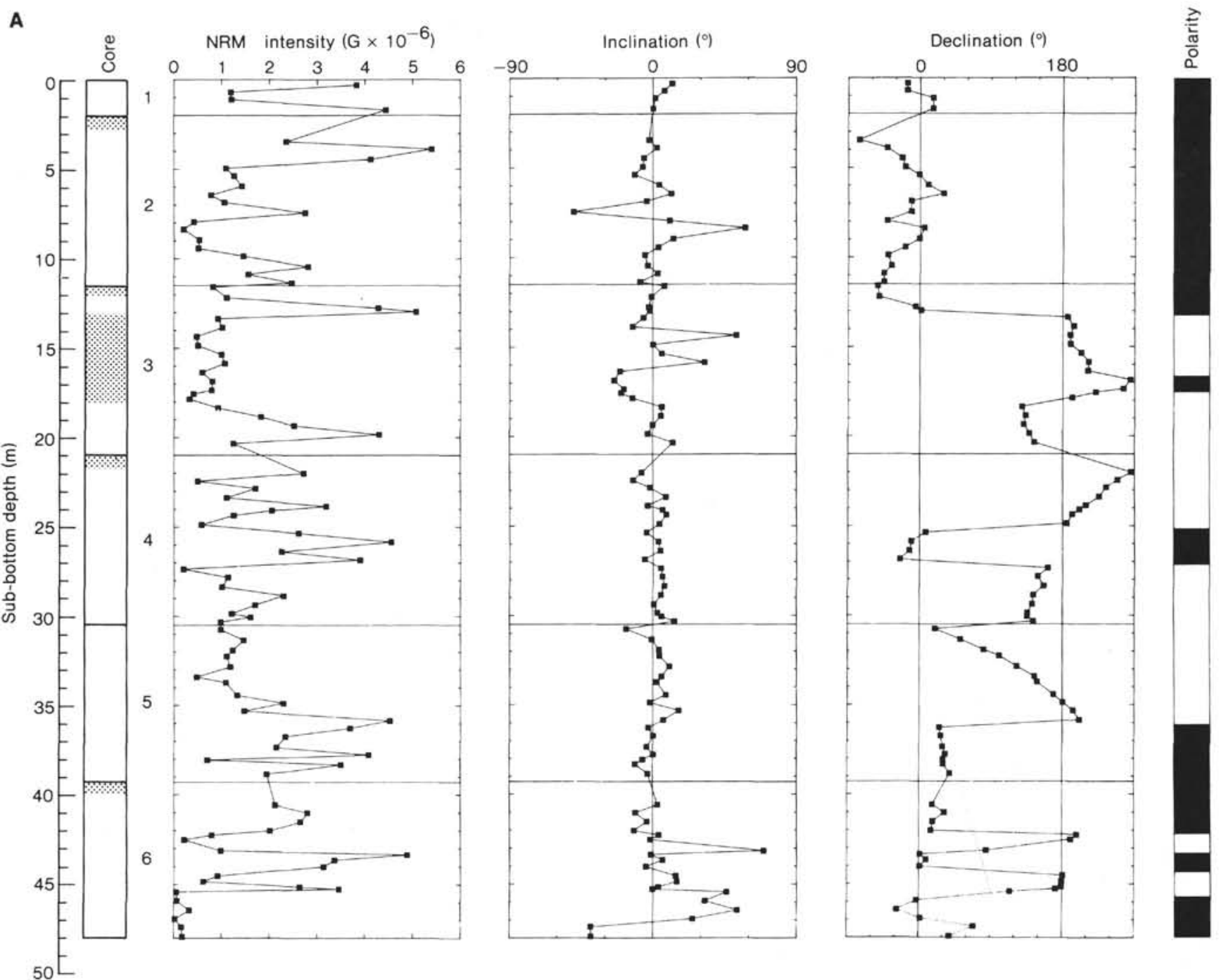

Figure 6. NRM intensity and paleomagnetic polarity for (A) Hole 573 and (B) Hole 573A. In the column showing the depths of the cores, dotted intervals are those identified on the barrel sheets as disturbed by drilling. The remaining columns show the downhole variation of NRM intensity; stable inclination and corrected declination (see text for details); and paleomagnetic polarity (derived from $180^{\circ}$ shifts in the declination data). Black indicates intervals of normal polarity, white intervals of reversed polarity, and striping intervals that could not be interpreted.

Table 1. Summary of Leg 85 coring.

\begin{tabular}{|c|c|c|c|c|c|c|}
\hline Site & Location & $\begin{array}{l}\text { Maximum } \\
\text { depth of } \\
\text { penetration } \\
\text { (m) }\end{array}$ & Hole & $\begin{array}{c}\text { Interval } \\
\text { cored (m } \\
\text { sub-bottom) }\end{array}$ & $\begin{array}{l}\text { Estimated } \\
\text { age of } \\
\text { oldest } \\
\text { sediment } \\
\text { (Ma) }\end{array}$ & $\begin{array}{c}\text { Nature } \\
\text { of } \\
\text { basement }\end{array}$ \\
\hline 571 & $\begin{array}{r}114^{\circ} 08.53^{\prime} \mathrm{W} \\
3^{\circ} 59.84^{\prime} \mathrm{N}\end{array}$ & 7.1 & 571 & $0-7.1$ & 0.46 & Not reached \\
\hline 572 & $\begin{array}{l}113^{\circ} 50.52^{\prime} \mathrm{W} \\
1^{\circ} 26.09^{\prime} \mathrm{N}\end{array}$ & 486.0 & $\begin{array}{l}572 \\
572 \mathrm{~A} \\
572 \mathrm{~B} \\
572 \mathrm{C} \\
572 \mathrm{D}^{\mathrm{a}}\end{array}$ & $\begin{array}{r}0-19.0 \\
0-154.0 \\
154.0-172.1 \\
0-169.5 \\
151.0-486.0\end{array}$ & $14-15$ & Basalt \\
\hline 573 & $\begin{array}{l}133^{\circ} 18.57^{\prime} \mathrm{W}, \\
0^{\circ} 29.91^{\prime} \mathrm{N}\end{array}$ & 529.0 & $\begin{array}{l}573 \\
573 \mathrm{~A} \\
573 \mathrm{~B}^{\mathrm{a}}\end{array}$ & $\begin{array}{c}0-158.6 \\
0-53.2 \\
138.5-529.0\end{array}$ & $38-39$ & Basalt \\
\hline 574 & $\begin{array}{c}133^{\circ} 19.81^{\prime} \mathrm{W} \\
4^{\circ} 12.52^{\prime} \mathrm{N}\end{array}$ & 532.5 & $\begin{array}{l}574 \\
574 \mathrm{~A} \\
574 \mathrm{~B}^{\mathrm{a}} \\
574 \mathrm{C}^{\mathrm{a}}\end{array}$ & $\begin{array}{r}0-206.5 \\
0-180.2 \\
185.0-204.0 \\
194.5-532.5\end{array}$ & $38-39$ & Basait \\
\hline 575 & $\begin{array}{c}135^{\circ} 02.16^{\prime} \mathrm{W} \\
5^{\circ} 51.00^{\prime} \mathrm{N}\end{array}$ & 208.3 & $\begin{array}{l}575 \\
575 \mathrm{~A} \\
575 \mathrm{~B} \\
575 \mathrm{C}\end{array}$ & $\begin{array}{c}0-98.6 \\
0-208.3 \\
3.3-119.0 \\
0-15.8\end{array}$ & 22 & Not reached \\
\hline
\end{tabular}

${ }^{a}$ Rotary drilled. more compressed than in Hole 573, an interpretation consistent with the shortness of both the first reversed interval of the Matuyama Chron and the Jaramillo subchron in Hole 573A. On the other hand, both the Jaramillo subchron and the Brunhes/Matuyama boundary in Hole 573 fall within an interval disturbed by coring (Fig. 6A).

The first break in the $45^{\circ}$ line (Fig. 7) occurs in the early part of the Matuyama Chron. It too can be interpreted as a compaction effect, because again there is some shortening in Hole 573A, but in this case it is more likely to be due to the coring process. The Jaramillo, Olduvai, and all the polarity changes in the Gauss Chron follow the predicted sequence. Between the Matuyama/Gauss boundary and the beginning of the Olduvai subchron, there is a second break in the $45^{\circ}$ line (Fig. 7); it occurs at the boundary between Cores 4 and 5 in Hole 573 and between Cores 3 and 4 in Hole 573A. It is likely that sediments were lost between those cores: 
B
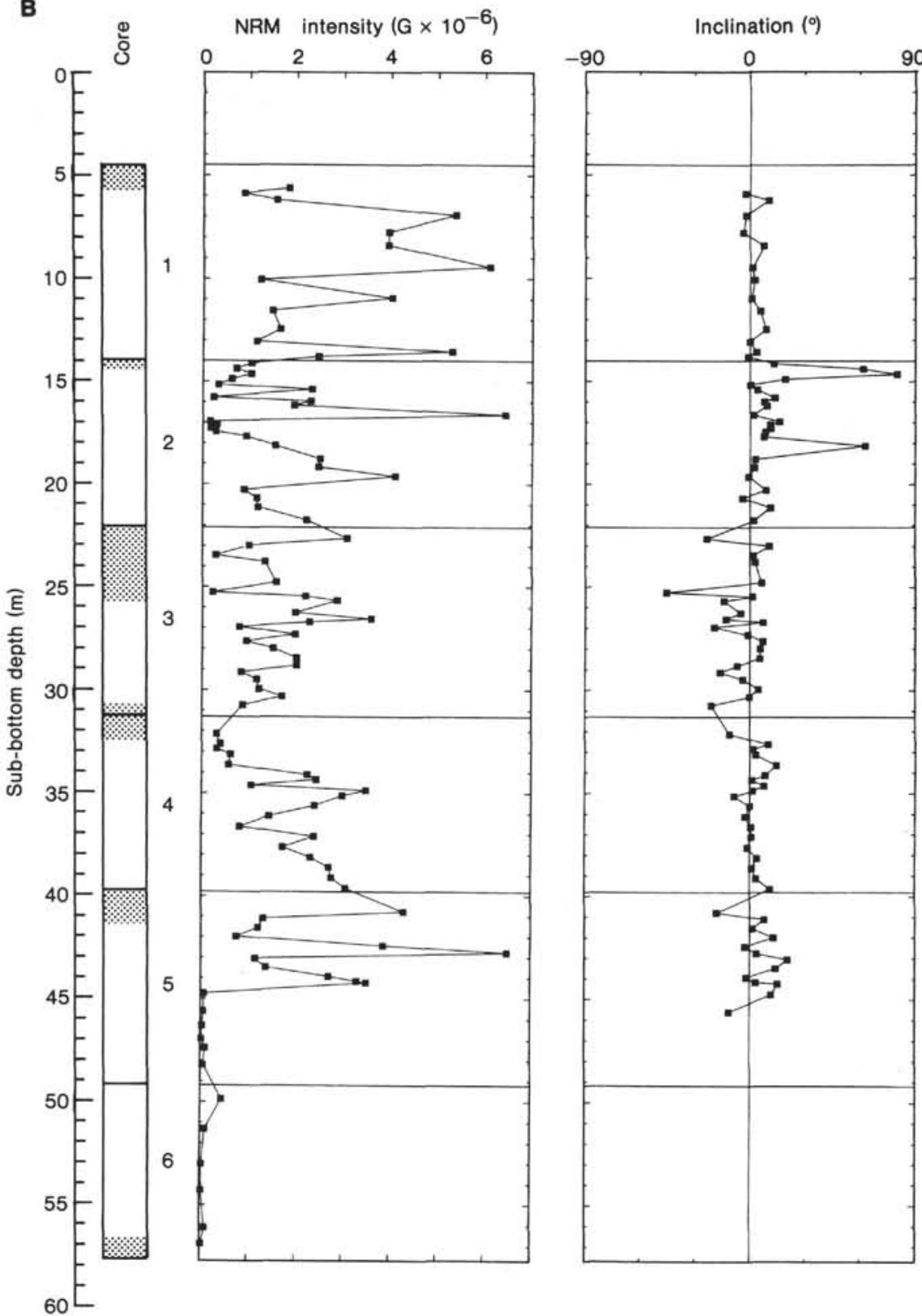

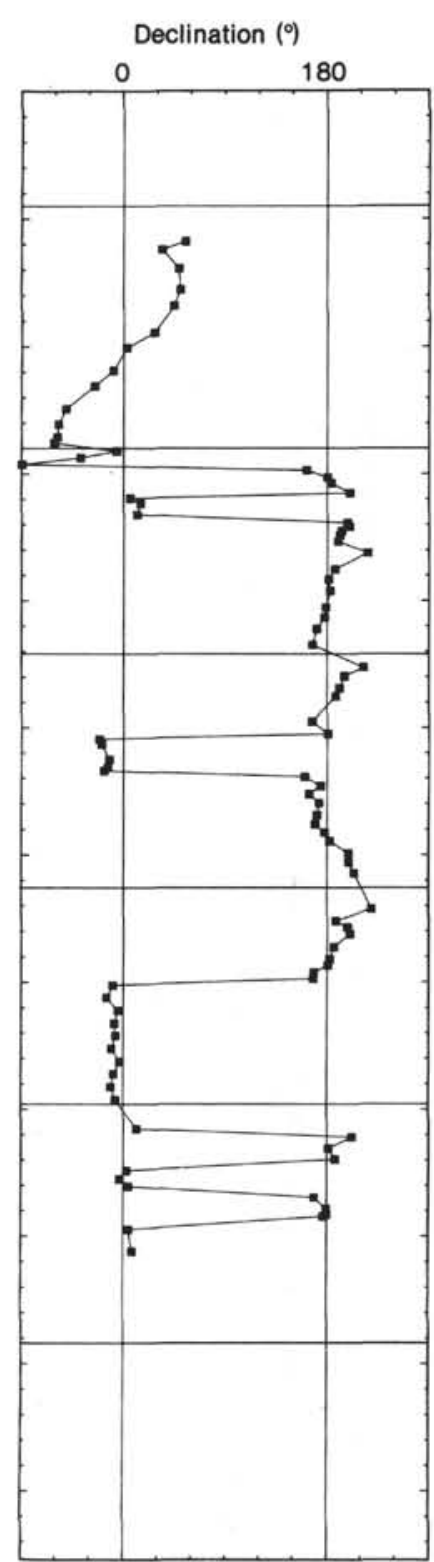

춯

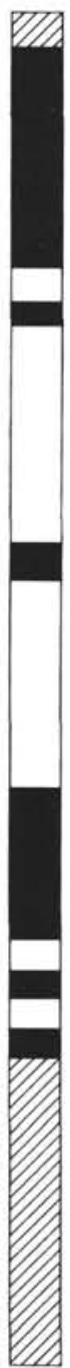

Figure 6. (Continued),

adding about $1 \mathrm{~m}$ of sediment between Cores 4 and 5 in Hole 573 and between Cores 3 and 4 in Hole 573A would produce a straight line from the Gauss/Gilbert boundary to the Jaramillo subchron.

\section{Site 574}

Site 574 , at $4^{\circ} 12.52^{\prime} \mathrm{N}$ and $133^{\circ} 19.81^{\prime} \mathrm{W}$, was near the northern edge of the high-productivity belt. A sediment sequence from upper Eocene to Quaternary was recovered, as well as a few pieces of basalt drilled from basement. All cores were sampled (Appendixes A and B), although only the samples from the uppermost 10 cores of Holes 574 and 574A are discussed here because of the low intensities lower in the section. The intensities are characteristic of lithologic Unit I (cyclic siliceous- calcareous ooze) down to about $84 \mathrm{~m}$ sub-bottom; they show significant variations that are in accord with the lithostratigraphic subunits (site chapters, this volume). For Subunit IA (Hole 574), a mean intensity of $3.93 \pm$ $2.94 \times 10^{-6} \mathrm{G}$ was obtained. In both holes there is a very sharp decrease in intensity of more than one order of magnitude at the transition from Subunit IA to Subunit IB. The intensities of the sediments in Subunit IC are slightly lower than in Subunit IA $(2.12 \pm 1.14 \times$ $10^{-6} \mathrm{G}$ in Hole 574 and $1.56 \pm 1.16 \times 10^{-6} \mathrm{G}$ in Hole 574A). Throughout lithologic Unit II (calcareous oozechalk), the intensities are in the range of $10^{-7} \mathrm{G}$ (Fig. 8), and the resulting data are not usable for magnetostratigraphy. With few exceptions, the magnetic directions and the intensities to $520 \mathrm{~m}$ sub-bottom are either randomly 
Table 2. Magnetostratigraphy for Holes 573 and 573A.

\begin{tabular}{|c|c|c|c|c|c|}
\hline \multirow[b]{2}{*}{ Boundary } & \multirow[b]{2}{*}{$\begin{array}{c}\mathrm{Age} \\
(\mathrm{Ma})^{\mathrm{a}}\end{array}$} & \multicolumn{2}{|c|}{ Hole 573} & \multicolumn{2}{|c|}{ Hole 573A } \\
\hline & & $\begin{array}{c}\text { Depth of } \\
\text { boundary } \\
\text { (m sub-bottom) }\end{array}$ & $\begin{array}{l}\text { Uncertainty range } \\
\text { (Core-Section, } \\
\text { level in cm) }\end{array}$ & $\begin{array}{c}\text { Depth of } \\
\text { boundary } \\
\text { (m sub-bottom) }\end{array}$ & $\begin{array}{l}\text { Uncertainty range } \\
\text { (Core-Section, } \\
\text { level in } \mathrm{cm})^{\mathrm{c}}\end{array}$ \\
\hline Brunhes/Matuyama & 0.72 & 13.17 & $3-1,147 / 3-2,36$ & 14.63 & $2-1,38 / 2-1,88$ \\
\hline upper Jaramillo & 0.91 & 16.61 & $3-4,36 / 3-4,86$ & 15.98 & $2-2,28 / 2-2,68$ \\
\hline lower Jaramillo & 0.97 & 17.47 & $3-4,136 / 3-5,7$ & 16.96 & $2-2,113 / 2-3,28$ \\
\hline upper Olduvai & 1.66 & 25.12 & $4-3,88 / 4-3,136$ & 25.48 & $3-3,173 / 3-3,58$ \\
\hline lower Olduvai & 1.87 & 27.11 & $4-4,136 / 4-5,36$ & 26.77 & $3-3,147 / 3-4,36$ \\
\hline Matuyama/Gauss & 2.47 & 35.97 & $5-4,86 / 5-4,128$ & 35.19 & $4-3,33 / 4-3,84$ \\
\hline upper Kaena & 2.91 & 42.05 & $6-2,123 / 6-2,147$ & 41.25 & $5-1,99 / 5-1,131$ \\
\hline lower Kaena & 2.98 & 43.15 & $6-3,84 / 6-3,106$ & 42.52 & $5-2,69 / 5-2,114$ \\
\hline upper Mammoth & 3.07 & 44.19 & $6-24,23 / 6-4,74$ & 43.58 & $5-3,26 / 5-3,69$ \\
\hline lower Mammoth & 3.17 & 45.58 & $6-5,13 / 6-5,62$ & 44.80 & $5-3,144 / 5-4,46$ \\
\hline
\end{tabular}

${ }^{a}$ Ness et al. (1980).

b Interpolated from coring summary (site chapter).

${ }^{\mathrm{c}}$ Last sample of upper polarity sequence/first sample of lower polarity sequence.

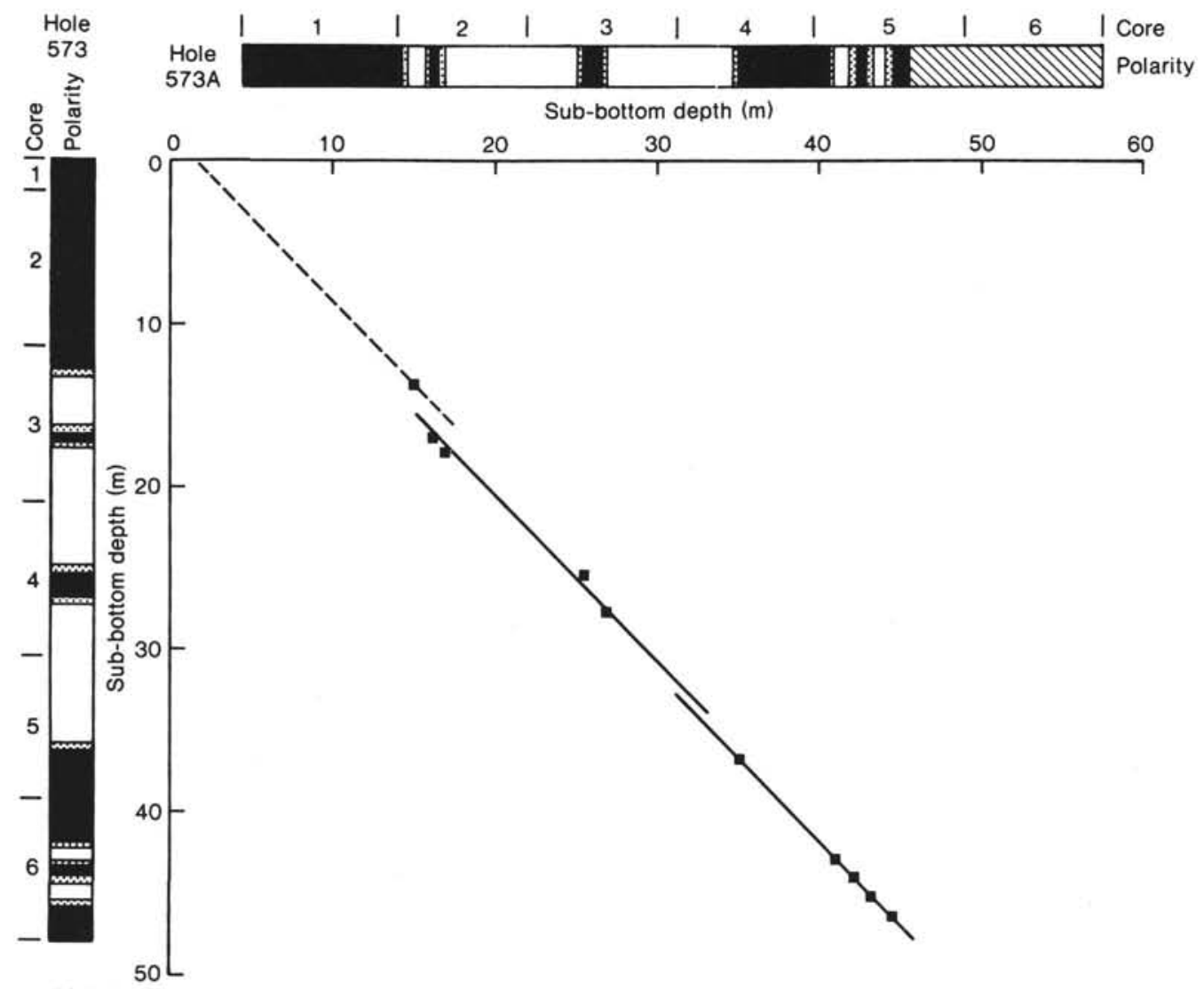

Figure 7. Comparison of paleomagnetic polarity logs for Holes 573 and $573 \mathrm{~A}$. Correlative polarity transitions are plotted. The dotted intervals above and below the transitions indicate lack of samples and thus the uncertainty in the depth of a boundary. Black indicates intervals of normal polarity, white intervals of reversed polarity, and striping intervals that were not interpreted.

scattered or remain virtually constant for considerable depth intervals, and both of these patterns preclude coherent interpretation. Typically, both the declinations and the intensities follow this pattern, possibly indicating remagnetization or chemical alteration of the magnetic carriers (Henshaw and Merrill, 1980). In the upper Eocene metalliferous chalk unit (Unit III), we measured stronger intensities (in the range of some $10^{-5} \mathrm{G}$ ), but the inclination data did not allow the establishment of a meaningful stratigraphic pattern.

\section{Hole 574}

The magnetostratigraphy for the upper 10 cores of Site 574 (Figs. 8A and B) is coherent, although it contains a gap as the result of the very low intensities of Cores 6 to 8 in lithologic Subunit IB. The polarity se- 
quence in Hole 574 starts with the Brunhes Chron in Core 1 (Fig. 8A). Core 2 contains Matuyama sediments (as defined by inclination data), and the next interval of normal polarity indicates the Olduvai subchron; the Brunhes/Matuyama boundary and the Jaramillo subchron are missing in this hole. The normal section at the bottom of Core 2 marks the top of the Gauss Chron. Throughout the upper part of Core 3, coring disturbance prohibits interpretation; the Gauss/Gilbert boundary alone is well defined. The first normal subchron within the Gilbert Chron, the Cochiti subchron, is poorly expressed in Core 4, but the lower three Gilbert subchrons can easily be identified (Fig. 8A). The transition (reversed to normal) in Core 5 defines the Gilbert/Chron 5 boundary, and the Chron 5/Chron 6 boundary can be distinguished in Core 6 . Farther below, a brief normal subchron may occur, but it is not clearly indicated because of the low intensity and the noise in the inclination data. From Section 574-6-4 to Section 574-9-4 no stratigraphy is discernible. The polarity sequence in Cores 9 and 10 indicates the presence of the youngest normal subchron in Chron 8 and the transition from Chron 8 to Chron 9. This interpretation disagrees, however, with the biostratigraphic dating of this depth interval and may well be erroneous: the polarity sequence is discontinuous with that above Core 9, and the inclination data give no indication of polarity. Our interpretation is based on a linear extrapolation and on a comparison to the length of the polarity intervals of the actual time scale (Ness et al., 1980).

\section{Hole 574 A}

Hole 574A (Fig. 8B) is similar to Hole 574 (Fig. 8A). No paleomagnetic data are available from Core 1 because of poor recovery, but Core 2 correlates with the Matuyama Chron, and the first interval of normal polarity is interpreted as the Olduvai subchron. The next, poorly defined transition from reversed to normal may be the Matuyama/Gauss boundary at $2.47 \mathrm{Ma}$ (Ness et al., 1980). The initial polarity of Core 3 is interpreted as reversed only because of its length. If this interpretation is accurate, Core 3 is of Gilbert age and the older part of the Gauss Chron (between Cores 2 and 3) is missing. Consequently, the transition at the lower part of Core 3 may be the younger boundary of the Cochiti subchron at $3.86 \mathrm{Ma}$, and Core 4 may contain the four normal subchrons of the Gilbert Chron. The Gilbert/Chron 5 boundary can be identified in Core 5, and the Chron 5/ Chron 6 boundary can be identified in Core 6. Because the older normal polarity interval of Chron 5 is very short, however, material might have been lost between Cores 5 and 6 . From the lower part of Core 6 down to Core 9 (lithologic Subunit IB), the low intensity precludes the determination of any polarity change from either the inclination or declination data, as in Hole 574 (Fig. 8B). Cores 9 and 10 are interpreted as containing the Chron $8 / C$ Chron 9 transition and parts of Chron 9 . The data from the lower part of Core 10 are poor and must be interpreted by extrapolation, as for Hole 574 . Table 3 shows the polarity changes in Holes 574 and 574A by depth and age (Ness et al., 1980). The possible reasons for the differences in depth of some polarity transitions are discussed above.

\section{Site 575}

Site 575 , at $5^{\circ} 51.00^{\prime} \mathrm{N}, 135^{\circ} 02.16^{\prime} \mathrm{W}$, constitutes the northernmost of three sites drilled in a north-south transect across the equatorial high-productivity belt. Miocene to Quaternary sediments were recovered at this site; basement was not reached. The entire set of cores was sampled for paleomagnetic analysis, and a magnetostratigraphy into the lower Miocene was established, although unfortunately it has some gaps due to weak magnetization in the lower to middle Miocene (Fig. 9). The intensities throughout lithologic Unit I at Holes 575, 575B, and $575 \mathrm{C}$ show large-scale variations ranging from $10^{-7}$ $\mathrm{G}$ to about $10^{-6} \mathrm{G}$, with constant averages. The magnetization properties of the Site 575 sediments are similar to those at the other sites. The lower Miocene sequence, which is characterized by a pale brown ooze (lithologic Subunit IIA), provided intensities with a mean of 8.97 $\pm 7.81 \times 10^{-6} \mathrm{G}$.

\section{Hole 575}

If we assume that no polarity boundaries occur between cores, the magnetostratigraphic interpretation of the data for Hole 575 is straightforward. We correlate the uppermost polarity sequence with the Brunhes Normal Chron (Table 4A; Fig. 9A), a correlation supported by the inclination data. Lower we distinguish the Matuyama and Gauss Chrons, as documented by the declination data and, at $10.27 \mathrm{~m}$ sub-bottom, the Gilbert/ Chron 5 transition (5.41 Ma; Ness et al., 1980), in agreement with the biostratigraphic results (Barron et al., this volume). Thus, the entire sequence of the Gilbert normal subchrons appears to be missing as the result of a hiatus. The normal polarity interval ranging from 10.27 to $13.17 \mathrm{~m}$ sub-bottom is interpreted as Chron 5. The magnetic record of the underlying normal polarity sequence (that from 16.15 to $22.77 \mathrm{~m}$ sub-bottom) is poor because of the disturbance of the sediments. The transition at $22.77 \mathrm{~m}$ appears to be the Chron 7/Chron 8 boundary (at $8.18 \mathrm{Ma}$; Ness et al., 1980). We assume that most of Chron 9 is missing as the result of a prominent hiatus (see Barron et al., this volume), but otherwise the polarity record down to the upper part of Chron 12 (Core 4) is reasonably well developed (Fig. 9A, Table $4 \mathrm{~A})$.

\section{Hole 575C}

The two cores that constitute Hole 575C provide an excellent record of the upper Miocene to Quaternary polarity sequence (Fig. 9C, Table 4C). As clearly indicated by the inclination data, the uppermost sequence of uniform declination can be interpreted as the Brunhes Chron. The Brunhes/Matuyama boundary occurs at $2.61 \mathrm{~m}$ subbottom in Core 1 and is followed by the Jaramillo and Olduvai subchrons (Table 4C). Between the termination and the onset of the Gauss Chron, both the Kaena and Mammoth subchrons are present (Fig. 9C; Table 4C). As in Hole 575 (see above), the polarity transition following the Gauss/Gilbert boundary (Core 575C-2; $9.91 \mathrm{~m}$ ) 

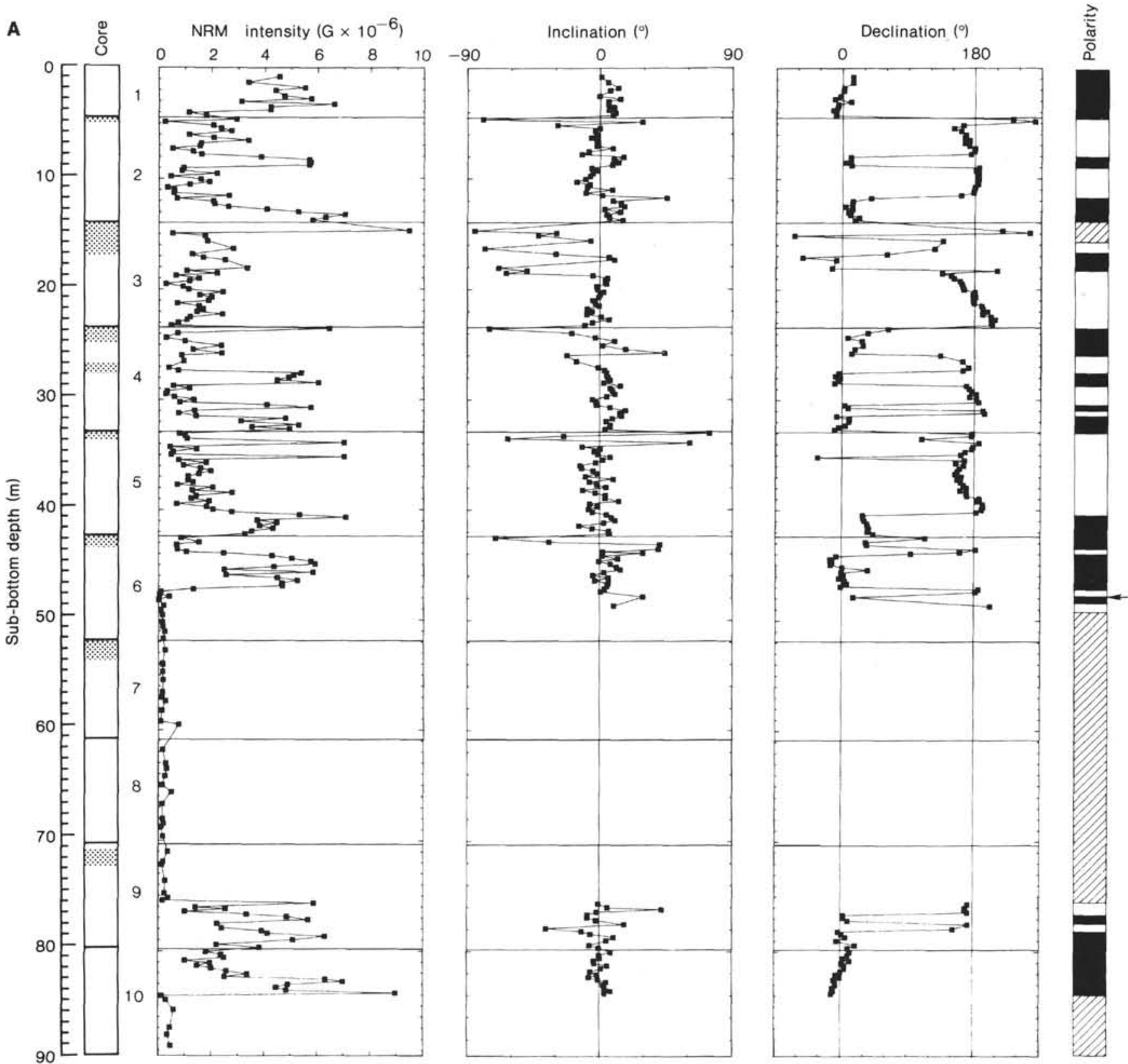

Figure 8. NRM intensity and paleomagnetic polarity logs for (A) Hole 574 and (B) Hole 574A. In the column showing the depths of the cores, dotted intervals are those identified on the barrel sheets as disturbed by drilling. The remaining columns show the downhole variation of NRM intensity; stable inclination and corrected declination (see text for details); and paleomagnetic polarity (derived from $180^{\circ}$ shifts in the declination data). Black indicates intervals of normal polarity and white intervals of reversed polarity. Striping shows intervals where only erratic and $\mathrm{NRM}_{0}$ data were available; these intervals were not interpreted. Arrows indicate polarity changes documented by one data point only.

is correlative to the Gilbert/Chron 5 boundary, a correlation that requires there to be a hiatus during the Gilbert Chron. All the polarity transitions in Chron 5 can be identified (Fig. 9C). The erratic $180^{\circ}$ shifts in the declination data that occur in the lower normal interval of Chron 5 are caused by volcanic detritus (see site chapters, this volume). The polarity change at $15.57 \mathrm{~m}$ sub-bottom (Core 575C-2) agrees well with the N-R transition dated at 6.42 Ma (Ness et al., 1980).

\section{Hole 575B}

In contrast, the record of late Miocene to Quaternary magnetostratigraphy in Hole 575B is poor (Fig. 9B).
Core 575B-1 begins in the Brunhes Chron. The Brunhes/Matuyama boundary at $4.96 \mathrm{~m}$ sub-bottom is followed by the Jaramillo subchron (Fig. 9B). At $6.41 \mathrm{~m}$ the sampling of the first core was discontinued because of the severe disturbance of the sediments. Sampling was resumed at $12.81 \mathrm{~m}$, at the top of Core 2, within an interval of normal polarity as indicated by the inclination data. The uppermost polarity change in Core 2, at $13.06 \mathrm{~m}$, is correlated to the Gauss/Gilbert boundary (3.40 Ma). Since we assumed that there is a hiatus centered on the Gilbert Chron (see discussion above), the two intervals of normal polarity that follow are interpreted as Chron 5. Its upper boundary (5.41 Ma) occurs 


\section{B}

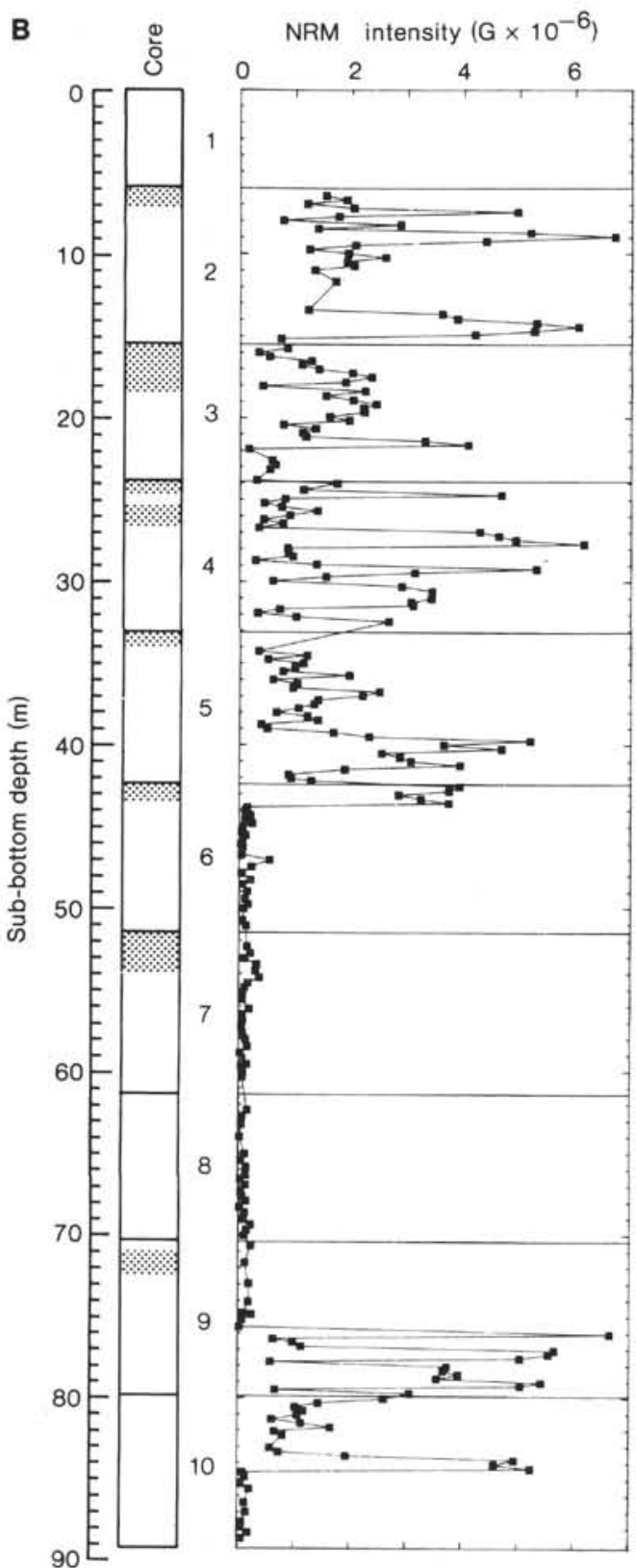

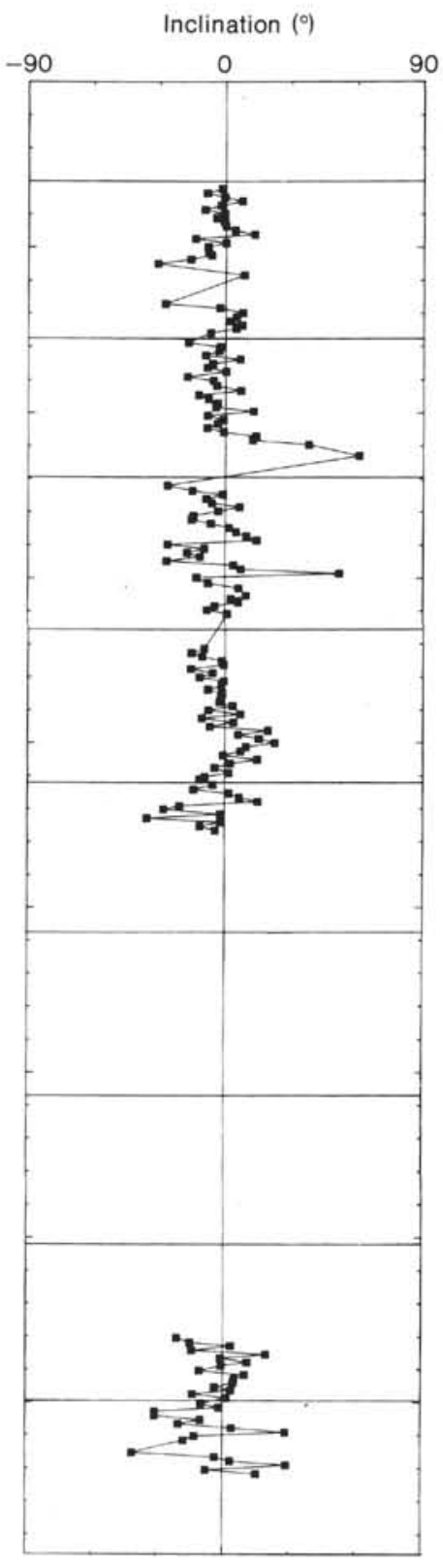

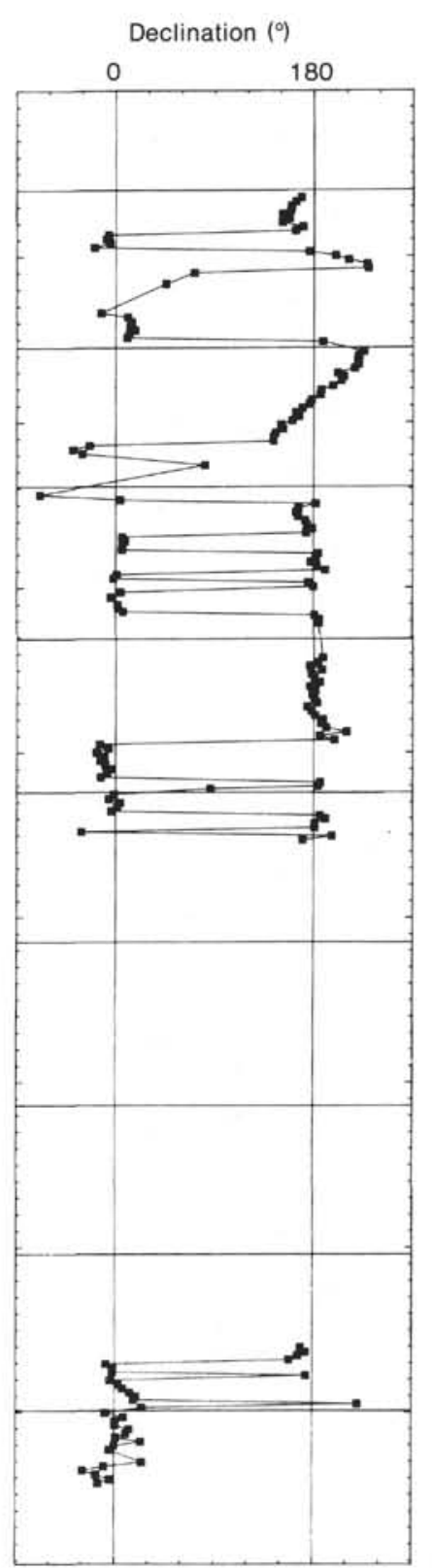

충

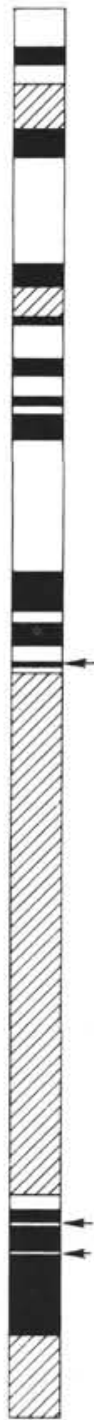

Figure 8. (Continued).

at $14.68 \mathrm{~m}$, and its lower boundary occurs at $18.18 \mathrm{~m}$ sub-bottom. Erratic declinations occur in the lower interval of normal polarity of Chron 5 , in a position stratigraphically correlative with those that occur in Core $575 \mathrm{C}-2$ and for the same reason (volcanic detritus). The polarity change at $21.03 \mathrm{~m}$ sub-bottom in Core 575B-2 correlates with the N-R transition in Hole $575 \mathrm{C}$ that is dated at 6.42 Ma (Ness et al., 1980). The first $180^{\circ}$ shift of declination identified in Core 575B-3 at $22.70 \mathrm{~m}$, therefore, appears to be the transition at $6.55 \mathrm{Ma}$. Unfortunately, further magnetostratigraphic interpretation is impossible because of the weak intensities and erratic inclination and declination data that characterize the underlying sediments.

A comparison of the depths of polarity transitions in Holes 575C and 575B indicates a depth displacement.
Stratigraphically correlative intervals bounded by $180^{\circ}$ shifts in declination (those from the Brunhes/Matuyama boundary down to the Chron 5/Chron 6 boundary and those in the intervals of volcanic detritus) were used to calculate a regression with a correlation coefficient, $r^{2}$, of 0.997 . This displacement between the holes amounts to $1.63 \mathrm{~m}$, instead of the $3.30 \mathrm{~m}$ suggested by the coring logs (which show an offset of that amount between the mudline and the top of Core 575B-1). The determination of the depth of the top of each core was made difficult by the presence of both this within-hole displacement and the overcoring technique used in Holes 575B and 575A (see Site 575 chapter). For this study the length of the interval that was overcored (which is called washdown in the barrel sheets) was subtracted from the length of each core. The good correlation between the polarity 
Table 3. Magnetostratigraphy for Holes 574 and 574A.

\begin{tabular}{|c|c|c|c|c|c|}
\hline \multirow[b]{2}{*}{ Boundary } & \multirow[b]{2}{*}{$\begin{array}{c}\text { Age } \\
(\mathrm{Ma})^{\mathrm{a}}\end{array}$} & \multicolumn{2}{|c|}{ Hole 574} & \multicolumn{2}{|c|}{ Hole $574 \mathrm{~A}$} \\
\hline & & $\begin{array}{c}\text { Depth of } \\
\text { boundary } \\
\text { (m sub-bottom) }\end{array}$ & $\begin{array}{l}\text { Uncertainty range } \\
\text { (Core-Section, } \\
\text { level in } \mathrm{cm})^{\mathrm{c}}\end{array}$ & $\begin{array}{c}\text { Depth of } \\
\text { boundary } \\
(\mathrm{m} \text { sub-bottom) }\end{array}$ & $\begin{array}{l}\text { Uncertainty range } \\
\text { (Core-Section, } \\
\text { level in } \mathrm{cm})^{\mathrm{c}^{\mathrm{c}}}\end{array}$ \\
\hline Brunhes/Matuyama & 0.72 & & & & \\
\hline upper Jaramillo & 0.91 & & & & \\
\hline lower Jaramillo & 0.97 & & & & \\
\hline upper Olduvai & 1.66 & 7.92 & $2-3,30 / 2-3,55$ & 8.58 & $2-2,66 / 2-2,9$ \\
\hline lower Olduvai & 1.87 & 8.92 & $2-3,130 / 2-4,5$ & 9.59 & $2-3,16 / 2-3,42$ \\
\hline Matuyama/Gauss & 2.47 & 11.67 & $2-5,105 / 2-5,130$ & 12.10 & $2-3,142 / 2-5,110$ \\
\hline upper Kaena & 2.91 & & & 15.02 & $2-6,110 / 2-6,135$ \\
\hline lower Kaena & 2.98 & & & & \\
\hline upper Mammoth & 3.07 & & & & \\
\hline lower Mammoth & 3.17 & 16.89 & $3-2,88 / 3-3,40$ & & \\
\hline Gauss/Gilbert & 3.40 & 18.46 & $3-3,113 / 3-4,30$ & & \\
\hline upper Cochiti & 3.86 & 23.86 & $3-7,30 / 4-1,92$ & 21.28 & $3-4,115 / 3-4,141$ \\
\hline lower Cochiti & 3.98 & 26.32 & $4-2,49 / 4-3,65$ & 24.83 & $4-1,81 / 4-1,104$ \\
\hline upper Nunivak & 4.12 & 27.52 & $4-3,90 / 4-3,115$ & 26.82 & $4-2,130 / 4-3,5$ \\
\hline lower Nunivak & 4.26 & 28.67 & $4-4,55 / 4-4,80$ & 27.83 & $4-3,81 / 4-3,105$ \\
\hline upper Sidufjall & 4.41 & 30.42 & $4-5,80 / 4-5,105$ & 29.08 & $4-4,55 / 4-4,81$ \\
\hline lower Sidufjall & 4.49 & 30.92 & $4-5,130 / 4-6,5$ & 29.57 & $4-4,105 / 4-4,130$ \\
\hline upper Thvera & 4.59 & 31.47 & $4-6,30 / 4-6,55$ & 30.12 & $4-5,5 / 4-5,38$ \\
\hline lower Thvera & 4.79 & 33.57 & $4-7,28 / 5-1,135$ & 31.57 & $4-6,6 / 4-6,28$ \\
\hline Gilbert/Chron 5 & 5.41 & 40.42 & $5-5,130 / 5-6,5$ & 39.38 & $5-5,15 / 5-5,41$ \\
\hline Chron 5/Chron 6 & 6.07 & 47.17 & $6-4,5 / 6-4,30$ & 43.69 & $6-1,116 / 6-1,142$ \\
\hline \multicolumn{6}{|c|}{ Core 6 to Core 9 data did not allow identification of specific reversals } \\
\hline Chron 8/Chron 9 & 8.98 & 78.22 & $9-6,10 / 9-6,35$ & 76.94 & $9-5,42 / 9-5,67$ \\
\hline
\end{tabular}

boundaries in Holes 575C and 575B (if the offset of Hole 575B is disregarded) supports this procedure.

\section{Hole 575A}

We could develop a reasonable magnetostratigraphy for only the uppermost 10 cores of Hole 575A. Overcoring was also used in this hole, so the actual core lengths were recalculated as described above. The stratigraphy was derived by defining the mean declination for each core. This mean was then used to arbitrarily align each core's declination with either $0^{\circ}$ (normal polarity) or $180^{\circ}$. Figure 9D shows the resulting polarity pattern. We emphasize, however, that without usable inclination data it is impossible to define the polarity of the sequence on paleomagnetic grounds alone. The interpretation shown in Figure 9D was guided by the general biostratigraphic age of the sediments. Because of the age range of the interval (about 16 to $18 \mathrm{Ma}$ ), this interpretation of polarity also fits the anomaly pattern much better than would the opposite polarity solution. The uppermost sequence with uniform declination data, which starts at $93.80 \mathrm{~m}$ sub-bottom (the top of Core 1 ) was arbitrarily set at $0^{\circ}$ or normal polarity. The three intervals of normal polarity that can be identified in Cores 1 to 3 are interpreted as Anomaly 5C (Mankinen and Dalrymple, 1979; Ness et al., 1980), which begins at $102.64 \mathrm{~m}$ sub-bottom $(16.71 \mathrm{Ma})$. At the bottom of Core 5 (113.54 $\mathrm{m})$ there is a $180^{\circ}$ shift to $0^{\circ}$, and at the top of Core 6 $(114.59 \mathrm{~m})$ the declination shifts back to $180^{\circ}$. This normal polarity interval cannot be correlated to the geomagnetic time scale (Ness et al., 1980). The transition at $118.09 \mathrm{~m}$ sub-bottom is interpreted as the younger limit of Anomaly 5D (17.28 Ma), and the polarity change at $125.59 \mathrm{~m}$ (17.60 Ma; Ness et al., 1980) is interpreted as its older limit. This interpretation agrees well with the accepted time scales and the biostratigraphy (Barron et al., this volume); and it also yields reasonable sedimentation rates for the lower Miocene.

\section{Discussion of Magnetostratigraphy}

The magnetostratigraphy of the Leg 85 sites is summarized in Figure 10. It was derived from the polarity boundaries of each hole by using the time scale of Ness et al. (1980) as a reference. Alternative geomagnetic time scales (McDougall, 1977; Mankinen and Dalrymple, 1979) would produce no significant differences in age for the late Neogene. The sedimentation rates defined by this magnetostratigraphy decrease from the south (Site 573) to the north (Site 575). The northward motion of the Pacific Plate and the migration of the sites into the highproductivity belt of the equatorial region (Winterer, 1973; van Andel et al., 1975) is generally confirmed by the increase in sedimentation rate that occurs as depth in Sites 574 and 575 increases.

The sedimentation rates at Site 573 are surprisingly constant at about $18.2 \mathrm{~m} / \mathrm{m}$.y. At Site 574 , the rates change significantly at approximately $3 \mathrm{Ma}$ (during the Gauss Chron), from about $5.5 \mathrm{~m} / \mathrm{m}$.y. above to about $12.2 \mathrm{~m} / \mathrm{m}$.y. below. Below the Chron 5/Chron 6 boundary, the sedimentation rates are poorly documented, however, because we lack reliable data. There should be a significant hiatus at about 9 to $10 \mathrm{Ma}$ (Moore et al., 1978; Barron and Keller, 1972). The oldest polarity change determined at this site (at about $80 \mathrm{~m}$ sub-bottom) is interpreted here as the Chron $8 / \mathrm{Chron} 9$ boundary. This interpretation disagrees with the biostratigraphic dating, which places the Chron $10 /$ Chron 11 boundary at this level (Barron et al., this volume). Site 575 shows a more 
Table 4A. Magnetostratigraphy for Hole 575.

\begin{tabular}{|c|c|c|c|}
\hline Boundary & $\begin{array}{l}\text { Age } \\
(\mathrm{Ma})^{\mathrm{a}}\end{array}$ & $\begin{array}{c}\text { Depth of } \\
\text { boundary } \\
\text { (m sub-bottom) }\end{array}$ & $\begin{array}{l}\text { Uncertainty range } \\
\text { (Core-Section, } \\
\text { level in } \mathrm{cm})^{\mathrm{c}}\end{array}$ \\
\hline Brunhes/Matuyama & 0.72 & 2.70 & $1-2,115 / 1-2,125$ \\
\hline upper Jaramillo & 0.91 & 3.10 & $1-3,5 / 1-3,15$ \\
\hline lower Jaramillo & 0.97 & 3.20 & $1-3,15 / 1-3,25$ \\
\hline upper Olduvai & 1.66 & 4.10 & $1-3,105 / 1-3,115$ \\
\hline lower Olduvai & 1.87 & 4.30 & $1-3,125 / 1-3,135$ \\
\hline Matuyama/Gauss & 2.47 & 5.40 & $1-4,85 / 1-4,95$ \\
\hline upper Kaena & 2.91 & 6.60 & $1-5,55 / 1-5,65$ \\
\hline lower Kaena & 2.98 & 6.90 & $1-5,85 / 1-5,95$ \\
\hline upper Mammoth & 3.07 & 7.19 & $1-5,115 / 1-5,123$ \\
\hline lower Mammoth & 3.17 & 7.31 & $1-5,128 / 2-1,4$ \\
\hline Gauss/Gilbert & 3.40 & 7.65 & $2-1,30 / 2-1,40$ \\
\hline Gilbert/Chron 5 & 5.41 & 10.27 & $2-2,140 / 2-3,4$ \\
\hline \multirow[t]{2}{*}{ Chron 5/Chron 6} & 6.07 & 13.17 & $2-4,128 / 2-4,145$ \\
\hline & 6.42 & 16.15 & $2-6,130 / 2-6,140$ \\
\hline \multirow[t]{7}{*}{ Chron $7 /$ Chron 8} & 8.18 & 22.77 & $3-4,139 / 3-5,5$ \\
\hline & 8.40 & 25.80 & $3-6,140 / 3-7,10$ \\
\hline & 8.49 & 26.05 & $3-7,10 / 3-7,20$ \\
\hline & 10.42 & 26.85 & $4-1,50 / 4-1,60$ \\
\hline & 10.47 & 27.11 & $4-1,70 / 4-1,91$ \\
\hline & 10.89 & 28.00 & $4-2,7 / 4-2,3$ \\
\hline & 10.96 & 28.42 & $4-2,33 / 4-2,90$ \\
\hline \multirow[t]{3}{*}{ Chron $10 /$ Chron 11} & 11.42 & 29.32 & $4-2,134 / 4-3,20$ \\
\hline & 11.58 & 29.75 & $4-3,40 / 4-3,50$ \\
\hline & 11.71 & 30.65 & $4-3,130 / 4-3,140$ \\
\hline \multirow[t]{5}{*}{ Chron $11 /$ Chron 12} & 11.98 & 31.50 & $4-4,65 / 4-4,75$ \\
\hline & 12.28 & 32.10 & $4-4,125 / 4-4,135$ \\
\hline & 12.32 & 32.67 & $4-5,9 / 4-5,65$ \\
\hline & 12.40 & 33.43 & $4-5,91 / 4-5,134$ \\
\hline & 12.45 & 34.24 & $4-6,39 / 4-6,48$ \\
\hline
\end{tabular}

a Ness et al. (1980).

b Interpolated from coring summary (site chapter).

c Last sample of upper polarity sequence/first sample of lower polarity sequence.

Table 4B. Magnetostratigraphy for Hole 575B.

\begin{tabular}{lccc}
\hline \multicolumn{1}{c}{ Boundary } & $\begin{array}{c}\text { Age } \\
(\mathrm{Ma})^{\mathrm{a}}\end{array}$ & $\begin{array}{c}\text { Depth of } \\
\text { boundary } \\
\text { (m sub-bottom) }\end{array}$ & $\begin{array}{c}\text { Uncertainty range } \\
\text { (Core-Section, }^{\text {level in cm) }}\end{array}$ \\
\hline Brunhes/Matuyama & 0.72 & 4.96 & $1-2,11 / 1-2,21$ \\
upper Jaramillo & 0.91 & 5.46 & $1-2,61 / 1-2,71$ \\
lower Jaramillo & 0.97 & 5.72 & $1-2,71 / 1-2,113$ \\
Gauss/Gilbert & 3.40 & 13.06 & $2-1,101 / 1-1,111$ \\
Gilbert/Chron 5 & 5.41 & 14.68 & $2-2,113 / 2-2,123$ \\
& 5.70 & 15.75 & $2-3,73 / 2-3,83$ \\
Chron 5/Chron 6 & 5.78 & 16.58 & $2-4,3 / 2-4,13$ \\
& 6.07 & 18.18 & $2-5,13 / 2-5,23$ \\
& 6.42 & 21.03 & $2-6,143 / 2-7,13$ \\
& 6.55 & 22.70 & $3-2,9 / 3-2,31$ \\
\hline
\end{tabular}

a Ness et al. (1980).

b Interpolated from coring summary (site chapter) and corrected with regard to each core's washdown.

c Last sample of upper polarity sequence/first sample of lower polarity sequence.

d Correlation with the known polarity sequence was not possible in the rest of Core 3 or in Core 4 .

complex pattern of accumulation rates. The rates are very low at the top (less than $3 \mathrm{~m} / \mathrm{m}$.y.), and there are two possible hiatuses. The youngest prominent hiatus seems to occur between about 3.6 and 5.3 Ma (Barron et al., this volume), so almost the entire Gilbert Chron may be missing at this site. The magnetic data from about 16 to $23 \mathrm{~m}$ sub-bottom $(\approx 6.4 \mathrm{Ma})$ are uncertain because of coring disturbance. Shipboard biostratigraphy suggests that a second hiatus affects the record of Chron 9. If so, the polarity pattern of Chrons 10 to 12
Table 4C. Magnetostratigraphy for Hole 575C.

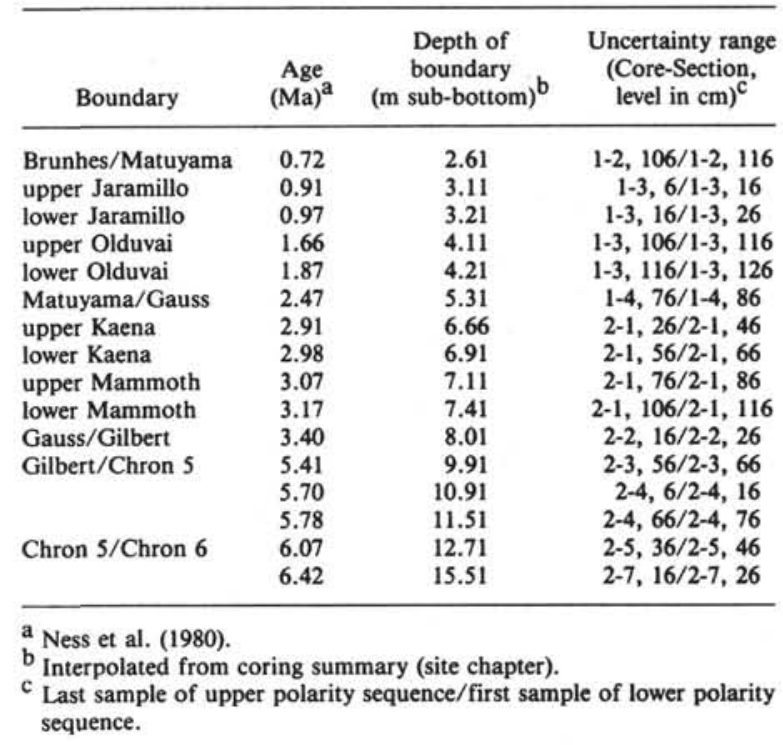

Table 4D. Magnetostratigraphy for Hole 575A.

\begin{tabular}{lccc}
\hline Boundary & $\begin{array}{c}\text { Age } \\
(\mathrm{Ma})^{\mathrm{a}}\end{array}$ & $\begin{array}{c}\text { Depth of } \\
\text { boundary } \\
\text { (m sub-bottom) }^{\mathrm{b}}\end{array}$ & $\begin{array}{c}\text { Uncertainty range } \\
\text { (Core-Section, }_{\text {level in cm) }}{ }^{\mathrm{c}}\end{array}$ \\
\hline & 16.25 & 95.79 & $1-1,29 / 1-1,69$ \\
& 16.29 & 97.29 & $1-3,29 / 1-3,69$ \\
Anomaly SC onset & 16.46 & 99.69 & $2-1,49 / 2-1,109$ \\
& 16.53 & 101.74 & $2-2,109 / 2-3,9$ \\
Anomaly 5D termination & 16.71 & 102.64 & $3-1,9 / 3-1,59$ \\
Anomaly 5D onset & $?$ & 113.59 & $5-3,39 / 5-3,97$ \\
& 17.28 & 114.59 & $6-1,29 / 6-1,69$ \\
& 17.60 & 118.09 & $6-3,69 / 6-3,129$ \\
& 17.81 & 125.59 & $8-2,49 / 8-2,109$ \\
& 17.83 & 128.44 & $10-1,1 / 10-1,46$ \\
& & 129.83 & $10-1,134 / 10-2,41$ \\
\hline
\end{tabular}

a Ness et al. (1980).

$\mathrm{b}$ Interpolated from coring summary (site chapter) and corrected with regard to each core's washdown.

${ }^{c}$ Last sample of upper polarity sequence/first sample of lower polarity sequence.

correlates with the measured magnetostratigraphy. From about $34.5 \mathrm{~m}$ to $93.8 \mathrm{~m}$ sub-bottom, very weak intensities again precluded the development of a coherent magnetostratigraphy. The polarity pattern established throughout the upper 10 cores of Hole 575A (Fig. 9D) is interpreted here as being correlated with Anomalies 5C and 5D (about 16 to $18 \mathrm{Ma}$; Ness et al., 1980), an interpretation that is generally compatible with biostratigraphic results (Barron et al., this volume). Despite the poor control, an increase in sedimentation rates up to $21.5 \mathrm{~m} / \mathrm{m}$.y. can be detected in the older sediments.

The transition at all Leg 85 sites from cyclic siliceouscalcareous oozes to highly calcareous oozes is accompanied by a remarkable decrease in NRM intensities that may be caused by a significant reduction in the proportion of magnetic grains in the Miocene and older sediments. It is more likely, however, that the magnetic carriers have been altered (Kent and Lowrie, 1974; Henshaw and Merrill, 1980). The alteration may be related to the change from mainly calcareous (foram-nanno) 
A

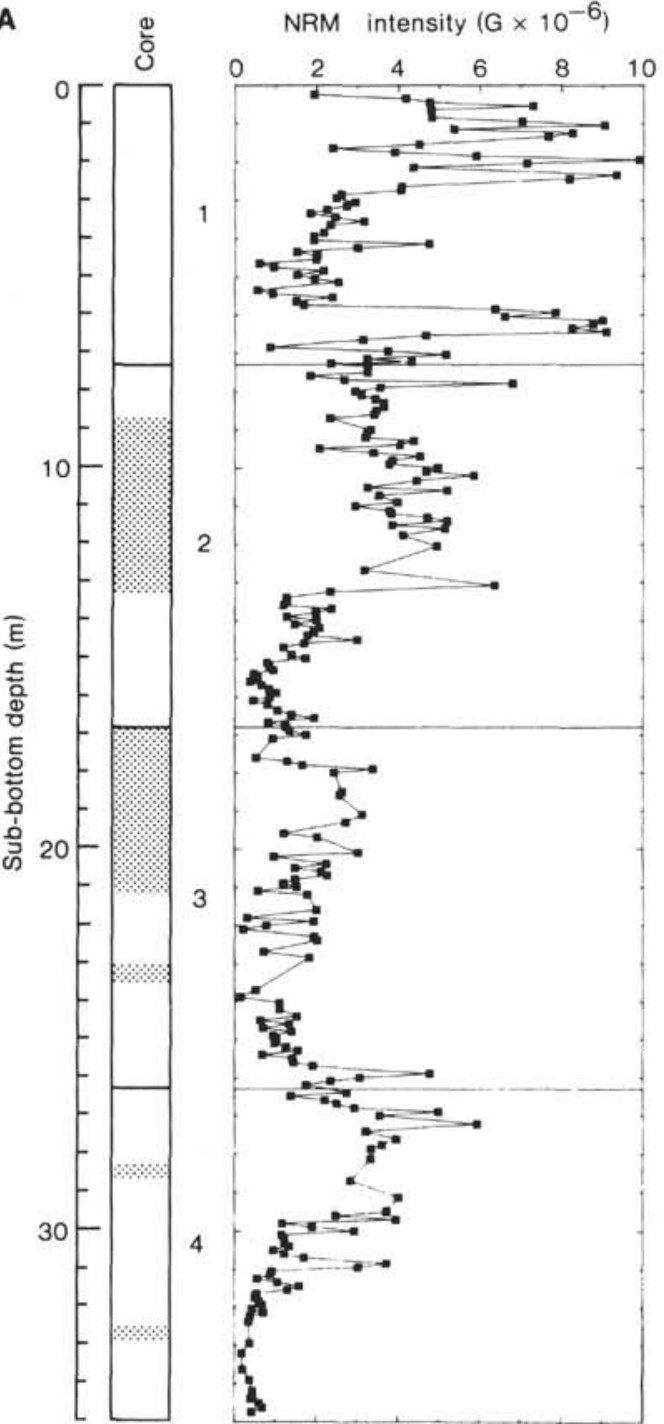

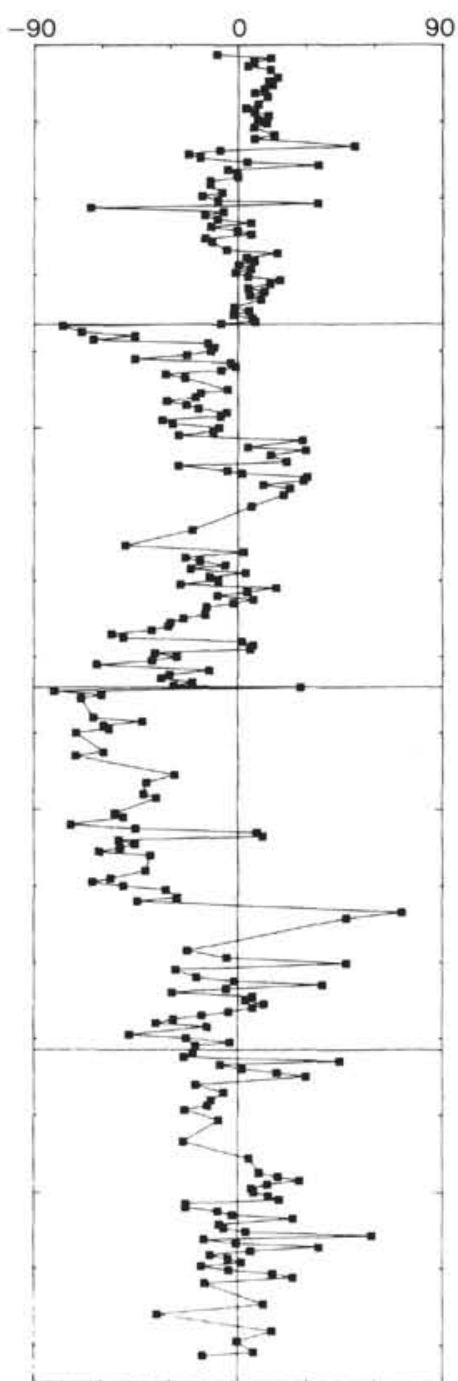
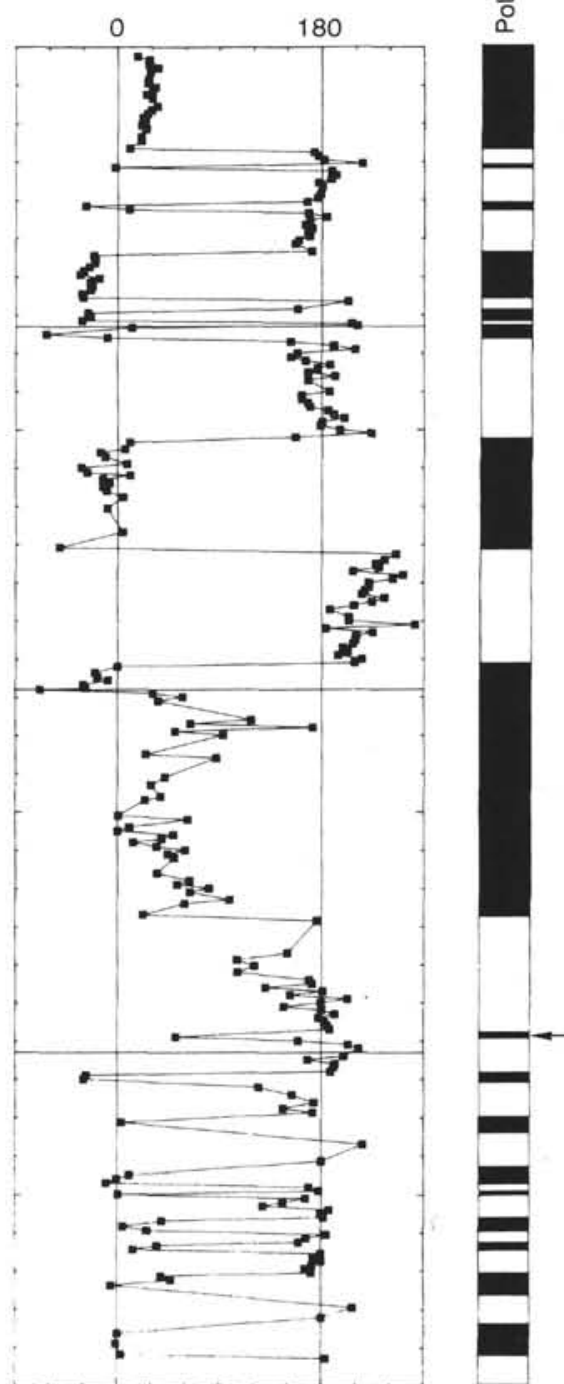

Figure 9. Polarity and NRM intensity logs for (A) Hole 575, (B) 575B, (C) Hole 575C, and (D) Hole 575A. See Figure 8 caption for further explanation.

oozes below to more siliceous sediments above or to diagenetic changes that begin at some 40 to $80 \mathrm{~m}$ sub-bottom at these sites. It is possible, of course, that all three of the conditions mentioned above are at work simultaneously. We performed no rock-magnetic analyses, so none of these ideas is supported by experimental evidence. However, Kent (1982) has shown that there is a correlation between low biogenic carbonate and high silica content and high NRM intensity. We noted an empirical relation between large-scale carbonate content variations and NRM intensity changes in the cyclic units of the Leg 85 sites, although no direct correlation was attempted. This relation may reflect a climatically driven, complex interaction between these parameters (Kent, 1982). No prominent changes in sedimentation rate or porosity were noted in these cyclic intervals (site chapters), so the amounts or properties of the magnetic materials (Hamano, 1980) are unlikely to be strongly affected by these factors. In contrast, the poor quality of the data in the metalliferous sediment layers overlying basement at Sites 573 and 574 is in all probability caused by erratic chemical and thermal magnetic overprints due to hydrothermal alteration.

\section{Tectonic Analysis}

The Pacific Plate has moved northwest at the rate of a little less than $1^{\circ} / \mathrm{m}$.y. during the Mesozoic and Cenozoic. Different authors have used various methods to infer this motion. The methods include the study of the thickness of equatorial pelagic facies (Hays et al., 1969; Winterer, 1973; van Andel et al., 1975), basement age comparisons (Hays et al., 1972), hotspot traces (Minster et al., 1974; Epp, 1978; Gordon and Cape, 1981), the study of seamount magnetization (Sager, 1983), the study of magnetic anomaly patterns (Atwater and Menard, 1970; Herron, 1972), and the analysis of the paleomagnetism of central Pacific sedimentary cores (Hammond et al., 1974, 1975, 1979). Although our data are limited, we have attempted to study the northward component of plate motion by using Leg 85 paleomagnetic results. We divided the usable data from Sites 573, 574, and 575 into the time intervals or ranges listed in Table 5. These 

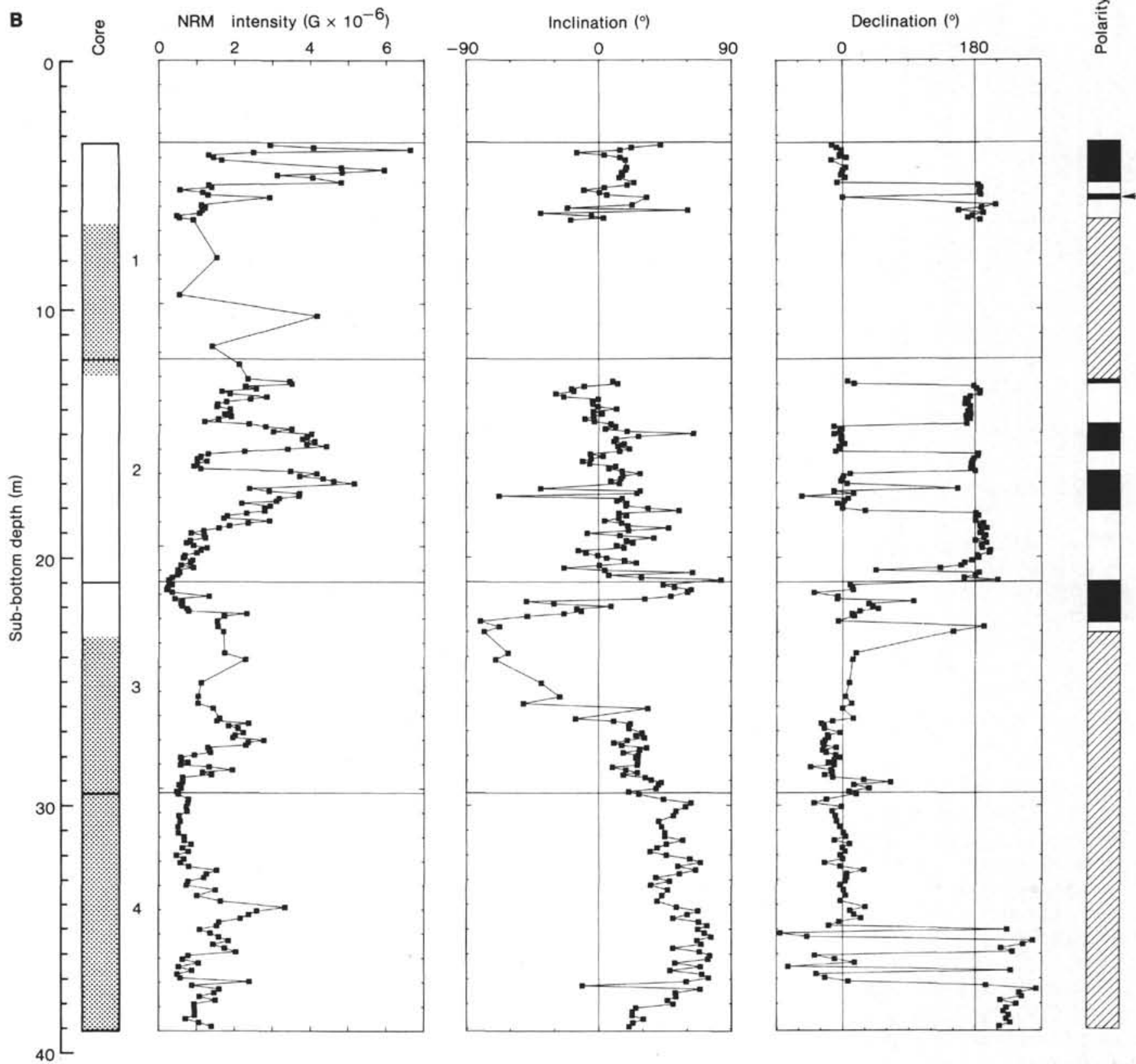

Figure 9. (Continued).

particular intervals were chosen because the resolution of our data varies (resolution is highest in the youngest intervals and lowest in the oldest sediments). Hiatuses, or inferred gaps, also influenced the choices. Thus, the Plio-Pleistocene data for Site 574, for example, were divided at the Gauss/Gilbert boundary into two intervals; an upper Miocene interval was also used. In the data for Site 575 a Pliocene hiatus was used as a boundary between two data sets; and a third interval between about 16 and $18 \mathrm{Ma}$ was also analyzed (see previous discussion of Hole 575A). Using inclination data only, we averaged data from correlative intervals of parallel holes at each site (Fig. 10). We then calculated mean dipole paleolatitudes for each time interval by using statistics developed by Kono (1980), which provide $95 \%$ confidence limits. The mean paleolatitude for each time interval was then normalized for present site latitudes, and a latitude differential, or $\delta$ latitude, was derived. The latter was plotted (Table 5, Fig. 11) against the mean age of the interval as shown in Bleil (1981). The regression coefficient of 0.99 indicates a good linear fit (Fig. 11), and the slope of the curve expresses a northward motion of $0.29^{\circ} / \mathrm{m}$.y., which agrees in general with previous approaches. (Figure 11 also supports our interpretation of the upper Miocene polarity sequence at Site 574. If that interval were as old as suggested by the biostratigraphy [Chron 8], the curve's fit would deteriorate.)

Paleolatitudes for Leg 85 sites were also calculated by using the rotation poles derived from a hotspot analysis (Epp, 1978). The backtracked positions of Sites 573 to 575 are shown in 1-m.y. steps in Figure 12. The hotspot paleolatitudes corresponding to the mean ages of the 

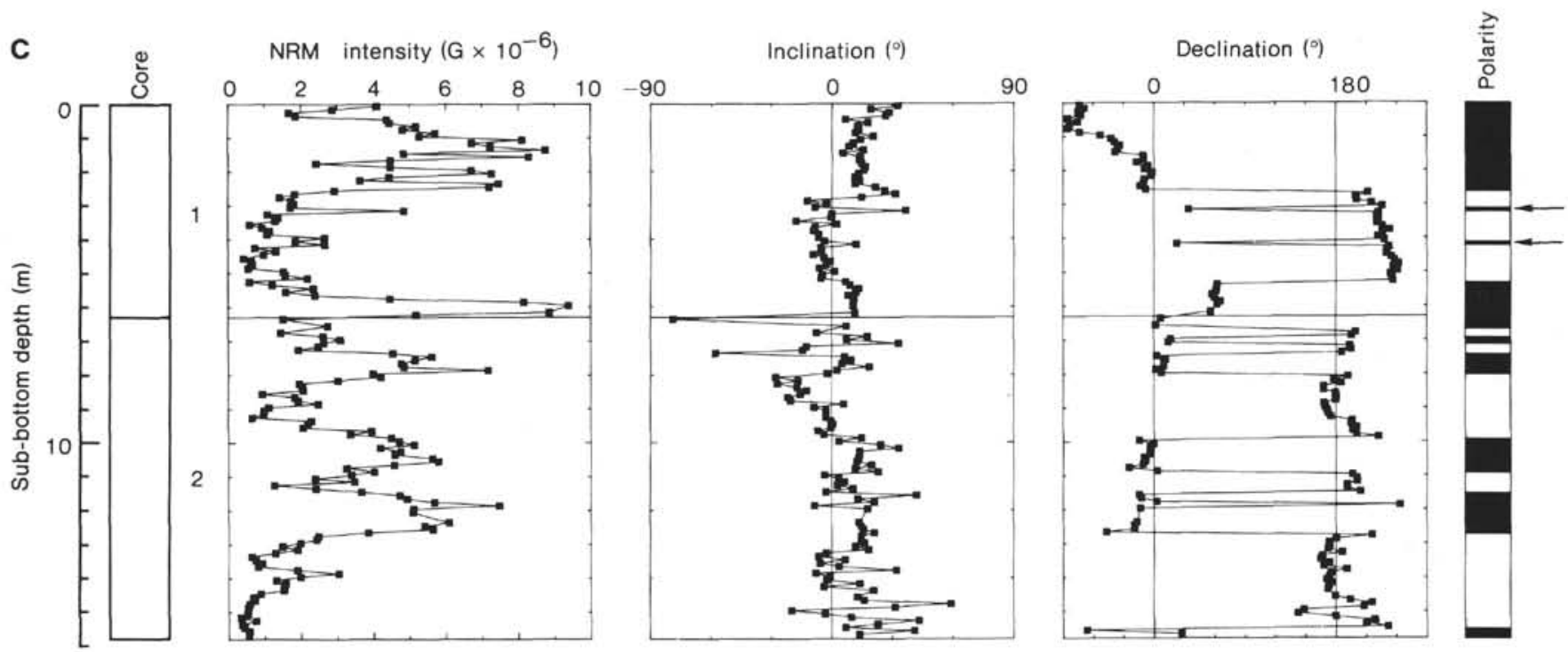

Figure 9. (Continued).

time intervals chosen here are listed in Table 5 together with present site latitude minus dipole paleolatitude and present site latitude minus hotspot paleolatitude. Table 5 shows that the hotspot-based differences are always smaller than those based on paleomagnetic data. If we assume that the hotspot data carry only tectonic information, this difference can be interpreted as an expression of oscillatory far-sided effects of the Earth's magnetic field (Epp et al., 1983). The regression $\left(r^{2}=0.99\right)$ calculated from hotspot data shows a shallower slope $\left(0.23^{\circ} / \mathrm{m}\right.$.y., indicating a slower northward motion for the Pacific Plate) than the slope resulting from a hotspot reference system, a conclusion that Epp et al. (1983) arrived at on the basis of central Pacific piston cores. As the authors pointed out, the differences could also be explained by assuming that the hotspot is not fixed with respect to the Earth's spin axis.

In addition to the above paleolatitude analysis, we also calculated paleo-pole positions for some time intervals. Gordon and Cox developed a technique (1980) that calculates a mean pole from a given model pole by using an iterative least-squares method. With this procedure it is possible to use heterogeneous (unoriented as well as azimuthally oriented) magnetic and paleomagnetic data to define paleo-pole positions. Because the Leg 85 data are confined to virtually one point on the Pacific and thus result in large uncertainties, paleomagnetic data from several HIG conventional piston cores (CPCs) were added. The CPCs, which were acquired 30 to $40^{\circ}$ west of Leg 85 's sites (at similar latitudes), span the same time interval as the Leg 85 data. Most of the CPC data have the advantage of being azimuthally oriented (Seyb et al., 1977), which improves the resolution of the Gordon and Cox (1980) method. We arbitrarily divided the entire data set into three age groups: Recent to about $6 \mathrm{Ma}, 8$ to approximately $11 \mathrm{Ma}$, and 15 to about $18 \mathrm{Ma}$ (Table 6). Paleo-pole positions were then calculated (with Gordon and Cox's procedure) together with their semi-axes of the $95 \%$ oval of confidence (Table 6). These geograph- ically well separated and internally coherent data sets produced relatively small and discrete $95 \%$ confidence ovals, although for the early Miocene pole the data are less numerous and the constraints are correspondingly broader. An apparent polar wander path (APWP) could thus be derived for the interval from 0 to $18 \mathrm{Ma}$ (Fig. 13) that substantiates the overall northwest motion of the Pacific Plate. Our APWP shows a larger longitudinal and latitudinal displacement than the data published by Gordon and Cape (1981) for the Pacific Plate, although the trends are similar. Our paleo-pole latitudes indicate a northward component of motion for the Pacific Plate of about $0.59 \pm 0.08^{\circ} / \mathrm{m}$.y. during the last $18 \mathrm{~m}$.y. This migration exceeds our earlier calculations, which are based on the Leg 85 inclination data alone. One can interpret this difference as reflecting the longitudinal separation (on the same plate) between the Leg 85 sites and the HIG CPCs. Hammond et al. (1979) gave an average northward motion calculated on the basis of inclination data from CPCs (which included the cores used in this paper) of about $0.59^{\circ} / \mathrm{m} . y$. Our tectonic analysis also shows that low-latitude inclination data, previously often considered to be useless, can be employed with significant success for tectonic work. Finally, the study confirms the results of previous work that had pointed out that Tertiary paleomagnetic data may contain appreciable components of long- and short-term paleofield variations in addition to tectonic information (Epp et al., 1983; Theyer et al., 1983).

\section{ACKNOWLEDGMENTS}

We thank all persons responsible for the success of DSDP Leg 85, especially the JOIDES Ocean Paleoenvironmental Panel, who were the original "movers" behind it all. Special appreciation is given to U. Bleil and V. Spiess for discussions and valuable comments. D. Wilson, S. Hammond, D. Epp, and W. Sager, all formerly at HIG, stimulated and helped with the paleomagnetic-tectonic phase. J. Barron and C. Nigrini assisted with stratigraphic data and discussions. N. Shackleton suggested changes in an early version of the manuscript, and two anonymous reviewers offered many suggestions for improvements. $R$. 
D
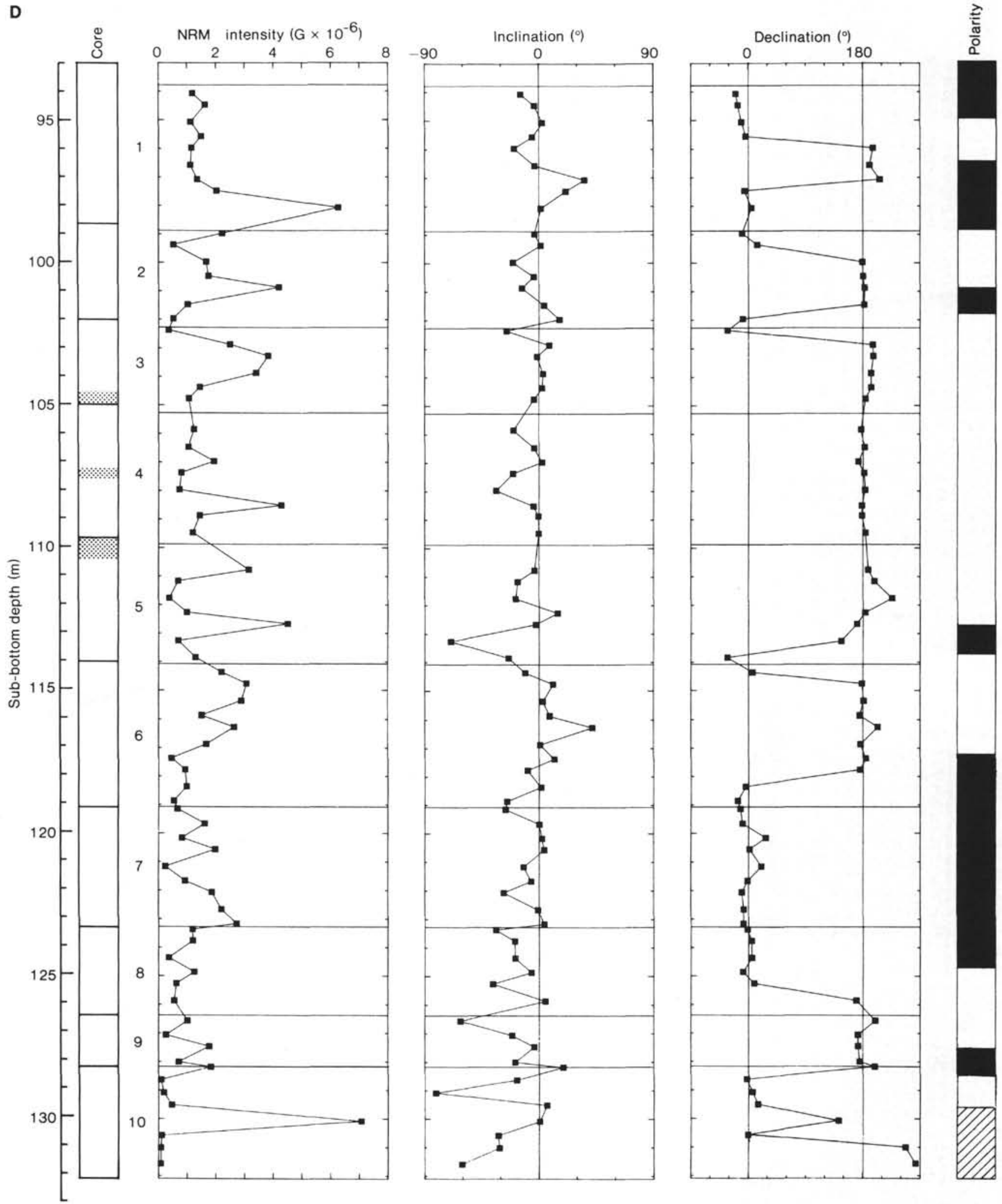

Figure 9. (Continued).

Pujalet also assisted with editorial advice. J. Tuthill helped develop tectonic software. We are also grateful to the members of the HIG Core Analysis Laboratory for their support.

N.W. thanks the Deutsche Forschungsgemeinschaft for support. F.T.'s research was in part supported under National Science Foundation Grant OCE81-17997. The HIG Core Analysis Laboratory is sup- ported under NSF Grant OCE82-0196. This paper is HIG Contribution No. 1463.

\section{REFERENCES}

Atwater, T., and Menard, H. W., 1970. Magnetic lineations in the northwest Pacific. Earth Planet. Sci. Lett., 7:445-450. 
Barron, J. A., and Keller, G., 1982. Widespread Miocene deep-sea hiatuses: coincidence with periods of global cooling. Geology, 10: $577-581$.

Bleil, U., 1981. Paleomagnetism of Deep Sea Drilling Project Leg 60 sediments and igneous rocks from the Mariana region. In Hussong, D. M., Uyeda, S., et al., Init. Repts. DSDP, 60: Washington (U.S. Govt. Printing Office), 855-873.

Epp, D., 1978. Age and tectonic relationships among volcanic chains on the Pacific plate [Ph.D. Dissert.]. University of Hawaii, Honolulu.

Epp, D., Sager, W., Theyer, F., and Hammond, S., 1983. Hotspot/ spin axis motion or far-sided effects? Nature, 303:318-320.

Fisher, R. A., 1953. Dispersion on a sphere. Proc. R. Soc. London, 217:295-305.

Gordon, G. G., and Cape, C. D., 1981. Cenozoic latitudinal shift of the Hawaiian hotspot and its implications for true polar wander. Earth Planet. Sci. Lett., 55:37-47.

Gordon, G. G., and Cox, A., 1980. Calculating paleomagnetic poles for oceanic plates. Geophys. J. R. Astron. Soc., 63:619-640.

Goree, W. S., and Fuller, M., 1976. Magnetometers using RF-driven SQUIDS and their applications in rock magnetism and paleomagnetism. Rev. Geophys. Space Phys., 14:591-608.

Hamano, Y., 1980. An experiment on the post-depositional remanent magnetization in artificial and natural sediments. Earth Planet. Sci. Lett., 51:221-232.

Hammond, S. R., Epp, D., and Theyer, F., 1979. Neogene relative motion between the Pacific plate, the mantle, and the Earth spin axis. Nature, 278:309-312.

Hammond, S. R., Kroenke, L. W., and Theyer, F., 1975. Northward motion of the Ontong-Java plateau between -110 and -30 my: a paleomagnetic investigation of Site 289. In Andrews, J. E., Packham, G., et al., Init. Repts. DSDP, 30: Washington (U.S. Govt. Printing Office), 415-418.

Hammond, S. R., Theyer, F., and Sutton, G. H., 1974. Paleomagnetic evidence of northward movement of the Pacific Plate in deep-sea cores from the central Pacific basin. Earth Planet. Sci. Lett., 22: 22-28.

Hays, J. D., et al., 1972. Init. Repts. DSDP, 9: Washington (U.S. Govt. Printing Office).

Hays, J. D., Saito, T., Opdyke, N. D., and Burckle, L. H., 1969. Pliocene-Pleistocene sediments of the equatorial Pacific: their paleomagnetic, biostratigraphic, and climatic record. Geol. Soc. Am. Bull., 80:1481-1514.

Henshaw, P. C., and Merrill, R. T., 1980. Magnetic and chemical changes in marine sediments. Rev. Geophys. Space Phys., 18: 483-504.

Herron, E. M., 1972. Sea-floor spreading and the Cenozoic history of the east central Pacific. Geol. Soc. Am. Bull., 83:1671-1692.

Hoffman, K. A., and Day, R., 1978. Separation of multi-component NRM, a general method. Earth Planet. Sci. Lett., 40:433-438.

Kent, D. V., 1982. Apparent correlation of paleomagnetic intensity and climatic records in deep-sea sediments. Nature, 299:538-539.

Kent, D. V., and Lowrie, W., 1974. Origin of magnetic instability in sediment cores from the central North Pacific. J. Geophys. Res., 79:2987-3000.

Kent, D. V., and Spariosu, D. J., 1982a. Magnetostratigraphy of equatorial Pacific Site 503 hydraulic piston cores. In Prell, W. L., Gardner, J. V., et al., Init. Repts. DSDP, 68: Washington (U.S. Govt. Printing Office), 435-440.

1982b. Magnetostratigraphy of Caribbean Site 502 hydraulic piston cores. In Prell, W. L., Gardner, J. V., et al., Init. Repts. DSDP, 68: Washington (U.S. Govt. Printing Office), 419-433.
Kono, M., 1980. Statistics of paleomagnetic inclination data. J. Geophys. Res., 85:3878-3882.

Løvlie, R., 1976. The intensity pattern of post-depositional remnance acquired in some marine sediments deposited during a reversal of the external magnetic field. Earth Planet. Sci. Lett., 30:209-214.

McDougall, I., 1977. The Present Status of the Geomagnetic Polarity Time Scale: Austr. Nat. Univ., Res. School Earth Sci. (Canberra), Publ. No, 1288.

Mankinen, E. A., and Dalrymple, G. B., 1979. Revised geomagnetic polarity time scale for the interval 0-5 my B.P. J. Geophys. Res., 84:615-626.

Minster, J. B., Jordan, T. H., Molnar, P., and Haines, E., 1974. Numerical modeling of instantaneous plate tectonics. Geophys. J. R. Astron. Soc., 36:541-576.

Moore, T. C., van Andel, T. H., Sancetta, C., and Pisias, N., 1978. Cenozoic hiatuses in pelagic sediments. Micropaleontology, 24: 113-138.

Ness, G., Levi, S., and Couch, R., 1980. Marine magnetic anomaly timescales for the Cenozoic and Late Cretaceous: a precis, critique, and synthesis. Rev. Geophys. Space Phys., 18:753-770.

Opdyke, N. D., 1972. Paleomagnetism of deep-sea cores. Rev. Geophys. Space Phys., 10:213-249.

Opdyke, N. D., Burckle, L. H., and Todd, A., 1974. The extension of the magnetic time scale in sediments of the central Pacific ocean. Earth Planet. Sci. Lett., 22:300-306.

Petersen, N., and Roggenthen, W. M., 1980. Rock- and paleomagnetism of Deep Sea Drilling Project Leg 54 basalts-East Pacific Rise and Galapagos Rift. In Rosendahl, B. R., Hekinian, R., et al., Init. Repts. DSDP, 54: Washington (U.S. Govt. Printing Office), $865-877$

Sager, W. W., 1983. A late Eocene paleomagnetic pole for the Pacific plate. Earth Planet. Sci. Lett., 63:408-422.

Seyb, S. M., Hammond, S. R., and Gilliard, T., 1977. A new device for recording the behavior of a piston corer. Deep Sea Res., 24 : 943-950.

Tauxe, L., Tucker, P., Petersen, N., and LaBrecque, J., 1983. The magnetostratigraphy of Leg 73 sediments. Palaeogeogr. Palaeclimatol., Palaeoecol., 42:65-90.

Theyer, F., and Hammond, S. R., 1974. Cenozoic magnetic time scale in deep-sea sediments: completion of the Neogene. Geology, 2: 487-492.

Theyer, F., Mato, C. Y., and Hammond, S. R., 1978. Paleomagnetic and geochronologic calibration of latest Oligocene to Pliocene radiolarian events, equatorial Pacific. Mar. Micropaleontol., 3: 377-395.

Theyer, F., Weinreich, N., and Bleil, U., 1983. Neogene polar wander path for the Pacific: plate motion and field behavior. Internat. Union Geol. Geophys. Gral. Assembly, Hamburg. (Abstract)

van Andel, T. H., Heath, G. R., and Moore, T. C., 1975. Cenozoic history and paleoceanography of the central equatorial Pacific Ocean. Mem. Geol. Soc. Am., 143:1-134.

Winterer, E. L., 1973. Sedimentary facies and plate tectonics of equatorial Pacific. Am. Assoc. Pet. Geol. Bull., 57:265-282.

Zijderveld, J. D. A., 1967. AC demagnetization of rocks: analysis of results. In Collison, D. W., Creer, K. M., and Runcorn, S. K. (Eds.), Methods in Paleomagnetism: Elsevier (N.Y.), pp. 254-286.

Date of Initial Receipt: 18 August 1983 Date of Acceptance: 4 April 1984 
Hole

$\begin{array}{llllllll}573 & 573 \mathrm{~A} & 574 & 574 \mathrm{~A} & 575 & 575 B & 575 \mathrm{C}\end{array}$

$\frac{1}{\mathrm{~B},} \mathrm{M}^{\underline{2}}, \frac{2 \mathrm{~A}}{\mathrm{Ga}} \frac{3}{\mathrm{Gi}} \quad \frac{3 \mathrm{~A}}{5,} 6 \frac{4}{1}{ }_{7} \frac{4 \mathrm{~A}}{1}, \frac{5}{9}, 10 \frac{5 \mathrm{~A}}{1111} 121114, \quad 15 \frac{5 \mathrm{~B}}{1} \quad \frac{5 \mathrm{C}}{16}, \frac{5 \mathrm{D}}{17}$ Anomaly

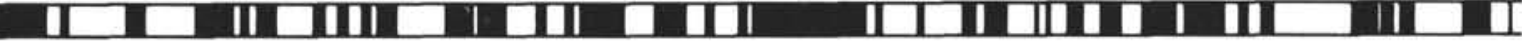
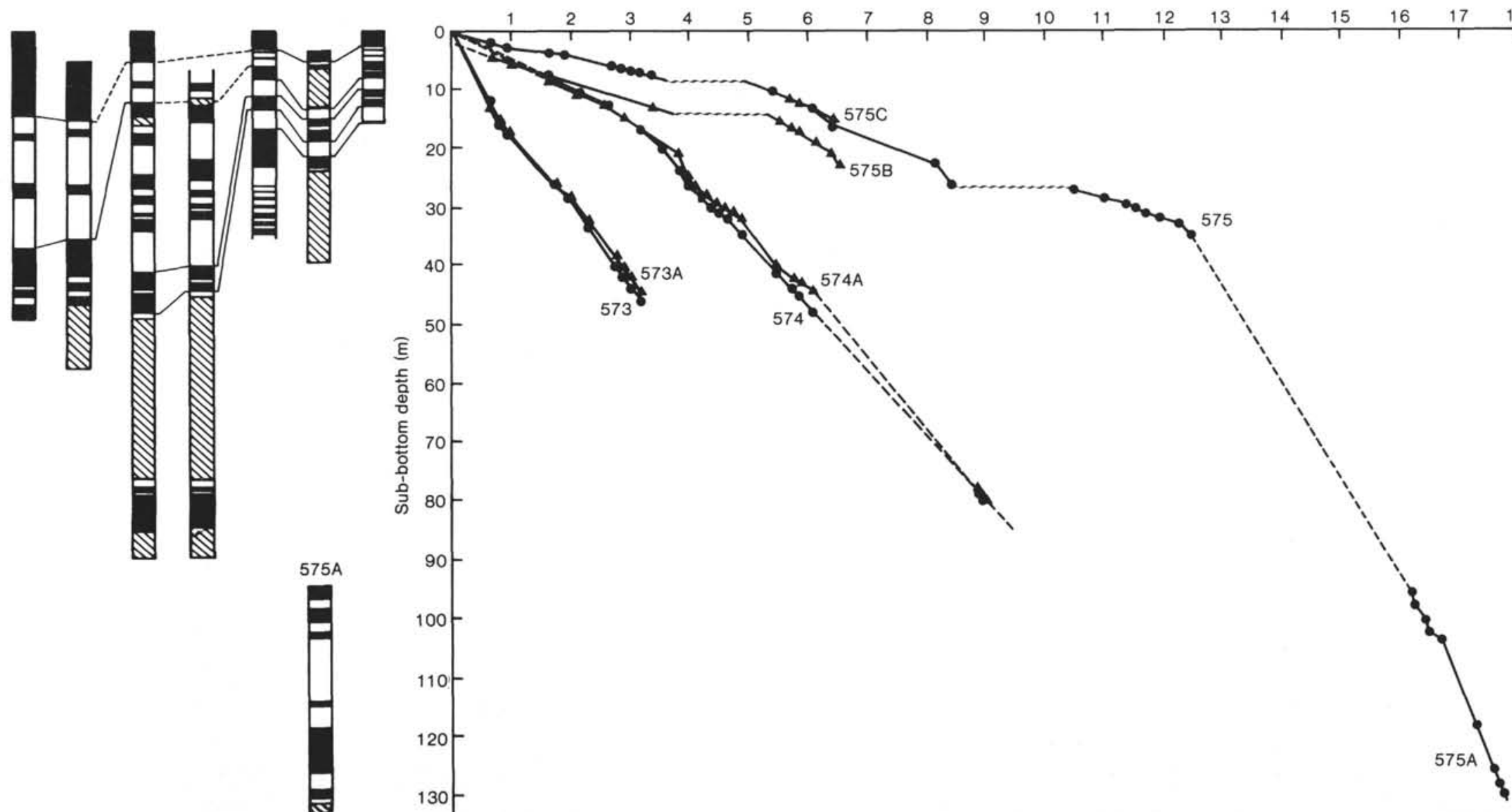

Figure 10. Summary of magnetostratigraphy and sedimentation rates for the sections at Sites 573, 574, and 575. Sediment depth and polarity are plotted against the geomagnetic time scale of Ness et al. (1980). The major marine magnetic anomalies are also shown (Mankinen and Dalrymple, 1979). The circles and triangles in the sedimentation curves distinguish data from different holes. Wavy lines indicate hiatuses. The dashed lines (Sites 574 ar 575) indicate lack of data. Black indicates intervals of normal polarity, white intervals of reversed polarity, and striping intervals that could not be interpreted. $\mathrm{B}=$ Brunhes, $\mathrm{M}=$ Matuyama, $\mathrm{Ga}=$ Gauss, $\mathrm{Gi}=$ Gilbert Chron. 
Table 5. Summary of tectonic parameters.

\begin{tabular}{|c|c|c|c|c|c|c|c|c|c|}
\hline \multirow[b]{2}{*}{ Site } & \multirow[b]{2}{*}{ Location } & \multirow{2}{*}{$\begin{array}{l}\text { Age } \\
\text { range } \\
(\mathrm{Ma})^{\mathrm{a}}\end{array}$} & \multirow{2}{*}{$\begin{array}{l}\text { Arithmetic } \\
\text { mean of } \\
\text { age range } \\
\text { (Ma) }\end{array}$} & \multirow{2}{*}{$\begin{array}{l}\text { Number of } \\
\text { data used to } \\
\text { calculate } \\
\text { paleolatitude }\end{array}$} & \multirow[b]{2}{*}{$\begin{array}{c}\text { Dipole } \\
\text { paleolatitude }^{\mathrm{b}}\end{array}$} & \multirow{2}{*}{$\begin{array}{l}95 \% \\
\text { confidence } \\
\text { limit } \\
\left({ }^{\circ}\right)^{\mathrm{c}}\end{array}$} & \multirow[b]{2}{*}{$\begin{array}{c}\text { Hotspot } \\
\text { paleolatitude }\end{array}$} & \multicolumn{2}{|c|}{$\begin{array}{c}\text { Difference }\left({ }^{\circ}\right) \text { between } \\
\text { present site and }\end{array}$} \\
\hline & & & & & & & & $\begin{array}{c}\text { Dipole } \\
\text { paleolatitude }\end{array}$ & $\begin{array}{c}\text { Hotspot } \\
\text { paleolatitude }\end{array}$ \\
\hline 573 & $0.5^{\circ} \mathrm{N}, 133.3^{\circ} \mathrm{W}$ & $0-3.3$ & 1.7 & 162 & $0.81^{\circ} \mathrm{S}$ & \pm 0.78 & $0.36^{\circ} \mathrm{S}$ & 1.31 & 0.86 \\
\hline \multirow[t]{3}{*}{574} & $4.2^{\circ} \mathrm{N}, 133.3^{\circ} \mathrm{W}$ & $0-3.4$ & 1.7 & 74 & $2.95^{\circ} \mathrm{N}$ & \pm 0.86 & $3.43^{\circ} \mathrm{N}$ & 1.25 & 0.77 \\
\hline & & $3.4-6.2$ & 4.8 & 182 & $1.91^{\circ} \mathrm{N}$ & \pm 0.66 & $2.63^{\circ} \mathrm{N}$ & 2.29 & 1.57 \\
\hline & & $8.7-10$ & 9.4 & 58 & $0.30^{\circ} \mathrm{N}$ & \pm 1.39 & $1.15^{\circ} \mathrm{N}$ & 3.90 & 3.03 \\
\hline \multirow[t]{3}{*}{575} & $5.9^{\circ} \mathrm{N}, 135.0^{\circ} \mathrm{W}$ & $0-3.4$ & 1.7 & 125 & $4.13^{\circ} \mathrm{N}$ & \pm 0.68 & $5.05^{\circ} \mathrm{N}$ & 1.72 & 0.80 \\
\hline & & $5-6.4$ & 5.7 & 102 & $3.21^{\circ} \mathrm{N}$ & \pm 1.25 & $4.04^{\circ} \mathrm{N}$ & 2.64 & 1.81 \\
\hline & & $16-18$ & 17.0 & 50 & $0.06^{\circ} \mathrm{N}$ & \pm 1.67 & $1.70^{\circ} \mathrm{N}$ & 5.79 & 4.15 \\
\hline
\end{tabular}

\footnotetext{
age range used for tectonic calculations.

b Calculated by using the dipole formula and Kono's (1980) statistics.

c Calculated by using Kono's (1980) method.
}

d Calculated by using Epp's (1978) rotation poles for the Pacific Plate.

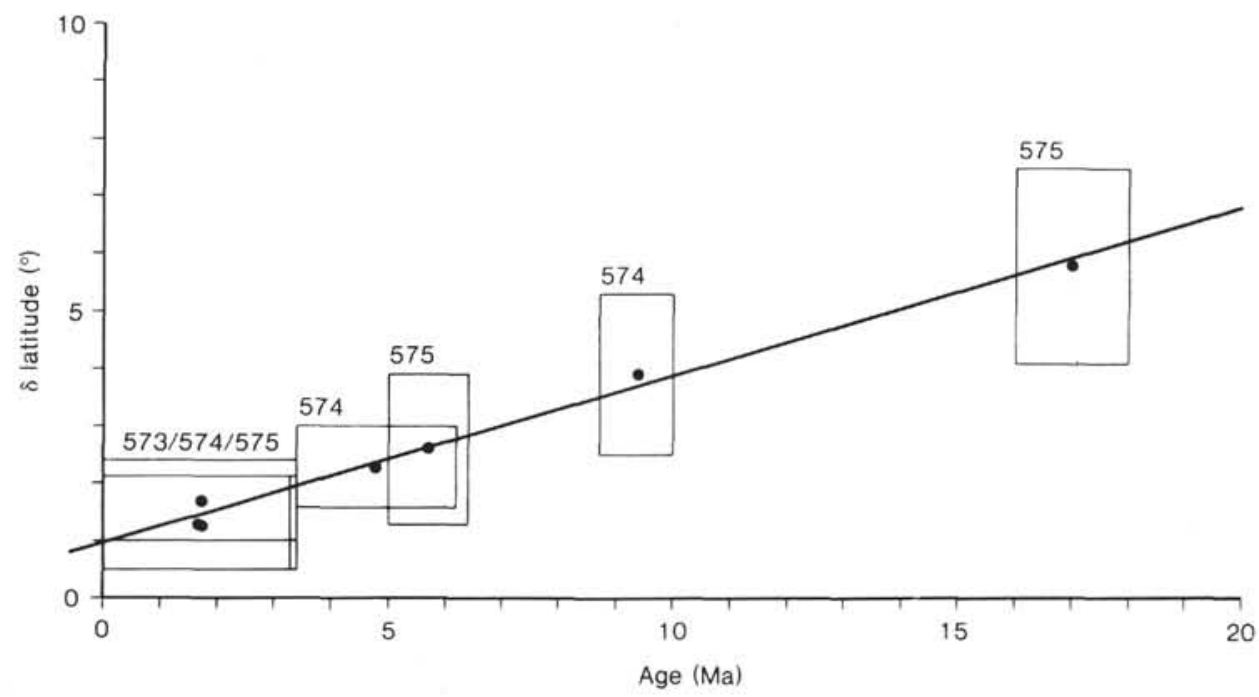

Figure 11. Delta latitude (site's present latitude minus its paleolatitude) versus age (Ma). Boxes represent the size of the error in latitude (which is based on Kono's [1980] statistics [Table 5]) and range of the error in age. The solid line is the regression curve (Table 5). 


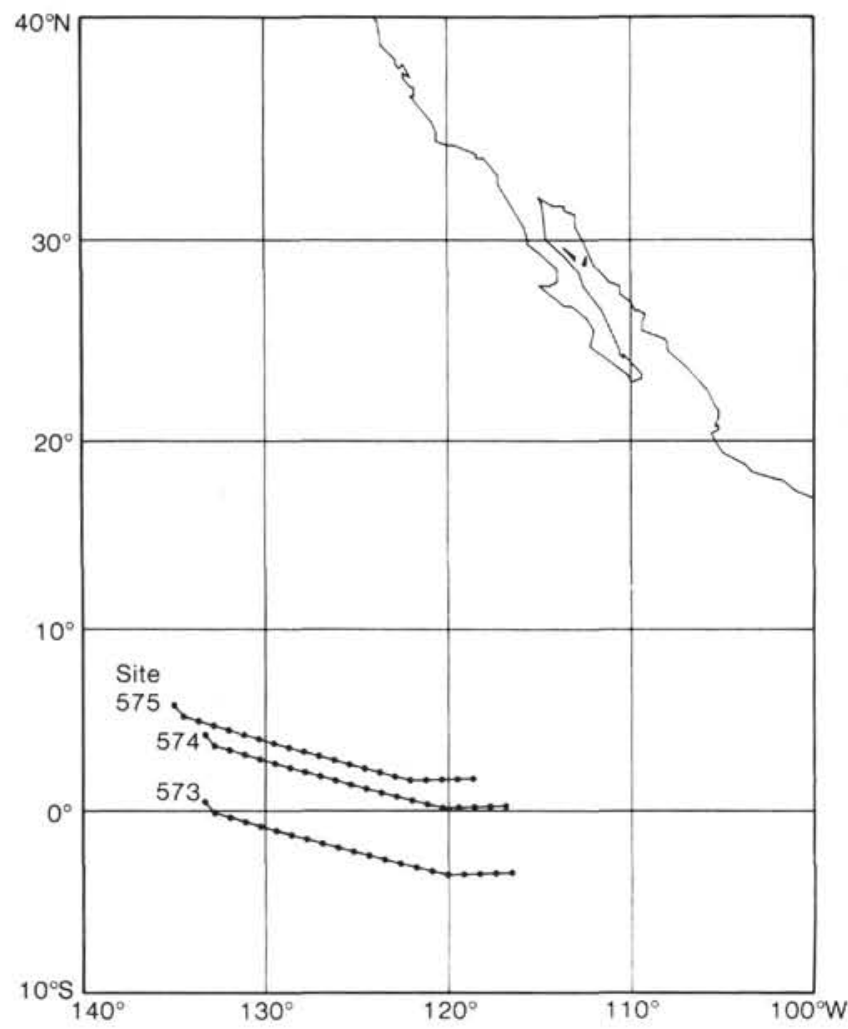

Figure 12. Stereographic projection of the migration of Sites 573, 574 and 575 . Site positions (in 1-m.y. steps) were calculated by using the rotation poles for the Pacific Plate that Epp (1978) derived from the movement of Pacific island chains.
Table 6. Pliocene to early Miocene paleo-pole positions for the Pacific Plate derived from Leg 85 sites and HIG piston cores, using the method of Gordon and Cox (1980).

\begin{tabular}{|c|c|c|c|c|c|c|}
\hline $\begin{array}{l}\text { Number of } \\
\text { DSDP site or } \\
\text { HIG piston core }\end{array}$ & $\begin{array}{c}\text { Present } \\
\text { longitude, } \\
\text { latitude }\end{array}$ & $\begin{array}{l}\text { Age range } \\
\text { used to } \\
\text { calculate } \\
\text { pole position } \\
\text { (Ma) }\end{array}$ & $\begin{array}{l}\text { Paleo-pole } \\
\text { position }\end{array}$ & $d p^{a}$ & $\mathrm{dm}^{\mathrm{a}}$ & $\begin{array}{c}\text { Number of } \\
\text { samples }\end{array}$ \\
\hline 573 & $0.5^{\circ} \mathrm{N}, 133.3^{\circ} \mathrm{W}$ & $0-3.3$ & & & & \\
\hline 574 & $4.2^{\circ} \mathrm{N}, 133.3^{\circ} \mathrm{W}$ & $0-6.2$ & & & & \\
\hline 575 & $5.9^{\circ} \mathrm{N}, 135.0^{\circ} \mathrm{W}$ & $0-6.4$ & $87.9^{\circ} \mathrm{N}, 59.6^{\circ} \mathrm{E}$ & $0.9^{\circ}$ & $3.3^{\circ}$ & 106 \\
\hline K 78.5-11 & $7.5^{\circ} \mathrm{N}, 169.7^{\circ} \mathrm{W}$ & $1.7-5.3$ & & & & \\
\hline K $76-1-07$ & $2.8^{\circ} \mathrm{S}, 177.9^{\circ} \mathrm{E}$ & $3-4.8$ & & & & \\
\hline 574 & $4.2^{\circ} \mathrm{N}, 133.3^{\circ} \mathrm{W}$ & $8.7-10$ & & & & \\
\hline K76.1-11 & $0.9^{\circ} \mathrm{N}, 178.6^{\circ} \mathrm{W}$ & $8-11$ & $85.1^{\circ} \mathrm{N}, 4.7^{\circ} \mathrm{E}$ & $2.3^{\circ}$ & $4.2^{\circ}$ & 71 \\
\hline K $76-1-17$ & $4.6^{\circ} \mathrm{N}, 176.6^{\circ} \mathrm{W}$ & $8-11.5$ & & & & \\
\hline 575 & $5.9^{\circ} \mathrm{N}, 135.0^{\circ} \mathrm{W}$ & $16-18$ & & & & \\
\hline K78-5-18 & $8.7^{\circ} \mathrm{N}, 166.9^{\circ} \mathrm{W}$ & $15-18$ & $79.5^{\circ} \mathrm{N}, 31.7^{\circ} \mathrm{W}$ & $2.9^{\circ}$ & $7.0^{\circ}$ & 35 \\
\hline $\mathrm{K} 72.36$ & $2.4^{\circ} \mathrm{S}, 177.8^{\circ} \mathrm{W}$ & $16-19$ & & & & \\
\hline
\end{tabular}

a Semi-axes of the $95 \%$ oval for the calculated pole position.

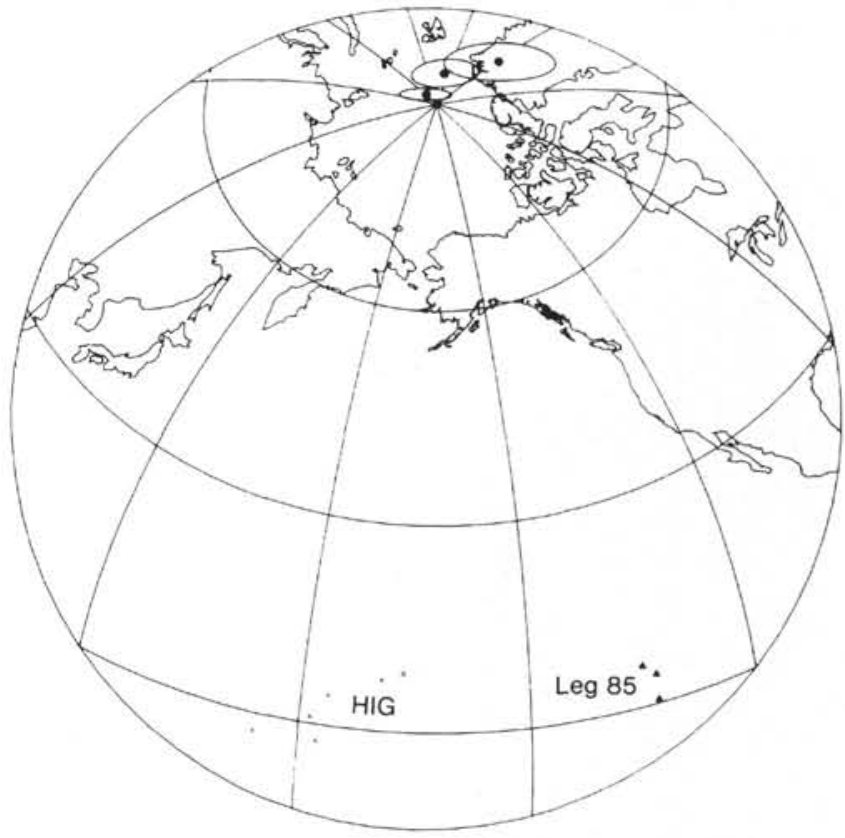

Figure 13. Apparent polar wander path determined on the basis of Leg 85 and HIG piston core paleomagnetic data. Poles and their $95 \%$ ovals are calculated for three time intervals ( 0 to $6 \mathrm{Ma}, 8$ to $11 \mathrm{Ma}, 16$ to $18 \mathrm{Ma}$ ) using the method of Gordon and Cox (1980). Crosses show HIG locations (Table 6), and triangles indicate Sites 573 to 575 . 
APPENDIX A

Paleomagnetic Data Derived from Late Miocene to Recent Leg 85 Samples (samples were measured at RUB)

\begin{tabular}{|c|c|c|c|c|c|c|c|c|}
\hline $\begin{array}{c}\text { Core-Section } \\
\text { (interval in cm) }\end{array}$ & $\begin{array}{l}\text { Sub-bottom } \\
\text { depth } \\
\text { (m) }\end{array}$ & $\underset{(\mathrm{G})}{J_{\mathrm{NRM}}}$ & $I_{\mathrm{NRM}}$ & $D_{\text {NRM }}$ & $I_{\text {stable }}$ & $D_{\text {stable }}$ & Polarity & $\begin{array}{l}\text { MDF } \\
(\mathrm{Oe})\end{array}$ \\
\hline \multicolumn{9}{|l|}{ Hole 573} \\
\hline $1-1,30-32$ & 0.31 & 3.83 E-6 & +12.0 & 343.1 & +12.1 & 343.7 & $N$ & 498 \\
\hline $1-1,70-72$ & 0.71 & $1.19 \mathrm{E}-6$ & +8.8 & 343.2 & +7.2 & 343.4 & $\mathrm{~N}$ & 388 \\
\hline $1-1,111-113$ & 1.12 & $1.20 \mathrm{E}-6$ & +0.0 & 17.3 & +1.5 & 16.2 & $\mathrm{~N}$ & 350 \\
\hline $1-2,20-22$ & 1.71 & $4.43 \mathrm{E}-6$ & +0.7 & 15.8 & +0.1 & 16.0 & $\mathrm{~N}$ & 281 \\
\hline $2-1,145-147$ & 3.46 & $2.35 \mathrm{E}-6$ & +0.3 & 282.2 & -2.3 & 283.8 & $\mathrm{~N}$ & 382 \\
\hline $2-2,38-40$ & 3.89 & $5.40 \mathrm{E}-6$ & +0.3 & 319.0 & +2.6 & 318.8 & $\mathrm{~N}$ & 347 \\
\hline $2-2,95-97$ & 4.46 & $4.12 \mathrm{E}-6$ & -7.3 & 338.7 & -5.6 & 337.7 & $\mathrm{~N}$ & 275 \\
\hline $2-2,145-147$ & 4.96 & $1.09 \mathrm{E}-6$ & -4.5 & 343.3 & -6.4 & 341.7 & $\mathrm{~N}$ & 411 \\
\hline $2-3,38-40$ & 5.39 & $1.26 \mathrm{E}-6$ & -9.4 & 357.3 & -11.4 & 359.0 & $\mathrm{~N}$ & 374 \\
\hline $2-3,95-97$ & 5.96 & $1.43 \mathrm{E}-6$ & +0.9 & 14.2 & +4.0 & 10.2 & $\mathrm{~N}$ & 308 \\
\hline $2-3,145-147$ & 6.46 & $7.78 \mathrm{E}-7$ & +0.8 & 12.5 & +11.6 & 29.7 & $\mathrm{~N}$ & 300 \\
\hline $2-4,36-38$ & 6.87 & $1.06 \mathrm{E}-6$ & -11.7 & 352.2 & -3.9 & 349.0 & $\mathrm{~N}$ & 325 \\
\hline $2-4,95-97$ & 7.46 & 2.75 E- 6 & -48.4 & 356.8 & -49.9 & 349.2 & $\mathrm{~N}$ & 265 \\
\hline $2-4,145-147$ & 7.96 & $4.14 \mathrm{E}-7$ & +7.7 & 322.7 & +10.6 & 318.7 & $\mathrm{~N}$ & 389 \\
\hline $2-5,36-38$ & 8.37 & $2.00 \mathrm{E}-7$ & +59.0 & 13.5 & +58.0 & 5.4 & $\mathrm{~N}$ & $>400$ \\
\hline $2-5,95-97$ & 8.96 & $5.32 \mathrm{E}-7$ & -3.3 & 351.7 & +12.7 & 359.2 & $\mathrm{~N}$ & 233 \\
\hline $2-5,143-145$ & 9.44 & $5.11 \mathrm{E}-7$ & -9.7 & 343.3 & +3.3 & 341.3 & $\mathrm{~N}$ & 399 \\
\hline $2-6,36-38$ & 7.87 & $1.45 \mathrm{E}-6$ & -5.8 & 319.8 & -5.1 & 319.6 & $\mathrm{~N}$ & 330 \\
\hline $2-6,95-97$ & 10.46 & $2.80 \mathrm{E}-6$ & -4.1 & 324.6 & -3.4 & 324.1 & $\mathrm{~N}$ & 310 \\
\hline $2-6,138-140$ & 10.89 & $1.56 \mathrm{E}-6$ & +0.9 & 314.7 & +3.1 & 314.8 & $\mathrm{~N}$ & 309 \\
\hline $2-7,36-38$ & 11.37 & $2.47 \mathrm{E}-6$ & -9.9 & 314.0 & -7.9 & 315.2 & $N$ & 345 \\
\hline $3-1,8-10$ & 11.59 & $8.23 \mathrm{E}-7$ & +3.6 & 308.0 & +7.4 & 307.2 & $N$ & 378 \\
\hline $3-1,68-70$ & 12.19 & $1.11 \mathrm{E}-6$ & -0.0 & 308.7 & -0.8 & 308.9 & $\mathrm{~N}$ & 371 \\
\hline $3-1,125-127$ & 12.76 & $4.28 \mathrm{E}-6$ & -2.1 & 355.3 & -2.5 & 354.6 & $\mathrm{~N}$ & 297 \\
\hline $3-1,146-148$ & 12.97 & 5.07 E-6 & +0.6 & 323.3 & -1.9 & 1.8 & $\mathrm{~N}$ & 176 \\
\hline $3-2,35-37$ & 13.36 & $9.23 \mathrm{E}-7$ & -20.6 & 189.8 & -5.7 & 185.9 & $\mathbf{R}$ & 559 \\
\hline $3-2,85-87$ & 13.86 & $1.02 \mathrm{E}-6$ & -13.6 & 193.6 & -12.5 & 193.4 & $\mathbf{R}$ & 499 \\
\hline $3-2,135-137$ & 14.36 & $4.77 \mathrm{E}-7$ & +3.6 & 129.3 & +52.3 & 189.0 & $\mathrm{R}$ & 466 \\
\hline $3-3,35-37$ & 14.86 & $5.04 \mathrm{E}-7$ & -7.3 & 196.8 & +0.2 & 189.4 & $\mathrm{R}$ & 390 \\
\hline $3-3,85-87$ & 15.36 & $9.97 \mathrm{E}-7$ & -4.6 & 213.1 & +5.6 & 202.7 & $\mathrm{R}$ & 463 \\
\hline $3-3,135-137$ & 15.86 & $1.06 \mathrm{E}-6$ & +13.7 & 212.1 & +32.4 & 212.1 & $\mathrm{R}$ & 472 \\
\hline $3-4,35-37$ & 16.36 & $5.89 \mathrm{E}-7$ & -30.5 & 265.4 & -20.6 & 211.0 & $\mathrm{R}$ & 368 \\
\hline $3-4,85-87$ & 16.86 & 8.03 E-7 & -29.3 & 271.8 & -24.2 & 264.4 & $\mathrm{~N}$ & 338 \\
\hline $3-4,135-137$ & 17.36 & 7.90 E-7 & -22.5 & 261.4 & -18.1 & 255.6 & $\mathrm{~N}$ & 365 \\
\hline $3-5,6-8$ & 17.57 & $4.06 \mathrm{E}-7$ & -14.4 & 303.5 & -19.9 & 221.0 & $\mathbf{R}$ & 198 \\
\hline $3-5,35-37$ & 17.86 & $3.20 \mathrm{E}-7$ & -20.6 & 209.1 & -12.8 & 191.2 & $\mathbf{R}$ & 397 \\
\hline $3-5,85-87$ & 18.36 & $9.21 \mathrm{E}-7$ & +22.1 & 154.7 & +5.5 & 128.6 & $\mathbf{R}$ & 478 \\
\hline $3-5,135-137$ & 18.86 & $1.82 \mathrm{E}-6$ & +2.5 & 131.9 & +5.0 & 133.0 & $\mathbf{R}$ & 399 \\
\hline $3-6,35-37$ & 19.36 & $2.51 \mathrm{E}-6$ & -0.4 & 128.9 & -0.2 & 130.5 & $\mathbf{R}$ & 347 \\
\hline $3-6,85-87$ & 19.86 & $4.30 \mathrm{E}-6$ & -9.2 & 138.2 & -3.1 & 138.0 & $\mathbf{R}$ & 361 \\
\hline $3-6,135-137$ & 20.36 & $1.25 \mathrm{E}-6$ & +6.4 & 148.0 & +12.6 & 144.1 & $\mathbf{R}$ & 433 \\
\hline $4-1,101-103$ & 22.02 & $2.72 \mathrm{E}-6$ & -7.4 & 265.9 & -7.1 & 265.2 & $\mathbf{R}$ & 420 \\
\hline $4-1,144-146$ & 22.45 & $5.00 \mathrm{E}-7$ & -32.9 & 242.2 & -12.4 & 248.4 & $\mathbf{R}$ & 572 \\
\hline $4-2,35-37$ & 22.86 & $1.71 \mathrm{E}-6$ & -2.0 & 234.8 & -1.9 & 234.0 & $\mathbf{R}$ & 386 \\
\hline $4-2,87-89$ & 23.38 & $1.11 \mathrm{E}-6$ & +6.1 & 225.9 & +8.1 & 225.2 & $\mathbf{R}$ & 408 \\
\hline $4-2,135-137$ & 23.86 & $3.19 \mathrm{E}-6$ & -2.1 & 213.5 & -3.4 & 208.7 & $\mathbf{R}$ & 388 \\
\hline $4-3,8-10$ & 24.09 & $2.05 \mathrm{E}-6$ & +6.8 & 204.4 & +6.0 & 200.5 & $\mathbf{R}$ & 364 \\
\hline $4-3,35-37$ & 24.36 & $1.25 \mathrm{E}-6$ & +13.3 & 194.0 & +8.4 & 191.6 & $\mathbf{R}$ & 398 \\
\hline $4-3,87-89$ & 24.88 & 5.77 E-7 & +1.2 & 179.3 & +4.0 & 184.7 & $\mathbf{R}$ & 454 \\
\hline $4-3,135-137$ & 25.36 & $2.61 \mathrm{E}-6$ & +0.5 & 359.5 & -4.1 & 7.5 & $\mathrm{~N}$ & 154 \\
\hline $4-4,35-37$ & 25.86 & $4.55 \mathrm{E}-6$ & +4.0 & 349.0 & +3.3 & 349.8 & $\mathrm{~N}$ & 260 \\
\hline $4-4,87-89$ & 26.38 & $2.25 \mathrm{E}-6$ & +6.2 & 349.6 & +4.6 & 347.5 & $\mathrm{~N}$ & 256 \\
\hline $4-4,135-137$ & 26.86 & $3.90 \mathrm{E}-6$ & -7.0 & 333.7 & -5.3 & 335.4 & $\mathrm{~N}$ & 248 \\
\hline $4-5,35-37$ & 27.36 & $1.98 \mathrm{E}-7$ & +20.3 & 184.4 & +5.0 & 161.2 & $\mathbf{R}$ & $>700$ \\
\hline $4-5,82-84$ & 27.83 & $1.13 \mathrm{E}-6$ & +13.4 & 176.7 & +5.8 & 148.6 & $\mathrm{R}$ & 456 \\
\hline $4-5,135-137$ & 28.36 & $1.01 \mathrm{E}-6$ & +1.2 & 153.3 & +7.2 & 155.9 & $\mathbf{R}$ & 384 \\
\hline $4-6,35-37$ & 28.86 & $2.30 \mathrm{E}-6$ & +1.6 & 144.4 & +5.2 & 143.4 & $\mathbf{R}$ & 441 \\
\hline $4-6,87-89$ & 29.38 & $1.70 \mathrm{E}-6$ & +11.7 & 142.8 & +0.8 & 141.9 & $\mathbf{R}$ & 432 \\
\hline $4-6,135-137$ & 29.86 & $1.21 \mathrm{E}-6$ & -6.8 & 134.3 & +2.9 & 135.8 & $\mathbf{R}$ & 473 \\
\hline $4-7,5-7$ & 30.06 & $1.61 \mathrm{E}-6$ & +2.3 & 132.3 & +5.5 & 135.4 & $\mathbf{R}$ & 369 \\
\hline $4-7,32-34$ & 30.33 & 9.87 E-7 & +17.4 & 141.9 & +13.6 & 143.3 & R & 426 \\
\hline $5-1,25-27$ & 30.76 & $9.86 \mathrm{E}-7$ & -17.9 & 18.5 & -16.7 & 19.6 & $\mathbf{R}$ & 449 \\
\hline $5-1,84-86$ & 31.35 & $1.46 \mathrm{E}-6$ & -1.3 & 49.3 & -0.7 & 51.5 & $\mathbf{R}$ & 393 \\
\hline $5-1,141-143$ & 31.92 & $1.23 \mathrm{E}-6$ & +1.9 & 81.5 & +3.9 & 80.8 & $\mathbf{R}$ & 403 \\
\hline $5-2,25-27$ & 32.26 & $1.11 \mathrm{E}-6$ & +15.2 & 94.3 & +4.2 & 100.3 & $\mathbf{R}$ & 487 \\
\hline $5-2,84-86$ & 32.85 & $1.18 \mathrm{E}-6$ & +23.8 & 116.0 & +10.4 & 122.2 & $\mathbf{R}$ & 537 \\
\hline $5-2,141-143$ & 33.42 & $4.73 \mathrm{E}-7$ & +7.0 & 144.6 & +5.4 & 144.8 & $\mathbf{R}$ & 422 \\
\hline $5-3,22-24$ & 33.73 & $1.09 \mathrm{E}-6$ & +4.7 & 148.3 & +2.4 & 148.0 & $\mathbf{R}$ & 415 \\
\hline
\end{tabular}


Appendix A. (Continued).

\begin{tabular}{|c|c|c|c|c|c|c|c|c|}
\hline $\begin{array}{l}\text { Core-Section } \\
\text { (interval in cm) }\end{array}$ & $\begin{array}{l}\text { Sub-bottom } \\
\text { depth } \\
\text { (m) }\end{array}$ & $\begin{array}{c}J_{\text {NRM }} \\
(\mathrm{G})\end{array}$ & $I_{\mathrm{NRM}}$ & $D_{\text {NRM }}$ & $I_{\text {stable }}$ & $D_{\text {stable }}$ & Polarity & $\begin{array}{l}\text { MDF } \\
(\mathrm{Oe})\end{array}$ \\
\hline \multicolumn{9}{|l|}{ Hole 573 (Cont.) } \\
\hline $5-3,93-95$ & 34.44 & $1.32 \mathrm{E}-6$ & +15.3 & 165.4 & +8.1 & 168.4 & $\mathbf{R}$ & 388 \\
\hline $5-3,137-139$ & 34.88 & $2.29 \mathrm{E}-6$ & -0.7 & 178.1 & -1.9 & 180.1 & $\mathbf{R}$ & 440 \\
\hline $5-4,31-33$ & 35.32 & $1.47 \mathrm{E}-6$ & +42.8 & 199.3 & +16.2 & 193.1 & $\mathbf{R}$ & 583 \\
\hline $5-4,85-87$ & 35.86 & $4.52 \mathrm{E}-6$ & +7.9 & 200.7 & +6.5 & 200.9 & $\mathbf{R}$ & 485 \\
\hline $5-4,127-129$ & 36.28 & $3.69 \mathrm{E}-6$ & -1.0 & 24.9 & -2.9 & 25.1 & $\mathrm{~N}$ & 471 \\
\hline $5-5,23-25$ & 36.74 & $2.33 \mathrm{E}-6$ & +4.5 & 23.8 & +0.2 & 26.8 & $\mathrm{~N}$ & 263 \\
\hline $5-5,85-87$ & 37.36 & 2.15 E-6 & -7.7 & 27.9 & -3.7 & 29.6 & $\mathrm{~N}$ & 218 \\
\hline $5-5,127-129$ & 37.78 & $4.09 \mathrm{E}-6$ & -2.6 & 30.9 & +0.4 & 33.1 & N & 260 \\
\hline $5-6,6-8$ & 38.07 & $7.06 \mathrm{E}-7$ & +9.2 & 19.3 & -6.3 & 30.3 & $\mathrm{~N}$ & 142 \\
\hline $5-6,32-34$ & 38.33 & $3.51 \mathrm{E}-6$ & -6.3 & 32.3 & -11.0 & 30.5 & N & 164 \\
\hline $5-6,85-87$ & 38.86 & $1.95 \mathrm{E}-6$ & -1.3 & 38.7 & -3.1 & 38.5 & $\mathrm{~N}$ & 191 \\
\hline $6-1,128-130$ & 40.59 & $2.12 \mathrm{E}-6$ & -0.7 & 14.7 & +3.0 & 16.6 & $\mathrm{~N}$ & 235 \\
\hline $6-2,22-24$ & 41.03 & 2.80 E-6 & -11.6 & 32.7 & -10.9 & 31.8 & $\mathrm{~N}$ & 226 \\
\hline $6-2,72-74$ & 41.53 & $2.65 \mathrm{E}-6$ & -2.7 & 19.4 & -3.7 & 17.2 & $\mathrm{~N}$ & 232 \\
\hline $6-2,122-124$ & 42.03 & $2.01 \mathrm{E}-6$ & -6.4 & 16.4 & -11.8 & 14.9 & $\mathrm{~N}$ & 201 \\
\hline $6-2,146-148$ & 42.27 & $7.91 \mathrm{E}-7$ & -1.2 & 203.6 & +3.7 & 197.8 & $\mathbf{R}$ & $>500$ \\
\hline $6-3,22-24$ & 42.53 & $2.12 \mathrm{E}-7$ & -46.6 & 63.6 & -1.6 & 190.4 & $\mathbf{R}$ & $>1000$ \\
\hline $6-3,83-85$ & 43.14 & $9.83 \mathrm{E}-7$ & +10.7 & 8.8 & +69.5 & 84.3 & $\mathbf{R}$ & 63 \\
\hline $6-3,105-107$ & 43.36 & $4.89 \mathrm{E}-6$ & -3.3 & 2.8 & -1.1 & 1.4 & $\mathrm{~N}$ & 268 \\
\hline $6-3,135-137$ & 43.66 & $3.36 \mathrm{E}-6$ & -0.3 & 7.1 & +6.2 & 9.0 & $N$ & 177 \\
\hline $6-4,22-24$ & 44.03 & $3.13 \mathrm{E}-6$ & -2.0 & 2.5 & -4.3 & 1.0 & $\mathrm{~N}$ & 208 \\
\hline $6-4,73-75$ & 44.54 & $9.15 \mathrm{E}-7$ & +31.3 & 174.1 & +14.3 & 180.5 & $\mathbf{R}$ & 391 \\
\hline $6-4,105-107$ & 44.86 & $6.15 \mathrm{E}-7$ & +32.1 & 175.5 & +15.0 & 179.1 & $\mathrm{R}$ & 494 \\
\hline $6-4,135-137$ & 45.16 & 2.63 E-6 & +6.7 & 177.7 & +3.3 & 178.8 & $\mathbf{R}$ & 384 \\
\hline $6-4,147-149$ & 45.28 & $3.46 \mathrm{E}-6$ & +0.9 & 173.7 & -0.3 & 171.2 & $\mathbf{R}$ & 352 \\
\hline $6-5,12-14$ & 45.43 & $4.32 \mathrm{E}-8$ & +46.0 & 134.4 & +46.2 & 113.9 & $\mathbf{R}$ & $>200$ \\
\hline $6-5,61-63$ & 45.92 & $6.56 \mathrm{E}-8$ & +7.5 & 350.7 & +32.4 & 356.9 & $\mathrm{~N}$ & $>200$ \\
\hline $6-5,114-116$ & 46.45 & $3.27 \mathrm{E}-7$ & +55.3 & 331.4 & +53.0 & 332.4 & $\mathrm{~N}$ & 223 \\
\hline $6-6,12-14$ & 46.93 & $2.18 \mathrm{E}-8$ & +18.8 & 12.4 & +24.9 & 2.1 & $\mathrm{~N}$ & 98 \\
\hline $6-6,57-59$ & 47.38 & $1.59 \mathrm{E}-7$ & -47.6 & 65.7 & -38.9 & 68.7 & $\mathrm{~N}$ & $>300$ \\
\hline $6-6,112-114$ & 47.93 & 1.77 E-7 & -41.5 & 40.8 & -38.8 & 38.2 & $\mathrm{~N}$ & 189 \\
\hline \multicolumn{9}{|l|}{ Hole 573A } \\
\hline $1-1,113-115$ & 5.64 & $1.82 \mathrm{E}-6$ & -6.2 & 203.5 & -5.8 & 8.3 & $\mathrm{~N}$ & 169 \\
\hline $1-1,138-140$ & 5.89 & $8.74 \mathrm{E}-7$ & +0.9 & 58.0 & -2.6 & 54.4 & $\mathrm{~N}$ & 408 \\
\hline $1-2,20-22$ & 6.21 & $1.56 \mathrm{E}-6$ & +9.1 & 36.7 & +10.2 & 33.9 & $\mathrm{~N}$ & 385 \\
\hline $1-2,94-96$ & 6.95 & $5.37 \mathrm{E}-6$ & -4.4 & 49.9 & -2.1 & 48.6 & $\mathrm{~N}$ & 332 \\
\hline $1-3,27-29$ & 7.78 & $3.95 \mathrm{E}-6$ & -3.3 & 50.3 & -3.7 & 49.9 & $\mathrm{~N}$ & 455 \\
\hline $1-3,90-92$ & 8.41 & 3.94 E-6 & +5.4 & 46.1 & +7.6 & 44.4 & $\mathrm{~N}$ & 270 \\
\hline $1-4,45-47$ & 9.46 & $6.09 \mathrm{E}-6$ & +2.3 & 28.8 & +1.2 & 27.1 & $\mathrm{~N}$ & 257 \\
\hline $1-4,105-107$ & 10.06 & $1.22 \mathrm{E}-6$ & +2.8 & 3.3 & +2.6 & 2.6 & $\mathrm{~N}$ & 302 \\
\hline $1-5,45-47$ & 10.96 & $4.02 \mathrm{E}-6$ & -1.1 & 350.4 & +0.9 & 350.8 & $\mathrm{~N}$ & 303 \\
\hline $1-5,105-107$ & 11.56 & $1.48 \mathrm{E}-6$ & +5.7 & 334.6 & +5.6 & 334.4 & $\mathrm{~N}$ & 373 \\
\hline $1-6,45-47$ & 12.46 & $1.64 \mathrm{E}-6$ & +8.2 & 309.1 & +8.7 & 309.1 & $\mathrm{~N}$ & 337 \\
\hline $1-6,105-107$ & 13.06 & $1.14 \mathrm{E}-6$ & +6.2 & 302.9 & -0.1 & 302.4 & $\mathrm{~N}$ & 358 \\
\hline $1-7,5-7$ & 13.56 & $5.30 \mathrm{E}-6$ & +2.9 & 300.6 & +3.5 & 301.2 & $\mathrm{~N}$ & 292 \\
\hline $1-7,29-31$ & 13.80 & $2.46 \mathrm{E}-6$ & +1.4 & 298.1 & -1.0 & 298.6 & $\mathrm{~N}$ & 241 \\
\hline $2-1,11-13$ & 14.12 & $1.03 \mathrm{E}-6$ & +4.1 & 354.7 & +12.9 & 353.9 & $\mathrm{~N}$ & 326 \\
\hline $2-1,37-39$ & 14.38 & $7.13 \mathrm{E}-7$ & +59.3 & 315.4 & +61.8 & 322.0 & $\mathrm{~N}$ & 396 \\
\hline $2-1,63-65$ & 14.64 & $1.03 \mathrm{E}-6$ & +81.8 & 245.3 & +80.2 & 270.1 & $\mathbf{R}$ & 371 \\
\hline $2-1,87-89$ & 14.88 & $6.17 \mathrm{E}-7$ & +32.3 & 158.4 & +19.2 & 161.8 & $\mathbf{R}$ & 567 \\
\hline $2-1,115-117$ & 15.16 & $3.28 \mathrm{E}-7$ & +23.2 & 152.6 & +0.3 & 180.3 & $\mathbf{R}$ & 747 \\
\hline $2-1,138-140$ & 15.39 & 2.32 E-6 & +4.1 & 182.9 & +4.1 & 183.7 & $\mathbf{R}$ & 654 \\
\hline $2-2,27-29$ & 15.78 & 2.30 E-7 & +76.9 & 318.5 & +13.7 & 200.4 & $\mathbf{R}$ & $>1000$ \\
\hline $2-2,47-49$ & 15.98 & $2.30 \mathrm{E}-6$ & +7.1 & 7.8 & +8.0 & 6.0 & $\mathrm{~N}$ & 169 \\
\hline $2-2,67-69$ & 16.18 & $1.95 \mathrm{E}-6$ & +6.5 & 14.6 & +9.3 & 15.2 & $\mathrm{~N}$ & 219 \\
\hline $2-2,112-114$ & 16.63 & 6.43 E-6 & +0.5 & 11.6 & +1.9 & 11.9 & $\mathrm{~N}$ & 276 \\
\hline $2-2,144-146$ & 16.95 & $1.50 \mathrm{E}-7$ & +45.6 & 192.5 & +16.0 & 197.7 & $\mathbf{R}$ & $>500$ \\
\hline $2-3,9-11$ & 17.10 & 2.99 E-7 & +29.9 & 230.3 & +10.9 & 199.5 & $\mathbf{R}$ & $>500$ \\
\hline $2-3,27-29$ & 17.28 & $1.62 \mathrm{E}-7$ & +35.8 & 33.2 & +11.2 & 192.6 & $\mathbf{R}$ & $>600$ \\
\hline $2-3,43-45$ & 17.44 & 2.74 E-7 & +46.9 & 34.6 & +8.3 & 191.2 & $\mathbf{R}$ & $>500$ \\
\hline $2-3,67-69$ & 17.68 & $9.23 \mathrm{E}-7$ & +21.3 & 353.3 & +7.6 & 189.5 & $\mathbf{R}$ & 658 \\
\hline $2-3,112-114$ & 18.13 & $1.54 \mathrm{E}-6$ & +3.2 & 4.9 & +62.9 & 215.8 & $\mathbf{R}$ & 86 \\
\hline $2-4,27-29$ & 18.78 & $2.49 \mathrm{E}-6$ & +2.7 & 186.0 & +2.9 & 186.7 & $\mathbf{R}$ & 384 \\
\hline $2-4,67-69$ & 19.18 & $2.46 \mathrm{E}-6$ & +1.4 & 178.3 & +2.4 & 181.2 & $\mathbf{R}$ & 360 \\
\hline $2-4,112-114$ & 19.63 & $4.09 \mathrm{E}-6$ & -4.2 & 183.5 & -0.7 & 182.6 & $\mathbf{R}$ & 398 \\
\hline $2-5,27-29$ & 20.28 & $8.83 \mathrm{E}-7$ & +9.9 & 176.3 & +8.8 & 178.8 & $\mathbf{R}$ & 365 \\
\hline $2-5,67-69$ & 20.68 & $1.15 \mathrm{E}-6$ & -8.4 & 178.8 & -4.0 & 177.7 & $R$ & 389 \\
\hline
\end{tabular}


Appendix A. (Continued).

\begin{tabular}{|c|c|c|c|c|c|c|c|c|}
\hline $\begin{array}{l}\text { Core-Section } \\
\text { (interval in cm) }\end{array}$ & $\begin{array}{l}\text { Sub-bottom } \\
\text { depth } \\
\text { (m) }\end{array}$ & $\begin{array}{l}J_{\text {NRM }} \\
(\mathrm{G})\end{array}$ & $I_{\mathrm{NRM}}$ & $D_{\mathrm{NRM}}$ & $I_{\text {stable }}$ & $D_{\text {stable }}$ & Polarity & $\begin{array}{l}\text { MDF } \\
(\mathrm{Oe})\end{array}$ \\
\hline \multicolumn{9}{|l|}{ Hole 573A (Cont.) } \\
\hline $2-5,112-114$ & 21.13 & $1.18 \mathrm{E}-6$ & +17.4 & 159.6 & +11.3 & 170.8 & R & 542 \\
\hline $2-6,25-27$ & 21.76 & $2.21 \mathrm{E}-6$ & +1.3 & 163.2 & +2.0 & 167.1 & R & 478 \\
\hline $3-1,53-55$ & 22.64 & 3.07 E-6 & -19.4 & 212.0 & -23.5 & 212.2 & $\mathbf{R}$ & 407 \\
\hline $3-1,88-90$ & 22.99 & 9.93 E-7 & +10.0 & 194.6 & +10.6 & 195.1 & $\mathrm{R}$ & 412 \\
\hline $3-1,135-137$ & 23.46 & $2.85 \mathrm{E}-7$ & -17.0 & 203.2 & +1.8 & 191.0 & $\mathbf{R}$ & 534 \\
\hline $3-2,17-19$ & 23.78 & $1.34 \mathrm{E}-6$ & -2.4 & 188.3 & +2.9 & 187.6 & $\mathrm{R}$ & 426 \\
\hline $3-2,117-119$ & 24.78 & $1.57 \mathrm{E}-6$ & +11.1 & 159.7 & +6.3 & 166.4 & $\mathbf{R}$ & 414 \\
\hline $3-3,16-18$ & 25.27 & $2.24 \mathrm{E}-7$ & -62.9 & 114.4 & -45.6 & 180.6 & $\mathbf{R}$ & 584 \\
\hline $3-3,36-38$ & 25.47 & $2.19 \mathrm{E}-6$ & +7.8 & 339.0 & +1.3 & 338.7 & $\mathrm{~N}$ & 79 \\
\hline $3-3,57-59$ & 25.68 & $2.87 \mathrm{E}-6$ & -15.5 & 338.3 & -14.1 & 340.8 & $\mathrm{~N}$ & 197 \\
\hline $3-3,116-118$ & 26.27 & $1.98 \mathrm{E}-6$ & -5.0 & 343.9 & -5.0 & 347.5 & $\mathrm{~N}$ & 187 \\
\hline $3-3,146-148$ & 26.57 & $3.59 \mathrm{E}-6$ & -12.1 & 347.3 & -13.0 & 346.1 & $\mathrm{~N}$ & 217 \\
\hline $3-4,11-13$ & 26.72 & $2.28 \mathrm{E}-6$ & +6.5 & 346.1 & +7.2 & 342.9 & $\mathrm{~N}$ & 64 \\
\hline $3-4,35-37$ & 26.96 & $7.99 \mathrm{E}-7$ & -55.0 & 71.6 & -19.4 & 160.3 & $\mathbf{R}$ & 452 \\
\hline $3-4,71-73$ & 27.32 & $1.98 \mathrm{E}-6$ & +3.5 & 182.7 & -1.0 & 174.2 & $\mathrm{R}$ & 383 \\
\hline $3-4,103-105$ & 27.64 & $9.47 \mathrm{E}-7$ & +19.3 & 156.9 & +7.2 & 164.1 & $\mathrm{R}$ & 365 \\
\hline $3-4,138-140$ & 27.99 & $1.51 \mathrm{E}-6$ & +10.6 & 180.1 & +5.7 & 172.9 & $\mathrm{R}$ & 352 \\
\hline $3-5,35-37$ & 28.46 & $2.01 \mathrm{E}-6$ & +12.2 & 176.6 & +5.5 & 171.0 & $\mathrm{R}$ & 438 \\
\hline $3-5,71-73$ & 28.82 & $2.01 \mathrm{E}-6$ & -8.3 & 161.6 & -6.9 & 169.5 & $\mathrm{R}$ & 416 \\
\hline $3-5,103-105$ & 29.14 & $8.40 \mathrm{E}-7$ & -60.5 & 102.8 & -16.1 & 177.8 & $\mathrm{R}$ & 494 \\
\hline $3-5,137-139$ & 29.48 & $1.17 \mathrm{E}-6$ & -16.8 & 185.5 & -3.8 & 182.2 & $\mathrm{R}$ & 444 \\
\hline $3-6,35-37$ & 29.96 & $1.22 \mathrm{E}-6$ & -8.5 & 206.8 & +4.8 & 199.0 & $\mathrm{R}$ & 366 \\
\hline $3-6,71-73$ & 30.32 & $1.71 \mathrm{E}-6$ & +4.1 & 191.7 & -0.2 & 199.1 & $\mathrm{R}$ & 478 \\
\hline $3-6,113-115$ & 30.74 & $8.73 \mathrm{E}-7$ & -11.3 & 217.4 & -20.9 & 203.8 & R & 399 \\
\hline $4-1,83-85$ & 32.14 & $3.23 \mathrm{E}-7$ & -20.7 & 217.7 & -10.9 & 219.3 & $\mathrm{R}$ & 451 \\
\hline $4-1,131-133$ & 32.62 & $3.96 \mathrm{E}-7$ & -3.7 & 15.8 & +10.1 & 187.4 & $\mathbf{R}$ & 71 \\
\hline $4-2,5-7$ & 32.86 & $3.20 \mathrm{E}-7$ & +0.9 & 207.8 & +1.7 & 197.9 & $\mathrm{R}$ & 536 \\
\hline $4-2,32-34$ & 33.13 & $6.14 \mathrm{E}-7$ & +7.6 & 198.3 & +3.2 & 200.1 & $\mathrm{R}$ & 440 \\
\hline $4-2,83-85$ & 33.64 & $5.71 \mathrm{E}-7$ & +14.6 & 183.4 & +14.5 & 185.9 & $\mathrm{R}$ & 387 \\
\hline $4-2,131-133$ & 34.12 & $2.24 \mathrm{E}-6$ & +11.6 & 180.6 & +8.4 & 182.2 & $\mathrm{R}$ & 473 \\
\hline $4-3,5-7$ & 34.36 & $2.43 \mathrm{E}-6$ & +6.0 & 177.7 & +1.2 & 180.6 & R & 490 \\
\hline $4-3,3 i-34$ & 34.63 & $1.05 \mathrm{E}-6$ & +6.3 & 166.3 & +7.8 & 168.1 & $R$ & 554 \\
\hline $4-3,57-59$ & 34.88 & $3.49 \mathrm{E}-6$ & +2.3 & 168.5 & +1.6 & 167.7 & $\mathrm{R}$ & 471 \\
\hline $4-3,83-85$ & 35.14 & $2.99 \mathrm{E}-6$ & -4.5 & 348.6 & -8.4 & 350.8 & $\mathrm{~N}$ & 275 \\
\hline $4-3,131-133$ & 35.62 & $2.40 \mathrm{E}-6$ & +1.2 & 339.3 & +0.1 & 345.3 & $\mathrm{~N}$ & 238 \\
\hline $4-4,32-34$ & 36.13 & $1.43 \mathrm{E}-6$ & -3.7 & 0.8 & -2.5 & 355.4 & $\mathrm{~N}$ & 188 \\
\hline $4-4,83-85$ & 36.64 & 8.16 E-7 & +7.8 & 341.0 & +0.8 & 352.2 & $N$ & 93 \\
\hline $4-4,131-133$ & 37.12 & 2.39 E-6 & -0.5 & 354.2 & +0.9 & 353.2 & $\mathrm{~N}$ & 308 \\
\hline $4-5,32-34$ & 37.63 & 1.73 E-6 & +4.3 & 348.8 & -1.2 & 349.7 & $\mathrm{~N}$ & 175 \\
\hline $4-5,83-85$ & 38.14 & $2.32 \mathrm{E}-6$ & +10.2 & 350.4 & +4.0 & 357.0 & $\mathrm{~N}$ & 185 \\
\hline $4-5,131-133$ & 38.62 & 2.71 E-6 & -9.1 & 355.0 & +1.2 & 351.4 & $\mathrm{~N}$ & 232 \\
\hline $4-6,32-34$ & 39.13 & 2.76 E-6 & -3.1 & 354.2 & +3.5 & 349.1 & $\mathrm{~N}$ & 250 \\
\hline $4-6,83-85$ & 39.64 & $3.07 \mathrm{E}-6$ & +6.1 & 349.2 & +11.1 & 353.2 & $\mathrm{~N}$ & 242 \\
\hline $5-1,98-100$ & 40.79 & $4.30 \mathrm{E}-6$ & -17.1 & 14.4 & -18.1 & 11.6 & $\mathrm{~N}$ & 232 \\
\hline $5-1,130-132$ & 41.11 & $1.31 \mathrm{E}-6$ & +20.5 & 211.1 & +7.9 & 201.9 & $\mathbf{R}$ & 551 \\
\hline $5-2,25-27$ & 41.56 & $1.21 \mathrm{E}-6$ & +5.4 & 174.0 & +1.4 & 181.3 & R & 421 \\
\hline $5-2,68-70$ & 41.99 & $7.47 \mathrm{E}-7$ & -3.5 & 8.0 & +12.9 & 187.2 & $\mathrm{R}$ & 32 \\
\hline $5-2,113-115$ & 42.44 & 3.87 E-6 & -1.7 & 6.0 & -2.6 & 3.0 & $\mathrm{~N}$ & 200 \\
\hline $5-2,146-148$ & 42.77 & $6.50 \mathrm{E}-6$ & +2.8 & 354.8 & +3.8 & 356.8 & $\mathrm{~N}$ & 267 \\
\hline $5-3,25-27$ & 43.06 & $1.15 \mathrm{E}-6$ & -1.3 & 9.2 & +20.5 & 4.7 & $\mathrm{~N}$ & 40 \\
\hline $5-3,68-70$ & 43.49 & $1.38 \mathrm{E}-6$ & +13.4 & 160.1 & +14.1 & 168.8 & $\mathrm{R}$ & 363 \\
\hline $5-3,113-115$ & 43.94 & $2.71 \mathrm{E}-6$ & -6.0 & 181.3 & -1.9 & 179.5 & $\mathrm{R}$ & 447 \\
\hline $5-3,136-138$ & 44.17 & $3.31 \mathrm{E}-6$ & +6.0 & 181.3 & +3.3 & 179.9 & $\mathrm{R}$ & 383 \\
\hline $5-3,143-145$ & 44.24 & $3.51 \mathrm{E}-6$ & +17.0 & 171.6 & +15.2 & 176.8 & $\mathrm{R}$ & 439 \\
\hline $5-4,45-47$ & 44.76 & $7.14 \mathrm{E}-8$ & +14.8 & 2.4 & no & able & & 399 \\
\hline $5-4,131-133$ & 45.62 & $6.23 \mathrm{E}-8$ & -15.1 & 6.5 & no & able & & 277 \\
\hline $5-5,51-53$ & 46.32 & $3.96 \mathrm{E}-8$ & +58.7 & 283.8 & no & able & & \\
\hline $5-5,115-117$ & 46.96 & 2.60 E-8 & +74.3 & 345.5 & no & able & & \\
\hline $5-6,8-10$ & 47.39 & $1.04 \mathrm{E}-7$ & +35.3 & 1.4 & no: & able & & \\
\hline $5-6,90-92$ & 48.21 & $6.26 \mathrm{E}-8$ & +8.9 & 118.5 & no & able & & \\
\hline $6-1,65-67$ & 49.86 & $4.45 \mathrm{E}-7$ & -8.8 & 177.2 & no & able & & 105 \\
\hline $6-2,61-63$ & 51.32 & $9.76 \mathrm{E}-8$ & +71.4 & 254.1 & no & able & & 31 \\
\hline $6-3,81-83$ & 53.02 & $3.52 \mathrm{E}-8$ & +57.0 & 289.6 & nos & able & & \\
\hline $6-4,58-60$ & 54.29 & $2.96 \mathrm{E}-8$ & -42.6 & 294.0 & nos & able & & \\
\hline $6-5,90-92$ & 56.11 & $1.05 \mathrm{E}-7$ & +82.6 & 336.5 & nos & able & & \\
\hline $6-6,18-20$ & 56.89 & $2.15 \mathrm{E}-8$ & +15.8 & 323.0 & nos & able & & \\
\hline \multicolumn{9}{|l|}{ Hole 574} \\
\hline $1-1,79-81$ & 0.80 & $4.54 \mathrm{E}-6$ & -1.5 & 16.2 & +0.9 & 14.0 & $\mathrm{~N}$ & 250 \\
\hline $1-1,129-131$ & 1.30 & $3.37 \mathrm{E}-6$ & +6.2 & 16.0 & +5.5 & 13.9 & $\mathrm{~N}$ & 201 \\
\hline
\end{tabular}


Appendix A. (Continued).

\begin{tabular}{|c|c|c|c|c|c|c|c|c|}
\hline $\begin{array}{l}\text { Core-Section } \\
\text { (interval in } \mathrm{cm} \text { ) }\end{array}$ & $\begin{array}{l}\text { Sub-bottom } \\
\text { depth } \\
\text { (m) }\end{array}$ & $\underset{(\mathrm{G})}{J_{\text {NRM }}}$ & $I_{\mathrm{NRM}}$ & $D_{\text {NRM }}$ & $I_{\text {stable }}$ & $D_{\text {stable }}$ & Polarity & $\begin{array}{l}\text { MDF } \\
(\mathrm{Oe})\end{array}$ \\
\hline \multicolumn{9}{|l|}{ Hole 574 (Cont.) } \\
\hline $1-2,29-31$ & 1.80 & 5.51 E-6 & +12.0 & 5.5 & +12.4 & 1.6 & $\mathrm{~N}$ & 156 \\
\hline $1-2,54-56$ & 2.05 & $4.39 \mathrm{E}-6$ & +11.1 & 4.2 & +6.8 & 1.0 & $\mathrm{~N}$ & 147 \\
\hline $1-2,104-106$ & 2.55 & $4.72 \mathrm{E}-6$ & +2.8 & 0.7 & -0.6 & 356.7 & $\mathrm{~N}$ & 196 \\
\hline $1-2,129-131$ & 2.80 & 5.75 E-6 & +15.0 & 336.5 & +13.6 & 348.9 & $\mathrm{~N}$ & 225 \\
\hline $1-3,4-6$ & 3.05 & 3.10 E-6 & $\begin{array}{r}+4.9 \\
\end{array}$ & 12.1 & $\begin{array}{r}+5.6 \\
\end{array}$ & 11.3 & $\mathrm{~N}$ & 198 \\
\hline $1-3,29-31$ & 3.30 & $6.61 \mathrm{E}-6$ & +0.1 & 355.5 & +5.5 & 353.8 & $\mathrm{~N}$ & 216 \\
\hline $1-3,54-56$ & 3.55 & $4.20 \mathrm{E}-6$ & +24.4 & 351.5 & +9.5 & 352.9 & $\mathrm{~N}$ & 95 \\
\hline $1-3,79-81$ & 3.80 & 4.21 E-6 & +7.1 & 355.3 & +6.0 & 347.2 & $\mathrm{~N}$ & 131 \\
\hline $1-3,104-106$ & 4.05 & 1.14 E-6 & -7.2 & 38.5 & +10.6 & 351.5 & $\mathrm{~N}$ & 464 \\
\hline $1-3,129-131$ & 4.30 & $1.78 \mathrm{E}-6$ & +7.2 & 345.9 & +8.9 & 350.8 & $\mathrm{~N}$ & 280 \\
\hline $2-1,14-16$ & 4.65 & 2.93 E-6 & -68.5 & 242.7 & -79.5 & 231.4 & $\mathbf{R}$ & 290 \\
\hline $2-1,37-39$ & 4.88 & 2.27 E-7 & -63.2 & 277.8 & +28.9 & 261.0 & $\mathbf{R}$ & 19 \\
\hline $2-1,67-69$ & 5.18 & $2.05 \mathrm{E}-6$ & +18.9 & 202.1 & -28.9 & 164.1 & $\mathbf{R}$ & 285 \\
\hline $2-1,94-96$ & 5.45 & 2.34 E-6 & +2.9 & 149.0 & -0.1 & 151.3 & $\mathbf{R}$ & 418 \\
\hline $2-1,119-121$ & 5.70 & 2.73 E-6 & -3.8 & 157.4 & -3.3 & 161.0 & $\mathrm{R}$ & 508 \\
\hline $2-2,4-6$ & 6.05 & $1.13 \mathrm{E}-6$ & -8.4 & 328.9 & -2.2 & 167.5 & $\mathrm{R}$ & 510 \\
\hline $2-2,29-31$ & 6.30 & 2.06 E-6 & -5.6 & 165.5 & -6.1 & 165.9 & $\mathrm{R}$ & 388 \\
\hline $2-2,54-56$ & 6.55 & 3.37 E-6 & +3.4 & 176.5 & -2.4 & 172.3 & $\mathrm{R}$ & 434 \\
\hline $2-2,79-81$ & 6.80 & $1.58 \mathrm{E}-6$ & +4.0 & 152.5 & -1.3 & 166.0 & $\mathbf{R}$ & 468 \\
\hline $2-2,104-106$ & 7.05 & $1.53 \mathrm{E}-6$ & -1.0 & 172.0 & -2.5 & 171.9 & $\mathrm{R}$ & 429 \\
\hline $2-2,129-131$ & 7.30 & 5.01 E-7 & +2.8 & 163.3 & +8.7 & 179.7 & $\mathrm{R}$ & 521 \\
\hline $2-3,4-6$ & 7.55 & $1.28 \mathrm{E}-6$ & -0.2 & 178.1 & -7.9 & 179.0 & $\mathbf{R}$ & 442 \\
\hline $2-3,29-31$ & 7.80 & $1.60 \mathrm{E}-6$ & -14.3 & 164.7 & -12.4 & 174.2 & $\mathbf{R}$ & 421 \\
\hline $2-3,54-56$ & 8.05 & 3.84 E-6 & 19.7 & 9.6 & +5.8 & 10.7 & $\mathrm{~N}$ & 121 \\
\hline $2-3,79-81$ & 8.30 & $5.66 \mathrm{E}-6$ & +9.7 & 10.3 & +9.1 & 11.1 & $\mathrm{~N}$ & 161 \\
\hline $2-3,104-106$ & 8.55 & 5.73 E-6 & +10.4 & 3.9 & +12.6 & 4.9 & $\mathrm{~N}$ & 173 \\
\hline $2-3,129-131$ & 8.80 & 5.68 E-6 & $\begin{array}{r}+5.4 \\
\end{array}$ & 8.0 & $\begin{array}{r}+8.4 \\
\end{array}$ & 12.3 & $\mathrm{~N}$ & 171 \\
\hline $2-4,4-6$ & 9.05 & 9.31 E-7 & -12.4 & 184.2 & -5.7 & 185.6 & $\mathrm{R}$ & 467 \\
\hline $2-4,29-31$ & 9.30 & 8.64 E-7 & +0.3 & 193.7 & -1.6 & 181.3 & $\mathrm{R}$ & 456 \\
\hline $2-4,54-56$ & 9.55 & $2.19 \mathrm{E}-6$ & +3.2 & 195.1 & -4.9 & 185.2 & $\mathrm{R}$ & 398 \\
\hline $2-4,79-81$ & 9.80 & $4.39 \mathrm{E}-7$ & -29.7 & 354.7 & -6.6 & 183.5 & $\mathrm{R}$ & 552 \\
\hline $2-4,104-106$ & 10.05 & $1.57 \mathrm{E}-6$ & -10.7 & 185.5 & -9.8 & 185.2 & $\mathrm{R}$ & 416 \\
\hline $2-4,129-131$ & 10.30 & $1.91 \mathrm{E}-6$ & -31.5 & 186.4 & -15.8 & 184.3 & $\ddot{R}$ & 392 \\
\hline $2-5,4-6$ & 10.55 & $1.16 \mathrm{E}-6$ & +5.8 & 182.0 & -6.8 & 184.1 & $\mathbf{R}$ & 440 \\
\hline $2-5,29-31$ & 10.80 & $3.12 \mathrm{E}-7$ & +9.3 & 168.8 & -8.6 & 180.1 & $\mathbf{R}$ & 560 \\
\hline $2-5,54-56$ & 11.05 & 5.70 E-7 & +20.6 & 214.9 & +8.3 & 179.0 & $\mathbf{R}$ & 599 \\
\hline $2-5,79-81$ & 11.30 & $5.49 \mathrm{E}-7$ & -4.5 & 177.8 & -9.6 & 178.0 & $\mathbf{R}$ & 493 \\
\hline $2-5,104-106$ & 11.55 & $2.64 \mathrm{E}-6$ & +0.8 & 152.6 & +1.5 & 161.1 & $\mathrm{R}$ & 431 \\
\hline $2-5,129-131$ & 11.80 & $6.73 \mathrm{E}-7$ & +14.5 & 5.8 & +45.5 & 38.7 & $\mathrm{~N}$ & 49 \\
\hline $2-6,4-6$ & 12.05 & $2.05 \mathrm{E}-6$ & +11.3 & 1.2 & +8.8 & 14.0 & $\mathrm{~N}$ & 144 \\
\hline $2-6,29-31$ & 12.30 & $2.09 \mathrm{E}-6$ & +14.0 & 10.9 & +14.3 & 13.0 & $\mathrm{~N}$ & 186 \\
\hline $2-6,54-56$ & 12.55 & 2.61 E-6 & +3.6 & 357.9 & +16.7 & 3.6 & $\mathrm{~N}$ & 74 \\
\hline $2-6,79-81$ & 12.80 & 4.06 E-6 & +8.1 & 9.2 & +3.1 & 13.2 & $\mathrm{~N}$ & 75 \\
\hline $2-6,104-106$ & 13.05 & $5.25 \mathrm{E}-6$ & +10.8 & 359.2 & +13.8 & 8.1 & $\mathrm{~N}$ & 135 \\
\hline $2-6,129-131$ & 13.30 & 7.02 E-6 & +5.2 & 8.7 & +4.6 & 11.0 & $\mathrm{~N}$ & 159 \\
\hline $2-7,4-6$ & 13.55 & $6.30 \mathrm{E}-6$ & +5.4 & 18.1 & +6.4 & 23.1 & $\mathrm{~N}$ & 103 \\
\hline $2-7,29-31$ & 13.80 & 5.80 E-6 & +11.1 & 15.9 & +15.7 & 17.4 & $\mathrm{~N}$ & 95 \\
\hline $3-1,75-77$ & 14.76 & 9.45 E-6 & -86.2 & 249.2 & -85.0 & 217.7 & $\mathbf{R}$ & 274 \\
\hline $3-1,97-99$ & 14.98 & $5.15 \mathrm{E}-7$ & +11.2 & 321.5 & -29.5 & 254.8 & $\mathbf{R}$ & 348 \\
\hline $3-1,118-120$ & 15.19 & 1.74 E-6 & -39.0 & 330.4 & -41.8 & 295.1 & $\mathbf{R}$ & 217 \\
\hline $3-2,16-18$ & 15.67 & $1.83 \mathrm{E}-6$ & -17.8 & 126.3 & -6.1 & 137.2 & $\mathrm{R}$ & 388 \\
\hline $3-2,87-89$ & 16.38 & 2.81 E-6 & -80.5 & 130.1 & -78.2 & 125.5 & $\mathbf{R}$ & 321 \\
\hline $3-2,136-138$ & 16.87 & $1.25 \mathrm{E}-6$ & -61.8 & 65.3 & -30.0 & 60.4 & $\mathrm{~N}$ & 315 \\
\hline $3-3,15-17$ & 17.16 & $1.67 \mathrm{E}-6$ & +14.6 & 332.3 & +6.2 & 305.9 & $\mathrm{~N}$ & 88 \\
\hline $3-3,39-41$ & 17.40 & 2.49 E-6 & +11.6 & 352.8 & +9.8 & 351.8 & $\mathrm{~N}$ & 208 \\
\hline $3-3,112-114$ & 18.13 & 3.34 E-6 & -66.3 & 335.4 & -68.8 & 346.5 & $\mathrm{~N}$ & 300 \\
\hline $3-3,136-138$ & 18.37 & 1.06 E-6 & -46.2 & 231.8 & -49.5 & 210.9 & $\mathbf{R}$ & 299 \\
\hline $3-4,8-10$ & 18.59 & $2.20 \mathrm{E}-6$ & -67.4 & 115.9 & -63.7 & 136.2 & $\mathbf{R}$ & 284 \\
\hline $3-4,29-31$ & 18.80 & $6.45 \mathrm{E}-7$ & -18.0 & 147.6 & -4.6 & 148.5 & $\mathbf{R}$ & 449 \\
\hline $3-4,54-56$ & 19.05 & $1.52 \mathrm{E}-6$ & +2.5 & 146.0 & +5.3 & 152.3 & $\mathbf{R}$ & 462 \\
\hline $3-4,79-81$ & 19.30 & 1.16 E-6 & +0.7 & 153.9 & +3.7 & 160.4 & $\mathrm{R}$ & 418 \\
\hline $3-4,104-106$ & 19.55 & $2.62 \mathrm{E}-7$ & +79.6 & 239.4 & +4.6 & 162.6 & $\mathbf{R}$ & $>1000$ \\
\hline $3-4,129-131$ & 19.80 & $9.06 \mathrm{E}-7$ & -5.5 & 159.9 & -2.2 & 164.0 & $\mathbf{R}$ & 498 \\
\hline $3-5,4-6$ & 20.05 & $1.13 \mathrm{E}-6$ & -3.0 & 144.1 & -1.5 & 165.3 & $\mathbf{R}$ & 443 \\
\hline $3-5,29-31$ & 20.30 & 2.41 E-6 & +8.2 & 179.6 & +2.7 & 180.4 & $\mathbf{R}$ & 424 \\
\hline $3-5,54-56$ & 20.55 & $1.54 \mathrm{E}-6$ & 1.3 & 171.5 & +0.1 & 177.6 & $\mathbf{R}$ & 384 \\
\hline $3-5,75-77$ & 20.76 & 1.99 E-6 & +2.9 & 181.4 & -0.9 & 181.0 & $\mathbf{R}$ & 390 \\
\hline $3-5,104-106$ & 21.05 & 1.87 E-6 & +0.7 & 179.2 & -4.9 & 178.0 & $\mathbf{R}$ & 396 \\
\hline $3-5,129-131$ & 21.30 & $6.86 \mathrm{E}-7$ & +8.4 & 133.4 & -3.1 & 179.0 & $\mathbf{R}$ & 495 \\
\hline $3-6,4-6$ & 21.55 & 1.51 E-6 & +5.3 & 174.6 & -0.6 & 189.5 & $\mathbf{R}$ & 437 \\
\hline $3-6,29-31$ & 21.80 & 1.67 E-6 & -12.3 & 186.2 & -8.7 & 191.0 & R & 449 \\
\hline $3-6,54-56$ & 22.05 & 1.42 E-6 & -4.5 & 193.1 & -5.3 & 197.4 & $\mathbf{R}$ & 401 \\
\hline $3-6,79-81$ & 22.30 & $2.39 \mathrm{E}-6$ & -13.6 & 180.0 & -8.9 & 190.3 & $\mathrm{R}$ & 424 \\
\hline
\end{tabular}


Appendix A. (Continued).

\begin{tabular}{|c|c|c|c|c|c|c|c|c|}
\hline $\begin{array}{c}\text { Core-Section } \\
\text { (interval in cm) }\end{array}$ & $\begin{array}{l}\text { Sub-bottom } \\
\text { depth } \\
\text { (m) }\end{array}$ & $\begin{array}{c}J_{\text {NRM }} \\
(\mathrm{G})\end{array}$ & $I_{\mathrm{NRM}}$ & $D_{\mathrm{NRM}}$ & $I_{\text {stable }}$ & $D_{\text {stable }}$ & Polarity & $\begin{array}{l}\text { MDF } \\
\text { (Oe) }\end{array}$ \\
\hline \multicolumn{9}{|l|}{ Hole 574 (Cont.) } \\
\hline $3-6,104-106$ & 22.55 & $1.17 \mathrm{E}-6$ & -5.7 & 193.5 & +0.8 & 202.2 & $\mathbf{R}$ & 500 \\
\hline $3-6,129-131$ & 22.80 & $1.06 \mathrm{E}-6$ & +57.8 & 86.5 & +6.3 & 207.7 & $\mathrm{R}$ & 487 \\
\hline $3-7,4-6$ & 23.05 & $7.27 \mathrm{E}-7$ & -44.5 & 193.2 & -4.8 & 202.9 & $\mathrm{R}$ & 532 \\
\hline $3-7,29-31$ & 23.30 & $4.60 \mathrm{E}-7$ & +4.3 & 105.3 & -10.0 & 204.2 & $\mathbf{R}$ & 694 \\
\hline $4-1,12-14$ & 23.63 & 6.45 E-6 & -80.7 & 40.9 & -75.0 & 62.9 & $\mathrm{~N}$ & 307 \\
\hline $4-1,50-52$ & 24.01 & $7.09 \mathrm{E}-7$ & -1.3 & 50.2 & -18.8 & 35.3 & $\mathrm{~N}$ & 267 \\
\hline $4-1,91-93$ & 24.42 & 2.76 E-7 & -2.4 & 13.5 & -3.0 & 7.8 & $\mathrm{~N}$ & 287 \\
\hline $4-1,123-125$ & 24.74 & 9.82 E-7 & +18.5 & 18.9 & +10.1 & 27.2 & $\mathrm{~N}$ & 273 \\
\hline $4-2,15-17$ & 25.16 & $2.36 \mathrm{E}-6$ & +13.5 & 17.1 & +1.8 & 28.5 & $\mathrm{~N}$ & 142 \\
\hline $4-2,48-50$ & 25.49 & $1.29 \mathrm{E}-6$ & +21.0 & 15.2 & +17.5 & 17.2 & $\mathrm{~N}$ & 176 \\
\hline $4-2,85-87$ & 25.86 & 2.37 E-6 & +64.3 & 345.8 & +44.0 & 13.0 & $\mathrm{~N}$ & 304 \\
\hline $4-2,102-104$ & 26.03 & 8.50 E-7 & -31.9 & 320.1 & -22.3 & 133.9 & $\mathbf{R}$ & 341 \\
\hline $4-3,6-8$ & 26.57 & 9.37 E-7 & +35.5 & 332.7 & -15.8 & 164.0 & $\mathrm{R}$ & 23 \\
\hline $4-3,64-66$ & 27.15 & $3.66 \mathrm{E}-7$ & +39.2 & 226.8 & -1.0 & 172.3 & $\mathrm{R}$ & 676 \\
\hline $4-3,89-91$ & 27.40 & 7.21 E-7 & +49.7 & 15.9 & +3.2 & 164.5 & $\mathbf{R}$ & 378 \\
\hline $4-3,114-116$ & 27.65 & 5.37 E-6 & +6.1 & 350.0 & +4.8 & 355.6 & $\mathrm{~N}$ & 130 \\
\hline $4-3,139-141$ & 27.90 & $5.10 \mathrm{E}-6$ & +8.8 & 350.2 & +4.9 & 351.2 & $\mathrm{~N}$ & 189 \\
\hline $4-4,4-6$ & 28.05 & $4.91 \mathrm{E}-6$ & +4.5 & 354.3 & +5.9 & 358.1 & $\mathrm{~N}$ & 235 \\
\hline $4-4,29-31$ & 28.30 & 4.47 E- 6 & +5.3 & 348.6 & +7.2 & 354.9 & $\mathrm{~N}$ & 159 \\
\hline $4-4,54-56$ & 28.55 & $6.05 \mathrm{E}-6$ & +4.7 & 346.3 & +2.9 & 350.2 & $\mathrm{~N}$ & 188 \\
\hline $4-4,79-81$ & 28.80 & $5.49 \mathrm{E}-7$ & +42.6 & 2.8 & +14.2 & 169.4 & $\mathbf{R}$ & 48 \\
\hline $4-4,104-106$ & 29.05 & $1.16 \mathrm{E}-6$ & +51.5 & 178.3 & +7.1 & 173.1 & $\mathbf{R}$ & 393 \\
\hline $4-4,129-131$ & 29.30 & $3.06 \mathrm{E}-7$ & +32.6 & 231.9 & +8.8 & 176.5 & $\mathbf{R}$ & 699 \\
\hline $4-5,4-6$ & 29.55 & $2.54 \mathrm{E}-7$ & +70.0 & 201.3 & +10.4 & 182.8 & $\mathbf{R}$ & 699 \\
\hline $4-5,29-31$ & 29.80 & $5.82 \mathrm{E}-7$ & +62.0 & 125.9 & +4.8 & 173.5 & $\mathbf{R}$ & 499 \\
\hline $4-5,54-56$ & 30.05 & $1.33 \mathrm{E}-6$ & +22.1 & 186.4 & -4.9 & 183.0 & $\mathbf{R}$ & 403 \\
\hline $4-5,79-81$ & 30.30 & $7.84 \mathrm{E}-7$ & +11.7 & 167.6 & -2.4 & 185.9 & $\mathbf{R}$ & 476 \\
\hline $4-5,104-106$ & 30.55 & $4.08 \mathrm{E}-6$ & +4.5 & 357.4 & -2.2 & 3.5 & $\mathrm{~N}$ & 86 \\
\hline $4-5,129-131$ & 30.80 & $5.74 \mathrm{E}-6$ & +7.8 & 0.6 & +7.0 & 8.1 & $\mathrm{~N}$ & 160 \\
\hline $4-6,4-6$ & 31.05 & $1.34 \mathrm{E}-6$ & +25.6 & 332.0 & +17.4 & 191.9 & $\mathbf{R}$ & 47 \\
\hline $4-6,29-31$ & 31.30 & 7.32 E-7 & +25.0 & 308.0 & +13.5 & 193.9 & $\mathrm{R}$ & $>500$ \\
\hline $4-6,5 \%-56$ & 31.55 & $1.40 \mathrm{E}-6$ & +6.7 & 359.4 & +14.8 & 352.4 & $\mathrm{~N}$ & 56 \\
\hline $4-6,79-81$ & 31.80 & $4.78 \mathrm{E}-6$ & +5.1 & 6.3 & +8.5 & 9.8 & $\mathrm{~N}$ & 205 \\
\hline $44-6,104-106$ & 32.05 & $3.09 \mathrm{E}-6$ & +1.6 & 0.8 & +4.2 & 9.1 & $\mathrm{~N}$ & 121 \\
\hline $4-6,139-141$ & 32.40 & $5.28 \mathrm{E}-6$ & +9.4 & 5.5 & +7.1 & 4.7 & $\mathrm{~N}$ & 234 \\
\hline $4-7,4-6$ & 32.55 & $3.51 \mathrm{E}-6$ & -19.3 & 354.1 & +7.3 & 355.4 & $\mathrm{~N}$ & 199 \\
\hline $4-7,27-29$ & 32.78 & 4.94 E-6 & -0.7 & 337.1 & +3.7 & 350.1 & $\mathrm{~N}$ & 123 \\
\hline $5-1,14-16$ & 33.15 & $7.77 \mathrm{E}-7$ & +69.5 & 260.0 & +74.5 & 177.6 & $\mathbf{R}$ & 314 \\
\hline $5-1,36-38$ & 33.37 & 9.93 E-7 & -26.5 & 200.3 & -24.4 & 176.4 & $\mathbf{R}$ & 431 \\
\hline $5-1,60-62$ & 33.61 & $1.07 \mathrm{E}-6$ & -35.7 & 74.5 & -62.5 & 108.7 & $\mathrm{R}$ & 72 \\
\hline $5-1,100-102$ & 34.01 & $7.00 \mathrm{E}-6$ & +60.3 & 186.4 & +61.2 & 186.7 & $\mathbf{R}$ & 47 \\
\hline $5-1,134-136$ & 34.35 & $4.17 \mathrm{E}-7$ & +25.0 & 311.1 & -11.9 & 178.4 & $\mathbf{R}$ & 635 \\
\hline $5-2,4-6$ & 34.55 & $1.43 \mathrm{E}-6$ & +20.1 & 221.8 & +0.4 & 176.6 & R & 464 \\
\hline $5-2,29-31$ & 34.80 & $5.26 \mathrm{E}-7$ & -4.9 & 332.9 & -3.7 & 167.7 & R & 706 \\
\hline $5-2,54-56$ & 35.05 & $4.56 \mathrm{E}-7$ & +20.4 & 229.1 & -1.1 & 161.9 & R & 680 \\
\hline $5-2,79-81$ & 35.30 & $7.00 \mathrm{E}-6$ & -42.6 & 298.5 & +7.1 & 326.9 & $\mathbf{R}$ & 214 \\
\hline $5-2,104-106$ & 35.55 & $7.35 \mathrm{E}-7$ & +14.1 & 199.3 & +2.0 & 166.8 & $\mathbf{R}$ & 772 \\
\hline $5-2,129-131$ & 35.80 & $1.79 \mathrm{E}-6$ & +21.7 & 342.7 & -2.8 & 154.7 & $\mathbf{R}$ & 49 \\
\hline $5-3,4-6$ & 36.05 & $9.19 \mathrm{E}-7$ & -25.7 & 299.9 & -13.3 & 165.7 & $\mathbf{R}$ & 496 \\
\hline $5-3,29-31$ & 36.30 & $1.57 \mathrm{E}-6$ & -36.3 & 209.9 & -12.3 & 159.9 & $\mathbf{R}$ & 526 \\
\hline $5-3,54-56$ & 36.55 & $1.96 \mathrm{E}-6$ & -2.0 & 187.3 & -4.5 & 156.7 & $\mathbf{R}$ & 433 \\
\hline $5-3,79-81$ & 36.80 & $1.51 \mathrm{E}-6$ & +43.4 & 2.0 & -1.6 & 153.6 & $\mathbf{R}$ & 418 \\
\hline $5-3,104-106$ & 37.05 & $1.09 \mathrm{E}-6$ & -36.8 & 171.9 & -9.7 & 162.4 & $\mathbf{R}$ & 677 \\
\hline $5-3,129-131$ & 37.30 & $1.11 \mathrm{E}-6$ & +24.0 & 171.8 & +9.2 & 157.0 & $\mathrm{R}$ & 604 \\
\hline $5-4,4-6$ & 37.55 & $1.30 \mathrm{E}-6$ & -35.2 & 63.9 & -6.9 & 161.7 & $\mathbf{R}$ & 462 \\
\hline $5-4,29-31$ & 37.80 & $6.97 \mathrm{E}-7$ & -20.6 & 329.6 & -1.8 & 165.4 & $\mathbf{R}$ & 762 \\
\hline $5-4,54-56$ & 38.05 & 2.04 E-6 & +52.8 & 316.9 & +4.4 & 169.8 & $\mathbf{R}$ & 485 \\
\hline $5-4,79-81$ & 38.30 & $1.27 \mathrm{E}-6$ & -63.2 & 315.2 & -11.4 & 160.9 & $\mathrm{R}$ & 597 \\
\hline $5-4,104-106$ & 38.55 & $2.77 \mathrm{E}-6$ & +11.4 & 188.0 & -2.9 & 169.5 & $\mathrm{R}$ & 446 \\
\hline $5-4,129-131$ & 38.80 & $1.41 \mathrm{E}-6$ & +66.1 & 310.6 & +4.0 & 170.1 & $\mathbf{R}$ & 506 \\
\hline $5-5,4-6$ & 39.05 & $1.22 \mathrm{E}-6$ & +44.4 & 298.8 & +3.6 & 187.2 & R & 572 \\
\hline $5-5,29-31$ & 39.30 & $1.90 \mathrm{E}-6$ & +16.8 & 43.3 & +13.0 & 183.9 & $\mathrm{R}$ & 347 \\
\hline $5-5,54-56$ & 39.55 & $6.69 \mathrm{E}-7$ & -38.3 & 241.0 & -6.7 & 191.5 & R & 575 \\
\hline $5-5,79-81$ & 39.80 & $1.81 \mathrm{E}-6$ & +27.1 & 340.4 & -1.3 & 192.0 & $\mathrm{R}$ & 347 \\
\hline $5-5,104-106$ & 40.05 & $2.05 \mathrm{E}-6$ & +26.0 & 358.0 & -7.5 & 190.0 & $\mathbf{R}$ & 27 \\
\hline $5-5,129-131$ & 40.30 & $2.76 \mathrm{E}-6$ & +13.3 & 3.0 & -4.9 & 182.7 & $\mathbf{R}$ & 31 \\
\hline $5-6,4-6$ & 40.55 & $5.32 \mathrm{E}-6$ & -1.7 & 19.2 & +4.6 & 28.1 & $\mathrm{~N}$ & 81 \\
\hline $5-6,29-31$ & 40.80 & 7.06 E-6 & +11.8 & 20.2 & +7.9 & 28.8 & $\mathrm{~N}$ & 175 \\
\hline $5-6,54-56$ & 41.05 & $3.71 \mathrm{E}-6$ & -1.1 & 19.0 & +10.4 & 30.4 & $\mathrm{~N}$ & 71 \\
\hline $5-6,79-81$ & 41.30 & $4.45 \mathrm{E}-6$ & -0.2 & 23.4 & +2.8 & 34.2 & $\mathrm{~N}$ & 90 \\
\hline $5-6,104-106$ & 41.55 & $3.80 \mathrm{E}-6$ & +6.8 & 21.4 & -14.1 & 35.0 & $\mathrm{~N}$ & 66 \\
\hline $5-6,129-131$ & 41.80 & $4.31 \mathrm{E}-6$ & +5.0 & 23.9 & -5.1 & 36.6 & $\mathrm{~N}$ & 87 \\
\hline
\end{tabular}


Appendix A. (Continued).

\begin{tabular}{|c|c|c|c|c|c|c|c|c|}
\hline $\begin{array}{l}\text { Core-Section } \\
\text { (interval in } \mathrm{cm} \text { ) }\end{array}$ & $\begin{array}{l}\text { Sub-bottom } \\
\text { depth } \\
\text { (m) }\end{array}$ & $\begin{array}{c}J_{\text {NRM }} \\
(\mathrm{G})\end{array}$ & $I_{\mathrm{NRM}}$ & $D_{\mathrm{NRM}}$ & $I_{\text {stable }}$ & $D_{\text {stable }}$ & Polarity & $\begin{array}{l}\text { MDF } \\
(\mathrm{Oe})\end{array}$ \\
\hline \multicolumn{9}{|l|}{ Hole 574 (Cont.) } \\
\hline $5-7,4-6$ & 42.05 & 3.51 E-6 & +2.2 & 23.1 & +6.3 & 35.2 & $\mathrm{~N}$ & 81 \\
\hline $5-7,29-31$ & 42.30 & 3.27 E-6 & -1.4 & 35.7 & +6.8 & 43.2 & $\mathrm{~N}$ & 153 \\
\hline $6-1,15-17$ & 42.66 & $8.54 \mathrm{E}-7$ & -32.2 & 316.4 & -70.6 & 113.7 & $\mathrm{~N}$ & 484 \\
\hline $6-1,52-54$ & 43.03 & $1.53 \mathrm{E}-6$ & +3.5 & 19.7 & -34.2 & 32.8 & $\mathrm{~N}$ & 179 \\
\hline $6-1,77-79$ & 43.28 & $6.82 \mathrm{E}-7$ & +29.8 & 0.7 & +40.8 & 34.3 & $\mathrm{~N}$ & 124 \\
\hline $6-1,119-121$ & 43.70 & 7.06 E-7 & -30.6 & 332.9 & +39.8 & 182.3 & $\mathbf{R}$ & 28 \\
\hline $6-1,142-144$ & 43.93 & $1.05 \mathrm{E}-6$ & +2.2 & 173.9 & +2.2 & 160.8 & $\mathbf{R}$ & 703 \\
\hline $6-2,4-6$ & 44.05 & 2.45 E-6 & +24.0 & 2.5 & +29.4 & 94.0 & $\mathrm{~N}$ & 40 \\
\hline $6-2,29-31$ & 44.30 & $4.28 \mathrm{E}-6$ & +5.5 & 351.7 & +1.8 & 352.7 & $\mathrm{~N}$ & 227 \\
\hline $6-2,54-56$ & 44.55 & 5.03 E-6 & +11.8 & 337.1 & +12.4 & 344.4 & $\mathrm{~N}$ & 181 \\
\hline $6-2,79-81$ & 44.80 & 5.75 E-6 & +4.5 & 348.1 & -0.5 & 346.9 & $\mathrm{~N}$ & 92 \\
\hline $6-2,104-106$ & 45.05 & 5.92 E-6 & +7.6 & 340.3 & +7.4 & 344.7 & $\mathrm{~N}$ & 208 \\
\hline $6-2,129-131$ & 45.30 & 4.35 E-6 & +10.0 & 347.3 & +11.4 & 0.7 & $\mathrm{~N}$ & 86 \\
\hline $6-3,4-6$ & 45.55 & 2.47 E-6 & -5.8 & 349.2 & +14.2 & 35.4 & $\mathrm{~N}$ & 47 \\
\hline $6-3,29-31$ & 45.80 & 5.83 E-6 & +0.6 & 353.4 & +2.9 & 359.5 & $\mathrm{~N}$ & 285 \\
\hline $6-3,54-56$ & 46.05 & 2.54 E-6 & -1.8 & 345.6 & -4.6 & 2.3 & $\mathrm{~N}$ & 50 \\
\hline $6-3,79-81$ & 46.30 & 4.48 E-6 & +3.6 & 350.5 & +5.9 & 356.1 & $\mathrm{~N}$ & 158 \\
\hline $6-3,104-106$ & 46.55 & $5.24 \mathrm{E}-6$ & -6.4 & 358.3 & -3.3 & 4.1 & $\mathrm{~N}$ & 139 \\
\hline $6-3,129-131$ & 46.80 & 4.65 E-6 & +6.2 & 0.9 & +6.0 & 7.3 & & 138 \\
\hline $6-4,4-6$ & 47.05 & 4.68 E-6 & +5.4 & 359.1 & +5.3 & 358.8 & $\mathrm{~N}$ & 150 \\
\hline $6-4,29-31$ & 47.30 & 1.33 E-6 & +10.8 & 187.1 & +3.4 & 186.1 & $\mathbf{R}$ & 473 \\
\hline $6-4,54-56$ & 47.55 & 8.94 E-8 & -36.0 & 174.1 & +0.8 & 182.3 & $\mathbf{R}$ & $>300$ \\
\hline $6-4,79-81$ & 47.80 & 4.05 E-8 & +27.1 & 348.4 & \multicolumn{2}{|c|}{ no stable } & & \\
\hline $6-4,99-101$ & 48.00 & $4.08 \mathrm{E}-7$ & +17.1 & 16.6 & +29.5 & 15.8 & $\mathrm{~N}$ & 387 \\
\hline $6-4,129-131$ & 48.30 & $1.11 \mathrm{E}-8$ & -17.7 & 236.7 & \multicolumn{2}{|c|}{ no stable } & & \\
\hline $6-5,30-32$ & 48.81 & $1.93 \mathrm{E}-7$ & +19.7 & 132.7 & +9.6 & 202.0 & $\mathbf{R}$ & 23 \\
\hline $6-5,70-72$ & 49.21 & 8.84 E-8 & +44.7 & 202.5 & \multicolumn{2}{|c|}{ no stable } & & \\
\hline $6-5,116-118$ & 49.67 & $1.46 \mathrm{E}-7$ & -41.4 & 254.9 & \multicolumn{2}{|c|}{ no stable } & & \\
\hline $6-6,30-32$ & 50.31 & $1.45 \mathrm{E}-7$ & -27.8 & 261.9 & \multicolumn{2}{|c|}{ no stable } & & \\
\hline $6-6,70-72$ & 50.71 & $1.57 \mathrm{E}-7$ & -31.0 & 241.9 & \multicolumn{2}{|c|}{ no stable } & & \\
\hline $6-6,116-118$ & 51.17 & $2.34 \mathrm{E}-7$ & -59.2 & 258.7 & \multicolumn{2}{|c|}{ no stable } & & \\
\hline $6-7,30-32$ & 51.81 & $1.84 \mathrm{E}-7$ & -55.8 & 258.3 & \multicolumn{2}{|c|}{ no stable } & & 44 \\
\hline $7-1,85-87$ & 52.86 & $2.60 \mathrm{E}-7$ & +0.7 & 2.0 & no $s$ & able & & \\
\hline $7-2,56-58$ & 54.07 & $1.46 \mathrm{E}-7$ & -5.2 & 36.5 & no s & able & & \\
\hline $7-2,65-67$ & 54.16 & $1.75 \mathrm{E}-7$ & -52.4 & 194.7 & no s & able & & \\
\hline $7-2,130-132$ & 54.81 & $1.59 \mathrm{E}-7$ & +2.0 & 61.0 & no s & able & & \\
\hline $7-3,55-57$ & 55.56 & $1.73 \mathrm{E}-7$ & +2.3 & 38.8 & no st & able & & 19 \\
\hline $7-4,15-17$ & 56.66 & $1.59 \mathrm{E}-7$ & +12.9 & 37.5 & no $s$ & able & & 73 \\
\hline $7-4,65-67$ & 57.16 & $1.38 \mathrm{E}-7$ & -37.1 & 174.2 & no $s$ & able & & \\
\hline $7-4,97-99$ & 57.48 & $2.68 \mathrm{E}-7$ & +14.6 & 24.1 & no st & able & & 18 \\
\hline $7-5,34-36$ & 58.35 & $1.30 \mathrm{E}-7$ & +75.7 & 57.5 & no st & able & & \\
\hline $7-5,133-135$ & 59.34 & $7.38 \mathrm{E}-8$ & +1.1 & 36.2 & no $s$ & able & & \\
\hline $7-6,13-15$ & 59.64 & $7.62 \mathrm{E}-7$ & +2.1 & 53.7 & no s & able & & 14 \\
\hline $8-1,92-94$ & 61.93 & $1.59 \mathrm{E}-7$ & +15.6 & 45.9 & no st & able & & \\
\hline $8-2,69-71$ & 63.20 & $2.73 \mathrm{E}-7$ & +2.0 & 48.5 & no $s$ & able & & 17 \\
\hline $8-2,115-117$ & 63.66 & $3.05 \mathrm{E}-7$ & +3.4 & 32.9 & no st & able & & 126 \\
\hline $8-3,33-35$ & 64.34 & 2.44 E-7 & -7.8 & 40.7 & no st & able & & \\
\hline $8-3,113-115$ & 65.14 & $1.33 \mathrm{E}-7$ & -37.1 & 168.3 & no st & able & & \\
\hline $8-4,29-31$ & 65.80 & $4.81 \mathrm{E}-7$ & +29.2 & 202.2 & no $s$ & able & & 205 \\
\hline $8-4,130-132$ & 66.81 & $1.46 \mathrm{E}-7$ & -7.3 & 28.2 & no $s$ & able & & \\
\hline $8-5,117-119$ & 68.18 & $1.58 \mathrm{E}-7$ & +19.6 & 46.5 & no st & able & & \\
\hline $8-6,9-11$ & 68.60 & $1.95 \mathrm{E}-7$ & +11.6 & 34.5 & no $s$ & able & & \\
\hline $8-6,38-40$ & 68.89 & $1.08 \mathrm{E}-7$ & -32.7 & 172.2 & no $s$ & able & & \\
\hline $8-6,125-127$ & 69.76 & 1.65 E-7 & +12.0 & 41.0 & no st & able & & \\
\hline $9-1,65-67$ & 71.16 & $3.64 \mathrm{E}-7$ & -0.3 & 44.8 & no $\mathrm{s}$ & able & & \\
\hline $9-2,5-7$ & 72.06 & $1.90 \mathrm{E}-7$ & +12.8 & 26.8 & no st & able & & 43 \\
\hline $9-2,34-36$ & 72.35 & $1.40 \mathrm{E}-7$ & -43.9 & 72.1 & no st & able & & \\
\hline $9-3,30-32$ & 73.81 & $2.58 \mathrm{E}-7$ & +14.8 & 35.8 & no st & able & & 38 \\
\hline $9-3,144-146$ & 74.95 & $2.20 \mathrm{E}-7$ & +7.1 & 32.4 & no st & able & & \\
\hline $9-4,35-37$ & 75.36 & $3.65 \mathrm{E}-7$ & +0.2 & 76.6 & no st & able & & 85 \\
\hline $9-4,58-60$ & 75.59 & $1.61 \mathrm{E}-7$ & -44.5 & 100.8 & no st & able & & \\
\hline $9-4,85-87$ & 75.86 & 5.88 E-6 & -0.3 & 172.8 & -0.6 & 173.0 & $\mathbf{R}$ & 288 \\
\hline $9-4,118-120$ & 76.19 & 1.41 E-6 & +29.2 & 152.9 & +5.6 & 169.8 & $\mathbf{R}$ & $>1000$ \\
\hline $9-4,135-137$ & 76.36 & $2.55 \mathrm{E}-6$ & +18.9 & 351.6 & +42.4 & 169.2 & $\mathbf{R}$ & 69 \\
\hline $9-5,9-11$ & 76.60 & $1.00 \mathrm{E}-6$ & -8.8 & 52.0 & -1.6 & 172.8 & $\mathrm{R}$ & 361 \\
\hline $9-5,34-36$ & 76.85 & 3.34 E-6 & -3.5 & 16.2 & -7.9 & 3.7 & $\mathrm{~N}$ & 52 \\
\hline $9-5,58-60$ & 77.09 & $4.86 \mathrm{E}-6$ & -4.4 & 10.2 & -8.1 & 3.8 & $\mathrm{~N}$ & 69 \\
\hline $9-5,85-87$ & 77.36 & $5.66 \mathrm{E}-6$ & -4.1 & 18.0 & -2.3 & 9.8 & $\mathrm{~N}$ & 97 \\
\hline $9-5,118-120$ & 77.69 & $2.22 \mathrm{E}-6$ & +0.9 & 31.0 & +17.0 & 173.0 & $R$ & 31 \\
\hline $9-6,9-11$ & 78.10 & 2.40 E-6 & -12.4 & 24.0 & -36.3 & 152.7 & R & 27 \\
\hline
\end{tabular}


Appendix A. (Continued).

\begin{tabular}{|c|c|c|c|c|c|c|c|c|}
\hline $\begin{array}{c}\text { Core-Section } \\
\text { (interval in cm) }\end{array}$ & $\begin{array}{l}\text { Sub-bottom } \\
\text { depth } \\
\text { (m) }\end{array}$ & $\begin{array}{c}J_{\mathrm{NRM}} \\
(\mathrm{G})\end{array}$ & $I_{\mathrm{NRM}}$ & $D_{\text {NRM }}$ & $I_{\text {stable }}$ & $D_{\text {stable }}$ & Polarity & $\begin{array}{l}\text { MDF } \\
\text { (Oe) }\end{array}$ \\
\hline \multicolumn{9}{|l|}{ Hole 574 (Cont.) } \\
\hline $9-6,34-36$ & 78.35 & $3.91 \mathrm{E}-6$ & -10.0 & 6.9 & -11.9 & 357.0 & $\mathrm{~N}$ & 99 \\
\hline $9-6,58-60$ & 78.59 & $4.13 \mathrm{E}-6$ & -7.8 & 9.7 & -6.0 & 1.7 & $\mathrm{~N}$ & 97 \\
\hline $9-6,85-87$ & 78.86 & $6.29 \mathrm{E}-6$ & +4.4 & 16.7 & +9.6 & 6.8 & $\mathrm{~N}$ & 128 \\
\hline $9-6,118-120$ & 79.19 & $5.09 \mathrm{E}-6$ & +4.5 & 4.0 & +4.8 & 354.9 & $\mathrm{~N}$ & 105 \\
\hline $9-7,9-11$ & 79.60 & $2.20 \mathrm{E}-6$ & -9.8 & 33.7 & -6.8 & 19.8 & $\mathrm{~N}$ & 46 \\
\hline $9-7,34-36$ & 79.85 & 3.82 E-6 & -3.9 & 24.2 & -0.3 & 10.1 & $\mathrm{~N}$ & 89 \\
\hline $10-1,23-25$ & 80.24 & $1.81 \mathrm{E}-6$ & +8.1 & 9.6 & +7.7 & 13.2 & $\mathrm{~N}$ & 151 \\
\hline $10-1,50-52$ & 80.51 & $2.38 \mathrm{E}-6$ & +0.8 & 10.1 & -0.1 & 10.4 & $\mathrm{~N}$ & 204 \\
\hline $10-1,77-79$ & 80.78 & $2.50 \mathrm{E}-6$ & +2.7 & 4.6 & +0.5 & 4.8 & $\mathrm{~N}$ & 163 \\
\hline $10-1,102-104$ & 81.03 & $1.01 \mathrm{E}-6$ & -4.1 & 10.0 & -3.6 & 13.2 & $\mathrm{~N}$ & 57 \\
\hline $10-1,125-127$ & 81.26 & $1.97 \mathrm{E}-6$ & +0.4 & 2.0 & -3.2 & 2.2 & $\mathrm{~N}$ & 150 \\
\hline $10-1,147-149$ & 81.48 & $1.47 \mathrm{E}-6$ & +5.3 & 357.2 & +5.4 & 6.0 & $\mathrm{~N}$ & 180 \\
\hline $10-2,23-25$ & 81.74 & $2.01 \mathrm{E}-6$ & -0.1 & 2.0 & +1.2 & 5.6 & $\mathrm{~N}$ & 182 \\
\hline $10-2,50-52$ & 82.01 & $2.58 \mathrm{E}-6$ & -9.6 & 0.0 & -6.0 & 0.3 & $\mathrm{~N}$ & 245 \\
\hline $10-2,77-79$ & 82.28 & $3.36 \mathrm{E}-6$ & -2.6 & 352.1 & -1.4 & 353.9 & $\mathrm{~N}$ & 238 \\
\hline $10-2,102-104$ & 82.53 & $2.51 \mathrm{E}-6$ & -5.7 & 354.0 & -7.1 & 359.9 & $\mathrm{~N}$ & 76 \\
\hline $10-2,125-127$ & 82.76 & $6.32 \mathrm{E}-6$ & -2.2 & 346.4 & -0.8 & 351.2 & $\mathrm{~N}$ & 93 \\
\hline $10-2,147-149$ & 82.98 & $6.98 \mathrm{E}-6$ & +0.3 & 345.9 & +4.9 & 353.2 & $\mathrm{~N}$ & 157 \\
\hline $10-3,23-25$ & 83.24 & $4.90 \mathrm{E}-6$ & +0.8 & 351.5 & +2.6 & 353.6 & $\mathrm{~N}$ & 188 \\
\hline $10-3,50-52$ & 83.51 & $4.45 \mathrm{E}-6$ & -2.0 & 344.4 & +3.6 & 349.2 & $\mathrm{~N}$ & 122 \\
\hline $10-23,77-79$ & 83.78 & $4.84 \mathrm{E}-6$ & +1.0 & 340.2 & +7.6 & 350.7 & $\mathrm{~N}$ & 73 \\
\hline $10-3,102-104$ & 84.03 & $8.96 \mathrm{E}-6$ & +4.0 & 343.1 & +3.5 & 347.6 & $\mathrm{~N}$ & 131 \\
\hline $10-3,125-127$ & 84.26 & $1.32 \mathrm{E}-7$ & +43.1 & 308.2 & \multicolumn{2}{|c|}{ no stable } & & \\
\hline $10-4,13-15$ & 84.64 & $2.91 \mathrm{E}-7$ & +7.8 & 292.8 & \multirow{2}{*}{\multicolumn{2}{|c|}{$\begin{array}{l}\text { no stable } \\
\text { no stable }\end{array}$}} & & \\
\hline $10-4,103-105$ & 85.54 & $6.03 \mathrm{E}-7$ & -7.2 & 305.0 & & & & \\
\hline $10-5,115-117$ & 87.16 & $4.49 \mathrm{E}-7$ & -3.0 & 285.7 & \multicolumn{2}{|c|}{ no stable } & & \\
\hline $10-6,30-32$ & 87.81 & $3.45 \mathrm{E}-7$ & +14.2 & 262.8 & \multicolumn{2}{|c|}{ no stable } & & \\
\hline $10-6,129-131$ & 88.80 & 4.62 E-7 & +2.6 & 283.5 & no $s$ & able & & \\
\hline \multicolumn{9}{|l|}{ Hole 574A } \\
\hline $2-1,14-16$ & 6.45 & $1.53 \mathrm{E}-6$ & +0.7 & 172.2 & -1.7 & 168.4 & $\mathrm{R}$ & 536 \\
\hline $2-1,40-42$ & 6.71 & $1.90 \mathrm{E}-6$ & -7.2 & 166.9 & -8.6 & 163.4 & $\mathbf{R}$ & 478 \\
\hline $2-1,64-66$ & 6.95 & $1.20 \mathrm{E}-6$ & +6.5 & 160.8 & -0.5 & 159.6 & $\mathrm{R}$ & 410 \\
\hline $2-1,89-91$ & 7.20 & $2.02 \mathrm{E}-6$ & +6.2 & 161.7 & +7.5 & 159.0 & $\mathrm{R}$ & 440 \\
\hline $2-1,114-116$ & 7.45 & $4.95 \mathrm{E}-6$ & -2.1 & 152.0 & -2.3 & 151.4 & $\mathrm{R}$ & 361 \\
\hline $2-1,140-142$ & 7.71 & $1.76 \mathrm{E}-6$ & -17.6 & 166.5 & -9.6 & 157.7 & $\mathrm{R}$ & 406 \\
\hline $2-2,15-17$ & 7.96 & $7.68 \mathrm{E}-7$ & -6.8 & 158.9 & -0.6 & 151.1 & $\mathrm{R}$ & 444 \\
\hline $2-2,41-43$ & 8.22 & $2.86 \mathrm{E}-6$ & +1.7 & 173.9 & -4.2 & 170.1 & $\mathbf{R}$ & 380 \\
\hline $2-2,65-67$ & 8.46 & $1.39 \mathrm{E}-6$ & +2.2 & 166.7 & -0.6 & 163.4 & $\mathrm{R}$ & 398 \\
\hline $2-2,90-92$ & 8.71 & $5.20 \mathrm{E}-6$ & +0.7 & 345.1 & +0.1 & 353.4 & $\mathrm{~N}$ & 111 \\
\hline $2-2,115-117$ & 8.96 & $6.70 \mathrm{E}-6$ & +7.7 & 347.6 & +4.2 & 351.4 & $\mathrm{~N}$ & 191 \\
\hline $2-2,141-143$ & 9.22 & $4.39 \mathrm{E}-6$ & +13.2 & 351.6 & +13.1 & 354.8 & $\mathrm{~N}$ & 231 \\
\hline $2-3,15-17$ & 9.46 & $2.05 \mathrm{E}-6$ & -0.4 & 335.0 & -13.8 & 340.4 & $\mathrm{~N}$ & 38 \\
\hline $2-3,41-43$ & 9.72 & $1.23 \mathrm{E}-6$ & +7.7 & 197.9 & +0.1 & 175.8 & $\mathrm{R}$ & 426 \\
\hline $2-3,65-67$ & 9.96 & $1.93 \mathrm{E}-6$ & -7.6 & 220.7 & -8.2 & 199.4 & $\mathbf{R}$ & 401 \\
\hline $2-3,90-92$ & 10.21 & $2.59 \mathrm{E}-6$ & -8.3 & 201.9 & -7.9 & 211.6 & R & 365 \\
\hline $2-3,115-117$ & 10.46 & $1.90 \mathrm{E}-6$ & -16.7 & 213.9 & -6.4 & 228.2 & $\mathrm{R}$ & 370 \\
\hline $2-3,141-143$ & 10.72 & $2.03 \mathrm{E}-6$ & -24.1 & 217.7 & -15.9 & 229.4 & R & 358 \\
\hline $2-4,18-20$ & 10.99 & $1.33 \mathrm{E}-6$ & -24.7 & 64.1 & -30.9 & 71.2 & $\mathrm{~N}$ & 294 \\
\hline $2-4,85-87$ & 11.66 & $1.70 \mathrm{E}-6$ & +13.7 & 44.1 & +8.5 & 45.5 & $\mathrm{~N}$ & 224 \\
\hline $2-5,109-111$ & 13.40 & $1.22 \mathrm{E}-6$ & -25.3 & 351.3 & -27.6 & 346.7 & $\mathrm{~N}$ & 214 \\
\hline $2-5,134-136$ & 13.65 & $3.61 \mathrm{E}-6$ & -4.1 & 2.8 & -2.6 & 10.8 & $\mathrm{~N}$ & 164 \\
\hline $2-6,14-16$ & 13.95 & $3.88 \mathrm{E}-6$ & +8.0 & 14.0 & +7.9 & 14.5 & $\mathrm{~N}$ & 202 \\
\hline $2-6,40-42$ & 14.21 & $5.30 \mathrm{E}-6$ & +0.2 & 7.2 & +4.9 & 13.0 & $\mathrm{~N}$ & 178 \\
\hline $2-6,64-66$ & 14.45 & $6.05 \mathrm{E}-6$ & +6.9 & 16.2 & +2.5 & 17.6 & $\mathrm{~N}$ & 238 \\
\hline $2-6,89-91$ & 14.70 & $5.26 \mathrm{E}-6$ & +12.6 & 13.4 & +7.5 & 12.1 & $\mathrm{~N}$ & 225 \\
\hline $2-6,109-111$ & 14.90 & $4.20 \mathrm{E}-6$ & +2.5 & 10.5 & +4.9 & 10.2 & $\mathrm{~N}$ & 226 \\
\hline $2-6,134-136$ & 15.15 & 7.31 E-7 & +13.7 & 190.4 & +6.8 & 188.3 & $\mathrm{R}$ & 488 \\
\hline $3-1,24-26$ & 15.75 & $8.50 \mathrm{E}-7$ & -31.2 & 223.0 & -16.9 & 225.6 & $\mathrm{R}$ & 430 \\
\hline $3-1,49-51$ & 16.00 & $3.32 \mathrm{E}-7$ & +38.8 & 246.0 & -2.4 & 221.0 & R & 638 \\
\hline $3-1,74-76$ & 16.25 & $5.26 \mathrm{E}-7$ & +14.3 & 223.4 & -2.9 & 219.9 & R & 523 \\
\hline $3-1,100-102$ & 16.51 & $1.28 \mathrm{E}-6$ & -6.4 & 227.6 & -9.1 & 220.9 & R & 388 \\
\hline $3-1,124-126$ & 16.75 & $1.10 \mathrm{E}-6$ & +17.7 & 222.8 & +6.8 & 217.1 & $\mathrm{R}$ & 397 \\
\hline $3-2,2-4$ & 17.03 & $1.41 \mathrm{E}-6$ & -1.6 & 204.1 & -5.8 & 201.8 & $\mathrm{R}$ & 437 \\
\hline $3-2,24-26$ & 17.25 & $2.01 \mathrm{E}-6$ & -2.8 & 211.0 & -8.3 & 207.6 & $\mathrm{R}$ & 373 \\
\hline $3-2,49-51$ & 17.50 & $2.35 \mathrm{E}-6$ & +0.7 & 207.4 & +0.3 & 205.1 & $\hat{R}$ & 423 \\
\hline $3-2,80-82$ & 17.81 & $1.88 \mathrm{E}-6$ & -20.0 & 200.4 & -17.3 & 197.5 & $\mathbf{R}$ & 419 \\
\hline $3-2,104-106$ & 18.05 & $4.04 \mathrm{E}-7$ & +6.5 & 225.5 & -5.5 & 186.7 & R & 496 \\
\hline $3-2,134-136$ & 18.35 & $2.24 \mathrm{E}-6$ & -0.6 & 194.4 & -3.9 & 186.0 & R & 375 \\
\hline $3-3,14-16$ & 18.65 & $1.53 \mathrm{E}-6$ & +17.4 & 185.2 & +7.1 & 177.9 & R & 408 \\
\hline $3-3,40-42$ & 18.91 & $2.02 \mathrm{E}-6$ & -12.2 & 182.6 & -12.3 & 175.9 & $\mathrm{R}$ & 395 \\
\hline
\end{tabular}


Appendix A. (Continued).

\begin{tabular}{|c|c|c|c|c|c|c|c|c|}
\hline $\begin{array}{l}\text { Core-Section } \\
\text { (interval in } \mathrm{cm} \text { ) }\end{array}$ & $\begin{array}{l}\text { Sub-bottom } \\
\text { depth } \\
\text { (m) }\end{array}$ & $\underset{(\mathrm{NR})}{J_{\mathrm{NRM}}}$ & $I_{\mathrm{NRM}}$ & $D_{\text {NRM }}$ & $I_{\text {stable }}$ & $D_{\text {stable }}$ & Polarity & $\begin{array}{l}\text { MDF } \\
(\mathrm{Oe})\end{array}$ \\
\hline \multicolumn{9}{|l|}{ Hole 574A (Cont.) } \\
\hline $3-3,64-66$ & 19.15 & $2.43 \mathrm{E}-6$ & -1.1 & 177.0 & -7.7 & 169.7 & $\mathbf{R}$ & 403 \\
\hline $3-3,89-91$ & 19.40 & $2.20 \mathrm{E}-6$ & -0.2 & 175.6 & -3.6 & 164.0 & $\mathrm{R}$ & 420 \\
\hline $3-3,114-116$ & 19.65 & $2.22 \mathrm{E}-6$ & +12.8 & 184.6 & -4.4 & 166.6 & $\mathrm{R}$ & 440 \\
\hline $3-3,139-141$ & 19.90 & $1.59 \mathrm{E}-6$ & +41.0 & 151.2 & +12.8 & 160.6 & R & 476 \\
\hline $3-4,14-16$ & 20.15 & 1.95 E-6 & -11.9 & 156.3 & -8.2 & 150.4 & $\mathbf{R}$ & 439 \\
\hline $3-4,40-42$ & 20.41 & 7.77 E-7 & +15.0 & 235.6 & -0.9 & 152.3 & $\mathbf{R}$ & 459 \\
\hline $3-4,64-66$ & 20.65 & $1.35 \mathrm{E}-6$ & -2.9 & 141.5 & -3.6 & 145.2 & $\mathbf{R}$ & 441 \\
\hline $3-4,89-91$ & 20.90 & 1.11 E-6 & -14.1 & 298.6 & -8.3 & 143.2 & $\mathbf{R}$ & 338 \\
\hline $3-4,114-116$ & 21.15 & $1.18 \mathrm{E}-6$ & +0.0 & 162.2 & -0.8 & 143.5 & $\mathbf{R}$ & 476 \\
\hline $3-4,140-142$ & 21.41 & $3.31 \mathrm{E}-6$ & +16.7 & 334.2 & +13.9 & 336.2 & $\mathrm{~N}$ & 82 \\
\hline $3-5,14-16$ & 21.65 & $4.08 \mathrm{E}-6$ & +14.1 & 325.3 & +12.5 & 321.0 & $\mathrm{~N}$ & 117 \\
\hline $3-5,40-42$ & 21.91 & $1.57 \mathrm{E}-7$ & +30.7 & 332.8 & +38.0 & 329.6 & $\mathrm{~N}$ & 514 \\
\hline $3-5,108-110$ & 22.59 & $5.75 \mathrm{E}-7$ & +74.0 & 84.1 & +61.0 & 81.1 & $\mathrm{~N}$ & 377 \\
\hline $3-5,136-138$ & 22.87 & $6.40 \mathrm{E}-7$ & +38.4 & 113.0 & \multirow{2}{*}{\multicolumn{2}{|c|}{$\begin{array}{l}\text { no stable } \\
\text { no stable }\end{array}$}} & & 311 \\
\hline $3-6,14-16$ & 23.15 & $5.36 \mathrm{E}-7$ & -53.0 & 249.7 & & & & \\
\hline $3-6,80-82$ & 23.81 & $3.00 \mathrm{E}-7$ & -11.1 & 226.8 & \multicolumn{2}{|c|}{ no stable } & & \\
\hline $4-1,10-12$ & 24.01 & $1.74 \mathrm{E}-6$ & -63.2 & 154.7 & \multicolumn{2}{|c|}{ no stable } & & 334 \\
\hline $4-1,49-51$ & 24.40 & $1.14 \mathrm{E}-6$ & -56.8 & 167.8 & -26.5 & 290.9 & $\mathrm{~N}$ & 46 \\
\hline $4-1,80-82$ & 24.71 & $4.68 \mathrm{E}-6$ & -8.0 & 359.4 & -14.8 & 4.1 & $\mathrm{~N}$ & 144 \\
\hline $4-1,103-105$ & 24.94 & $8.13 \mathrm{E}-7$ & +46.5 & 220.9 & -1.2 & 182.1 & R & 463 \\
\hline $4-1,129-131$ & 25.20 & $4.33 \mathrm{E}-7$ & +23.1 & 238.7 & -8.7 & 166.4 & $\mathbf{R}$ & $>500$ \\
\hline $4-2,4-6$ & 25.45 & $7.53 \mathrm{E}-7$ & +41.3 & 216.6 & -6.0 & 164.3 & $\mathrm{R}$ & 529 \\
\hline $4-2,29-31$ & 25.70 & $1.39 \mathrm{E}-6$ & +44.9 & 197.1 & +6.6 & 166.0 & $\mathbf{R}$ & 496 \\
\hline $4-2,54-56$ & 25.95 & 8.99 E-7 & +47.1 & 335.7 & -3.2 & 172.6 & $\mathbf{R}$ & 451 \\
\hline $4-2,80-82$ & 26.21 & $4.22 \mathrm{E}-7$ & -11.3 & 303.8 & -14.4 & 174.0 & $\mathbf{R}$ & 539 \\
\hline $4-2,104-106$ & 26.45 & 7.76 E-7 & -6.4 & 162.0 & -15.2 & 179.0 & R & 532 \\
\hline $4-2,129-131$ & 26.70 & $3.40 \mathrm{E}-7$ & -23.1 & 236.2 & -6.5 & 173.3 & $\mathbf{R}$ & 584 \\
\hline $4-3,4-6$ & 26.95 & $4.29 \mathrm{E}-6$ & +10.8 & 5.5 & +1.7 & 5.8 & $\mathrm{~N}$ & 108 \\
\hline $4-3,29-31$ & 27.20 & $4.63 \mathrm{E}-6$ & +12.1 & 7.3 & +5.0 & 8.5 & $\mathrm{~N}$ & 142 \\
\hline $4-3,54-56$ & 27.45 & $4.94 \mathrm{E}-6$ & +10.5 & 8.2 & +9.7 & 6.1 & $\mathrm{~N}$ & 211 \\
\hline $4-3,80-82$ & 27.71 & $6.16 \mathrm{E}-6$ & +15.5 & 5.0 & +14.3 & 5.6 & $\mathrm{~N}$ & 208 \\
\hline $4-3,104-106$ & 27.95 & $8.60 \mathrm{E}-7$ & -18.2 & 194.3 & -26.3 & 184.4 & $R$ & 486 \\
\hline $4-3,129-131$ & 28.20 & 8.74 E-7 & +5.1 & 187.2 & -9.5 & 182.2 & $\mathbf{R}$ & 439 \\
\hline $4-4,4-6$ & 28.45 & 9.54 E-7 & +10.2 & 2.2 & -17.4 & 177.6 & $\mathbf{R}$ & 42 \\
\hline $4-4,29-31$ & 28.70 & $2.85 \mathrm{E}-7$ & +33.7 & 197.8 & -11.3 & 182.7 & $\mathbf{R}$ & 600 \\
\hline $4-4,54-56$ & 28.95 & $1.38 \mathrm{E}-6$ & -10.1 & 3.9 & -26.7 & 190.8 & $\mathbf{R}$ & 30 \\
\hline $4-4,80-82$ & 29.21 & $5.31 \mathrm{E}-6$ & +10.5 & 356.1 & +3.8 & 0.9 & $\mathrm{~N}$ & 117 \\
\hline $4-4,104-106$ & 29.45 & $3.13 \mathrm{E}-6$ & +13.3 & 357.6 & +7.2 & 357.9 & $\mathrm{~N}$ & 68 \\
\hline $4-4,129-131$ & 29.70 & $1.55 \mathrm{E}-6$ & +22.5 & 350.4 & +52.1 & 175.3 & $\mathrm{R}$ & 48 \\
\hline $4-5,4-6$ & 29.95 & $5.96 \mathrm{E}-7$ & +26.6 & 11.1 & -13.0 & 179.9 & $\mathbf{R}$ & 461 \\
\hline $4-5,37-39$ & 30.28 & $2.90 \mathrm{E}-6$ & +6.4 & 0.4 & -7.7 & 4.8 & $\mathrm{~N}$ & 73 \\
\hline $4-5,68-70$ & 30.59 & $3.44 \mathrm{E}-6$ & +4.1 & 356.8 & +6.2 & 355.4 & $\mathrm{~N}$ & 120 \\
\hline $4-5,113-115$ & 31.04 & $3.43 \mathrm{E}-6$ & +12.6 & 2.4 & +9.8 & 1.8 & $\mathrm{~N}$ & 189 \\
\hline $4-5,135-137$ & 31.26 & $3.07 \mathrm{E}-6$ & +6.6 & 3.7 & +2.7 & 2.3 & $\mathrm{~N}$ & 90 \\
\hline $4-6,5-7$ & 31.46 & $3.10 \mathrm{E}-6$ & +4.0 & 4.3 & +6.1 & 6.8 & $\mathrm{~N}$ & 139 \\
\hline $4-6,27-29$ & 31.68 & $7.23 \mathrm{E}-7$ & +17.4 & 162.6 & -4.6 & 180.9 & R & 417 \\
\hline $4-6,51-53$ & 31.92 & $3.25 \mathrm{E}-7$ & +10.6 & 181.8 & -8.2 & 185.2 & $\mathbf{R}$ & 472 \\
\hline $4-6,75-77$ & 32.16 & $1.02 \mathrm{E}-6$ & +26.3 & 128.7 & +1.1 & 184.9 & $\mathbf{R}$ & 312 \\
\hline $4-6,104-106$ & 32.45 & $2.67 \mathrm{E}-6$ & +48.4 & 111.9 & \multicolumn{2}{|c|}{ no stable } & & 266 \\
\hline $5-1,115-117$ & 34.26 & $3.34 \mathrm{E}-7$ & +42.4 & 290.6 & -9.5 & 188.3 & $\mathbf{R}$ & $>600$ \\
\hline $5-1,142-144$ & 34.53 & $1.20 \mathrm{E}-6$ & -32.7 & 176.3 & -15.3 & 183.9 & R & 513 \\
\hline $5-2,14-16$ & 34.75 & $5.04 \mathrm{E}-7$ & +55.2 & 356.0 & -10.4 & 176.5 & $\mathrm{R}$ & 550 \\
\hline $5-2,40-42$ & 35.01 & $1.14 \mathrm{E}-6$ & +33.4 & 172.2 & -1.5 & 187.7 & $\mathbf{R}$ & 480 \\
\hline $5-2,64-66$ & 35.25 & $9.91 \mathrm{E}-7$ & +19.4 & 281.4 & -0.6 & 178.0 & $\mathrm{R}$ & 522 \\
\hline $5-2,89-91$ & 35.50 & $7.74 \mathrm{E}-7$ & -9.0 & 127.9 & -15.5 & 180.7 & $\mathrm{R}$ & 553 \\
\hline $5-2,114-116$ & 35.75 & $1.96 \mathrm{E}-6$ & +14.5 & 197.2 & -5.6 & 185.7 & $\mathrm{R}$ & 488 \\
\hline $5-2,140-142$ & 36.01 & 5.97 E-7 & +20.2 & 194.3 & -11.7 & 176.7 & $\mathbf{R}$ & 496 \\
\hline $5-3,14-16$ & 36.25 & $1.03 \mathrm{E}-6$ & +21.4 & 143.6 & -0.6 & 181.6 & R & 577 \\
\hline $5-3,40-42$ & 36.51 & $9.56 \mathrm{E}-7$ & -4.6 & 178.2 & -1.6 & 178.8 & $\mathrm{R}$ & 625 \\
\hline $5-3,64-66$ & 36.75 & $2.50 \mathrm{E}-6$ & -10.2 & 176.4 & -7.8 & 180.8 & $\mathbf{R}$ & 517 \\
\hline $5-3,89-91$ & 37.00 & $2.20 \mathrm{E}-6$ & +4.6 & 173.6 & -1.3 & 183.4 & $\mathrm{R}$ & 488 \\
\hline $5-3,114-116$ & 37.25 & $1.40 \mathrm{E}-6$ & -15.7 & 179.1 & -1.8 & 174.2 & $\mathbf{R}$ & 497 \\
\hline $5-3,140-142$ & 37.51 & 1.33 E-6 & -3.9 & 170.0 & -2.4 & 178.1 & $\mathbf{R}$ & 571 \\
\hline $5-4,14-16$ & 37.75 & $1.06 \mathrm{E}-6$ & +21.3 & 177.7 & +3.5 & 181.4 & $\mathbf{R}$ & 615 \\
\hline $5-4,40-42$ & 38.01 & $6.63 \mathrm{E}-7$ & +41.6 & 217.7 & -7.5 & 188.5 & $\mathbf{R}$ & 630 \\
\hline $5-4,64-66$ & 38.25 & $1.21 \mathrm{E}-6$ & +70.5 & 49.6 & +7.1 & 186.9 & $\mathrm{R}$ & 471 \\
\hline $5-4,89-91$ & 38.50 & $1.40 \mathrm{E}-6$ & -28.0 & 174.3 & -10.6 & 191.5 & $\mathbf{R}$ & 522 \\
\hline $5-4,114-116$ & 38.75 & 3.84 E-7 & +29.6 & 297.8 & +3.9 & 210.1 & $\mathrm{R}$ & $>800$ \\
\hline $5-4,140-142$ & 39.01 & $4.96 \mathrm{E}-7$ & +6.8 & 189.0 & -6.7 & 185.7 & $\mathbf{R}$ & $>800$ \\
\hline $5-5,14-16$ & 39.25 & $1.68 \mathrm{E}-6$ & +31.1 & 345.1 & +19.7 & 199.0 & R & 33 \\
\hline $5-5,40-42$ & 39.51 & $2.31 \mathrm{E}-6$ & +2.5 & 347.8 & +6.0 & 345.7 & $\mathrm{~N}$ & 85 \\
\hline $5-5,64-66$ & 39.75 & $5.20 \mathrm{E}-6$ & +10.3 & 1.0 & +15.6 & 353.7 & $\mathrm{~N}$ & 180 \\
\hline
\end{tabular}


Appendix A. (Continued).

\begin{tabular}{|c|c|c|c|c|c|c|c|c|}
\hline $\begin{array}{l}\text { Core-Section } \\
\text { (interval in } \mathrm{cm} \text { ) }\end{array}$ & $\begin{array}{l}\text { Sub-bottom } \\
\text { depth } \\
\text { (m) }\end{array}$ & $\underset{(\mathrm{G})}{J_{\mathrm{NRM}}}$ & $I_{\mathrm{NRM}}$ & $D_{\mathrm{NRM}}$ & $I_{\text {stable }}$ & $D_{\text {stable }}$ & Polarity & $\begin{array}{l}\text { MDF } \\
\text { (Oe) }\end{array}$ \\
\hline \multicolumn{9}{|l|}{ Hole 574A (Cont.) } \\
\hline $5-5,89-91$ & 40.00 & 3.65 E-6 & +24.0 & 357.9 & +22.8 & 342.6 & $\mathrm{~N}$ & 73 \\
\hline $5-5,114-116$ & 40.25 & $4.69 \mathrm{E}-6$ & +15.4 & 347.3 & +9.7 & 349.8 & $\mathrm{~N}$ & 165 \\
\hline $5-5,140-142$ & 40.51 & 2.54 E-6 & +3.0 & 354.8 & +7.1 & 346.1 & $\mathrm{~N}$ & 82 \\
\hline $5-6,14-16$ & 40.75 & 2.87 E-6 & +1.3 & 5.5 & -0.8 & 350.6 & $\mathrm{~N}$ & 89 \\
\hline $5-6,40-42$ & 41.01 & 3.07 E-6 & +13.0 & 3.1 & +14.8 & 355.6 & $\mathrm{~N}$ & 97 \\
\hline $5-6,64-66$ & 41.25 & 3.95 E-6 & +9.9 & 349.4 & +2.5 & 352.2 & $\mathrm{~N}$ & 181 \\
\hline $5-6,89-91$ & 41.50 & $1.88 \mathrm{E}-6$ & +8.7 & 6.1 & -4.6 & 346.5 & $\mathrm{~N}$ & 70 \\
\hline $5-6,122-124$ & 41.83 & 8.82 E-7 & +33.5 & 32.6 & +1.9 & 185.9 & $\mathbf{R}$ & 42 \\
\hline $5-6,144-146$ & 42.05 & $9.30 \mathrm{E}-7$ & +16.6 & 148.2 & -9.0 & 184.0 & $\mathbf{R}$ & 635 \\
\hline $5-7,9-11$ & 42.20 & $1.29 \mathrm{E}-6$ & -63.3 & 333.0 & -11.5 & 86.2 & $\mathbf{R}$ & 328 \\
\hline $6-1,13-15$ & 42.54 & 3.94 E-6 & -0.4 & 358.2 & -5.3 & 358.5 & $\mathrm{~N}$ & 133 \\
\hline $6-1,41-43$ & 42.82 & 3.75 E-6 & -0.3 & 354.0 & -14.2 & 353.9 & $\mathrm{~N}$ & 129 \\
\hline $6-1,65-67$ & 43.06 & 2.84 E-6 & +3.3 & 319.8 & +1.9 & 4.3 & $\mathrm{~N}$ & 85 \\
\hline $6-1,90-92$ & 43.31 & $3.25 \mathrm{E}-6$ & +6.9 & 1.3 & +6.7 & 1.4 & $\mathrm{~N}$ & 197 \\
\hline $6-1,115-117$ & 43.56 & 3.74 E-6 & +16.1 & 355.7 & +15.1 & 356.3 & $\mathbf{N}$ & 150 \\
\hline $6-1,141-143$ & 43.82 & $1.48 \mathrm{E}-7$ & -17.1 & 181.9 & -20.5 & 185.8 & $\mathbf{R}$ & 362 \\
\hline $6-2,11-13$ & 44.02 & $1.14 \mathrm{E}-7$ & -27.7 & 189.2 & -28.0 & 190.6 & $\mathbf{R}$ & $>400$ \\
\hline $6-2,41-43$ & 44.32 & $2.07 \mathrm{E}-7$ & -38.9 & 181.8 & -1.9 & 180.9 & $\mathbf{R}$ & 353 \\
\hline $6-2,65-67$ & 44.56 & $1.11 \mathrm{E}-7$ & -11.0 & 177.4 & -35.4 & 180.7 & $\mathbf{R}$ & 463 \\
\hline $6-2,90-92$ & 44.81 & $2.41 \mathrm{E}-7$ & +6.6 & 334.8 & -1.8 & 329.0 & $\mathrm{~N}$ & 381 \\
\hline $6-2,115-117$ & 45.06 & $6.41 \mathrm{E}-8$ & -24.7 & 195.9 & -11.3 & 196.7 & $\mathbf{R}$ & 87 \\
\hline $6-2,140-142$ & 45.31 & 4.40 E- 8 & -52.8 & 138.5 & -4.2 & 170.0 & $\mathbf{R}$ & 20 \\
\hline $6-3,13-15$ & 45.54 & $1.20 \mathrm{E}-7$ & +51.0 & 248.4 & \multicolumn{2}{|c|}{ no stable } & & 68 \\
\hline $6-3,41-43$ & 45.82 & $6.06 \mathrm{E}-8$ & -4.1 & 165.2 & \multicolumn{2}{|c|}{ no stable } & & \\
\hline $6-3,68-70$ & 46.09 & 3.54 E-8 & -66.5 & 8.0 & \multicolumn{2}{|c|}{ no stable } & & \\
\hline $6-3,85-87$ & 46.26 & $6.98 \mathrm{E}-8$ & +80.6 & 194.9 & \multicolumn{2}{|c|}{ no stable } & & 39 \\
\hline $6-3,130-132$ & 46.71 & $4.46 \mathrm{E}-8$ & -65.6 & 39.9 & \multicolumn{2}{|c|}{ no stable } & & \\
\hline $6-4,13-15$ & 47.04 & $5.50 \mathrm{E}-7$ & +13.4 & 80.3 & \multicolumn{2}{|c|}{ no stable } & & \\
\hline $6-4,55-57$ & 47.46 & $2.20 \mathrm{E}-7$ & +13.1 & 95.6 & \multicolumn{2}{|c|}{ no stable } & & \\
\hline $6-4,95-97$ & 47.86 & $4.63 \mathrm{E}-8$ & +36.2 & 216.3 & no st & able & & \\
\hline $6-4,137-139$ & 48.28 & $2.07 \mathrm{E}-7$ & +31.6 & 106.3 & no st & able & & \\
\hline $6-5,13-15$ & 48.54 & 5.70 E-8 & +18.6 & 88.9 & no st & able & & \\
\hline $6-5,57-59$ & 48.98 & $1.49 \mathrm{E}-7$ & -68.7 & 56.4 & no st & able & & \\
\hline $6-5,105-107$ & 49.46 & $1.06 \mathrm{E}-7$ & -53.2 & 121.7 & no st & able & & \\
\hline $6-5,137-139$ & 49.78 & $1.63 \mathrm{E}-7$ & -80.5 & 143.3 & no st & able & & \\
\hline $6-6,13-15$ & 50.04 & 8.01 E-8 & -10.0 & 156.7 & no st & able & & \\
\hline $6-6,84-86$ & 50.75 & $6.56 \mathrm{E}-8$ & +35.1 & 88.8 & no st & able & & \\
\hline $6-6,118-120$ & 51.09 & $1.28 \mathrm{E}-7$ & -62.0 & 125.2 & no st & able & & \\
\hline $7-1,85-87$ & 52.76 & $1.44 \mathrm{E}-7$ & -48.3 & 149.9 & no st & able & & \\
\hline $7-1,126-128$ & 53.17 & $2.06 \mathrm{E}-7$ & +51.1 & 187.4 & no st & able & & \\
\hline $7-2,8-10$ & 53.49 & 9.96 E-8 & -46.1 & 23.6 & no st & able & & \\
\hline $7-2,45-47$ & 53.86 & $3.14 \mathrm{E}-7$ & -63.0 & 45.6 & no st & able & & \\
\hline $7-2,85-87$ & 54.26 & $2.95 \mathrm{E}-7$ & -83.4 & 354.9 & no st & able & & \\
\hline $7-2,125-127$ & 54.66 & $3.64 \mathrm{E}-7$ & -52.9 & 42.4 & no st & able & & \\
\hline $7-3,8-10$ & 54.99 & $1.61 \mathrm{E}-7$ & -59.9 & 302.7 & no st & able & & \\
\hline $7-3,35-37$ & 55.26 & $1.03 \mathrm{E}-7$ & +63.4 & 259.8 & no st & able & & \\
\hline $7-3,69-71$ & 55.60 & $6.60 \mathrm{E}-8$ & +39.5 & 348.7 & no st & able & & \\
\hline $7-3,108-110$ & 55.99 & 5.80 E-8 & -31.0 & 111.3 & no st & able & & \\
\hline $7-4,15-17$ & 56.56 & 1.87 E-7 & -35.9 & 253.0 & no st & able & & \\
\hline $7-4,51-53$ & 56.92 & $5.78 \mathrm{E}-8$ & -46.4 & 38.4 & no st & able & & \\
\hline $7-4,86-88$ & 57.27 & $6.61 \mathrm{E}-8$ & +41.0 & 83.5 & no st & able & & \\
\hline $7-4,121-123$ & 57.62 & 4.95 E- 8 & -63.7 & 142.7 & no st & able & & \\
\hline $7-5,15-17$ & 58.06 & 6.47 E- 8 & +26.5 & 173.4 & no st & able & & \\
\hline $7-5,51-53$ & 58.42 & 1.19 E-7 & +51.7 & 153.3 & no st & able & & \\
\hline $7-5,96-98$ & 58.87 & $1.50 \mathrm{E}-7$ & -55.9 & 180.1 & no st & able & & \\
\hline $7-5,134-136$ & 59.25 & $2.05 \mathrm{E}-8$ & +31.3 & 234.5 & no st. & able & & \\
\hline $7-6,17-19$ & 59.58 & 5.44 E-8 & +46.7 & 185.5 & no st & able & & \\
\hline $7-6,53-55$ & 59.94 & $1.45 \mathrm{E}-7$ & +80.6 & 234.9 & no st. & able & & \\
\hline $7-6,65-67$ & 60.06 & 4.36 E-8 & -19.5 & 99.6 & no st. & able & & \\
\hline $7-6,97-99$ & 60.38 & 7.05 E-8 & -78.1 & 105.5 & no st. & able & & \\
\hline $7-6,134-136$ & 60.75 & $5.98 \mathrm{E}-8$ & -69.9 & 15.0 & no st: & able & & \\
\hline $8-1,95-97$ & 62.36 & $1.50 \mathrm{E}-7$ & -80.6 & 263.0 & no st. & able & & \\
\hline $8-1,134-136$ & 62.75 & $5.86 \mathrm{E}-8$ & +27.5 & 90.4 & no st & able & & \\
\hline $8-2,32-34$ & 63.23 & 4.74 E-8 & -20.2 & 324.9 & no st: & able & & \\
\hline $8-2,108-110$ & 63.99 & $1.96 \mathrm{E}-8$ & -15.5 & 22.7 & no st: & able & & \\
\hline $8-3,64-66$ & 65.05 & $1.10 \mathrm{E}-7$ & -16.2 & 264.6 & no st: & able & & \\
\hline $8-3,103-105$ & 65.44 & 4.54 E-8 & -30.7 & 55.0 & no st: & able & & \\
\hline $8-3,144-146$ & 65.85 & $1.40 \mathrm{E}-7$ & +52.7 & 81.3 & no st: & able & & \\
\hline $8-4,44-46$ & 66.35 & $1.29 \mathrm{E}-7$ & -51.2 & 239.5 & no st: & able & & \\
\hline $8-4,68-70$ & 66.59 & 2.99 E-8 & -1.0 & 43.9 & no st: & able & & \\
\hline $8-4,103-105$ & 66.94 & $1.21 \mathrm{E}-7$ & -38.3 & 165.7 & no st: & able & & \\
\hline
\end{tabular}


Appendix A. (Continued).

\begin{tabular}{|c|c|c|c|c|c|c|c|c|}
\hline $\begin{array}{l}\text { Core-Section } \\
\text { (interval in cm) }\end{array}$ & $\begin{array}{l}\text { Sub-bottom } \\
\text { depth } \\
\text { (m) }\end{array}$ & $\underset{(\mathrm{G})}{J_{\mathrm{NRM}}}$ & $I_{\mathrm{NRM}}$ & $D_{\text {NRM }}$ & $I_{\text {stable }}$ & $D_{\text {stable }}$ & Polarity & $\begin{array}{l}\text { MDF } \\
(\mathrm{Oe})\end{array}$ \\
\hline \multicolumn{9}{|l|}{ Hole 574A (Cont.) } \\
\hline $8-4,145-147$ & 67.36 & $3.71 \mathrm{E}-8$ & +54.5 & 356.3 & \multicolumn{2}{|c|}{ no stable } & & \\
\hline $8-5,23-25$ & 67.64 & $6.01 \mathrm{E}-8$ & -24.1 & 336.6 & \multicolumn{2}{|c|}{ no stable } & & \\
\hline $8-5,52-54$ & 67.93 & $1.35 \mathrm{E}-7$ & -70.1 & 94.3 & \multirow{2}{*}{\multicolumn{2}{|c|}{$\begin{array}{l}\text { no stable } \\
\text { no stable }\end{array}$}} & & \\
\hline $8-5,91-93$ & 68.32 & $1.77 \mathrm{E}-8$ & -53.5 & 28.3 & & & & \\
\hline $8-5,123-125$ & 68.64 & $1.12 \mathrm{E}-7$ & -75.9 & 98.0 & \multicolumn{2}{|c|}{$\begin{array}{l}\text { no stable } \\
\text { no stable }\end{array}$} & & \\
\hline $8-6,15-17$ & 69.06 & $9.21 \mathrm{E}-8$ & -35.5 & 260.9 & \multicolumn{2}{|c|}{ no stable } & & \\
\hline $8-6,46-48$ & 69.37 & $2.18 \mathrm{E}-7$ & +23.0 & 180.1 & \multicolumn{2}{|c|}{ no stable } & & \\
\hline $8-6,78-80$ & 69.69 & $1.46 \mathrm{E}-7$ & +77.8 & 174.5 & \multicolumn{2}{|c|}{ no stable } & & \\
\hline $8-6,112-114$ & 70.03 & $1.04 \mathrm{E}-7$ & +37.3 & 356.4 & no s & table & & \\
\hline $9-1,25-27$ & 70.66 & 2.27 E-7 & +19.6 & 204.9 & no $s$ & table & & \\
\hline $9-1,128-130$ & 71.69 & $1.21 \mathrm{E}-7$ & -50.6 & 174.7 & no st & able & & \\
\hline $9-2,105-107$ & 72.96 & $1.93 \mathrm{E}-7$ & +14.4 & 227.0 & no $s$ & able & & \\
\hline $9-3,67-69$ & 74.08 & $1.87 \mathrm{E}-7$ & +3.4 & 222.9 & no $s$ & able & & \\
\hline $9-3,135-137$ & 74.76 & 9.09 E-8 & +32.1 & 266.1 & no $s$ & able & & \\
\hline $9-3,143-145$ & 74.84 & $2.43 \mathrm{E}-7$ & -9.4 & 211.0 & no $s$ & able & & \\
\hline $9-4,26-28$ & 75.17 & 6.87 E-8 & -16.2 & 72.8 & no $s$ & able & & \\
\hline $9-4,70-72$ & 75.61 & $2.38 \mathrm{E}-8$ & -56.3 & 38.8 & no $s$ & able & & \\
\hline $9-4,115-117$ & 76.06 & 6.65 E-6 & -21.6 & 168.0 & -21.0 & 169.0 & $\mathbf{R}$ & 287 \\
\hline $9-4,144-146$ & 76.35 & $6.34 \mathrm{E}-7$ & +48.0 & 355.3 & -15.1 & 173.7 & $\mathbf{R}$ & $>600$ \\
\hline $9-5,15-17$ & 76.56 & $9.82 \mathrm{E}-7$ & +23.4 & 316.7 & +3.5 & 166.5 & $\mathbf{R}$ & 366 \\
\hline $9-5,41-43$ & 76.82 & $1.13 \mathrm{E}-6$ & +4.4 & 334.1 & -14.2 & 158.7 & $\mathbf{R}$ & 28 \\
\hline $9-5,66-68$ & 77.07 & 5.66 E-6 & +15.7 & 345.8 & +19.5 & 352.1 & N & 127 \\
\hline $9-5,90-92$ & 77.31 & 5.56 E-6 & -1.0 & 350.1 & -1.0 & 358.3 & $\mathrm{~N}$ & 124 \\
\hline $9-5,114-116$ & 77.55 & 5.05 E-6 & +4.6 & 356.2 & +11.1 & 358.1 & $\mathrm{~N}$ & 89 \\
\hline $9-5,135-137$ & 77.76 & $5.85 \mathrm{E}-7$ & -3.2 & 211.6 & -0.8 & 174.0 & $\mathbf{R}$ & 462 \\
\hline $9-6,15-17$ & 78.06 & 3.74 E-6 & -3.9 & 351.4 & -10.7 & 355.8 & $\mathrm{~N}$ & 84 \\
\hline $9-6,41-43$ & 78.32 & 3.67 E-6 & +7.4 & 357.6 & +9.9 & 3.3 & N & 184 \\
\hline $9-6,66-68$ & 78.57 & 3.94 E-6 & +1.6 & 1.7 & +5.1 & 7.2 & $\mathrm{~N}$ & 153 \\
\hline $9-6,90-92$ & 78.81 & $3.56 \mathrm{E}-6$ & -0.7 & 6.9 & +4.7 & 13.9 & $N$ & 90 \\
\hline $9-6,114-116$ & 79.05 & 5.43 E-6 & -2.8 & 16.4 & -3.8 & 19.6 & $\mathrm{~N}$ & 177 \\
\hline $9-6,135-137$ & 79.26 & 5.06 E-6 & +0.6 & 15.5 & +3.7 & 17.1 & $\mathrm{~N}$ & 203 \\
\hline $9-7,8-10$ & 79.49 & $6.69 \mathrm{E}-7$ & +4.2 & 351.7 & -13.8 & 220.9 & $\mathbf{R}$ & 35 \\
\hline $9-7,32-34$ & 79.73 & 3.07 E-6 & -4.4 & 23.0 & +1.5 & 24.8 & $\mathrm{~N}$ & 219 \\
\hline $10-1,15-17$ & 80.06 & 2.61 E-6 & -2.3 & 352.1 & -9.9 & 351.7 & $\mathrm{~N}$ & 177 \\
\hline $10-1,41-43$ & 80.32 & 1.44 E-6 & +0.9 & 8.7 & -1.7 & 8.3 & $\mathrm{~N}$ & 198 \\
\hline $10-1,65-67$ & 80.56 & $1.03 \mathrm{E}-6$ & -27.6 & 355.5 & -30.9 & 0.6 & $\mathrm{~N}$ & 228 \\
\hline $10-1,90-92$ & 80.81 & 1.17 E-6 & -29.3 & 353.2 & -31.0 & 0.5 & $\mathrm{~N}$ & 214 \\
\hline $10-1,115-117$ & 81.06 & 1.07 E-6 & -6.2 & 2.3 & -10.1 & 13.4 & $\mathrm{~N}$ & 250 \\
\hline $10-1,141-143$ & 81.32 & $6.12 \mathrm{E}-7$ & -1.4 & 352.6 & -20.2 & 10.5 & $\mathbf{N}$ & 198 \\
\hline $10-2,15-17$ & 81.56 & $1.13 \mathrm{E}-6$ & +8.9 & 357.8 & +4.1 & 1.2 & $\mathrm{~N}$ & 224 \\
\hline $10-2,41-43$ & 81.82 & $1.66 \mathrm{E}-6$ & +17.6 & 60.9 & +28.5 & 24.2 & $\mathrm{~N}$ & 115 \\
\hline $10-2,65-67$ & 82.06 & $6.62 \mathrm{E}-7$ & -21.7 & 354.5 & -12.8 & 360.0 & $N$ & 281 \\
\hline $10-2,90-92$ & 82.31 & 8.09 E-7 & -20.0 & 348.8 & -17.9 & 355.3 & $\mathrm{~N}$ & 282 \\
\hline $10-3,15-17$ & 83.06 & $5.81 \mathrm{E}-7$ & -49.6 & 82.5 & -41.4 & 25.1 & $\mathrm{~N}$ & 195 \\
\hline $10-3,41-43$ & 83.32 & $7.34 \mathrm{E}-7$ & -0.2 & 343.3 & -3.5 & 350.5 & $\mathrm{~N}$ & 230 \\
\hline $10-3,65-67$ & 83.56 & 1.94 E-6 & +1.2 & 329.0 & +3.5 & 331.3 & $\mathrm{~N}$ & 255 \\
\hline $10-3,90-92$ & 83.81 & 4.95 E-6 & +31.4 & 339.0 & +28.9 & 343.3 & N & 203 \\
\hline $10-3,119-121$ & 84.10 & $4.59 \mathrm{E}-6$ & -11.8 & 349.9 & -7.7 & 356.0 & $\mathrm{~N}$ & 199 \\
\hline $10-3,143-145$ & 84.34 & 5.24 E-6 & +15.1 & 342.7 & +15.3 & 345.3 & $N$ & 96 \\
\hline $10-4,15-17$ & 84.56 & $9.25 \mathrm{E}-8$ & +14.1 & 336.4 & no st & able & & \\
\hline $10-4,45-47$ & 84.86 & $1.34 \mathrm{E}-7$ & +8.7 & 352.9 & no st & able & & \\
\hline $10-4,87-89$ & 85.28 & $5.72 \mathrm{E}-8$ & +52.5 & 4.4 & no st & able & & \\
\hline $10-4,117-119$ & 85.58 & $2.18 \mathrm{E}-7$ & +33.5 & 354.3 & no st & able & & \\
\hline $10-5,50-52$ & 86.41 & $1.26 \mathrm{E}-7$ & +81.9 & 321.3 & no st & able & & \\
\hline $10-5,107-109$ & 86.98 & $1.52 \mathrm{E}-7$ & +51.6 & 343.0 & no st & able & & \\
\hline $10-6,18-20$ & 87.59 & $7.61 \mathrm{E}-8$ & -85.1 & 337.4 & no st & able & & \\
\hline $10-6,51-53$ & 87.92 & $5.89 \mathrm{E}-8$ & -52.3 & 311.7 & no st & able & & \\
\hline $10-6,83-85$ & 88.24 & $1.85 \mathrm{E}-7$ & +70.5 & 2.3 & no st & able & & \\
\hline $10-6,118-120$ & 88.59 & $6.76 \mathrm{E}-8$ & +53.2 & 339.6 & no st & able & & \\
\hline $11-1,45-47$ & 89.66 & $1.80 \mathrm{E}-7$ & +25.9 & 14.5 & no st & able & & \\
\hline $11-1,95-97$ & 90.16 & $1.38 \mathrm{E}-7$ & +84.4 & 319.9 & no st & able & & \\
\hline $11-1,115-117$ & 90.36 & $2.83 \mathrm{E}-7$ & -5.6 & 42.0 & no st & able & & \\
\hline $11-2,64-66$ & 91.35 & $1.76 \mathrm{E}-7$ & -15.0 & 64.9 & no st & able & & \\
\hline $11-2,95-97$ & 91.66 & $1.05 \mathrm{E}-7$ & +62.4 & 5.9 & no st & able & & \\
\hline $11-3,95-97$ & 93.16 & $3.46 \mathrm{E}-7$ & +51.7 & 5.8 & no st & able & & \\
\hline $11-3,144-146$ & 93.65 & $1.74 \mathrm{E}-7$ & -1.4 & 42.8 & no st & able & & \\
\hline $11-4,10-12$ & 93.81 & $1.95 \mathrm{E}-7$ & +13.1 & 10.8 & no st & able & & \\
\hline $11-4,95-97$ & 94.66 & $1.35 \mathrm{E}-7$ & +77.5 & 349.5 & no st & able & & \\
\hline $11-5,30-32$ & 95.51 & $1.64 \mathrm{E}-7$ & -14.3 & 42.1 & no st & able & & \\
\hline $11-5,95-97$ & 96.16 & $1.66 \mathrm{E}-7$ & +68.1 & 20.4 & no st & able & & \\
\hline $11-6,95-97$ & 97.66 & $6.17 \mathrm{E}-8$ & -29.5 & 196.9 & no st & able & & \\
\hline $11-6,131-133$ & 98.02 & $9.20 \mathrm{E}-8$ & +64.1 & 80.5 & no st & able & & \\
\hline
\end{tabular}


Appendix A. (Continued).

\begin{tabular}{|c|c|c|c|c|c|c|c|c|}
\hline $\begin{array}{c}\text { Core-Section } \\
\text { (interval in cm) }\end{array}$ & $\begin{array}{l}\text { Sub-bottom } \\
\text { depth } \\
\text { (m) }\end{array}$ & $\begin{array}{c}J_{\text {NRM }} \\
(\mathrm{G})\end{array}$ & $I_{\mathrm{NRM}}$ & $D_{\mathrm{NRM}}$ & $I_{\text {stable }}$ & $D_{\text {stable }}$ & Polarity & $\begin{array}{l}\text { MDF } \\
(\mathrm{Oe})\end{array}$ \\
\hline \multicolumn{9}{|l|}{ Hole 575} \\
\hline $1-1,24-26$ & 0.25 & $1.94 \mathrm{E}-6$ & -25.5 & 48.0 & -9.4 & 17.3 & $\mathrm{~N}$ & 187 \\
\hline $1-1,34-36$ & 0.35 & $4.17 \mathrm{E}-6$ & +26.1 & 21.0 & +14.4 & 28.1 & $\mathrm{~N}$ & 287 \\
\hline $1-1,44-46$ & 0.45 & $4.76 \mathrm{E}-6$ & -5.3 & 15.3 & +7.0 & 27.1 & $\mathrm{~N}$ & 276 \\
\hline $1-1,54-56$ & 0.55 & $7.30 \mathrm{E}-6$ & -5.3 & 39.0 & +4.4 & 35.4 & $\mathrm{~N}$ & 259 \\
\hline $1-1,64-66$ & 0.65 & $4.80 \mathrm{E}-6$ & +22.4 & 21.0 & +14.3 & 28.7 & $\mathrm{~N}$ & 289 \\
\hline $1-1,84-86$ & 0.85 & $4.81 \mathrm{E}-6$ & +29.1 & 29.3 & +17.5 & 27.1 & $\mathrm{~N}$ & 225 \\
\hline $1-1,94-96$ & 0.95 & 7.04 E-6 & +29.0 & 22.6 & +13.5 & 26.3 & $\mathrm{~N}$ & 238 \\
\hline $1-1,104-106$ & 1.05 & $9.05 \mathrm{E}-6$ & +24.9 & 36.0 & +15.2 & 33.0 & $\mathrm{~N}$ & 231 \\
\hline $1-1,114-116$ & 1.15 & 5.35 E-6 & +14.7 & 26.4 & +11.6 & 30.4 & $\mathrm{~N}$ & 254 \\
\hline $1-1,124-126$ & 1.25 & $8.26 \mathrm{E}-6$ & +0.9 & 37.0 & +7.2 & 25.1 & $\mathrm{~N}$ & 156 \\
\hline $1-1,134-136$ & 1.35 & $7.67 \mathrm{E}-6$ & +8.7 & 42.4 & +13.0 & 30.2 & $\mathrm{~N}$ & 185 \\
\hline $1-2,4-6$ & 1.55 & $4.50 \mathrm{E}-6$ & +4.5 & 28.6 & +8.9 & 34.6 & $\mathrm{~N}$ & 254 \\
\hline $1-2,14-16$ & 1.65 & $2.39 \mathrm{E}-6$ & -0.4 & 23.6 & +3.4 & 29.4 & $\mathrm{~N}$ & 260 \\
\hline $1-2,24-26$ & 1.75 & $3.90 \mathrm{E}-6$ & -2.8 & 31.9 & +7.1 & 26.0 & $\mathrm{~N}$ & 227 \\
\hline $1-2,34-36$ & 1.85 & $5.91 \mathrm{E}-6$ & +17.7 & 13.1 & +13.4 & 22.1 & $\mathrm{~N}$ & 254 \\
\hline $1-2,44-46$ & 1.95 & $9.89 \mathrm{E}-6$ & +20.0 & 20.5 & +8.4 & 24.0 & $\mathrm{~N}$ & 225 \\
\hline $1-2,54-56$ & 2.05 & $7.15 \mathrm{E}-6$ & +3.1 & 13.9 & +12.5 & 20.7 & $\mathrm{~N}$ & 238 \\
\hline $1-2,64-66$ & 2.15 & $4.36 \mathrm{E}-6$ & -1.8 & 30.8 & +6.9 & 24.9 & $\mathrm{~N}$ & 194 \\
\hline $1-2,84-86$ & 2.35 & $9.35 \mathrm{E}-6$ & +14.9 & 11.4 & +16.0 & 20.8 & $\mathrm{~N}$ & 253 \\
\hline $1-2,94-96$ & 2.45 & 8.20 E-6 & -6.1 & 20.8 & +7.4 & 20.9 & $\mathrm{~N}$ & 174 \\
\hline $1-2,114-116$ & 2.65 & $4.08 \mathrm{E}-6$ & +21.2 & 23.7 & +51.4 & 10.7 & $\mathrm{~N}$ & 45 \\
\hline $1-2,124-126$ & 2.75 & $4.06 \mathrm{E}-6$ & +13.6 & 150.6 & -7.9 & 173.5 & $\mathrm{R}$ & 353 \\
\hline $1-2,134-136$ & 2.85 & $2.61 \mathrm{E}-6$ & -43.5 & 140.8 & -21.8 & 177.1 & $\mathbf{R}$ & 322 \\
\hline $1-2,144-146$ & 2.95 & $2.49 \mathrm{E}-6$ & -32.2 & 243.4 & -16.6 & 182.1 & $\mathbf{R}$ & 315 \\
\hline $1-3,4-6$ & 3.05 & 2.95 E-6 & +43.5 & 299.1 & +4.0 & 215.8 & $\mathbf{R}$ & 33 \\
\hline $1-3,14-16$ & 3.15 & 2.74 E-6 & -13.7 & 3.1 & +35.4 & 357.8 & $\mathrm{~N}$ & 57 \\
\hline $1-3,24-26$ & 3.25 & $2.26 \mathrm{E}-6$ & +34.2 & 228.7 & -4.6 & 188.8 & $\mathbf{R}$ & 323 \\
\hline $1-3,34-36$ & 3.35 & $1.85 \mathrm{E}-6$ & +26.6 & 263.4 & -0.4 & 192.5 & $R$ & 256 \\
\hline $1-3,44-46$ & 3.45 & 2.46 E- 6 & +40.3 & 267.8 & +0.1 & 188.4 & $\mathrm{R}$ & 267 \\
\hline $1-3,54-56$ & 3.55 & 3.16 E-6 & -41.0 & 137.5 & -12.2 & 177.2 & $\mathrm{R}$ & 305 \\
\hline $1-3,64-66$ & 3.65 & 2.34 E-6 & -11.0 & 218.6 & -12.3 & 180.4 & R & 373 \\
\hline $1-3,84-86$ & 3.85 & $2.18 \mathrm{E}-6$ & -8.8 & 201.2 & -7.0 & 178.9 & R & 407 \\
\hline $1-3,94-96$ & 3.95 & $1.93 \mathrm{E}-6$ & -20.4 & 209.7 & -15.9 & 176.1 & R & 380 \\
\hline $1-3,104-106$ & 4.05 & $1.93 \mathrm{E}-6$ & -23.1 & 140.1 & -8.9 & 167.0 & $\mathrm{R}$ & 350 \\
\hline $1-3,114-116$ & 4.15 & $4.75 \mathrm{E}-6$ & +15.9 & 312.5 & +35.1 & 331.8 & $\mathrm{~N}$ & 43 \\
\hline $1-3,124-126$ & 4.25 & $3.01 \mathrm{E}-6$ & +33.9 & 33.9 & -65.0 & 10.2 & $\mathrm{~N}$ & 36 \\
\hline $1-3,134-136$ & 4.35 & $1.52 \mathrm{E}-6$ & -59.8 & 220.0 & -6.4 & 168.2 & R & 435 \\
\hline $1-3,144-146$ & 4.45 & $2.01 \mathrm{E}-6$ & -48.5 & 250.9 & -14.7 & 184.0 & $\mathbf{R}$ & 301 \\
\hline $1-4,4-6$ & 4.55 & $1.98 \mathrm{E}-6$ & -30.1 & 173.5 & -9.1 & 169.4 & $\mathbf{R}$ & 345 \\
\hline $1-4,14-16$ & 4.65 & $5.91 \mathrm{E}-7$ & +19.7 & 181.2 & +5.8 & 165.5 & $\mathbf{R}$ & 401 \\
\hline $1-4,24-26$ & 4.75 & $9.48 \mathrm{E}-7$ & -51.5 & 235.4 & -12.0 & 171.4 & $\mathrm{R}$ & 371 \\
\hline $1-4,34-36$ & 4.85 & $2.16 \mathrm{E}-6$ & +8.8 & 191.9 & -0.3 & 167.6 & $\mathbf{R}$ & 405 \\
\hline $1-4,44-46$ & 4.95 & $1.52 \mathrm{E}-6$ & +32.0 & 249.5 & +5.8 & 169.3 & $\mathrm{R}$ & 360 \\
\hline $1-4,55-57$ & 5.06 & $1.95 \mathrm{E}-6$ & -1.6 & 198.3 & -14.6 & 159.7 & $\mathbf{R}$ & 406 \\
\hline $1-4,64-66$ & 5.15 & $2.54 \mathrm{E}-6$ & -29.7 & 148.3 & -11.4 & 156.9 & $\mathbf{R}$ & 344 \\
\hline $1-4,84-86$ & 5.35 & $5.58 \mathrm{E}-7$ & +18.5 & 207.5 & -4.9 & 171.4 & $\mathrm{R}$ & 357 \\
\hline $1-4,94-96$ & 5.45 & 9.33 E-7 & +4.5 & 4.2 & +17.5 & 339.1 & $\mathrm{~N}$ & 142 \\
\hline $1-4,104-106$ & 5.55 & $2.39 \mathrm{E}-6$ & -7.3 & 344.6 & +3.9 & 340.8 & $\mathrm{~N}$ & 161 \\
\hline $1-4,114-116$ & 5.65 & $1.51 \mathrm{E}-6$ & -11.2 & 351.1 & +7.4 & 340.0 & $\mathrm{~N}$ & 155 \\
\hline $1-4,124-126$ & 5.75 & $1.69 \mathrm{E}-6$ & -9.8 & 328.2 & +0.4 & 335.2 & $\mathrm{~N}$ & 87 \\
\hline $1-4,134-136$ & 5.85 & $6.39 \mathrm{E}-6$ & +14.4 & 320.5 & +5.8 & 330.1 & $\mathrm{~N}$ & 93 \\
\hline $1-4,144-146$ & 5.95 & $7.86 \mathrm{E}-6$ & -5.7 & 315.7 & -1.1 & 327.1 & $\mathrm{~N}$ & 76 \\
\hline $1-5,4-6$ & 6.05 & $6.62 \mathrm{E}-6$ & -6.8 & 352.2 & +4.5 & 343.9 & $\mathrm{~N}$ & 197 \\
\hline $1-5,14-16$ & 6.15 & $9.01 \mathrm{E}-6$ & +28.0 & 319.9 & +18.6 & 336.1 & $\mathrm{~N}$ & 79 \\
\hline $1-5,24-26$ & 6.25 & $8.78 \mathrm{E}-6$ & +26.5 & 329.9 & +14.2 & 338.0 & $\mathrm{~N}$ & 125 \\
\hline $1-5,34-36$ & 6.35 & $8.26 \mathrm{E}-6$ & -3.9 & 327.3 & +4.5 & 336.3 & $\mathrm{~N}$ & 164 \\
\hline $1-5,44-46$ & 6.45 & $9.10 \mathrm{E}-6$ & +17.2 & 319.8 & +11.6 & 328.4 & $\mathrm{~N}$ & 130 \\
\hline $1-5,54-56$ & 6.55 & $4.68 \mathrm{E}-6$ & -18.3 & 336.3 & +5.1 & 329.6 & $\mathrm{~N}$ & 86 \\
\hline $1-5,64-66$ & 6.65 & $3.14 \mathrm{E}-6$ & +29.0 & 304.1 & +10.3 & 203.3 & $\mathrm{R}$ & 30 \\
\hline $1-5,84-86$ & 6.85 & $8.66 \mathrm{E}-7$ & +46.4 & 284.7 & -1.6 & 158.9 & $\mathrm{R}$ & 33 \\
\hline $1-5,94-96$ & 6.95 & $3.75 \mathrm{E}-6$ & -12.8 & 343.0 & +4.9 & 333.9 & $\mathrm{~N}$ & 155 \\
\hline $1-5,104-106$ & 7.05 & $5.17 \mathrm{E}-6$ & -11.4 & 340.2 & -1.9 & 336.3 & $\mathrm{~N}$ & 153 \\
\hline $1-5,114-116$ & 7.15 & $3.23 \mathrm{E}-6$ & -2.4 & 328.2 & +6.4 & 328.5 & $\mathrm{~N}$ & 137 \\
\hline $1-5,122-124$ & 7.23 & 4.32 E-6 & +15.0 & 220.0 & +7.7 & 206.3 & R & 107 \\
\hline $1-5,127-129$ & 7.28 & 2.35 E-6 & -4.9 & 209.0 & -7.4 & 211.4 & R & 46 \\
\hline $2-1,3-5$ & 7.34 & $3.25 \mathrm{E}-6$ & -84.3 & 39.1 & -77.3 & 12.4 & $\mathrm{~N}$ & 267 \\
\hline $2-1,19-21$ & 7.50 & $3.25 \mathrm{E}-6$ & -44.8 & 282.4 & -68.9 & 296.8 & $\mathrm{~N}$ & 44 \\
\hline $2-1,29-31$ & 7.60 & 1.85 E-6 & -37.4 & 54.8 & -45.5 & 350.9 & $\mathrm{~N}$ & 37 \\
\hline $2-1,39-41$ & 7.70 & 2.67 E-6 & +51.4 & 21.3 & -63.8 & 152.5 & R & 30 \\
\hline $2-1,49-51$ & 7.80 & $6.81 \mathrm{E}-6$ & -21.6 & 276.3 & -13.2 & 190.4 & $\mathrm{R}$ & 39 \\
\hline $2-1,59-61$ & 7.90 & $3.56 \mathrm{E}-6$ & +20.7 & 288.8 & -10.3 & 209.7 & $\mathrm{R}$ & 24 \\
\hline $2-1,69-71$ & 8.00 & 2.95 E-6 & +40.5 & 73.4 & -11.8 & 158.9 & $\mathrm{R}$ & 45 \\
\hline $2-1,79-81$ & 8.10 & $3.11 \mathrm{E}-6$ & -5.3 & 64.7 & -22.4 & 153.2 & R & 39 \\
\hline
\end{tabular}


Appendix A. (Continued).

\begin{tabular}{|c|c|c|c|c|c|c|c|c|}
\hline $\begin{array}{l}\text { Core-Section } \\
\text { (interval in } \mathrm{cm} \text { ) }\end{array}$ & $\begin{array}{l}\text { Sub-bottom } \\
\text { depth } \\
\text { (m) }\end{array}$ & $\begin{array}{c}J_{\text {NRM }} \\
(\mathrm{G})\end{array}$ & $I_{\mathrm{NRM}}$ & $D_{\mathrm{NRM}}$ & $I_{\text {stable }}$ & $D_{\text {stable }}$ & Polarity & $\begin{array}{l}\text { MDF } \\
\text { (Oe) }\end{array}$ \\
\hline \multicolumn{9}{|l|}{ Hole 575 (Cont.) } \\
\hline $2-1,89-91$ & 8.20 & $3.45 \mathrm{E}-6$ & -66.6 & 1.4 & -45.3 & 166.2 & $\mathbf{R}$ & 23 \\
\hline $2-1,99-101$ & 8.30 & $3.65 \mathrm{E}-6$ & +33.8 & 286.3 & -3.0 & 187.7 & $\mathbf{R}$ & 45 \\
\hline $2-1,109-111$ & 8.40 & $3.65 \mathrm{E}-6$ & +66.6 & 307.5 & -0.8 & 176.4 & $\mathbf{R}$ & 39 \\
\hline $2-1,119-121$ & 8.50 & 3.47 E-6 & +6.3 & 95.3 & -7.2 & 168.1 & $\mathbf{R}$ & 250 \\
\hline $2-1,129-131$ & 8.60 & 3.41 E-6 & -56.7 & 291.7 & -31.9 & 192.0 & $\mathbf{R}$ & 44 \\
\hline $2-1,140-142$ & 8.71 & $2.34 \mathrm{E}-6$ & +29.4 & 62.7 & -23.2 & 168.1 & $\mathbf{R}$ & 41 \\
\hline $2-2,19-21$ & 9.00 & $3.33 \mathrm{E}-6$ & -21.3 & 260.8 & -4.4 & 186.8 & $\mathbf{R}$ & 246 \\
\hline $2-2,29-31$ & 9.10 & 3.23 E-6 & -13.0 & 69.1 & -16.3 & 162.3 & $\mathbf{R}$ & 274 \\
\hline $2-2,39-41$ & 9.20 & $3.20 \mathrm{E}-6$ & -18.2 & 66.4 & -18.6 & 162.3 & $\mathbf{R}$ & 43 \\
\hline $2-2,49-51$ & 9.30 & $4.38 \mathrm{E}-6$ & -75.4 & 0.9 & -31.5 & 167.7 & $\mathrm{R}$ & 45 \\
\hline $2-2,59-61$ & 9.40 & 4.03 E-6 & -58.3 & 80.1 & -22.7 & 169.6 & $\mathbf{R}$ & 228 \\
\hline $2-2,69-71$ & 9.50 & 2.07 E-6 & -48.3 & 334.6 & -17.4 & 185.5 & $\mathbf{R}$ & 286 \\
\hline $2-2,79-81$ & 9.60 & 3.39 E-6 & +36.8 & 291.2 & -4.9 & 190.6 & R & 243 \\
\hline $2-2,89-91$ & 9.70 & 4.54 E-6 & +24.0 & 297.2 & -7.5 & 199.8 & $\mathbf{R}$ & 35 \\
\hline $2-2,99-101$ & 9.80 & 3.86 E-6 & -53.8 & 41.5 & -33.5 & 180.2 & $\mathrm{R}$ & 33 \\
\hline $2-2,109-111$ & 9.90 & 3.78 E-6 & -63.9 & 43.6 & -28.9 & 178.5 & $\mathbf{R}$ & 39 \\
\hline $2-2,119-121$ & 10.00 & 4.97 E-6 & +56.3 & 315.5 & -8.3 & 195.8 & R & 29 \\
\hline $2-2,129-131$ & 10.10 & $4.68 \mathrm{E}-6$ & +37.3 & 304.0 & -10.9 & 223.6 & $\mathbf{R}$ & 30 \\
\hline $2-2,139-141$ & 10.20 & 5.85 E-6 & +11.9 & 81.0 & -26.2 & 156.8 & $\mathbf{R}$ & 32 \\
\hline $2-3,3-5$ & 10.34 & 4.44 E-6 & +29.6 & 11.9 & +28.4 & 10.7 & $\mathrm{~N}$ & 38 \\
\hline $2-3,20-22$ & 10.51 & $3.25 \mathrm{E}-6$ & +0.7 & 2.2 & +4.4 & 6.3 & $\mathrm{~N}$ & 83 \\
\hline $2-3,29-31$ & 10.60 & 5.20 E-6 & +52.6 & 315.1 & +29.8 & 344.6 & $\mathbf{N}$ & 44 \\
\hline $2-3,41-43$ & 10.72 & $3.53 \mathrm{E}-6$ & +60.3 & 3.5 & +14.4 & 349.3 & $\mathbf{N}$ & 38 \\
\hline $2-3,59-61$ & 10.90 & 4.00 E- 6 & +38.2 & 31.8 & +21.7 & 8.6 & $\mathrm{~N}$ & 50 \\
\hline $2-3,69-71$ & 11.00 & 2.96 E-6 & +11.1 & 305.8 & -26.2 & 328.3 & $\mathrm{~N}$ & 36 \\
\hline $2-3,82-84$ & 11.13 & 3.79 E-6 & +16.5 & 304.5 & -4.5 & 333.3 & $\mathrm{~N}$ & 36 \\
\hline $2-3,89-91$ & 11.20 & 3.84 E-6 & -2.7 & 60.6 & +2.0 & 11.4 & $\mathrm{~N}$ & 42 \\
\hline $2-3,99-101$ & 11.30 & 4.72 E-6 & +42.2 & 317.4 & +30.5 & 347.1 & $\mathrm{~N}$ & 46 \\
\hline $2-3,109-111$ & 11.40 & 5.21 E-6 & +66.6 & 10.2 & +29.0 & 353.0 & $\mathrm{~N}$ & 38 \\
\hline $2-3,119-121$ & 11.50 & 3.87 E-6 & +21.9 & 315.1 & +11.3 & 347.2 & $\mathrm{~N}$ & 42 \\
\hline $2-3,129-131$ & 11.60 & 5.15 E-6 & +51.8 & 319.4 & +23.1 & 350.3 & $\mathrm{~N}$ & 37 \\
\hline $2-3,146-148$ & 11.77 & 4.12 E-6 & +14.4 & 350.2 & +20.3 & 5.0 & $\mathrm{~N}$ & 69 \\
\hline $2-4,25-27$ & 12.06 & 4.95 E-6 & +18.8 & 329.9 & +6.1 & 350.9 & $\mathrm{~N}$ & 52 \\
\hline $2-4,87-89$ & 12.68 & 3.17 E-6 & +13.4 & 0.4 & -20.1 & 4.7 & $\mathrm{~N}$ & 73 \\
\hline $2-4,127-129$ & 13.08 & 6.37 E-6 & -62.1 & 305.4 & -49.7 & 308.5 & $\mathrm{~N}$ & 402 \\
\hline $2-4,144-146$ & 13.25 & 2.34 E-6 & +22.7 & 321.2 & +2.7 & 245.2 & $\mathbf{R}$ & 32 \\
\hline $2-5,9-11$ & 13.40 & $1.26 \mathrm{E}-6$ & +7.4 & 325.4 & -23.3 & 235.0 & $\mathbf{R}$ & 45 \\
\hline $2-5,19-21$ & 13.50 & $1.28 \mathrm{E}-6$ & -13.3 & 310.8 & -16.7 & 227.6 & $\mathbf{R}$ & 47 \\
\hline $2-5,29-31$ & 13.60 & 1.19 E-6 & -1.9 & 316.0 & -5.4 & 230.4 & $\mathbf{R}$ & 322 \\
\hline $2-5,39-41$ & 13.70 & 2.38 E-6 & -58.7 & 77.5 & -20.7 & 207.8 & R & 252 \\
\hline $2-5,49-51$ & 13.80 & 2.00 E-6 & +52.8 & 353.3 & +3.7 & 252.0 & $\mathbf{R}$ & 29 \\
\hline $2-5,59-61$ & 13.90 & 1.28 E- 6 & +8.1 & 335.8 & -12.2 & 242.8 & $\mathbf{R}$ & 36 \\
\hline $2-5,69-71$ & 14.00 & 2.01 E-6 & +12.8 & 276.8 & -8.3 & 221.8 & $\mathbf{R}$ & 303 \\
\hline $2-5,79-81$ & 14.10 & 1.48 E-6 & -38.7 & 307.9 & -25.2 & 221.7 & $\mathbf{R}$ & 331 \\
\hline $2-5,89-91$ & 14.20 & 2.09 E-6 & +55.8 & 344.2 & +17.1 & 218.2 & $\mathbf{R}$ & 31 \\
\hline $2-5,99-101$ & 14.30 & 1.94 E-6 & +61.6 & 284.3 & +4.3 & 215.6 & $\mathrm{R}$ & 46 \\
\hline $2-5,109-111$ & 14.40 & 1.79 E-6 & -23.6 & 330.9 & -8.8 & 235.4 & $\mathbf{R}$ & 29 \\
\hline $2-5,119-121$ & 14.50 & 3.01 E-6 & +53.2 & 302.3 & +7.0 & 224.3 & $\mathbf{R}$ & 34 \\
\hline $2-5,129-131$ & 14.60 & 1.70 E-6 & +63.1 & 11.8 & -1.8 & 208.5 & $\mathbf{R}$ & 42 \\
\hline $2-5,139-141$ & 14.70 & $1.19 \mathrm{E}-6$ & -40.7 & 97.7 & -13.7 & 187.1 & $\mathbf{R}$ & 343 \\
\hline $2-6,9-11$ & 14.90 & $1.39 \mathrm{E}-6$ & +9.4 & 264.5 & -14.3 & 204.1 & $\mathbf{R}$ & 49 \\
\hline $2-6,19-21$ & 15.00 & 1.74 E-6 & -38.9 & 257.3 & -24.0 & 203.9 & $\mathbf{R}$ & 282 \\
\hline $2-6,29-31$ & 15.10 & $7.88 \mathrm{E}-7$ & +25.2 & 318.8 & -29.7 & 262.1 & $\mathbf{R}$ & 38 \\
\hline $2-6,39-41$ & 15.20 & 8.46 E-7 & -32.8 & 99.0 & -30.6 & 183.5 & $\mathbf{R}$ & 269 \\
\hline $2-6,49-51$ & 15.30 & $9.38 \mathrm{E}-7$ & -19.5 & 318.5 & -38.0 & 224.8 & $\mathrm{~N}$ & 49 \\
\hline $2-6,59-61$ & 15.40 & $4.57 \mathrm{E}-7$ & -40.4 & 51.2 & -55.6 & 210.3 & $\mathrm{~N}$ & 39 \\
\hline $2-6,69-71$ & 15.50 & $5.63 \mathrm{E}-7$ & -73.5 & 30.7 & -50.5 & 209.5 & $\mathrm{~N}$ & 40 \\
\hline $2-6,79-81$ & 15.60 & $3.73 \mathrm{E}-7$ & +33.1 & 279.9 & +1.7 & 207.4 & $\mathbf{R}$ & 306 \\
\hline $2-6,89-91$ & 15.70 & $6.53 \mathrm{E}-7$ & +66.7 & 265.8 & +6.8 & 198.3 & $R$ & 258 \\
\hline $2-6,99-101$ & 15.80 & 8.37 E-7 & +88.5 & 58.1 & +5.4 & 202.8 & R & 317 \\
\hline $2-6,109-111$ & 15.90 & 1.00 E-6 & -69.2 & 338.8 & -36.7 & 194.2 & $\mathrm{R}$ & 35 \\
\hline $2-6,119-121$ & 16.00 & 8.54 E-7 & +0.4 & 292.0 & -27.0 & 215.3 & $\mathbf{R}$ & 38 \\
\hline $2-6,129-131$ & 16.10 & 4.44 E-7 & -19.3 & 12.6 & -37.9 & 208.8 & $\mathbf{R}$ & 42 \\
\hline $2-6,139-141$ & 16.20 & 8.01 E-7 & -42.8 & 41.9 & -62.3 & 359.6 & $\mathrm{~N}$ & 40 \\
\hline $2-7,5-7$ & 16.36 & 1.04 E-6 & +23.8 & 347.0 & -12.7 & 339.7 & $\mathrm{~N}$ & 50 \\
\hline $2-7,16-18$ & 16.47 & $1.39 \mathrm{E}-6$ & -41.9 & 336.9 & -30.2 & 342.0 & $\mathrm{~N}$ & 50 \\
\hline $2-7,25-27$ & 16.56 & 1.95 E-6 & -42.5 & 353.4 & -33.7 & 351.7 & $\mathrm{~N}$ & 66 \\
\hline $2-7,36-38$ & 16.67 & 8.27 E-7 & -33.7 & 349.2 & -20.0 & 329.7 & $N$ & 43 \\
\hline $2-7,45-47$ & 16.76 & 1.24 E-6 & -12.3 & 322.7 & -28.2 & 332.0 & $\mathrm{~N}$ & 41 \\
\hline $2-7,49-51$ & 16.80 & $1.27 \mathrm{E}-6$ & +57.9 & 0.7 & +27.7 & 291.5 & $\mathrm{~N}$ & 142 \\
\hline $3-1,9-11$ & 16.90 & $1.35 \mathrm{E}-6$ & -78.6 & 82.8 & -80.8 & 31.2 & $\mathrm{~N}$ & 49 \\
\hline $3-1,19-21$ & 17.00 & $1.76 \mathrm{E}-6$ & -58.0 & 55.2 & -59.9 & 57.4 & $\mathrm{~N}$ & 62 \\
\hline $3-1,29-31$ & 17.10 & 9.40 E-7 & -70.3 & 36.1 & -69.0 & 35.9 & $\mathrm{~N}$ & 74 \\
\hline
\end{tabular}


Appendix A. (Continued).

\begin{tabular}{|c|c|c|c|c|c|c|c|c|}
\hline $\begin{array}{c}\text { Core-Section } \\
\text { (interval in cm) }\end{array}$ & $\begin{array}{l}\text { Sub-bottom } \\
\text { depth } \\
\text { (m) }\end{array}$ & $\begin{array}{l}J_{\text {NRM }} \\
(\mathrm{G})\end{array}$ & $I_{\mathrm{NRM}}$ & $D_{\mathrm{NRM}}$ & $I_{\text {stable }}$ & $D_{\text {stable }}$ & Polarity & $\begin{array}{l}\text { MDF } \\
(\mathrm{Oe})\end{array}$ \\
\hline \multicolumn{9}{|l|}{ Hole 575 (Cont.) } \\
\hline $3-1,79-81$ & 17.60 & $5.24 \mathrm{E}-7$ & -11.4 & 54.3 & -63.6 & 117.9 & $\mathrm{~N}$ & 203 \\
\hline $3-1,89-91$ & 17.70 & $1.30 \mathrm{E}-6$ & $-30,3$ & 61.3 & -42.1 & 64.1 & $\mathrm{~N}$ & 146 \\
\hline $3-1,99-101$ & 17.80 & $1.66 \mathrm{E}-6$ & -23.5 & 279.7 & -59.1 & 172.2 & $\mathrm{~N}$ & 208 \\
\hline $3-1,109-111$ & 17.90 & $3.39 \mathrm{E}-6$ & -54.2 & 350.6 & -56.7 & 50.6 & $\mathrm{~N}$ & 46 \\
\hline $3-1,119-121$ & 18.00 & $2.43 \mathrm{E}-6$ & -70.5 & 47.1 & -71.3 & 93.1 & $\mathrm{~N}$ & 117 \\
\hline $3-2,19-21$ & 18.50 & $2.63 \mathrm{E}-6$ & -50.4 & 25.0 & -59.1 & 24.7 & $\mathrm{~N}$ & 45 \\
\hline $3-2,29-31$ & 18.60 & $2.57 \mathrm{E}-6$ & -19.0 & 43.8 & -71.5 & 87.0 & $\mathrm{~N}$ & 44 \\
\hline $3-2,79-81$ & 19.10 & $3.12 \mathrm{E}-6$ & -42.0 & 41.0 & -27.9 & 41.4 & $\mathrm{~N}$ & 45 \\
\hline $3-2,99-101$ & 19.30 & $2.71 \mathrm{E}-6$ & -31.4 & 46.0 & -40.4 & 29.4 & $\mathrm{~N}$ & 73 \\
\hline $3-2,129-131$ & 19.60 & $1.21 \mathrm{E}-6$ & +32.0 & 344.2 & -41.5 & 38.3 & $\mathrm{~N}$ & 299 \\
\hline $3-2,139-141$ & 19.70 & $2.03 \mathrm{E}-6$ & +52.2 & 336.3 & -35.7 & 24.3 & $\mathrm{~N}$ & 42 \\
\hline $3-3,29-31$ & 20.10 & $3.03 \mathrm{E}-6$ & -26.1 & 21.8 & -54.1 & 0.9 & $\mathrm{~N}$ & 83 \\
\hline $3-3,39-41$ & 20.20 & $9.58 \mathrm{E}-7$ & -19.3 & 20.2 & -50.3 & 61.9 & $\mathrm{~N}$ & 292 \\
\hline $3-3,59-61$ & 20.40 & $2.25 \mathrm{E}-6$ & -31.0 & 298.9 & -73.5 & 10.6 & $\mathrm{~N}$ & 94 \\
\hline $3-3,69-71$ & 20.50 & $1.48 \mathrm{E}-6$ & -3.4 & 289.0 & -45.0 & 360.0 & $\mathrm{~N}$ & 190 \\
\hline $3-3,79-81$ & 20.60 & $2.12 \mathrm{E}-6$ & +34.3 & 8.6 & +8.5 & 49.4 & $\mathrm{~N}$ & 41 \\
\hline $3-3,89-91$ & 20.70 & $2.27 \mathrm{E}-6$ & +47.5 & 41.6 & +10.9 & 38.8 & $\mathrm{~N}$ & 45 \\
\hline $3-3,99-101$ & 20.80 & $1.48 \mathrm{E}-6$ & -50.6 & 319.1 & -52.4 & 13.9 & $\mathrm{~N}$ & 61 \\
\hline $3-3,109-111$ & 20.90 & $1.19 \mathrm{E}-6$ & -42.5 & 54.4 & -45.3 & 34.4 & $\mathrm{~N}$ & 85 \\
\hline $3-3,119-121$ & 21.00 & $1.52 \mathrm{E}-6$ & -45.3 & 43.4 & -51.9 & 59.5 & $\mathrm{~N}$ & 72 \\
\hline $3-3,129-131$ & 21.10 & $5.68 \mathrm{E}-7$ & +24.7 & 26.8 & -61.1 & 44.1 & $\mathrm{~N}$ & 268 \\
\hline $3-3,139-141$ & 21.20 & 1.79 E-6 & -59.5 & 37.2 & -38.6 & 49.7 & $\mathrm{~N}$ & 50 \\
\hline $3-4,29-31$ & 21.60 & $2.00 \mathrm{E}-6$ & -69.4 & 39.1 & -40.8 & 34.7 & $\mathrm{~N}$ & 95 \\
\hline $3-4,49-51$ & 21.80 & 3.01 E-7 & -11.4 & 99.1 & -56.2 & 63.3 & $\mathrm{~N}$ & 546 \\
\hline $3-4,59-61$ & 21.90 & $1.93 \mathrm{E}-6$ & -46.9 & 42.8 & -64.2 & 52.2 & $\mathrm{~N}$ & 90 \\
\hline $3-4,69-71$ & 22.00 & 7.68 E-7 & -71.8 & 211.9 & -50.4 & 80.5 & $\mathrm{~N}$ & 286 \\
\hline $3-4,79-81$ & 22.10 & $2.01 \mathrm{E}-7$ & -75.5 & 87.4 & -31.8 & 63.4 & $\mathrm{~N}$ & 549 \\
\hline $3-4,99-101$ & 22.30 & $1.95 \mathrm{E}-6$ & -26.7 & 86.8 & -26.8 & 98.8 & $\mathrm{~N}$ & 58 \\
\hline $3-4,109-111$ & 22.40 & 2.04 E-6 & -50.9 & 59.1 & -44.2 & 59.2 & $\mathrm{~N}$ & 48 \\
\hline $3-4,138-140$ & 22.69 & $7.13 \mathrm{E}-7$ & +45.3 & 70.2 & +72.5 & 22.7 & $\mathrm{~N}$ & 187 \\
\hline $3-5,4-6$ & 22.85 & $1.84 \mathrm{E}-6$ & +47.8 & 176.0 & +47.8 & 176.0 & $\mathrm{R}$ & 42 \\
\hline $3-5,89-91$ & 23.70 & $5.16 \mathrm{E}-7$ & +83.7 & 179.0 & -22.5 & 150.0 & $\mathbf{R}$ & 41 \\
\hline $3-5,107-109$ & 23.88 & $1.42 \mathrm{E}-7$ & +30.1 & 317.7 & -4.9 & 105.3 & $\mathrm{R}$ & 376 \\
\hline $3-5,123-125$ & 24.04 & $1.10 \mathrm{E}-6$ & +82.0 & 83.2 & +47.7 & 120.7 & $\mathrm{R}$ & 41 \\
\hline $3-5,139-141$ & 24.20 & $1.10 \mathrm{E}-6$ & -51.4 & 45.9 & -27.5 & 105.5 & $\mathbf{R}$ & 38 \\
\hline $3-6,9-11$ & 24.40 & $1.52 \mathrm{E}-6$ & -14.7 & 62.1 & -18.3 & 169.2 & $\mathrm{R}$ & 35 \\
\hline $3-6,19-21$ & 24.50 & $6.28 \mathrm{E}-7$ & -19.4 & 53.0 & -1.6 & 171.6 & $\mathbf{R}$ & 36 \\
\hline $3-6,29-31$ & 24.60 & $1.33 \mathrm{E}-6$ & +49.4 & 43.5 & +37.1 & 130.4 & $\mathrm{R}$ & 43 \\
\hline $3-6,39-41$ & 24.70 & $6.92 \mathrm{E}-7$ & +64.9 & 289.2 & -5.2 & 181.0 & $\mathrm{R}$ & 40 \\
\hline $3-6,49-51$ & 24.80 & $1.39 \mathrm{E}-6$ & -30.0 & 48.8 & -29.2 & 152.1 & $R$ & 33 \\
\hline $3-6,59-61$ & 24.90 & $9.39 \mathrm{E}-7$ & +89.3 & 28.2 & +6.3 & 202.6 & $\mathbf{R}$ & 143 \\
\hline $3-6,69-71$ & 25.00 & $1.02 \mathrm{E}-6$ & +42.2 & 78.3 & +3.2 & 179.3 & $\mathbf{R}$ & 39 \\
\hline $3-6,79-81$ & 25.10 & $9.72 \mathrm{E}-7$ & +26.6 & 65.9 & +11.6 & 146.8 & R & 35 \\
\hline $3-6,89-91$ & 25.20 & $1.26 \mathrm{E}-6$ & +58.3 & 235.5 & +6.6 & 180.3 & $\mathbf{R}$ & 41 \\
\hline $3-6,99-101$ & 25.30 & $1.56 \mathrm{E}-6$ & -55.8 & 73.2 & -3.9 & 192.0 & $\mathbf{R}$ & 258 \\
\hline $3-6,109-111$ & 25.40 & $6.86 \mathrm{E}-7$ & +44.3 & 240.5 & -15.7 & 177.8 & $\mathrm{R}$ & 46 \\
\hline $3-6,119-121$ & 25.50 & $1.43 \mathrm{E}-6$ & -28.5 & 64.8 & -28.4 & 182.9 & R & 42 \\
\hline $3-6,129-131$ & 25.60 & $1.45 \mathrm{E}-6$ & -25.0 & 70.5 & -36.1 & 185.3 & $\mathrm{R}$ & 259 \\
\hline $3-6,139-141$ & 25.70 & $1.93 \mathrm{E}-6$ & -4.3 & 71.5 & -13.4 & 187.5 & $\ddot{R}$ & 278 \\
\hline $3-7,9-11$ & 25.90 & $4.79 \mathrm{E}-6$ & -5.0 & 50.3 & -47.8 & 51.7 & $\mathrm{~N}$ & 37 \\
\hline $3-7,19-21$ & 26.00 & $3.07 \mathrm{E}-6$ & +27.7 & 57.1 & -22.7 & 159.8 & $\mathbf{R}$ & 32 \\
\hline $3-7,29-31$ & 26.10 & $2.36 \mathrm{E}-6$ & -16.4 & 73.1 & -3.4 & 203.7 & $\mathbf{R}$ & 40 \\
\hline $3-7,39-41$ & 26.20 & $1.76 \mathrm{E}-6$ & -71.8 & 101.8 & -18.8 & 213.0 & R & 310 \\
\hline $4-1,9-11$ & 26.40 & 2.75 E-6 & -37.7 & 345.0 & -19.8 & 199.8 & $\mathbf{R}$ & 93 \\
\hline $4-1,18-20$ & 26.49 & $1.38 \mathrm{E}-6$ & -25.7 & 221.8 & -23.8 & 168.0 & $\mathrm{R}$ & 428 \\
\hline $4-1,29-31$ & 26.60 & $2.22 \mathrm{E}-6$ & +38.9 & 355.7 & +44.9 & 192.1 & R & 832 \\
\hline $4-1,38-40$ & 26.69 & $2.50 \mathrm{E}-6$ & -70.7 & 44.2 & -7.8 & 190.9 & $\mathbf{R}$ & 41 \\
\hline $4-1,49-51$ & 26.80 & $2.94 \mathrm{E}-6$ & +49.9 & 19.0 & +1.8 & 188.2 & $\mathrm{R}$ & 36 \\
\hline $4-1,59-61$ & 26.90 & $4.99 \mathrm{E}-6$ & +24.4 & 322.1 & +17.2 & 322.4 & $\mathrm{~N}$ & 40 \\
\hline $4-1,69-71$ & 27.00 & $3.56 \mathrm{E}-6$ & -14.2 & 318.6 & +30.0 & 330.1 & $\mathrm{~N}$ & 47 \\
\hline $4-1,90-92$ & 27.21 & $5.94 \mathrm{E}-6$ & -13.9 & 142.3 & -18.8 & 124.6 & $\mathrm{R}$ & 47 \\
\hline $4-1,110-112$ & 27.41 & $3.21 \mathrm{E}-6$ & -5.5 & 158.9 & -6.2 & 153.8 & $\mathrm{R}$ & 67 \\
\hline $4-1,130-132$ & 27.61 & $3.96 \mathrm{E}-6$ & +8.5 & 159.4 & -11.9 & 173.4 & $\mathrm{R}$ & 91 \\
\hline $4-1,145-147$ & 27.76 & $3.61 \mathrm{E}-6$ & -7.1 & 150.1 & -13.6 & 145.9 & $\mathrm{R}$ & 78 \\
\hline $4-2,6-8$ & 27.87 & $3.34 \mathrm{E}-6$ & -2.9 & 182.0 & -23.7 & 172.1 & $\mathrm{R}$ & 67 \\
\hline $4-2,32-34$ & 28.13 & $3.34 \mathrm{E}-6$ & +14.8 & 16.5 & -8.3 & 4.0 & $\mathrm{~N}$ & 56 \\
\hline $4-2,89-91$ & 28.70 & $2.84 \mathrm{E}-6$ & -26.2 & 209.9 & -24.1 & 216.5 & $\mathrm{R}$ & 429 \\
\hline $4-2,133-135$ & 29.14 & $4.02 \mathrm{E}-6$ & +5.2 & 176.6 & +5.0 & 180.0 & $\mathrm{R}$ & 104 \\
\hline $4-3,19-21$ & 29.50 & $3.73 \mathrm{E}-6$ & +9.3 & 357.2 & +9.4 & 10.7 & $\mathrm{~N}$ & 150 \\
\hline $4-3,29-31$ & 29.60 & $2.48 \mathrm{E}-6$ & +10.9 & 347.3 & +17.7 & 359.4 & $\mathrm{~N}$ & 70 \\
\hline $4-3,39-41$ & 29.70 & $3.96 \mathrm{E}-6$ & +42.1 & 340.2 & +27.2 & 350.3 & $\mathrm{~N}$ & 48 \\
\hline $4-3,49-51$ & 29.80 & $1.17 \mathrm{E}-6$ & -14.6 & 28.4 & +13.1 & 169.2 & $\mathbf{R}$ & 37 \\
\hline $4-3,59-61$ & 29.90 & $1.91 \mathrm{E}-6$ & +46.6 & 345.8 & +6.0 & 177.7 & $\mathbf{R}$ & 32 \\
\hline $4-3,69-71$ & 30.00 & $2.93 \mathrm{E}-6$ & -4.9 & 355.0 & +7.0 & 0.7 & $\mathrm{~N}$ & 57 \\
\hline
\end{tabular}


Appendix A. (Continued).

\begin{tabular}{|c|c|c|c|c|c|c|c|c|}
\hline $\begin{array}{l}\text { Core-Section } \\
\text { (interval in } \mathrm{cm} \text { ) }\end{array}$ & $\begin{array}{l}\text { Sub-bottom } \\
\text { depth } \\
\text { (m) }\end{array}$ & $\underset{(\mathrm{G})}{J_{\mathrm{NRM}}}$ & $I_{\mathrm{NRM}}$ & $D_{\text {NRM }}$ & $I_{\text {stable }}$ & $D_{\text {stable }}$ & Polarity & $\begin{array}{l}\text { MDF } \\
(\mathrm{Oe})\end{array}$ \\
\hline \multicolumn{9}{|l|}{ Hole 575 (Cont.) } \\
\hline $4-3,79-81$ & 30.10 & 1.15 E-6 & +39.6 & 41.7 & +13.5 & 166.1 & $\mathbf{R}$ & 30 \\
\hline $4-3,89-91$ & 30.20 & $1.22 \mathrm{E}-6$ & +37.4 & 343.7 & +18.3 & 145.9 & $\mathbf{R}$ & 33 \\
\hline $4-3,99-101$ & 30.30 & $1.22 \mathrm{E}-6$ & -6.5 & 342.9 & -23.2 & 128.4 & $\mathbf{R}$ & 36 \\
\hline $4-3,109-111$ & 30.40 & 1.34 E-6 & +42.3 & 317.5 & -23.2 & 186.8 & $\mathbf{R}$ & 30 \\
\hline $4-3,119-121$ & 30.50 & 9.44 E-7 & -23.0 & 282.6 & -8.9 & 179.2 & $\mathbf{R}$ & 317 \\
\hline $4-3,129-131$ & 30.60 & $1.21 \mathrm{E}-6$ & +39.0 & 83.3 & -2.4 & 181.7 & $\mathbf{R}$ & 34 \\
\hline $4-3,139-141$ & 30.70 & $1.70 \mathrm{E}-6$ & +29.1 & 57.3 & +24.3 & 39.1 & $\mathrm{~N}$ & 34 \\
\hline $4-4,4-6$ & 30.85 & $3.72 \mathrm{E}-6$ & -4.4 & 353.4 & -8.1 & 5.3 & $\mathrm{~N}$ & 48 \\
\hline $4-4,14-16$ & 30.95 & 3.04 E-6 & +8.7 & 31.9 & -6.0 & 26.6 & $\mathrm{~N}$ & 46 \\
\hline $4-4,24-26$ & 31.05 & $9.17 \mathrm{E}-7$ & +21.3 & 67.0 & +3.8 & 184.7 & $\mathbf{R}$ & 40 \\
\hline $4-4,34-36$ & 31.15 & 8.89 E-7 & +42.8 & 191.0 & +59.1 & 167.1 & $\mathbf{R}$ & 35 \\
\hline $4-4,44-46$ & 31.25 & $5.61 \mathrm{E}-7$ & -15.7 & 1.0 & -14.8 & 160.3 & $\mathbf{R}$ & 35 \\
\hline $4-4,54-56$ & 31.35 & 1.07 E-6 & -5.3 & 40.2 & -0.5 & 35.5 & $\mathbf{N}$ & 37 \\
\hline $4-4,64-66$ & 31.45 & $1.59 \mathrm{E}-6$ & +34.8 & 20.3 & +35.9 & 14.4 & $\mathrm{~N}$ & 35 \\
\hline $4-4,74-76$ & 31.55 & $1.31 \mathrm{E}-6$ & +29.7 & 346.9 & +4.8 & 179.9 & $\mathbf{R}$ & 30 \\
\hline $4-4,84-86$ & 31.65 & $5.42 \mathrm{E}-7$ & -16.5 & 55.7 & -12.2 & 173.0 & $\mathbf{R}$ & 36 \\
\hline $4-4,94-96$ & 31.75 & 5.07 E-7 & +19.8 & 332.9 & -4.1 & 180.4 & $\mathbf{R}$ & 28 \\
\hline $4-4,104-106$ & 31.85 & 6.04 E-7 & -5.8 & 56.6 & +1.8 & 172.2 & $\mathbf{R}$ & 39 \\
\hline $4-4,114-116$ & 31.95 & $6.91 \mathrm{E}-7$ & +40.3 & 340.9 & -16.0 & 166.0 & $\mathbf{R}$ & 29 \\
\hline $4-4,124-126$ & 32.05 & $4.28 \mathrm{E}-7$ & -42.2 & 342.5 & -3.8 & 171.6 & $\mathbf{R}$ & 22 \\
\hline $4-4,134-136$ & 32.15 & $7.12 \mathrm{E}-7$ & +26.7 & 40.1 & +15.4 & 38.8 & $\mathrm{~N}$ & 36 \\
\hline $4-4,144-146$ & 32.25 & 3.85 E-7 & +22.2 & 41.7 & +24.3 & 47.5 & $\mathrm{~N}$ & 41 \\
\hline $4-5,8-10$ & 32.39 & $3.48 \mathrm{E}-7$ & -57.8 & 211.6 & -14.6 & 354.6 & $\mathrm{~N}$ & $>250$ \\
\hline $4-5,64-66$ & 32.95 & $3.70 \mathrm{E}-7$ & +11.2 & 207.4 & +11.2 & 207.4 & $\mathbf{R}$ & 46 \\
\hline $4-5,90-92$ & 33.21 & $1.76 \mathrm{E}-7$ & -39.4 & 177.0 & -35.8 & 180.0 & $\mathrm{~N}$ & 26 \\
\hline $4-5,133-135$ & 33.64 & $1.98 \mathrm{E}-7$ & +32.2 & 2.2 & +15.0 & 0.1 & $\mathrm{~N}$ & $>300$ \\
\hline $4-6,10-12$ & 33.91 & $3.75 \mathrm{E}-7$ & -1.6 & 342.8 & -0.2 & 359.3 & $\mathbf{N}$ & $>300$ \\
\hline $4-6,38-40$ & 34.19 & $4.46 \mathrm{E}-7$ & +20.9 & 188.9 & +7.0 & 3.6 & $\mathrm{~N}$ & 37 \\
\hline $4-6,47-49$ & 34.28 & $4.55 \mathrm{E}-7$ & +61.2 & 27.8 & -15.6 & 183.9 & $\mathbf{R}$ & 30 \\
\hline $4-6,59-61$ & 34.40 & 4.07 E-7 & +25.7 & 77.2 & -33.0 & 158.3 & $\mathbf{R}$ & 42 \\
\hline $4-6,70-72$ & 34.51 & $6.09 \mathrm{E}-7$ & -10.2 & 78.0 & +31.1 & 143.7 & $\mathbf{R}$ & 45 \\
\hline $4-6,82-84$ & 34.63 & $6.95 \mathrm{E}-7$ & -64.0 & 347.4 & -5.9 & 167.0 & $\mathbf{R}$ & 45 \\
\hline $4-6,94-96$ & 34.75 & $4.24 \mathrm{E}-7$ & +56.0 & 325.9 & -14.1 & 181.7 & $\mathbf{R}$ & 389 \\
\hline $4-6,94-96$ & 34.75 & $4.24 \mathrm{E}-7$ & +56.0 & 325.9 & -14.1 & 181.7 & $\mathbf{R}$ & 389 \\
\hline \multicolumn{9}{|l|}{ Hole 575B } \\
\hline $1-1,10-12$ & 3.41 & 3.76 E-6 & +30.7 & 346.0 & +41.9 & 345.0 & $\mathrm{~N}$ & 159 \\
\hline $1-1,20-22$ & 3.51 & 5.23 E-6 & +17.2 & 350.2 & +22.0 & 351.5 & $\mathrm{~N}$ & 97 \\
\hline $1-1,30-32$ & 3.61 & 8.52 E-6 & +13.7 & 358.4 & +14.2 & 357.9 & $\mathrm{~N}$ & 135 \\
\hline $1-1,40-42$ & 3.71 & $3.19 \mathrm{E}-6$ & -10.2 & 354.4 & -15.1 & 357.6 & $\mathrm{~N}$ & 128 \\
\hline $1-1,50-52$ & 3.81 & $1.66 \mathrm{E}-6$ & +6.7 & 3.9 & +3.6 & 355.4 & $\mathrm{~N}$ & 249 \\
\hline $1-1,60-62$ & 3.91 & 1.83 E-6 & +14.0 & 358.4 & +14.3 & 4.9 & $\mathrm{~N}$ & 188 \\
\hline $1-1,70-72$ & 4.01 & 2.11 E-6 & +24.6 & 337.7 & +17.9 & 344.2 & $\mathrm{~N}$ & 284 \\
\hline $1-1,100-102$ & 4.31 & 6.17 E-6 & +15.3 & 1.9 & +18.7 & 4.1 & $\mathrm{~N}$ & 206 \\
\hline $1-1,110-112$ & 4.41 & 7.63 E-6 & +11.0 & 3.5 & +18.1 & 359.9 & $\mathrm{~N}$ & 279 \\
\hline $1-1,120-122$ & 4.51 & 6.19 E-6 & +10.4 & 359.7 & +15.5 & 358.8 & $\mathrm{~N}$ & 126 \\
\hline $1-1,130-132$ & 4.61 & 4.00 E-6 & +11.8 & 357.0 & +16.0 & 357.4 & $\mathrm{~N}$ & 172 \\
\hline $1-1,140-142$ & 4.71 & $5.20 \mathrm{E}-6$ & +8.4 & 2.8 & +13.6 & 3.1 & $\mathrm{~N}$ & 163 \\
\hline $1-2,10-12$ & 4.91 & 6.17 E-6 & +15.3 & 343.0 & +23.8 & 352.0 & $\mathrm{~N}$ & 50 \\
\hline $1-2,20-22$ & 5.01 & 1.67 E-6 & +39.4 & 351.3 & +19.1 & 184.1 & $\mathrm{R}$ & 339 \\
\hline $1-2,30-32$ & 5.11 & 1.77 E-6 & +28.8 & 2.0 & +3.7 & 187.9 & $\mathbf{R}$ & 344 \\
\hline $1-2,40-42$ & 5.21 & $6.93 \mathrm{E}-7$ & -16.2 & 200.0 & -10.3 & 186.2 & $\mathbf{R}$ & 645 \\
\hline $1-2,50-52$ & 5.31 & 1.47 E-6 & +29.4 & 183.6 & +0.4 & 185.4 & $\mathbf{R}$ & 525 \\
\hline $1-2,60-62$ & 5.41 & 1.64 E-6 & +9.7 & 6.6 & +5.4 & 187.7 & $\mathbf{R}$ & 349 \\
\hline $1-2,70-72$ & 5.51 & 3.74 E-6 & +5.6 & 13.7 & +32.4 & 359.5 & $\mathrm{~N}$ & 43 \\
\hline $1-2,100-102$ & 5.81 & 1.42 E-6 & -47.5 & 46.9 & +22.6 & 207.7 & $\mathbf{R}$ & 31 \\
\hline $1-2,112-114$ & 5.93 & 1.55 E-6 & -69.3 & 20.6 & -21.3 & 188.7 & $\mathbf{R}$ & 379 \\
\hline $1-2,122-124$ & 6.03 & 1.45 E-6 & +55.7 & 317.0 & +60.6 & 157.7 & $\mathbf{R}$ & 187 \\
\hline $1-2,133-135$ & 6.14 & $1.38 \mathrm{E}-6$ & -67.6 & 277.0 & -39.4 & 191.5 & $\vec{R}$ & 349 \\
\hline $1-2,144-146$ & 6.25 & $6.05 \mathrm{E}-7$ & -29.7 & 183.0 & -4.7 & 176.8 & $\mathbf{R}$ & 497 \\
\hline $1-3,2-4$ & 6.33 & $6.91 \mathrm{E}-7$ & +15.8 & 231.5 & +3.4 & 170.5 & $\mathbf{R}$ & 317 \\
\hline $1-3,10-12$ & 6.41 & 1.16 E-6 & -44.0 & 259.2 & -19.0 & 187.0 & $\mathbf{R}$ & 266 \\
\hline $1-4,13-15$ & 7.94 & 1.96 E-6 & -49.9 & 287.9 & -55.3 & 340.3 & $\mathbf{N}$ & 83 \\
\hline $1-5,12-14$ & 9.43 & $6.84 \mathrm{E}-7$ & -26.0 & 252.3 & \multicolumn{2}{|c|}{ no stable } & & $>500$ \\
\hline $1-5,97-99$ & 10.28 & 5.35 E-6 & -7.0 & 2.5 & \multicolumn{2}{|c|}{ no stable } & & 40 \\
\hline $1-6,69-71$ & 11.50 & 1.79 E-6 & -38.7 & 276.7 & \multicolumn{2}{|c|}{ no stable } & & 254 \\
\hline $1-6,141-143$ & 12.22 & 2.71 E-6 & -10.1 & 306.5 & \multicolumn{2}{|c|}{ no stable } & & 75 \\
\hline $2-1,80-82$ & 12.81 & 3.01 E-6 & +8.1 & 43.6 & +40.5 & 32.4 & $\mathrm{~N}$ & 38 \\
\hline $2-1,90-92$ & 12.91 & 4.43 E-6 & +6.3 & 30.6 & +9.6 & 6.3 & $\mathrm{~N}$ & 50 \\
\hline $2-1,100-102$ & 13.01 & 4.50 E-6 & +12.3 & 32.3 & +13.0 & 15.4 & $\mathbf{N}$ & 43 \\
\hline $2-1,110-112$ & 13.11 & $2.93 \mathrm{E}-6$ & +7.4 & 39.1 & -9.9 & 178.1 & $\mathbf{R}$ & 29 \\
\hline $2-1,120-122$ & 13.21 & $3.29 \mathrm{E}-6$ & +12.4 & 35.9 & -18.6 & 181.8 & $\mathbf{R}$ & 28 \\
\hline $2-1,130-132$ & 13.31 & $2.12 \mathrm{E}-6$ & +4.4 & 54.8 & -17.0 & 186.9 & $\mathbf{R}$ & 48 \\
\hline
\end{tabular}


Appendix A. (Continued).

\begin{tabular}{|c|c|c|c|c|c|c|c|c|}
\hline $\begin{array}{c}\text { Core-Section } \\
\text { (interval in cm) }\end{array}$ & $\begin{array}{l}\text { Sub-bottom } \\
\text { depth } \\
\text { (m) }\end{array}$ & $\underset{(\mathrm{G})}{J_{\mathrm{NRM}}}$ & $I_{\mathrm{NRM}}$ & $D_{\mathrm{NRM}}$ & $I_{\text {stable }}$ & $D_{\text {stable }}$ & Polarity & $\begin{array}{l}\text { MDF } \\
(\mathrm{Oe})\end{array}$ \\
\hline \multicolumn{9}{|l|}{ Hole 575B (Cont.) } \\
\hline $2-1,140-142$ & 13.41 & $2.40 \mathrm{E}-6$ & -9.7 & 47.3 & -29.2 & 186.1 & $\mathrm{R}$ & 43 \\
\hline $2-2,2-4$ & 13.53 & $3.65 \mathrm{E}-6$ & +20.5 & 71.0 & -23.8 & 173.0 & $\mathrm{R}$ & 43 \\
\hline $2-2,12-14$ & 13.63 & $3.09 \mathrm{E}-6$ & +28.8 & 55.4 & -0.2 & 166.6 & $\mathrm{R}$ & 46 \\
\hline $2-2,22-24$ & 13.73 & $2.28 \mathrm{E}-6$ & +27.5 & 71.5 & -4.3 & 170.1 & $\mathbf{R}$ & 325 \\
\hline $2-2,32-34$ & 13.83 & $1.98 \mathrm{E}-6$ & +23.0 & 84.2 & -3.9 & 166.1 & $\mathrm{R}$ & 355 \\
\hline $2-2,42-44$ & 13.93 & $1.95 \mathrm{E}-6$ & +24.4 & 77.5 & -0.4 & 173.5 & $\mathbf{R}$ & 339 \\
\hline $2-2,52-54$ & 14.03 & $2.41 \mathrm{E}-6$ & +41.0 & 86.5 & +12.2 & 172.7 & $\mathbf{R}$ & 341 \\
\hline $2-2,62-64$ & 14.13 & $2.41 \mathrm{E}-6$ & +17.3 & 113.8 & -3.7 & 170.1 & $\mathbf{R}$ & 365 \\
\hline $2-2,72-74$ & 14.23 & $2.21 \mathrm{E}-6$ & +27.5 & 98.8 & +2.4 & 174.0 & $\mathrm{R}$ & 352 \\
\hline $2-2,82-84$ & 14.33 & $2.44 \mathrm{E}-6$ & +8.2 & 111.7 & -3.5 & 167.3 & $\mathbf{R}$ & 342 \\
\hline $2-2,92-94$ & 14.43 & $2.01 \mathrm{E}-6$ & +2.1 & 110.4 & -9.5 & 173.2 & $\mathbf{R}$ & 345 \\
\hline $2-2,102-104$ & 14.53 & $1.54 \mathrm{E}-6$ & -1.0 & 65.7 & -3.0 & 168.1 & $\mathbf{R}$ & 381 \\
\hline $2-2,112-114$ & 14.63 & $3.05 \mathrm{E}-6$ & +7.8 & 23.6 & +8.3 & 168.2 & $R$ & 33 \\
\hline $2-2,122-124$ & 14.73 & $3.61 \mathrm{E}-6$ & -2.5 & 15.9 & +11.5 & 347.4 & $\mathrm{~N}$ & 43 \\
\hline $2-2,132-134$ & 14.83 & $4.49 \mathrm{E}-6$ & -1.8 & 20.8 & +4.4 & 358.3 & $\mathrm{~N}$ & 64 \\
\hline $2-2,142-144$ & 14.93 & $3.88 \mathrm{E}-6$ & +4.6 & 15.2 & +19.3 & 355.6 & $\mathrm{~N}$ & 56 \\
\hline $2-3,2-4$ & 15.03 & $5.17 \mathrm{E}-6$ & +30.4 & 23.7 & +64.3 & 347.8 & $\mathrm{~N}$ & 36 \\
\hline $2-3,12-14$ & 15.13 & $5.03 \mathrm{E}-6$ & +22.9 & 21.5 & +27.2 & 358.7 & $\mathrm{~N}$ & 44 \\
\hline $2-3,22-24$ & 15.23 & $4.87 \mathrm{E}-6$ & +17.6 & 18.1 & +12.0 & 357.6 & $\mathrm{~N}$ & 48 \\
\hline $2-3,32-34$ & 15.33 & $5.29 \mathrm{E}-6$ & +15.6 & 15.3 & +10.5 & 358.9 & $\mathrm{~N}$ & 53 \\
\hline $2-3,42-44$ & 15.43 & $5.02 \mathrm{E}-6$ & +15.4 & 22.3 & +17.5 & 2.7 & $\mathrm{~N}$ & 63 \\
\hline $2-3,52-54$ & 15.53 & $5.68 \mathrm{E}-6$ & +17.3 & 13.1 & +13.3 & 355.0 & $\mathrm{~N}$ & 79 \\
\hline $2-3,62-64$ & 15.63 & $4.37 \mathrm{E}-6$ & +13.4 & 18.6 & +21.1 & 356.2 & $\mathrm{~N}$ & 67 \\
\hline $2-3,72-74$ & 15.73 & $2.90 \mathrm{E}-6$ & +10.8 & 19.0 & +14.4 & 350.2 & $\mathrm{~N}$ & 55 \\
\hline $2-3,82-84$ & 15.83 & $1.67 \mathrm{E}-6$ & +6.3 & 35.4 & -5.6 & 184.8 & $\mathrm{R}$ & 41 \\
\hline $2-3,92-94$ & 15.93 & $1.42 \mathrm{E}-6$ & +13.3 & 47.0 & +3.2 & 183.6 & $\mathrm{R}$ & 41 \\
\hline $2-3,102-104$ & 16.03 & $1.29 \mathrm{E}-6$ & -0.9 & 81.8 & -5.0 & 178.6 & $\mathrm{R}$ & 337 \\
\hline $2-3,112-114$ & 16.13 & $1.63 \mathrm{E}-6$ & -7.4 & 118.4 & -10.8 & 176.4 & $\mathbf{R}$ & 329 \\
\hline $2-3,122-124$ & 16.23 & $1.29 \mathrm{E}-6$ & -14.4 & 97.4 & -5.9 & 174.9 & $\mathrm{R}$ & 314 \\
\hline $2-3,132-134$ & 16.33 & $1.18 \mathrm{E}-6$ & +5.1 & 65.7 & +11.7 & 176.6 & $\mathrm{R}$ & 40 \\
\hline $2-3,142-144$ & 16.43 & $1.42 \mathrm{E}-6$ & -3.3 & 46.5 & +7.1 & 174.0 & $\mathrm{R}$ & 41 \\
\hline $2-4,2-4$ & 16.53 & $4.47 \mathrm{E}-6$ & +26.7 & 35.6 & +16.1 & 180.3 & $\mathrm{R}$ & 32 \\
\hline $2-4,12-14$ & 16.63 & $5.35 \mathrm{E}-6$ & +16.5 & 24.5 & +28.2 & 10.1 & $\mathrm{~N}$ & 43 \\
\hline $2-4,22-24$ & 16.73 & $4.77 \mathrm{E}-6$ & +12.5 & 24.2 & +16.4 & 1.1 & $\mathrm{~N}$ & 50 \\
\hline $2-4,32-34$ & 16.83 & 5.57 E-6 & 16.6 & 21.4 & +15.2 & 359.4 & $\mathrm{~N}$ & 58 \\
\hline $2-4,42-44$ & 16.93 & $5.93 \mathrm{E}-6$ & +12.9 & 18.7 & +8.2 & 357.4 & $\mathrm{~N}$ & 74 \\
\hline $2-4,52-54$ & 17.03 & $6.63 \mathrm{E}-6$ & +13.3 & 19.7 & +14.2 & 6.0 & $\mathrm{~N}$ & 84 \\
\hline $2-4,72-74$ & 17.23 & 3.07 E-6 & +0.5 & 47.8 & -39.3 & 156.4 & $N$ & 37 \\
\hline $2-4,82-84$ & 17.33 & 3.74 E-6 & +18.3 & 20.2 & +28.2 & 348.1 & $\mathrm{~N}$ & 54 \\
\hline $2-4,92-94$ & 17.43 & 4.77 E-6 & +20.7 & 31.9 & +26.1 & 15.0 & $\mathrm{~N}$ & 81 \\
\hline $2-4,102-104$ & 17.53 & $4.75 \mathrm{E}-6$ & -45.5 & 11.4 & -67.6 & 304.4 & $\mathrm{~N}$ & 374 \\
\hline $2-4,122-114$ & 17.63 & $4.08 \mathrm{E}-6$ & +11.7 & 19.8 & +16.1 & 7.5 & $\mathrm{~N}$ & 93 \\
\hline $2-4,122-124$ & 17.73 & $3.99 \mathrm{E}-6$ & +10.7 & 25.9 & +12.5 & 2.2 & $\mathrm{~N}$ & 97 \\
\hline $2-4,132-134$ & 17.83 & $2.81 \mathrm{E}-6$ & +11.6 & 20.3 & +19.1 & 352.7 & $\mathrm{~N}$ & 99 \\
\hline $2-4,142-144$ & 17.93 & 3.77 E-6 & +11.3 & 25.9 & +18.6 & 359.7 & $\mathrm{~N}$ & 127 \\
\hline $2-5,2-4$ & 18.03 & $3.59 \mathrm{E}-6$ & +33.9 & 41.6 & +33.5 & 359.7 & $\mathrm{~N}$ & 45 \\
\hline $2-5,12-14$ & 18.13 & $3.59 \mathrm{E}-6$ & +32.8 & 54.9 & +54.5 & 30.7 & $\mathrm{~N}$ & 40 \\
\hline $2-5,22-24$ & 18.23 & 2.97 E-6 & +36.7 & 74.0 & +13.8 & 181.3 & $\mathrm{R}$ & 34 \\
\hline $2-5,32-34$ & 18.33 & 2.32 E-6 & +22.7 & 47.1 & +19.0 & 184.8 & $\mathrm{R}$ & 34 \\
\hline $2-5,42-44$ & 18.43 & $2.20 \mathrm{E}-6$ & +18.6 & 41.9 & +13.7 & 180.7 & $\mathbf{R}$ & 31 \\
\hline $2-5,52-54$ & 18.53 & $3.75 \mathrm{E}-6$ & +24.8 & 51.6 & +4.2 & 180.6 & $\mathbf{R}$ & 31 \\
\hline $2-5,62-64$ & 18.63 & $3.02 \mathrm{E}-6$ & +28.1 & 52.2 & +15.8 & 191.7 & $\mathbf{R}$ & 34 \\
\hline $2-5,72-74$ & 18.73 & $2.39 \mathrm{E}-6$ & +34.0 & 62.5 & +19.9 & 186.5 & $\mathbf{R}$ & 38 \\
\hline $2-5,82-84$ & 18.83 & 2.03 E-6 & +27.5 & 42.0 & +47.5 & 196.3 & $\mathbf{R}$ & 43 \\
\hline $2-5,92-94$ & 18.93 & $1.52 \mathrm{E}-6$ & +25.3 & 51.7 & +20.4 & 188.4 & $\mathrm{R}$ & 39 \\
\hline $2-5,102-104$ & 19.03 & $1.09 \mathrm{E}-6$ & +20.9 & 94.0 & -7.8 & 186.6 & R & 364 \\
\hline $2-5,112-114$ & 19.13 & $1.54 \mathrm{E}-6$ & +31.9 & 76.4 & +14.6 & 194.5 & R & 270 \\
\hline $2-5,122-124$ & 19.23 & $1.58 \mathrm{E}-6$ & +38.2 & 64.0 & +37.5 & 192.0 & $R$ & 88 \\
\hline $2-5,132-134$ & 19.33 & $1.06 \mathrm{E}-6$ & +16.2 & 69.5 & +18.9 & 180.1 & R & 49 \\
\hline $2-5,142-144$ & 19.43 & $9.28 \mathrm{E}-7$ & +29.1 & 87.0 & +23.3 & 195.4 & R & 316 \\
\hline $2-6,2-4$ & 19.53 & $1.17 \mathrm{E}-6$ & +38.9 & 79.8 & +12.2 & 188.5 & R & 46 \\
\hline $2-6,12-14$ & 19.63 & 1.64 E-6 & +26.8 & 60.6 & +17.3 & 189.6 & $\mathbf{R}$ & 40 \\
\hline $2-6,22-24$ & 19.73 & $1.43 \mathrm{E}-6$ & +22.8 & 73.6 & -14.0 & 200.9 & R & 31 \\
\hline $2-6,32-34$ & 19.83 & $1.28 \mathrm{E}-6$ & +24.4 & 75.2 & -8.7 & 199.2 & $\mathrm{R}$ & 36 \\
\hline $2-6,42-44$ & 19.93 & 8.76 E-7 & +30.4 & 98.0 & -0.5 & 182.7 & $\mathbf{R}$ & 300 \\
\hline $2-6,52-54$ & 20.03 & $8.29 \mathrm{E}-7$ & +19.4 & 112.6 & +5.5 & 185.0 & $\mathrm{R}$ & 44 \\
\hline $2-5,62-64$ & 20.13 & $1.15 \mathrm{E}-6$ & +24.6 & 120.1 & +17.6 & 175.3 & R & 74 \\
\hline $2-6,72-74$ & 20.23 & $1.07 \mathrm{E}-6$ & +11.5 & 59.0 & +25.5 & 165.7 & $\mathrm{R}$ & 44 \\
\hline $2-6,82-84$ & 20.33 & 7.47 E-7 & +16.6 & 68.4 & +0.5 & 161.0 & $\mathrm{R}$ & 349 \\
\hline $2-6,92-94$ & 20.43 & 1.17 E-6 & -3.1 & 70.7 & -23.5 & 132.6 & $\mathrm{R}$ & 38 \\
\hline $2-6,102-104$ & 20.53 & $6.39 \mathrm{E}-7$ & +4.2 & 45.0 & +4.2 & 45.0 & $\mathbf{R}$ & 333 \\
\hline $2-6,112-114$ & 20.63 & 7.04 E-7 & +15.3 & 56.7 & +63.7 & 185.4 & $\mathbf{R}$ & 48 \\
\hline $2-6,122-124$ & 20.73 & $6.29 \mathrm{E}-7$ & +16.1 & 78.3 & +6.8 & 180.4 & $\mathbf{R}$ & 37 \\
\hline $2-6,132-134$ & 20.83 & $4.46 \mathrm{E}-7$ & +11.9 & 89.4 & +29.0 & 164.8 & $\mathrm{R}$ & 118 \\
\hline $2-6,142-144$ & 20.93 & $3.29 \mathrm{E}-7$ & +14.4 & 65.0 & +83.0 & 210.6 & $\mathrm{R}$ & 90 \\
\hline
\end{tabular}


Appendix A. (Continued).

\begin{tabular}{|c|c|c|c|c|c|c|c|c|}
\hline $\begin{array}{l}\text { Core-Section } \\
\text { (interval in } \mathrm{cm} \text { ) }\end{array}$ & $\begin{array}{l}\text { Sub-bottom } \\
\text { depth } \\
\text { (m) }\end{array}$ & $\underset{(\mathrm{G})}{J_{\mathrm{NRM}}}$ & $I_{\mathrm{NRM}}$ & $D_{\mathrm{NRM}}$ & $I_{\text {stable }}$ & $D_{\text {stable }}$ & Polarity & $\begin{array}{l}\text { MDF } \\
\text { (Oe) }\end{array}$ \\
\hline \multicolumn{9}{|l|}{ Hole 575B (Cont.) } \\
\hline $2-7,2-4$ & 21.03 & 3.48 E-7 & +29.0 & 67.8 & \multicolumn{2}{|c|}{ no stable } & & \\
\hline $2-7,12-14$ & 21.13 & 4.43 E-7 & +45.3 & 9.5 & +43.6 & 10.0 & $\mathrm{~N}$ & 63 \\
\hline $2-7,22-24$ & 21.23 & 2.95 E-7 & +45.5 & 9.4 & +51.3 & 12.9 & $\mathrm{~N}$ & 99 \\
\hline $2-7,32-34$ & 21.33 & 2.57 E-7 & +64.3 & 15.4 & +62.7 & 14.2 & $\mathrm{~N}$ & 134 \\
\hline $2-7,42-44$ & 21.43 & $4.44 \mathrm{E}-7$ & +56.2 & 327.3 & +60.3 & 320.8 & $\mathrm{~N}$ & 213 \\
\hline $3-1,58-60$ & 21.59 & 1.70 E-6 & +47.1 & 43.4 & +48.9 & 353.2 & & 37 \\
\hline $3-1,68-70$ & 21.69 & $5.34 \mathrm{E}-7$ & +38.7 & 356.9 & +31.2 & 352.4 & & 220 \\
\hline $3-1,78-80$ & 21.79 & 7.86 E-6 & +12.3 & 46.4 & -49.1 & 96.4 & & 41 \\
\hline $3-1,88-90$ & 21.89 & 7.37 E-7 & +1.4 & 39.1 & -30.4 & 35.3 & & 65 \\
\hline $3-1,98-100$ & 21.99 & $7.82 \mathrm{E}-7$ & +7.2 & 45.8 & +8.3 & 40.4 & & 97 \\
\hline $3-1,108-110$ & 22.09 & $9.46 \mathrm{E}-7$ & +12.2 & 51.8 & -15.0 & 48.2 & & 75 \\
\hline $3-1,118-120$ & 22.19 & $9.97 \mathrm{E}-7$ & -6.2 & 25.7 & -11.9 & 23.2 & & 109 \\
\hline $3-1,128-130$ & 22.29 & 2.97 E-6 & -3.0 & 14.5 & -23.6 & 12.6 & & 61 \\
\hline $3-1,138-140$ & 22.39 & $2.21 \mathrm{E}-6$ & -14.7 & 23.0 & -48.6 & 15.5 & & 97 \\
\hline $3-2,8-10$ & 22.59 & 1.98 E-6 & -3.2 & 23.3 & -80.6 & 353.6 & & 37 \\
\hline $3-2,30-32$ & 22.81 & 2.00 E-6 & +4.4 & 29.7 & -67.4 & 192.0 & & 34 \\
\hline $3-2,50-52$ & 23.01 & 2.19 E-6 & +7.5 & 30.3 & -77.9 & 150.1 & & 33 \\
\hline $3-2,136-138$ & 23.87 & 2.22 E-6 & -29.0 & 23.4 & -61.6 & 18.0 & & 98 \\
\hline $3-3,12-14$ & 24.13 & 2.94 E-6 & -23.7 & 15.7 & -70.0 & 13.7 & & 74 \\
\hline $3-3,106-108$ & 25.07 & 1.44 E-6 & -8.5 & 4.2 & -38.8 & 9.3 & & 220 \\
\hline $3-4,11-13$ & 25.62 & $1.33 \mathrm{E}-6$ & -12.1 & 11.9 & -26.4 & 3.8 & & 52 \\
\hline $3-4,39-41$ & 25.90 & 1.32 E-6 & -18.2 & 15.3 & -51.0 & 12.4 & & 47 \\
\hline $3-4,59-61$ & 26.10 & 1.84 E-6 & +16.5 & 18.0 & +33.5 & 359.7 & & 139 \\
\hline $3-4,100-102$ & 26.51 & 2.06 E-6 & -3.6 & 1.8 & -15.6 & 14.5 & & 96 \\
\hline $3-4,110-112$ & 26.61 & 1.96 E-6 & -0.5 & 1.6 & +10.3 & 346.2 & & 93 \\
\hline $3-4,120-122$ & 26.71 & 3.04 E-6 & +17.4 & 352.9 & +21.6 & 330.9 & & 99 \\
\hline $3-4,130-132$ & 26.81 & 2.36 E-6 & +18.2 & 346.2 & +20.6 & 334.3 & & 89 \\
\hline $3-4,140-142$ & 26.91 & 2.68 E-6 & +17.1 & 347.3 & +20.7 & 335.0 & & 91 \\
\hline $3-5,8-10$ & 27.09 & 2.86 E-6 & +41.7 & 16.3 & +29.7 & 356.2 & & 42 \\
\hline $3-5,18-20$ & 27.19 & $2.58 \mathrm{E}-6$ & +41.7 & 5.8 & +25.5 & 339.3 & & 51 \\
\hline $3-5,28-30$ & 27.29 & 2.51 E-6 & +45.0 & 25.6 & +31.2 & 340.4 & & 48 \\
\hline $3-5,38-40$ & 27.39 & 3.56 E-6 & +16.9 & 352.9 & +19.5 & 335.7 & & 64 \\
\hline $3-5,48-50$ & 27.49 & $3.02 \mathrm{E}-6$ & +11.2 & 349.6 & +10.3 & 332.7 & & 98 \\
\hline $3-5,58-60$ & 27.59 & 2.94 E-6 & +13.5 & 346.5 & +15.7 & 334.2 & & 107 \\
\hline $3-5,68-70$ & 27.69 & 1.66 E-6 & +21.2 & 359.1 & +32.7 & 351.0 & & 71 \\
\hline $3-5,78-80$ & 27.79 & $1.72 \mathrm{E}-6$ & +19.5 & 349.7 & +27.9 & 332.2 & & 96 \\
\hline $3-5,88-90$ & 27.89 & $1.76 \mathrm{E}-6$ & +12.3 & 349.5 & +16.7 & 337.1 & & 205 \\
\hline $3-5,98-100$ & 27.99 & $1.20 \mathrm{E}-6$ & +23.9 & 353.5 & +26.9 & 350.3 & & 177 \\
\hline $3-5,108-110$ & 28.09 & $7.34 \mathrm{E}-7$ & +16.4 & 7.1 & +25.4 & 356.5 & & 228 \\
\hline $3-5,118-120$ & 28.19 & $7.57 \mathrm{E}-7$ & +8.8 & 356.8 & +26.7 & 348.3 & & 208 \\
\hline $3-5,128-130$ & 28.29 & $9.83 \mathrm{E}-7$ & +12.5 & 355.0 & +26.6 & 340.4 & & 246 \\
\hline $3-5,138-140$ & 28.39 & $7.31 \mathrm{E}-7$ & +22.1 & 359.4 & +26.3 & 348.3 & & 235 \\
\hline $3-5,146-148$ & 28.47 & 1.71 E-6 & +12.4 & 292.8 & +9.3 & 316.0 & & 87 \\
\hline $3-6,8-10$ & 28.59 & $2.50 \mathrm{E}-6$ & +25.4 & 6.2 & +18.6 & 344.2 & & 75 \\
\hline $3-6,18-20$ & 28.69 & 1.47 E-6 & +17.7 & 6.4 & +26.5 & 345.4 & & 98 \\
\hline $3-6,28-30$ & 28.79 & $1.79 \mathrm{E}-6$ & +14.1 & 353.8 & +16.7 & 335.1 & & 178 \\
\hline $3-6,38-40$ & 28.89 & 7.94 E-7 & +24.7 & 7.6 & +31.8 & 346.4 & & 163 \\
\hline $3-6,48-50$ & 28.99 & $8.12 \mathrm{E}-7$ & +30.0 & 34.6 & +35.8 & 28.5 & & 99 \\
\hline $3-6,58-60$ & 29.09 & $7.92 \mathrm{E}-7$ & +27.6 & 53.7 & +42.7 & 64.9 & & 137 \\
\hline $3-6,68-70$ & 29.19 & $6.77 \mathrm{E}-7$ & +30.2 & 42.7 & +40.7 & 15.1 & & 188 \\
\hline $3-6,82-84$ & 29.33 & $7.48 \mathrm{E}-7$ & +48.5 & 51.5 & +39.0 & 35.5 & & 213 \\
\hline $3-6,95-97$ & 29.46 & $6.08 \mathrm{E}-7$ & +7.1 & 7.7 & +20.4 & 8.5 & & 145 \\
\hline $3-6,105-107$ & 29.56 & $6.55 \mathrm{E}-7$ & +2.3 & 18.1 & +27.4 & 18.2 & & 173 \\
\hline $4-1,26-28$ & 29.77 & $10.0 \mathrm{E}-7$ & +22.8 & 21.4 & +44.2 & 337.7 & & 149 \\
\hline $4-1,41-43$ & 29.92 & 9.75 E-7 & +22.0 & 13.8 & +62.8 & 320.6 & & 120 \\
\hline $4-1,56-58$ & 30.07 & $9.25 \mathrm{E}-7$ & +27.2 & 5.8 & +59.0 & 357.9 & & 173 \\
\hline $4-1,74-76$ & 30.25 & $9.52 \mathrm{E}-7$ & +25.3 & 352.1 & +52.5 & 345.3 & & 223 \\
\hline $4-1,92-94$ & 30.43 & $6.79 \mathrm{E}-7$ & +32.4 & 0.2 & +50.8 & 349.4 & & 255 \\
\hline $4-1,114-116$ & 30.65 & $7.22 \mathrm{E}-7$ & +24.1 & 5.2 & +40.7 & 351.3 & & 309 \\
\hline $4-1,136-138$ & 30.87 & $6.48 \mathrm{E}-7$ & +33.7 & 359.3 & +42.8 & 355.6 & & 313 \\
\hline $4-2,11-13$ & 31.12 & $6.62 \mathrm{E}-7$ & +31.6 & 2.7 & +45.0 & 0.7 & & 207 \\
\hline $4-2,26-28$ & 31.27 & $8.57 \mathrm{E}-7$ & +31.7 & 10.1 & +44.9 & 3.9 & & 286 \\
\hline $4-2,41-43$ & 31.42 & 8.55 E-7 & +35.2 & 3.5 & +57.2 & 348.2 & & 291 \\
\hline $4-2,56-58$ & 31.57 & 1.10 E-6 & +46.7 & 5.4 & +46.0 & 9.1 & & 266 \\
\hline $4-2,71-73$ & 31.72 & 7.94 E-7 & +36.3 & 1.1 & +39.7 & 359.3 & & 298 \\
\hline $4-2,86-88$ & 31.87 & $1.00 \mathrm{E}-6$ & +41.0 & 11.5 & +34.9 & 2.9 & & 316 \\
\hline $4-2,101-103$ & 32.02 & 5.92 E-7 & +46.6 & 358.3 & +45.9 & 355.9 & & 355 \\
\hline $4-2,116-118$ & 32.17 & 8.46 E-7 & +69.3 & 37.0 & +61.8 & 359.7 & & 305 \\
\hline $4-2,131-133$ & 32.32 & $7.28 \mathrm{E}-7$ & +83.7 & 81.7 & +69.0 & 334.7 & & 328 \\
\hline $4-2,146-148$ & 32.47 & 1.01 E-6 & +57.3 & 46.0 & +53.7 & 355.9 & & 306 \\
\hline $4-3,11-13$ & 32.62 & 1.96 E-6 & +52.2 & 24.3 & +65.0 & 28.5 & & 50 \\
\hline $4-3,26-28$ & 32.77 & $1.61 \mathrm{E}-6$ & +46.7 & 10.3 & +54.7 & 5.1 & & 97 \\
\hline $4-3,41-43$ & 32.92 & $1.52 \mathrm{E}-6$ & +35.1 & 11.7 & +38.7 & 4.8 & & 163 \\
\hline
\end{tabular}


Appendix A. (Continued).

\begin{tabular}{|c|c|c|c|c|c|c|c|c|}
\hline $\begin{array}{l}\text { Core-Section } \\
\text { (interval in cm) }\end{array}$ & $\begin{array}{l}\text { Sub-bottom } \\
\text { depth } \\
\text { (m) }\end{array}$ & $\begin{array}{c}J_{\mathrm{NRM}} \\
(\mathrm{G})\end{array}$ & $I_{\mathrm{NRM}}$ & $D_{\mathrm{NRM}}$ & $I_{\text {stable }}$ & $D_{\text {stable }}$ & Polarity & $\begin{array}{l}\text { MDF } \\
\text { (Oe) }\end{array}$ \\
\hline \multicolumn{9}{|l|}{ Hole 575B (Cont.) } \\
\hline $4-3,56-58$ & 33.07 & 9.81 E-7 & +37.9 & 16.2 & +48.1 & 2.9 & & 236 \\
\hline $4-3,71-73$ & 33.22 & $9.30 \mathrm{E}-7$ & +25.4 & 5.8 & +35.3 & 356.1 & & 195 \\
\hline $4-3,91-93$ & 33.42 & $1.93 \mathrm{E}-6$ & +44.2 & 13.7 & +46.9 & 1.0 & & 239 \\
\hline $4-3,114-116$ & 33.65 & $1.29 \mathrm{E}-6$ & +34.1 & 12.2 & +43.0 & 4.0 & & 208 \\
\hline $4-3,136-138$ & 33.87 & $2.10 \mathrm{E}-6$ & +37.1 & 16.5 & +39.8 & 356.8 & & 235 \\
\hline $4-4,11-13$ & 34.12 & $4.29 \mathrm{E}-6$ & +42.9 & 12.1 & +52.8 & 30.6 & & 45 \\
\hline $4-4,26-28$ & 34.27 & $3.32 \mathrm{E}-6$ & +55.9 & 3.5 & +67.4 & 9.8 & & 100 \\
\hline $4-4,41-43$ & 34.42 & $3.05 \mathrm{E}-6$ & +52.2 & 10.8 & +60.1 & 15.2 & & 171 \\
\hline $4-4,56-58$ & 34.57 & $2.77 \mathrm{E}-6$ & +49.9 & 19.9 & +50.3 & 24.8 & & 203 \\
\hline $4-4,71-73$ & 34.72 & 2.04 E-6 & +54.7 & 331.5 & +67.8 & 354.9 & & 215 \\
\hline $4-4,86-88$ & 34.87 & $1.97 \mathrm{E}-6$ & +68.1 & 8.6 & +73.5 & 340.7 & & 198 \\
\hline $4-4,101-103$ & 35.02 & $1.39 \mathrm{E}-6$ & +81.3 & 242.3 & +67.2 & 223.0 & & 260 \\
\hline $4-4,116-118$ & 35.17 & $1.75 \mathrm{E}-6$ & +82.8 & 279.1 & +71.7 & 274.9 & & 276 \\
\hline $4-4,131-133$ & 35.32 & $2.04 \mathrm{E}-6$ & +82.0 & 32.9 & +76.2 & 311.4 & & 265 \\
\hline $4-4,146-148$ & 35.47 & 2.37 E-6 & +81.2 & 242.3 & +66.5 & 257.9 & & 261 \\
\hline $4-5,11-13$ & 35.62 & $1.84 \mathrm{E}-6$ & +81.2 & 225.0 & +69.4 & 244.5 & & 222 \\
\hline $4-5,26-28$ & 35.77 & $2.24 \mathrm{E}-6$ & +53.0 & 175.7 & +50.3 & 214.8 & & 210 \\
\hline $4-5,41-43$ & 35.92 & $2.62 \mathrm{E}-6$ & +62.9 & 163.4 & +68.4 & 230.4 & & 180 \\
\hline $4-5,56-58$ & 36.07 & $9.97 \mathrm{E}-7$ & +84.0 & 66.1 & +75.3 & 321.4 & & 277 \\
\hline $4-5,71-73$ & 36.22 & $8.08 \mathrm{E}-7$ & +68.2 & 45.8 & +74.0 & 348.9 & & 306 \\
\hline $4-5,86-88$ & 36.37 & $1.35 \mathrm{E}-6$ & +50.8 & 32.6 & +51.7 & 16.1 & & 269 \\
\hline $4-5,101-103$ & 36.52 & $6.71 \mathrm{E}-7$ & +83.3 & 35.0 & +69.0 & 285.5 & & 264 \\
\hline $4-5,116-118$ & 36.67 & $1.13 \mathrm{E}-6$ & +40.3 & 195.0 & +48.4 & 228.0 & & 292 \\
\hline $4-5,131-133$ & 36.82 & $6.27 \mathrm{E}-7$ & +49.8 & 130.2 & +69.9 & 323.5 & & 358 \\
\hline $4-5,146-148$ & 36.97 & $7.31 \mathrm{E}-7$ & +43.3 & 107.5 & +74.4 & 335.6 & & 280 \\
\hline $4-6,11-13$ & 37.12 & $3.07 \mathrm{E}-6$ & +52.4 & 8.9 & +59.4 & 7.3 & & 40 \\
\hline $4-6,26-28$ & 37.27 & $1.13 \mathrm{E}-6$ & +84.2 & 231.2 & -11.2 & 194.3 & & 388 \\
\hline $4-6,41-43$ & 37.42 & $2.05 \mathrm{E}-6$ & +64.6 & 359.9 & +68.7 & 262.6 & & 56 \\
\hline $4-6,56-58$ & 37.57 & $1.88 \mathrm{E}-6$ & +76.0 & 336.7 & +52.0 & 239.4 & & 85 \\
\hline $4-6,71-73$ & 37.72 & $1.38 \mathrm{E}-6$ & +79.6 & 327.4 & +52.3 & 243.0 & & 121 \\
\hline $4-6,86-88$ & 37.87 & $1.92 \mathrm{E}-6$ & +76.9 & 173.9 & +46.6 & 213.6 & & 130 \\
\hline $4-6,101-103$ & 38.02 & $1.20 \mathrm{E}-6$ & +72.2 & 186.2 & +50.5 & 235.3 & & 142 \\
\hline $4-6,116-118$ & 38.17 & $1.21 \mathrm{E}-6$ & +57.2 & 202.1 & +25.2 & 221.9 & & 235 \\
\hline $4-6,131-133$ & 38.32 & $1.22 \mathrm{E}-6$ & +54.5 & 190.8 & +22.8 & 217.8 & & 219 \\
\hline $4-6,146-148$ & 38.47 & $1.22 \mathrm{E}-6$ & +51.1 & 179.8 & +22.6 & 225.1 & & 191 \\
\hline $4-7,11-13$ & 38.62 & $9.08 \mathrm{E}-7$ & +51.2 & 200.5 & +30.4 & 221.7 & & 241 \\
\hline $4-7,26-28$ & 38.77 & $1.36 \mathrm{E}-6$ & +40.0 & 202.9 & +23.1 & 227.2 & & 191 \\
\hline $4-7,41-43$ & 38.92 & 1.79 E-6 & +37.2 & 176.6 & +20.6 & 212.4 & & 88 \\
\hline \multicolumn{9}{|l|}{ Hole 575C } \\
\hline $1-1,5-7$ & 0.06 & $4.10 \mathrm{E}-6$ & +34.2 & 307.1 & +32.5 & 286.0 & $\mathrm{~N}$ & 310 \\
\hline $1-1,15-17$ & 0.16 & $2.86 \mathrm{E}-6$ & +24.1 & 314.6 & +19.1 & 291.1 & $\mathrm{~N}$ & 287 \\
\hline $1-1,25-27$ & 0.26 & $1.66 \mathrm{E}-6$ & +32.2 & 305.1 & +28.2 & 284.9 & $\mathrm{~N}$ & 289 \\
\hline $1-1,35-37$ & 0.36 & $1.85 \mathrm{e}-6$ & +19.5 & 304.7 & +26.5 & 287.4 & $\mathrm{~N}$ & 281 \\
\hline $1-1,45-47$ & 0.46 & $4.37 \mathrm{E}-6$ & +16.6 & 294.3 & +6.6 & 274.7 & $\mathrm{~N}$ & 242 \\
\hline $1-1,55-57$ & 0.56 & $4.44 \mathrm{E}-6$ & +18.5 & 300.0 & +17.8 & 284.5 & $\mathrm{~N}$ & 175 \\
\hline $1-1,65-67$ & 0.66 & $5.18 \mathrm{E}-6$ & +19.6 & 300.4 & +12.7 & 276.3 & $\mathrm{~N}$ & 197 \\
\hline $1-1,75-77$ & 0.76 & $4.81 \mathrm{E}-6$ & +21.6 & 291.9 & +13.3 & 274.4 & $\mathrm{~N}$ & 268 \\
\hline $1-1,85-87$ & 0.86 & $5.71 \mathrm{E}-6$ & +19.7 & 307.8 & +11.7 & 286.5 & $\mathrm{~N}$ & 210 \\
\hline $1-1,95-97$ & 0.96 & $5.27 \mathrm{E}-6$ & +20.2 & 338.1 & +20.5 & 307.1 & $\mathrm{~N}$ & 209 \\
\hline $1-1,105-107$ & 1.06 & 8.11 E-6 & +15.1 & 330.6 & +14.1 & 318.0 & $N$ & 203 \\
\hline $1-1,115-117$ & 1.16 & $6.72 \mathrm{E}-6$ & +8.2 & 339.4 & +10.5 & 321.2 & $\mathrm{~N}$ & 194 \\
\hline $1-1,125-127$ & 1.26 & 7.24 E-6 & +6.6 & 337.2 & +8.5 & 326.6 & $\mathrm{~N}$ & 221 \\
\hline $1-1,135-137$ & 1.36 & 8.75 E-6 & +16.3 & 335.2 & +15.7 & 324.5 & $N$ & 227 \\
\hline $1-1,145-147$ & 1.46 & $4.84 \mathrm{E}-6$ & +4.5 & 335.2 & +5.3 & 321.8 & $\mathrm{~N}$ & 183 \\
\hline $1-2,5-7$ & 1.56 & $8.28 \mathrm{E}-6$ & +14.6 & 358.1 & +13.9 & 349.6 & $N$ & 132 \\
\hline $1-2,15-17$ & 1.66 & $4.46 \mathrm{E}-6$ & +12.1 & 5.8 & +13.3 & 349.5 & $\mathrm{~N}$ & 154 \\
\hline $1-2,25-27$ & 1.76 & $2.42 \mathrm{E}-6$ & +11.3 & 352.9 & +14.6 & 342.8 & $N$ & 224 \\
\hline $1-2,35-37$ & 1.86 & $4.46 \mathrm{E}-6$ & +10.1 & 4.7 & +16.4 & 354.1 & $\mathrm{~N}$ & 221 \\
\hline $1-2,45-47$ & 1.96 & $6.70 \mathrm{E}-6$ & +15.8 & 359.7 & +15.7 & 350.7 & $\mathrm{~N}$ & 214 \\
\hline $1-2,55-57$ & 2.06 & 7.26 E-6 & +9.4 & 5.4 & +13.1 & 358.1 & $\mathrm{~N}$ & 179 \\
\hline $1-2,65-67$ & 2.16 & $4.43 \mathrm{E}-6$ & +6.4 & 12.3 & +11.3 & 357.3 & $\mathrm{~N}$ & 157 \\
\hline $1-2,75-77$ & 2.26 & $3.62 \mathrm{E}-6$ & +3.6 & 9.6 & +13.6 & 350.3 & $\mathrm{~N}$ & 194 \\
\hline $1-2,85-87$ & 2.36 & 7.45 E-6 & +5.4 & 356.3 & +11.4 & 349.6 & $\mathrm{~N}$ & 226 \\
\hline $1-2,95-97$ & 2.46 & $7.19 \mathrm{E}-6$ & +9.7 & 358.4 & +21.5 & 346.5 & $\mathrm{~N}$ & 242 \\
\hline $1-2,105-107$ & 2.56 & 2.92 E-6 & -9.1 & 17.8 & +26.1 & 351.8 & $\mathrm{~N}$ & 103 \\
\hline $1-2,115-117$ & 2.66 & $1.83 \mathrm{E}-6$ & -13.8 & 61.4 & +31.5 & 211.5 & R & 50 \\
\hline $1-2,125-127$ & 2.76 & $1.41 \mathrm{E}-6$ & -15.7 & 96.5 & +14.6 & 199.5 & $\mathrm{R}$ & 394 \\
\hline $1-2,135-137$ & 2.86 & $1.73 \mathrm{E}-6$ & -64.4 & 142.4 & -12.3 & 200.4 & $\mathrm{R}$ & 394 \\
\hline $1-2,145-147$ & 2.96 & $1.81 \mathrm{E}-6$ & -33.3 & 192.0 & -2.7 & 215.2 & $\mathrm{R}$ & 418 \\
\hline $1-3,5-7$ & 3.06 & $1.71 \mathrm{E}-6$ & +13.2 & 40.4 & -8.4 & 226.0 & $\mathrm{R}$ & 330 \\
\hline $1-3,15-17$ & 3.16 & $4.84 \mathrm{E}-6$ & +11.6 & 36.0 & +36.5 & 34.1 & $\mathrm{~N}$ & 58 \\
\hline $1-3,25-27$ & 3.26 & $1.08 \mathrm{E}-6$ & -9.2 & 54.1 & -0.1 & 222.1 & $R$ & 413 \\
\hline $1-3,35-37$ & 3.36 & $1.37 \mathrm{E}-6$ & -9.1 & 51.8 & -0.3 & 220.3 & $\mathrm{R}$ & 381 \\
\hline
\end{tabular}


Appendix A. (Continued).

\begin{tabular}{|c|c|c|c|c|c|c|c|c|}
\hline $\begin{array}{l}\text { Core-Section } \\
\text { (interval in } \mathrm{cm} \text { ) }\end{array}$ & $\begin{array}{l}\text { Sub-bottom } \\
\text { depth } \\
\text { (m) }\end{array}$ & $\underset{(\mathrm{G})}{J_{\mathrm{NRM}}}$ & $I_{\mathrm{NRM}}$ & $D_{\mathrm{NRM}}$ & $I_{\text {stable }}$ & $D_{\text {stable }}$ & Polarity & $\begin{array}{l}\text { MDF } \\
\text { (Oe) }\end{array}$ \\
\hline \multicolumn{9}{|l|}{ Hole 575C (Cont.) } \\
\hline $1-3,45-47$ & 3.46 & $1.29 \mathrm{E}-6$ & -57.6 & 71.4 & -17.8 & 222.1 & $\mathbf{R}$ & 450 \\
\hline $1-3,55-57$ & 3.56 & 5.91 E-7 & +2.7 & 64.0 & +2.4 & 220.4 & $\mathbf{R}$ & 629 \\
\hline $1-3,65-67$ & 3.66 & $9.33 \mathrm{E}-7$ & -47.1 & 202.8 & -8.3 & 226.2 & $\mathbf{R}$ & 561 \\
\hline $1-3,75-77$ & 3.76 & 1.13 E-6 & -56.6 & 215.5 & -9.1 & 233.4 & $\mathbf{R}$ & 491 \\
\hline $1-3,85-87$ & 3.86 & $1.08 \mathrm{E}-6$ & -26.4 & 209.2 & -6.5 & 226.2 & R & 466 \\
\hline $1-3,95-97$ & 3.96 & 2.66 E-6 & -13.6 & 215.6 & -6.6 & 221.9 & $\mathbf{R}$ & 374 \\
\hline $1-3,105-107$ & 4.06 & $1.86 \mathrm{E}-6$ & -13.1 & 217.4 & -3.6 & 228.3 & $\mathbf{R}$ & 432 \\
\hline $1-3,115-117$ & 4.16 & $2.66 \mathrm{E}-6$ & -8.7 & 51.5 & +12.0 & 22.6 & $\mathbf{N}$ & 33 \\
\hline $1-3,125-127$ & 4.26 & $7.32 \mathrm{E}-7$ & -48.7 & 132.3 & -5.5 & 232.8 & $\mathbf{R}$ & 478 \\
\hline $1-3,135-137$ & 4.36 & $1.31 \mathrm{E}-6$ & -28.9 & 70.0 & -5.2 & 230.4 & $\mathbf{R}$ & 339 \\
\hline $1-3,145-147$ & 4.46 & 9.68 E-7 & -45.8 & 211.9 & -9.4 & 229.7 & $\mathbf{R}$ & 417 \\
\hline $1-4,5-7$ & 4.56 & 4.20 E-7 & -47.0 & 155.0 & -4.1 & 234.7 & $\mathbf{R}$ & 498 \\
\hline $1-4,15-17$ & 4.66 & 6.27 E-7 & -16.7 & 88.8 & -0.9 & 237.8 & $\mathrm{R}$ & 449 \\
\hline $1-4,25-27$ & 4.76 & 6.67 E-7 & +27.4 & 265.4 & -2.8 & 241.7 & $\mathbf{R}$ & 555 \\
\hline $1-4,35-37$ & 4.86 & $5.49 \mathrm{E}-7$ & -21.0 & 126.9 & -6.4 & 237.0 & $\mathbf{R}$ & 452 \\
\hline $1-4,45-47$ & 4.96 & $1.52 \mathrm{E}-6$ & -19.1 & 222.9 & +1.3 & 241.1 & $\mathbf{R}$ & 424 \\
\hline $1-4,55-57$ & 5.06 & 1.56 E-6 & -25.6 & 227.6 & -4.9 & 233.7 & $\mathrm{R}$ & 411 \\
\hline $1-4,65-67$ & 5.16 & 2.17 E-6 & -22.9 & 226.4 & -5.5 & 235.4 & $\mathbf{R}$ & 413 \\
\hline $1-4,75-77$ & 5.26 & $5.38 \mathrm{E}-7$ & -21.2 & 224.5 & +6.8 & 236.4 & $\mathbf{R}$ & 428 \\
\hline $1-4,85-87$ & 5.36 & 1.21 E-6 & -9.2 & 71.6 & +8.9 & 62.2 & $\mathrm{~N}$ & 109 \\
\hline $1-4,95-97$ & 5.46 & 2.34 E-6 & -2.5 & 72.7 & +13.3 & 61.7 & $\mathrm{~N}$ & 107 \\
\hline $1-4,105-107$ & 5.56 & $1.58 \mathrm{E}-6$ & -5.1 & 71.8 & +11.9 & 61.2 & $\mathrm{~N}$ & 95 \\
\hline $1-4,115-117$ & 5.66 & 2.39 E-6 & -12.5 & 71.7 & +7.9 & 57.2 & $\mathrm{~N}$ & 79 \\
\hline $1-4,125-127$ & 5.76 & 4.45 E-6 & -6.7 & 70.0 & +10.9 & 59.7 & $\mathrm{~N}$ & 84 \\
\hline $1-4,135-137$ & 5.86 & 8.15 E-6 & -4.8 & 76.9 & +10.8 & 65.4 & $\mathrm{~N}$ & 113 \\
\hline $1-4,145-147$ & 5.96 & 9.39 E-6 & -7.5 & 69.6 & +10.6 & 62.4 & $\mathrm{~N}$ & 95 \\
\hline $1-5,15-17$ & 6.16 & 8.85 E-6 & -6.0 & 66.8 & +11.4 & 55.8 & $\mathrm{~N}$ & 86 \\
\hline $1-5,22-24$ & 6.23 & $5.18 \mathrm{E}-6$ & -18.5 & 341.1 & \multicolumn{2}{|c|}{ no stable } & & 174 \\
\hline $2-1,5-7$ & 6.36 & $1.51 \mathrm{E}-6$ & -35.9 & 41.5 & -78.7 & 6.5 & $\mathrm{~N}$ & 56 \\
\hline $2-1,25-27$ & 6.56 & 2.74 E-6 & +3.1 & 6.9 & +7.2 & 1.7 & $\mathrm{~N}$ & 96 \\
\hline $2-1,45-47$ & 6.76 & $1.45 \mathrm{E}-6$ & +51.9 & 345.7 & -7.6 & 199.6 & $\mathbf{R}$ & 47 \\
\hline $2-1,55-57$ & 6.86 & 2.61 E-6 & +40.5 & 6.8 & +17.7 & 195.5 & $\mathbf{R}$ & 30 \\
\hline $2-1,65-67$ & 6.96 & $3.08 \mathrm{E}-6$ & +16.8 & 19.9 & +7.3 & 16.4 & $N$ & 37 \\
\hline $2-1,75-77$ & 7.06 & 2.62 E-6 & +40.2 & 14.9 & +32.9 & 13.6 & $\mathrm{~N}$ & 48 \\
\hline $2-1,85-87$ & 7.16 & 2.47 E-6 & +36.1 & 40.6 & -12.7 & 193.4 & $\mathrm{R}$ & 22 \\
\hline $2-1,95-97$ & 7.26 & 1.92 E-6 & +54.9 & 9.2 & -14.5 & 194.8 & $\mathbf{R}$ & 356 \\
\hline $2-1,105-107$ & 7.36 & $4.53 \mathrm{E}-6$ & -20.3 & 28.2 & -57.7 & 185.5 & $\mathbf{R}$ & 36 \\
\hline $2-1,115-117$ & 7.46 & $5.61 \mathrm{E}-6$ & -13.0 & 21.6 & +6.3 & 2.4 & $\mathrm{~N}$ & 48 \\
\hline $2-1,125-127$ & 7.56 & $5.15 \mathrm{E}-6$ & -10.7 & 25.8 & +9.4 & 11.1 & $\mathrm{~N}$ & 51 \\
\hline $2-1,135-137$ & 7.66 & 4.76 E-6 & -17.7 & 21.8 & +5.0 & 9.4 & $\mathrm{~N}$ & 43 \\
\hline $2-1,145-147$ & 7.76 & 4.84 E-6 & -15.2 & 27.0 & +18.6 & 9.5 & $\mathrm{~N}$ & 42 \\
\hline $2-2,5-7$ & 7.86 & 7.17 E-6 & +13.5 & 356.2 & +2.6 & 1.8 & $\mathrm{~N}$ & 41 \\
\hline $2-2,15-17$ & 7.96 & 3.98 E-6 & +4.3 & 329.3 & -2.4 & 7.2 & $\mathrm{~N}$ & 48 \\
\hline $2-2,25-27$ & 8.06 & 4.20 E-6 & +3.8 & 354.8 & -27.8 & 191.9 & $\mathbf{R}$ & 32 \\
\hline $2-2,35-37$ & 8.16 & 3.01 E-6 & -11.8 & 10.8 & -16.7 & 177.9 & $\mathbf{R}$ & 29 \\
\hline $2-2,45-47$ & 8.26 & 1.96 E-6 & +33.5 & 6.4 & -27.0 & 185.1 & $\mathbf{R}$ & 47 \\
\hline $2-2,55-57$ & 8.36 & 2.04 E-6 & +20.4 & 23.4 & -17.2 & 167.7 & $\mathbf{R}$ & 433 \\
\hline $2-2,65-67$ & 8.46 & $2.06 \mathrm{E}-6$ & +37.1 & 50.3 & -12.4 & 167.8 & $\mathrm{R}$ & 469 \\
\hline $2-2,75-77$ & 8.56 & 9.32 E-7 & +7.0 & 131.1 & -15.6 & 179.7 & $\mathbf{R}$ & 375 \\
\hline $2-2,85-87$ & 8.66 & 1.83 E-6 & +21.2 & 0.6 & -21.8 & 180.2 & $\mathbf{R}$ & 42 \\
\hline $2-2,95-97$ & 8.76 & 1.92 E-6 & +19.4 & 13.5 & -20.3 & 179.7 & $\mathrm{R}$ & 324 \\
\hline $2-2,105-107$ & 8.86 & $2.48 \mathrm{E}-6$ & -2.9 & 18.3 & +5.9 & 168.1 & $\mathbf{R}$ & 34 \\
\hline $2-2,115-117$ & 8.96 & $1.11 \mathrm{E}-6$ & -24.8 & 22.2 & -8.9 & 169.4 & $\mathrm{R}$ & 386 \\
\hline $2-2,125-127$ & 9.06 & 9.84 E-7 & -22.3 & 35.9 & -2.8 & 171.2 & $\mathbf{R}$ & 446 \\
\hline $2-2,135-137$ & 9.16 & $9.72 \mathrm{E}-7$ & -35.4 & 72.1 & -2.9 & 172.4 & $\mathrm{R}$ & 472 \\
\hline $2-2,145-147$ & 9.26 & 6.54 E-7 & -35.3 & 85.1 & -2.8 & 174.8 & $\mathbf{R}$ & 541 \\
\hline $2-3,5-7$ & 9.36 & 2.29 E-6 & +13.6 & 12.2 & -0.2 & 196.1 & $\mathbf{R}$ & 363 \\
\hline $2-3,15-17$ & 9.46 & 2.19 E-6 & +8.8 & 22.2 & +0.7 & 195.1 & $\mathbf{R}$ & 363 \\
\hline $2-3,25-27$ & 9.56 & $2.06 \mathrm{E}-6$ & +2.8 & 10.0 & -0.3 & 200.7 & $\mathbf{R}$ & 382 \\
\hline $2-3,35-37$ & 9.66 & 3.94 E-6 & -5.4 & 21.5 & -6.8 & 196.7 & $\mathbf{R}$ & 29 \\
\hline $2-3,45-47$ & 9.76 & 3.35 E-6 & +39.2 & 355.0 & -4.0 & 200.2 & $\mathbf{R}$ & 29 \\
\hline $2-3,55-57$ & 9.86 & $4.48 \mathrm{E}-6$ & +27.9 & 357.8 & +14.7 & 221.8 & $\mathbf{R}$ & 34 \\
\hline $2-3,65-67$ & 9.96 & 4.71 E-6 & +19.7 & 351.7 & +3.5 & 345.4 & $\mathrm{~N}$ & 43 \\
\hline $2-3,75-77$ & 10.06 & $5.12 \mathrm{E}-6$ & +26.7 & 6.7 & +24.0 & 359.4 & $\mathrm{~N}$ & 48 \\
\hline $2-3,85-87$ & 10.16 & $4.18 \mathrm{E}-6$ & +33.1 & 0.4 & +33.0 & 356.1 & $\mathrm{~N}$ & 37 \\
\hline $2-3,95-97$ & 10.26 & 4.74 E-6 & +22.7 & 1.5 & +13.7 & 355.7 & $\mathbf{N}$ & 48 \\
\hline $2-3,105-107$ & 10.36 & $4.58 \mathrm{E}-6$ & +1.6 & 12.5 & +13.8 & 356.5 & $\mathrm{~N}$ & 44 \\
\hline $2-3,115-117$ & 10.46 & $5.63 \mathrm{E}-6$ & +0.1 & 4.1 & +13.3 & 350.3 & $\mathbf{N}$ & 49 \\
\hline $2-3,125-127$ & 10.56 & $5.80 \mathrm{E}-6$ & +2.4 & 357.9 & +12.5 & 351.9 & $\mathrm{~N}$ & 51 \\
\hline $2-3,135-137$ & 10.66 & $4.56 \mathrm{E}-6$ & +6.3 & 359.0 & +19.7 & 349.3 & $\mathrm{~N}$ & 47 \\
\hline $2-3,145-147$ & 10.76 & $3.25 \mathrm{E}-6$ & +1.2 & 345.1 & +11.7 & 335.2 & $\mathrm{~N}$ & 41 \\
\hline $2-4,5-7$ & 10.86 & $4.01 \mathrm{E}-6$ & +16.0 & 10.2 & +23.0 & 3.3 & $\mathrm{~N}$ & 35 \\
\hline $2-4,15-17$ & 10.96 & $3.38 \mathrm{E}-6$ & +10.9 & 5.3 & -3.5 & 196.4 & $\mathbf{R}$ & 28 \\
\hline $2-4,25-27$ & 11.06 & $2.39 \mathrm{E}-6$ & +19.6 & 359.7 & +3.5 & 200.8 & $\mathbf{R}$ & 293 \\
\hline
\end{tabular}


Appendix A. (Continued).

\begin{tabular}{|c|c|c|c|c|c|c|c|c|}
\hline $\begin{array}{c}\text { Core-Section } \\
\text { (interval in cm) }\end{array}$ & $\begin{array}{l}\text { Sub-bottom } \\
\text { depth } \\
\text { (m) }\end{array}$ & $\begin{array}{c}J_{\text {NRM }} \\
(\mathrm{G})\end{array}$ & $I_{\mathrm{NRM}}$ & $D_{\text {NRM }}$ & $I_{\text {stable }}$ & $D_{\text {stable }}$ & Polarity & $\begin{array}{l}\text { MDF } \\
\text { (Oe) }\end{array}$ \\
\hline \multicolumn{9}{|l|}{ Hole $575 \mathrm{C}$ (Cont.) } \\
\hline $2-4,35-37$ & 11.16 & $3.47 \mathrm{E}-6$ & +21.9 & 358.4 & +6.4 & 201.2 & $\mathrm{R}$ & 30 \\
\hline $2-4,45-47$ & 11.26 & $1.27 \mathrm{E}-6$ & +22.9 & 286.0 & +2.9 & 191.0 & $\mathbf{R}$ & 293 \\
\hline $2-4,55-57$ & 11.36 & $2.41 \mathrm{E}-6$ & +62.7 & 320.4 & +10.6 & 191.3 & $\mathbf{R}$ & 258 \\
\hline $2-4,65-67$ & 11.46 & $3.66 \mathrm{E}-6$ & +42.5 & 348.6 & -2.8 & 204.3 & $\mathrm{R}$ & 32 \\
\hline $2-4,75-77$ & 11.56 & $4.72 \mathrm{E}-6$ & +35.5 & 356.6 & +42.0 & 345.4 & $\mathrm{~N}$ & 41 \\
\hline $2-4,85-87$ & 11.66 & $4.93 \mathrm{E}-6$ & +20.7 & 342.5 & +13.0 & 347.6 & $\mathrm{~N}$ & 49 \\
\hline $2-4,95-97$ & 11.76 & $5.69 \mathrm{E}-6$ & +23.2 & 354.0 & +21.2 & 2.8 & $\mathrm{~N}$ & 61 \\
\hline $2-4,105-107$ & 11.86 & 7.48 E-6 & -6.9 & 281.3 & -8.5 & 243.6 & $\mathrm{~N}$ & 897 \\
\hline $2-4,115-117$ & 11.96 & $5.11 \mathrm{E}-6$ & +5.8 & 1.6 & +17.9 & 346.6 & $\mathrm{~N}$ & 44 \\
\hline $2-4,125-127$ & 12.06 & $5.09 \mathrm{E}-6$ & -24.1 & 338.4 & \multicolumn{2}{|c|}{ no stable } & & 91 \\
\hline $2-5,5-7$ & 12.36 & $6.09 \mathrm{E}-6$ & -9.8 & 359.2 & +13.6 & 343.4 & $\mathrm{~N}$ & 49 \\
\hline $2-5,15-17$ & 12.46 & $5.41 \mathrm{e}-6$ & +9.4 & 350.3 & +14.6 & 340.8 & $\mathrm{~N}$ & 54 \\
\hline $2-5,25-27$ & 12.56 & 5.64 E-6 & +9.2 & 350.1 & +15.7 & 340.4 & $\mathrm{~N}$ & 61 \\
\hline $2-5,35-37$ & 12.66 & $3.85 \mathrm{E}-6$ & +7.2 & 353.9 & +21.0 & 312.6 & $\mathrm{~N}$ & 41 \\
\hline $2-5,45-47$ & 12.76 & 2.47 E-6 & +13.6 & 313.4 & +14.7 & 215.6 & $\mathbf{R}$ & 40 \\
\hline $2-5,55-57$ & 12.86 & 2.42 E-6 & +44.0 & 337.2 & +14.5 & 180.1 & $\mathbf{R}$ & 34 \\
\hline $2-5,65-67$ & 12.96 & $1.98 \mathrm{E}-6$ & +43.6 & 330.9 & +16.2 & 173.0 & $\mathbf{R}$ & 42 \\
\hline $2-5,75-77$ & 13.06 & $1.48 \mathrm{E}-6$ & +43.7 & 332.2 & +11.8 & 173.8 & $\mathbf{R}$ & 46 \\
\hline $2-5,85-87$ & 13.16 & $1.90 \mathrm{E}-6$ & +42.6 & 335.9 & +18.3 & 172.0 & $\mathbf{R}$ & 36 \\
\hline $2-5,95-97$ & 13.26 & $1.29 \mathrm{E}-6$ & +27.0 & 333.1 & -2.7 & 186.2 & $\mathbf{R}$ & 34 \\
\hline $2-5,105-107$ & 13.36 & $6.36 \mathrm{E}-7$ & -16.8 & 33.9 & -6.5 & 166.5 & $\mathrm{R}$ & 455 \\
\hline $2-5,115-117$ & 13.46 & $7.46 \mathrm{E}-7$ & +3.8 & 66.4 & +6.7 & 164.8 & $\mathbf{R}$ & 494 \\
\hline $2-5,125-127$ & 13.56 & $9.29 \mathrm{E}-7$ & -7.2 & 12.6 & -5.7 & 173.6 & $\mathbf{R}$ & 34 \\
\hline $2-5,135-137$ & 13.66 & $8.16 \mathrm{E}-7$ & +2.6 & 31.2 & +3.6 & 168.1 & $\mathbf{R}$ & 378 \\
\hline $2-5,145-147$ & 13.76 & $1.90 \mathrm{E}-6$ & +0.3 & 357.2 & +32.1 & 190.8 & $\mathbf{R}$ & 42 \\
\hline $2-6,5-7$ & 13.86 & $3.04 \mathrm{E}-6$ & +21.2 & 345.4 & -7.9 & 175.5 & $\mathrm{R}$ & 30 \\
\hline $2-6,15-17$ & 13.96 & $1.99 \mathrm{E}-6$ & +12.4 & 345.1 & -0.8 & 173.8 & R & 29 \\
\hline $2-6,25-27$ & 14.06 & $1.32 \mathrm{E}-6$ & +7.9 & 349.2 & -2.4 & 171.0 & $\mathbf{R}$ & 28 \\
\hline $2-6,35-37$ & 14.16 & $1.58 \mathrm{E}-6$ & +17.5 & 344.2 & +14.1 & 176.8 & R & 30 \\
\hline $2-6,45-47$ & 14.26 & $1.54 \mathrm{E}-6$ & +0.4 & 357.7 & -3.8 & 173.2 & $\mathbf{R}$ & 34 \\
\hline $2-6,55-57$ & 14.36 & $1.53 \mathrm{E}-6$ & +12.8 & 0.8 & +20.9 & 171.9 & R & 35 \\
\hline $2-6,65-67$ & 14.46 & $8.90 \mathrm{E}-7$ & +1.0 & 346.4 & \multicolumn{2}{|c|}{ no stable } & & 42 \\
\hline $2-6,75-77$ & 14.56 & $6.84 \mathrm{E}-7$ & +9.1 & 347.7 & +12.8 & 179.5 & $\mathrm{R}$ & 34 \\
\hline $2-6,85-87$ & 14.66 & $7.30 \mathrm{E}-7$ & +8.9 & 345.1 & +16.2 & 194.3 & $\mathrm{R}$ & 39 \\
\hline $2-6,95-97$ & 14.76 & $5.82 \mathrm{E}-7$ & +12.1 & 353.5 & +59.2 & 215.9 & $\mathbf{R}$ & 40 \\
\hline $2-6,105-107$ & 14.86 & $5.51 \mathrm{E}-7$ & +6.1 & 351.1 & +31.6 & 208.0 & R & 43 \\
\hline $2-6,115-117$ & 14.96 & $5.46 \mathrm{E}-7$ & -8.0 & 350.4 & -19.7 & 148.5 & $\mathrm{R}$ & 30 \\
\hline $2-6,125-127$ & 15.06 & $5.33 \mathrm{E}-7$ & -1.8 & 19.2 & -3.1 & 142.7 & $\mathbf{R}$ & 38 \\
\hline $2-5,135-137$ & 15.16 & $3.40 \mathrm{E}-7$ & +32.0 & 5.5 & +9.7 & 179.9 & $\mathbf{R}$ & 369 \\
\hline $2-6,145-147$ & 15.26 & $7.58 \mathrm{E}-7$ & +9.6 & 3.8 & +43.2 & 218.8 & $\mathbf{R}$ & 43 \\
\hline $2-7,5-7$ & 15.36 & 3.70 E-7 & +38.1 & 30.9 & +22.8 & 210.1 & $\mathrm{R}$ & 298 \\
\hline $2-7,15-17$ & 15.46 & $4.36 \mathrm{E}-7$ & +12.7 & 34.1 & +6.9 & 231.6 & $\mathbf{R}$ & 39 \\
\hline $2-7,25-27$ & 15.56 & $5.84 \mathrm{E}-7$ & +13.9 & 37.2 & +40.9 & 293.2 & $\mathrm{~N}$ & 49 \\
\hline $2-7,35-37$ & 15.66 & $5.59 \mathrm{E}-7$ & -4.3 & 73.1 & +13.8 & 27.3 & $\mathrm{~N}$ & 61 \\
\hline
\end{tabular}

Notes: $J=$ intensity; $I=$ inclination, $D=$ declination $(+=$ downward, $-=$ upward $)$; polarity is as derived from stable inclinations and declinations $(\mathrm{N}=$ normal, $\mathrm{R}=$ reversed). 
APPENDIX B

Paleomagnetic Data Derived from Oligocene to Late Miocene Leg 85 Samples (samples were measured at HIG, after demagnetization at $50 \mathrm{Oe}$ )

\begin{tabular}{crrr}
\hline $\begin{array}{c}\text { Sub-bottom } \\
\text { depth } \\
(\mathrm{m})\end{array}$ & $\begin{array}{c}\text { Declination } \\
\left({ }^{\circ}\right)\end{array}$ & $\begin{array}{c}\text { Inclination } \\
\left({ }^{\circ}\right)\end{array}$ & $\begin{array}{c}\text { Intensity } \\
(\mathrm{G})\end{array}$ \\
\hline Hole 572A & & & \\
& & & \\
120.00 & -30.75 & -33.14 & 0.4140 E-6 \\
121.11 & -9.93 & 7.01 & 0.1202 E-5 \\
122.61 & -8.75 & 27.17 & 0.7416 E-6 \\
124.11 & -32.03 & -13.53 & 0.6314 E-6 \\
125.61 & -11.06 & -0.03 & 0.7462 E-6 \\
127.11 & -84.24 & -13.65 & 0.9357 E-6 \\
129.17 & -24.49 & -16.97 & 0.6278 E-6 \\
130.33 & -16.79 & -17.00 & 0.6634 E-6 \\
131.81 & -176.09 & -26.31 & 0.3915 E-5 \\
133.31 & 2.04 & -3.32 & 0.9725 E-6 \\
134.81 & -6.53 & 6.61 & 0.9195 E-6 \\
136.31 & -15.47 & 3.35 & 0.6878 E-6 \\
139.09 & -14.29 & 19.42 & 0.1012 E-5 \\
140.59 & -2.18 & 12.06 & 0.5413 E-6 \\
142.09 & 48.59 & -6.87 & 0.2807 E-5 \\
143.59 & -9.26 & 9.35 & 0.6088 E-6 \\
145.09 & -25.83 & 6.86 & 0.7711 E-6 \\
145.80 & -79.61 & 21.75 & 0.8132 E-5 \\
147.30 & -20.03 & -27.25 & 0.9364 E-6 \\
148.80 & -1.25 & 8.03 & 0.9587 E-6 \\
150.30 & -20.42 & -12.81 & 0.7407 E-6 \\
151.80 & -133.48 & -17.92 & 0.6038 E-6 \\
153.30 & -16.85 & 17.63 & 0.9806 E-6
\end{tabular}

Appendix B. (Continued).

\begin{tabular}{cccc}
\hline $\begin{array}{c}\text { Sub-bottom } \\
\text { depth } \\
(\mathrm{m})\end{array}$ & $\begin{array}{c}\text { Declination } \\
\left({ }^{\circ}\right)\end{array}$ & $\begin{array}{c}\text { Inclination } \\
\left({ }^{\circ}\right)\end{array}$ & $\begin{array}{c}\text { Intensity } \\
(\mathrm{G})\end{array}$ \\
\hline
\end{tabular}

Hole 572C (Cont.)

\begin{tabular}{|c|c|c|c|}
\hline 132.19 & -32.58 & -35.89 & 0.4285 E-6 \\
\hline 132.69 & -22.28 & -17.31 & 0.6186 E-6 \\
\hline 133.19 & -11.94 & -28.12 & 0.8788 E-6 \\
\hline 133.69 & -20.07 & 2.97 & 0.8102 E-6 \\
\hline 134.19 & -16.21 & 3.71 & 0.7840 E-6 \\
\hline 134.69 & -18.81 & -18.69 & 0.8381 E-6 \\
\hline 135.19 & -25.23 & 0.56 & 0.1240 E-5 \\
\hline 135.69 & -32.16 & -19.57 & 0.6772 E-6 \\
\hline 136.19 & -11.76 & -19.97 & 0.1198 E-5 \\
\hline 136.69 & -5.75 & -30.89 & 0.7144 E-6 \\
\hline 137.19 & -52.12 & 62.14 & 0.1279 E-5 \\
\hline 137.69 & -11.48 & -2.73 & 0.7534 E-6 \\
\hline 138.19 & -11.70 & -4.61 & 0.1042 E-5 \\
\hline 138.69 & -33.83 & 25.88 & 0.5139 E-6 \\
\hline 139.19 & -11.53 & 7.91 & 0.9498 E-6 \\
\hline 139.69 & -11.38 & -4.01 & 0.9837 E-6 \\
\hline 140.77 & -4.19 & -8.91 & 0.9177 E-6 \\
\hline 140.80 & -12.22 & 1.04 & 0.8669 E-6 \\
\hline 141.29 & -7.29 & 20.13 & 0.8724 E-6 \\
\hline 142.30 & -13.14 & 3.52 & 0.7377 E-6 \\
\hline 142.79 & -2.13 & -10.84 & 0.8356 E-6 \\
\hline 143.28 & -13.44 & 4.43 & 0.7186 E-6 \\
\hline 144.00 & -10.69 & -6.76 & 0.9705 E-6 \\
\hline 144.70 & -28.97 & -2.50 & $0.5550 \mathrm{E}-6$ \\
\hline 145.10 & -12.16 & 3.56 & 0.8993 E-6 \\
\hline 145.62 & -6.63 & -4.89 & 0.9526 E-6 \\
\hline 150.73 & -8.89 & -0.78 & 0.1100 E-5 \\
\hline 151.35 & -10.10 & 2.13 & 0.8162 E-6 \\
\hline 151.89 & -38.46 & 63.84 & 0.8778 E-6 \\
\hline 152.39 & -12.47 & 15.45 & 0.6561 E-6 \\
\hline 152.85 & 36.43 & -51.98 & 0.5028 E-6 \\
\hline 153.39 & -12.34 & -9.72 & 0.7096 E-6 \\
\hline 153.89 & -10.87 & 7.47 & 0.9266 E-6 \\
\hline 154.35 & -2.11 & 5.25 & 0.1255 E-5 \\
\hline 154.89 & -12.30 & -23.57 & 0.1481 E- 5 \\
\hline 155.39 & -7.84 & 12.71 & 0.7945 E-6 \\
\hline 155.85 & -19.01 & -28.72 & 0.4982 E-6 \\
\hline 156.39 & -24.48 & -28.81 & 0.8046 E-6 \\
\hline 156.89 & -16.39 & -37.02 & 0.5728 E-6 \\
\hline 157.35 & -8.40 & 2.59 & 0.1009 E-5 \\
\hline 157.70 & -45.08 & -26.67 & 0.2735 E-6 \\
\hline 158.25 & -4.34 & -14.06 & 0.8719 E-6 \\
\hline 158.61 & -167.35 & -39.02 & 0.7069 E-6 \\
\hline 163.89 & -14.38 & -3.12 & 0.1297 E-5 \\
\hline 164.24 & -17.10 & -16.14 & 0.1020 E-5 \\
\hline 164.65 & -39.85 & -13.52 & 0.5896 E-6 \\
\hline 165.01 & -18.33 & -17.82 & 0.9418 E-6 \\
\hline 165.48 & -13.68 & -16.83 & 0.8960 E-6 \\
\hline 165.92 & -12.09 & -32.99 & 0.9920 E-6 \\
\hline 166.33 & -10.31 & 14.86 & 0.7394 E-6 \\
\hline 166.85 & -49.97 & 1.01 & 0.1625 E-5 \\
\hline 167.35 & -41.09 & -1.07 & 0.1269 E-5 \\
\hline 167.83 & -11.07 & -6.81 & 0.8448 E-6 \\
\hline 168.24 & -33.38 & -1.47 & $0.6688 \mathrm{E}-6$ \\
\hline \multicolumn{4}{|c|}{ Iole 572D } \\
\hline 155.21 & -3.82 & 53.57 & 0.3624 E-5 \\
\hline 155.71 & -38.52 & -37.92 & 0.4579 E-6 \\
\hline 160.70 & -15.98 & -31.89 & 0.1086 E-5 \\
\hline 161.41 & -11.58 & -8.63 & 0.5493 E-6 \\
\hline 166.46 & -8.68 & -14.22 & 0.7600 E-6 \\
\hline 167.33 & -18.01 & -23.65 & 0.7482 E-6 \\
\hline 170.15 & 5.01 & -14.65 & 0.2276 E-5 \\
\hline 174.88 & -17.08 & -14.75 & 0.7485 E-6 \\
\hline 179.61 & -14.80 & -13.99 & 0.3772 E- 6 \\
\hline 195.17 & -15.52 & -27.21 & 0.8771 E-6 \\
\hline 196.56 & -31.45 & 26.45 & 0.4452 E-6 \\
\hline 206.71 & -18.77 & -30.71 & 0.5987 E-6 \\
\hline 207.29 & -16.08 & -13.08 & 0.5534 E-6 \\
\hline
\end{tabular}


Appendix B. (Continued).

\begin{tabular}{cccc}
\hline $\begin{array}{c}\text { Sub-bottom } \\
\text { depth } \\
(\mathrm{m})\end{array}$ & $\begin{array}{c}\text { Declination } \\
\left({ }^{\circ}\right)\end{array}$ & $\begin{array}{c}\text { Inclination } \\
\left({ }^{\circ}\right)\end{array}$ & Intensity \\
& & $(\mathrm{G})$
\end{tabular}

Hole 572D (Cont.)

\begin{tabular}{|c|c|c|c|}
\hline 207.81 & -16.52 & -20.30 & 0.6360 E-6 \\
\hline 209.85 & -9.52 & -24.14 & 0.7813 E-6 \\
\hline 210.81 & -7.75 & -1.15 & 0.5789 E- 6 \\
\hline 211.85 & -2.28 & -21.83 & 0.7976 E-6 \\
\hline 212.43 & -12.73 & -5.40 & 0.6967 E-6 \\
\hline 213.17 & -17.53 & -18.95 & 0.8165 E-6 \\
\hline 215.20 & -22.97 & 18.59 & 0.4700 E-6 \\
\hline 215.91 & -14.07 & -7.39 & 0.6630 E-6 \\
\hline 216.59 & -13.90 & -36.75 & 0.8534 E-6 \\
\hline 217.70 & -67.48 & -15.51 & 0.1477 E-5 \\
\hline 218.90 & -20.94 & -8.88 & 0.5221 E-6 \\
\hline 219.58 & -14.39 & -21.04 & 0.8489 E-6 \\
\hline 221.33 & -1.23 & -42.77 & 0.3744 E-6 \\
\hline 221.75 & -13.96 & -29.20 & 0.5950 E-6 \\
\hline 222.96 & -33.83 & -27.70 & 0.3443 E-6 \\
\hline 224.27 & -20.36 & -34.81 & 0.6797 E-6 \\
\hline 224.78 & -16.39 & -7.72 & $0.7839 \mathrm{E}-6$ \\
\hline 225.57 & -9.91 & -35.13 & 0.5328 E-6 \\
\hline 226.00 & -68.72 & 9.64 & 0.1311 E-5 \\
\hline 226.42 & -9.87 & -14.00 & 0.5632 E-6 \\
\hline 227.26 & 8.46 & -19.50 & 0.7741 E-6 \\
\hline 227.71 & -22.81 & -22.63 & 0.7773 E-6 \\
\hline 228.82 & 12.18 & -29.71 & $0.6925 \mathrm{E}-6$ \\
\hline 229.67 & -20.08 & -23.08 & 0.9788 E-6 \\
\hline 230.61 & -18.42 & -18.40 & 0.7743 E-6 \\
\hline 231.25 & -8.98 & -21.49 & 0.6186 E-6 \\
\hline 231.75 & -19.82 & 0.46 & 0.4861 E-6 \\
\hline 236.61 & -20.27 & -4.66 & 0.6795 E-6 \\
\hline 237.34 & 0.12 & -15.09 & 0.6262 E-6 \\
\hline 238.84 & -14.81 & -16.79 & 0.6937 E-6 \\
\hline 240.73 & -11.52 & -13.60 & 0.4501 E-6 \\
\hline 241.30 & -25.25 & -22.29 & 0.7709 E-6 \\
\hline 241.84 & -16.91 & -15.83 & 0.5727 E-6 \\
\hline 242.78 & 0.39 & 21.02 & 0.7595 E-6 \\
\hline 243.58 & -11.07 & 16.97 & 0.6840 E-6 \\
\hline 244.41 & 34.50 & -18.63 & 0.3768 E-6 \\
\hline 245.35 & -22.48 & -19.93 & 0.5722 E-6 \\
\hline 245.78 & -19.38 & -25.11 & 0.5653 E-6 \\
\hline 246.05 & -42.82 & -7.09 & 0.6969 E-6 \\
\hline 247.03 & -26.75 & -18.28 & 0.4328 E-6 \\
\hline 248.38 & -90.44 & 1.06 & $0.8393 \mathrm{E}-4$ \\
\hline 249.69 & 1.80 & -2.46 & 0.8809 E-6 \\
\hline 250.87 & -7.92 & 25.23 & 0.8604 E-6 \\
\hline 251.66 & -10.69 & 24.56 & 0.5116 E-6 \\
\hline 252.68 & -159.02 & 39.25 & 0.2302 E- 6 \\
\hline 253.45 & -26.03 & -14.16 & 0.7069 E-6 \\
\hline 254.18 & 10.47 & -19.19 & $0.1020 \mathrm{E}-5$ \\
\hline 254.90 & -3.75 & -2.36 & 0.4406 E-6 \\
\hline 255.47 & -22.86 & -15.24 & 0.7378 E-6 \\
\hline 255.67 & -6.19 & -26.16 & 0.9569 E-6 \\
\hline 256.75 & -0.07 & -5.65 & 0.1362 E-4 \\
\hline 257.21 & -60.13 & -28.01 & 0.9167 E-5 \\
\hline 257.82 & 157.88 & -17.59 & $0.1356 \mathrm{E}-4$ \\
\hline 258.25 & -1.74 & -25.32 & 0.8024 E-6 \\
\hline 259.29 & -16.69 & -11.11 & 0.6727 E- 6 \\
\hline 260.21 & 33.21 & 4.53 & 0.2305 E-5 \\
\hline 261.12 & 7.33 & -4.43 & 0.7210 E-5 \\
\hline 265.63 & 37.35 & -7.17 & 0.1404 E-5 \\
\hline 267.51 & -0.35 & -21.99 & 0.4359 E-6 \\
\hline 267.61 & 14.02 & 5.31 & 0.1126 E-5 \\
\hline 268.40 & -7.66 & -15.32 & 0.9872 E-6 \\
\hline 270.72 & 17.49 & -51.30 & 0.6518 E-5 \\
\hline 272.32 & -3.35 & 0.12 & 0.1596 E-5 \\
\hline 277.35 & 83.04 & 10.26 & 0.3377 E-4 \\
\hline 279.31 & -24.17 & 2.89 & $0.1902 \mathrm{E}-4$ \\
\hline 281.15 & -140.64 & -1.92 & $0.1147 \mathrm{E}-4$ \\
\hline 281.74 & 9.48 & -7.15 & $0.2453 \mathrm{E}-4$ \\
\hline 284.14 & 4.36 & -5.18 & 0.1032 E-5 \\
\hline 287.41 & -0.23 & -29.87 & $0.9808 \mathrm{E}-6$ \\
\hline 294.08 & -152.02 & 4.50 & 0.6370 E-5 \\
\hline 295.23 & 79.83 & -1.39 & $0.2974 \mathrm{E}-4$ \\
\hline 296.20 & -47.31 & -8.45 & $0.2746 \mathrm{E}-4$ \\
\hline 296.71 & 59.33 & 6.36 & 0.2152 E-4 \\
\hline 297.24 & 179.33 & -33.50 & 0.3539 E- 5 \\
\hline
\end{tabular}

Appendix B. (Continued).

\begin{tabular}{cccc}
$\begin{array}{c}\text { Sub-bottom } \\
\text { depth } \\
(\mathrm{m})\end{array}$ & $\begin{array}{c}\text { Declination } \\
\left({ }^{\circ}\right)\end{array}$ & $\begin{array}{c}\text { Inclination } \\
\left({ }^{\circ}\right)\end{array}$ & $\begin{array}{c}\text { Intensity } \\
(\mathrm{G})\end{array}$ \\
\hline
\end{tabular}

\section{Hole 572D (Cont.)}

\begin{tabular}{|c|c|c|c|}
\hline 297.76 & 88.49 & -0.95 & $0.2232 \mathrm{E}-4$ \\
\hline 298.21 & 37.56 & -8.58 & $0.1265 \mathrm{E}-4$ \\
\hline 299.17 & -10.25 & -13.36 & 0.7184 E-6 \\
\hline 299.75 & -0.23 & -7.25 & 0.6949 E-6 \\
\hline 300.56 & 14.08 & -37.07 & 0.8316 E-6 \\
\hline 301.25 & 42.67 & -2.65 & 0.2334 E-5 \\
\hline 302.73 & -6.59 & -24.36 & 0.7178 E-6 \\
\hline 303.27 & -2.32 & 4.13 & 0.1181 E-5 \\
\hline 304.16 & -1.69 & -42.06 & 0.7279 E-6 \\
\hline 304.77 & -20.14 & -49.43 & 0.6524 E-6 \\
\hline 306.20 & 71.75 & -33.87 & 0.9729 E-6 \\
\hline 312.82 & -11.89 & 12.27 & 0.5565 E-6 \\
\hline 313.82 & -2.27 & -17.47 & 0.6279 E-6 \\
\hline 314.45 & -6.03 & -13.49 & 0.6298 E-6 \\
\hline 314.93 & -14.27 & -24.38 & 0.5363 E- 6 \\
\hline 315.94 & -10.78 & -12.21 & 0.5764 E-6 \\
\hline 316.54 & -12.43 & -12.81 & 0.5911 E-6 \\
\hline 317.44 & -16.07 & -23.90 & 0.5329 E-6 \\
\hline 318.94 & -17.59 & -26.50 & 0.5720 E-6 \\
\hline 319.59 & -2.07 & 3.86 & 0.4699 E-6 \\
\hline 320.44 & -9.79 & -6.63 & 0.6976 E-6 \\
\hline 322.40 & -19.62 & -16.64 & $0.6988 \mathrm{E}-6$ \\
\hline 324.25 & 0.18 & -39.53 & 0.6960 E-6 \\
\hline 332.60 & 149.09 & -35.53 & 0.2580 E- 5 \\
\hline 333.68 & 6.39 & -19.14 & 0.6672 E-5 \\
\hline 334.66 & 14.93 & -58.26 & 0.5477 E-5 \\
\hline 350.76 & 4.80 & -67.87 & 0.8096 E-6 \\
\hline 351.90 & -2.52 & -48.04 & 0.9978 E-6 \\
\hline 353.40 & -16.92 & -42.49 & 0.1132 E-5 \\
\hline 353.77 & -5.51 & -48.53 & 0.1057 E-5 \\
\hline 354.51 & 1.20 & -42.32 & 0.9772 E-6 \\
\hline 360.77 & -31.21 & -46.29 & 0.8072 E-6 \\
\hline 360.78 & -37.95 & -41.04 & $0.5761 \mathrm{E}-6$ \\
\hline 361.42 & -30.14 & -31.41 & 0.4309 E-6 \\
\hline 361.76 & -11.99 & -21.42 & 0.6691 E-6 \\
\hline 362.85 & -94.32 & -25.47 & $0.2501 \mathrm{E}-5$ \\
\hline 363.30 & -149.29 & -22.49 & 0.6058 E- 5 \\
\hline 363.73 & -50.64 & 5.85 & 0.5503 E-5 \\
\hline 364.38 & -113.67 & 2.46 & 0.1272 E- 4 \\
\hline 364.63 & 37.70 & -11.31 & $0.1325 \mathrm{E}-4$ \\
\hline 365.27 & 41.58 & -13.36 & 0.6311 E-5 \\
\hline 365.74 & -4.31 & -31.31 & 0.6006 E-5 \\
\hline 366.21 & -17.64 & -25.08 & 0.3020 E-5 \\
\hline 367.39 & -101.55 & -16.96 & 0.1570 E-5 \\
\hline 369.85 & 101.21 & -17.11 & 0.6478 E-5 \\
\hline 370.36 & 85.91 & 19.77 & $0.1123 \mathrm{E}-4$ \\
\hline 370.81 & 176.37 & 12.82 & 0.1045 E-4 \\
\hline 371.15 & -45.87 & -2.95 & $0.2160 \mathrm{E}-4$ \\
\hline 372.46 & 58.66 & 3.92 & 0.7981 E-5 \\
\hline 372.52 & 105.06 & -2.49 & 0.1124 E-4 \\
\hline 373.47 & -154.67 & -3.41 & 0.1896 E-4 \\
\hline 375.37 & -129.14 & -8.00 & 0.1711 E-4 \\
\hline 376.23 & 125.76 & 5.12 & $0.2589 \mathrm{E}-4$ \\
\hline 376.37 & -156.34 & 2.18 & $0.3179 \mathrm{E}-4$ \\
\hline 377.33 & 61.67 & 5.57 & 0.7119 E-5 \\
\hline 377.98 & 54.95 & -5.27 & 0.4237 E-4 \\
\hline 379.46 & -18.95 & 4.64 & 0.1736 E-4 \\
\hline 380.06 & 19.66 & 5.84 & $0.2397 \mathrm{E}-4$ \\
\hline 381.36 & 71.52 & -12.66 & 0.1707 E-4 \\
\hline 381.89 & 125.02 & -6.01 & 0.4127 E-5 \\
\hline 382.99 & -82.29 & -11.77 & $0.1304 \mathrm{E}-4$ \\
\hline 383.39 & 116.50 & 0.92 & 0.7968 E-5 \\
\hline 383.83 & 73.63 & -15.91 & 0.3317 E-4 \\
\hline 384.96 & 22.98 & 7.39 & 0.3002 E-4 \\
\hline 385.39 & 135.63 & -72.27 & 0.3861 E-5 \\
\hline 386.21 & 130.77 & -9.36 & 0.1759 E-4 \\
\hline 386.59 & 162.53 & 8.45 & 0.2731 E-4 \\
\hline 387.85 & -150.65 & -4.91 & 0.2652 E-4 \\
\hline 388.27 & 89.50 & -17.95 & $0.2685 \mathrm{E}-4$ \\
\hline 388.93 & -144.08 & 0.62 & $0.2755 \mathrm{E}-4$ \\
\hline 389.45 & -24.05 & -22.78 & 0.6259 E-5 \\
\hline 389.96 & -156.88 & -20.57 & $0.2103 \mathrm{E}-4$ \\
\hline 390.26 & 177.93 & -6.46 & $0.1528 \mathrm{E}-4$ \\
\hline 390.82 & -170.43 & -2.12 & 0.1305 E-4 \\
\hline
\end{tabular}


Appendix B. (Continued).

\begin{tabular}{cccc}
\hline $\begin{array}{c}\text { Sub-bottom } \\
\text { depth } \\
(\mathrm{m})\end{array}$ & $\begin{array}{c}\text { Declination } \\
\left({ }^{\circ}\right)\end{array}$ & $\begin{array}{c}\text { Inclination } \\
\left({ }^{\circ}\right)\end{array}$ & $\begin{array}{c}\text { Intensity } \\
(\mathrm{G})\end{array}$ \\
\hline
\end{tabular}

Hole 572D (Cont.)

\begin{tabular}{|c|c|c|c|}
\hline 391.46 & -89.16 & -12.73 & $0.2910 \mathrm{E}-4$ \\
\hline 391.60 & -11.69 & -11.06 & $0.2916 \mathrm{E}-4$ \\
\hline 392.47 & -5.17 & -16.95 & 0.5982 E- 5 \\
\hline 392.81 & -42.85 & -20.42 & $0.1181 \mathrm{E}-4$ \\
\hline 393.41 & 168.78 & -72.74 & 0.5074 E-5 \\
\hline 393.98 & 74.84 & -9.07 & 0.1589 E-4 \\
\hline 394.44 & 57.33 & -0.95 & $0.2508 \mathrm{E}-4$ \\
\hline 396.03 & -50.87 & -7.89 & $0.2516 \mathrm{E}-4$ \\
\hline 396.31 & -62.94 & -26.79 & 0.4312 E- 5 \\
\hline 396.55 & -39.29 & -49.79 & 0.3632 E-5 \\
\hline 397.45 & -40.25 & -60.94 & 0.1318 E-5 \\
\hline 398.24 & -140.23 & 3.26 & $0.1535 \mathrm{E}-4$ \\
\hline 398.69 & -62.93 & 11.04 & $0.1686 \mathrm{E}-4$ \\
\hline 399.14 & 150.05 & 17.28 & $0.1288 \mathrm{E}-4$ \\
\hline 399.76 & -130.77 & 11.69 & $0.1786 \mathrm{E}-4$ \\
\hline 400.31 & 98.51 & -6.22 & 0.1764 E-4 \\
\hline 400.73 & 23.20 & -10.12 & $0.2191 \mathrm{E}-4$ \\
\hline 401.22 & -6.49 & -2.11 & $0.2828 \mathrm{E}-4$ \\
\hline 401.81 & 3.82 & -6.78 & 0.3381 E-4 \\
\hline 402.22 & 137.23 & -18.17 & $0.2363 \mathrm{E}-4$ \\
\hline 402.73 & -178.00 & 1.76 & $0.1924 \mathrm{E}-4$ \\
\hline 403.21 & 103.32 & -43.26 & 0.4757 E- 5 \\
\hline 403.72 & 75.32 & -4.21 & $0.1925 \mathrm{E}-4$ \\
\hline 404.24 & -78.95 & -13.28 & $0.2303 \mathrm{E}-4$ \\
\hline 404.76 & 46.58 & -4.72 & $0.2728 \mathrm{E}-4$ \\
\hline 405.24 & -127.99 & -11.63 & $0.1532 \mathrm{E}-4$ \\
\hline 405.74 & 134.91 & 22.31 & 0.1872 E-4 \\
\hline 406.26 & -173.93 & 11.89 & 0.1482 E-4 \\
\hline 406.74 & -54.19 & 13.15 & $0.2112 \mathrm{E}-4$ \\
\hline 407.24 & -78.33 & -1.88 & $0.2048 \mathrm{E}-4$ \\
\hline 407.59 & 97.09 & -0.89 & $0.2335 \mathrm{E}-4$ \\
\hline 407.93 & -23.41 & -0.92 & 0.4579 E- 5 \\
\hline 408.51 & -158.76 & 34.16 & 0.3397 E-5 \\
\hline 409.22 & -81.47 & 5.65 & 0.2485 E-5 \\
\hline 409.63 & 66.65 & 0.82 & 0.3848 E-4 \\
\hline 410.07 & 56.95 & -1.68 & 0.7768 E-5 \\
\hline 410.59 & 133.86 & -6.82 & $0.2093 \mathrm{E}-4$ \\
\hline 411.16 & 70.15 & -2.24 & 0.2894 E-4 \\
\hline 411.57 & -109.14 & -12.89 & 0.7080 E- 5 \\
\hline 412.09 & 15.58 & 24.43 & 0.2724 E- 5 \\
\hline 412.87 & 54.80 & -8.48 & 0.6336 E-5 \\
\hline 413.38 & 148.94 & -12.43 & 0.8179 E-5 \\
\hline 413.86 & -124.15 & -17.40 & $0.3058 \mathrm{E}-4$ \\
\hline 414.36 & 99.58 & 2.04 & 0.8275 E-5 \\
\hline 417.32 & 98.54 & -23.62 & 0.6353 E-5 \\
\hline 417.85 & 178.02 & -12.27 & 0.3399 E-5 \\
\hline 418.35 & -38.30 & 4.25 & 0.2667 E-4 \\
\hline 418.83 & -162.49 & -31.43 & $0.2654 \mathrm{E}-4$ \\
\hline 419.34 & 175.35 & 4.65 & $0.2616 \mathrm{E}-4$ \\
\hline 419.85 & -125.56 & -4.44 & $0.1622 \mathrm{E}-4$ \\
\hline 420.35 & 73.75 & -9.61 & 0.2971 E-4 \\
\hline 420.84 & -40.35 & 3.36 & $0.2615 \mathrm{E}-4$ \\
\hline 421.27 & 86.85 & -14.11 & 0.2953 E-4 \\
\hline 421.85 & 20.22 & -9.65 & 0.6591 E- 5 \\
\hline 422.34 & 80.44 & -7.40 & 0.2809 E-4 \\
\hline 422.83 & -12.24 & -2.45 & 0.1523 E-4 \\
\hline 423.38 & -72.92 & 8.32 & 0.2177 E-4 \\
\hline 427.76 & -45.64 & 1.60 & $0.1204 \mathrm{E}-4$ \\
\hline 429.08 & -10.27 & -33.85 & 0.1698 E-4 \\
\hline 429.55 & 50.94 & -40.31 & $0.2571 \mathrm{E}-5$ \\
\hline 430.13 & 176.79 & -26.51 & 0.8744 E-5 \\
\hline 430.58 & 137.05 & -7.67 & 0.9320 E- 5 \\
\hline 431.05 & 45.85 & -17.25 & $0.1393 \mathrm{E}-4$ \\
\hline 431.63 & 92.42 & -12.47 & $0.2017 \mathrm{E}-4$ \\
\hline 432.08 & 14.73 & -25.41 & $0.1083 \mathrm{E}-4$ \\
\hline 432.55 & 61.16 & -3.76 & $0.1575 \mathrm{E}-4$ \\
\hline 433.13 & -60.18 & -13.79 & $0.1636 \mathrm{E}-4$ \\
\hline 433.58 & 115.41 & -27.16 & $0.1550 \mathrm{E}-4$ \\
\hline 434.05 & 76.03 & -11.87 & 0.2365 E-4 \\
\hline 434.63 & 71.58 & -27.52 & 0.1131 E-4 \\
\hline 436.13 & -6.23 & -6.49 & $0.5424 \mathrm{E}-5$ \\
\hline 436.71 & 107.11 & 0.81 & 0.3451 E-4 \\
\hline 437.22 & 96.46 & -10.45 & 0.1157 E-4 \\
\hline 437.62 & 128.74 & -4.40 & 0.4012 E-4 \\
\hline
\end{tabular}

Appendix B. (Continued).

\begin{tabular}{|c|c|c|c|}
\hline $\begin{array}{l}\text { Sub-bottom } \\
\text { depth } \\
\text { (m) }\end{array}$ & $\begin{array}{l}\text { Declination } \\
\left(\left(^{\circ}\right)\right.\end{array}$ & $\begin{array}{l}\text { Inclination } \\
\left({ }^{\circ}\right)\end{array}$ & $\begin{array}{l}\text { Intensity } \\
(G)\end{array}$ \\
\hline \multicolumn{4}{|c|}{ Hole 572D (Cont.) } \\
\hline 438.51 & 46.73 & 0.51 & $0.5201 \mathrm{E}-4$ \\
\hline 438.78 & -78.05 & 2.87 & $0.3231 \mathrm{E}-4$ \\
\hline 439.27 & 83.08 & -6.39 & $0.6410 \mathrm{E}-4$ \\
\hline 439.83 & -141.86 & 5.24 & $0.3502 \mathrm{E}-4$ \\
\hline 441.02 & 45.37 & 13.30 & 0.4477 E-4 \\
\hline 441.61 & 53.81 & -1.41 & $0.1811 \mathrm{E}-4$ \\
\hline 442.20 & 61.70 & 0.51 & 0.3093 E-4 \\
\hline 443.18 & -171.07 & 17.29 & $0.2928 \mathrm{E}-4$ \\
\hline 443.81 & -126.31 & 12.38 & 0.4142 E-4 \\
\hline 445.63 & 30.23 & 13.44 & $0.4874 \mathrm{E}-4$ \\
\hline 446.32 & -71.31 & -36.34 & 0.7333 E-5 \\
\hline 446.69 & -11.51 & -23.65 & 0.7505 E-6 \\
\hline 447.31 & 23.53 & -35.48 & 0.9180 E-6 \\
\hline 448.49 & -118.56 & -2.09 & 0.4200 E-5 \\
\hline 450.41 & 14.08 & 0.98 & $0.3600 \mathrm{E}-4$ \\
\hline 451.02 & 76.11 & -5.69 & $0.3831 \mathrm{E}-4$ \\
\hline 451.38 & -14.94 & 2.83 & $0.5979 \mathrm{E}-4$ \\
\hline 451.61 & 136.38 & -18.41 & $0.4659 \mathrm{E}-4$ \\
\hline 452.01 & 163.55 & -2.47 & $0.4647 \mathrm{E}-4$ \\
\hline 452.55 & -60.05 & 5.24 & $0.4595 \mathrm{E}-4$ \\
\hline 452.94 & 48.25 & -6.44 & $0.7193 \mathrm{E}-4$ \\
\hline 453.15 & -85.56 & 1.03 & $0.4306 \mathrm{E}-4$ \\
\hline 453.64 & -130.66 & 3.68 & $0.3380 \mathrm{E}-4$ \\
\hline 455.31 & -63.25 & -0.78 & $0.5773 \mathrm{E}-4$ \\
\hline 455.71 & -50.25 & -0.61 & $0.5391 \mathrm{E}-4$ \\
\hline 456.18 & 166.31 & 1.99 & $0.3136 \mathrm{E}-4$ \\
\hline 456.61 & -50.23 & -11.23 & $0.2431 \mathrm{E}-4$ \\
\hline 457.31 & 6.88 & -17.91 & $0.2098 \mathrm{E}-4$ \\
\hline 457.56 & 66.30 & 0.24 & 0.3146 E-4 \\
\hline 458.73 & 17.09 & -18.95 & 0.2549 E-4 \\
\hline 459.38 & -69.94 & 12.30 & 0.5609 E-5 \\
\hline 459.91 & -106.51 & 21.59 & 0.2369 E-5 \\
\hline 461.00 & -21.26 & 21.40 & 0.1292 E-5 \\
\hline 461.91 & 95.69 & -3.38 & 0.1422 E-4 \\
\hline 462.33 & -162.62 & 0.72 & 0.1164 E-4 \\
\hline 463.36 & -63.33 & 1.89 & 0.3102 E-4 \\
\hline 463.96 & -71.69 & -1.50 & $0.4524 \mathrm{E}-4$ \\
\hline 464.16 & -95.37 & 3.56 & $0.3286 \mathrm{E}-4$ \\
\hline 464.41 & -80.51 & 3.40 & 0.8191 E-5 \\
\hline
\end{tabular}

Hole 573

\begin{tabular}{rrrr}
86.21 & 23.72 & -19.22 & 0.9955 E-6 \\
87.81 & 27.88 & -19.91 & 0.1026 E-5 \\
88.54 & 24.77 & -16.80 & 0.1072 E-5 \\
89.15 & 28.41 & -5.22 & 0.8568 E-6 \\
89.43 & 24.21 & -8.47 & 0.9709 E-6 \\
89.85 & 23.55 & -14.10 & 0.1104 E-5 \\
90.51 & 22.69 & -7.38 & 0.1111 E-5 \\
90.92 & 30.24 & -22.56 & 0.1188 E-5 \\
91.32 & 33.94 & -6.29 & 0.8573 E-6 \\
92.01 & 25.22 & -11.98 & 0.1053 E-5 \\
92.43 & 27.47 & -3.91 & 0.9334 E-6 \\
92.82 & 21.10 & -14.77 & 0.8963 E-6 \\
93.01 & 26.90 & -22.95 & 0.9816 E-6 \\
93.93 & 25.43 & -14.17 & 0.9546 E-6 \\
94.32 & 23.73 & -19.88 & 0.9472 E-6 \\
95.64 & 24.91 & -10.49 & 0.9491 E-6 \\
96.03 & 16.62 & -14.74 & 0.9864 E-6 \\
96.44 & 25.62 & -11.32 & 0.1093 E-5 \\
97.01 & 32.12 & -9.29 & 0.8938 E-6 \\
97.43 & 41.21 & -0.47 & 0.9953 E-6 \\
97.94 & 21.35 & -13.89 & 0.1012 E-5 \\
98.51 & 31.04 & -7.38 & 0.8489 E-6 \\
98.93 & 23.04 & -3.07 & 0.9959 E-6 \\
99.44 & 59.54 & -18.85 & 0.7823 E-6 \\
100.01 & 42.33 & 8.23 & 0.7683 E-6 \\
100.43 & 30.72 & -2.32 & 0.8443 E-6 \\
100.94 & 34.81 & -9.78 & 0.8179 E-6 \\
101.51 & 23.96 & -21.32 & 0.1053 E-5 \\
101.93 & 44.61 & -5.53 & 0.1017 E-5 \\
102.44 & 43.06 & -41.63 & 0.8287 E-6 \\
103.01 & 25.43 & -13.11 & 0.9394 E-6 \\
103.43 & 35.21 & -23.91 & 0.8869 E-6 \\
\hline & & & \\
\hline 93 & & &
\end{tabular}


Appendix B. (Continued).

\begin{tabular}{cccc}
\hline $\begin{array}{c}\text { Sub-bottom } \\
\text { depth } \\
(\mathrm{m})\end{array}$ & $\begin{array}{c}\text { Declination } \\
\left({ }^{\circ}\right)\end{array}$ & $\begin{array}{c}\text { Inclination } \\
\left({ }^{\circ}\right)\end{array}$ & $\begin{array}{c}\text { Intensity } \\
(\mathrm{G})\end{array}$ \\
\hline
\end{tabular}

\section{Hole 573 (Cont.)}

\begin{tabular}{|c|c|c|c|}
\hline 103.94 & 35.85 & 9.47 & $0.9653 \mathrm{E}-6$ \\
\hline 105.40 & 25.45 & 0.96 & 0.9804 E-6 \\
\hline 105.82 & 31.99 & 6.00 & 0.8664 E-6 \\
\hline 106.22 & 27.52 & -17.34 & 0.9700 E-6 \\
\hline 106.63 & 31.45 & 12.87 & 0.9875 E-6 \\
\hline 107.32 & 31.51 & -0.87 & 0.9585 E-6 \\
\hline 107.72 & 13.01 & -4.14 & 0.8362 E-6 \\
\hline 108.13 & 26.22 & 3.83 & 0.9893 E-6 \\
\hline 108.82 & 76.76 & -9.58 & 0.1185 E-5 \\
\hline 109.22 & 39.59 & 13.03 & 0.7803 E-6 \\
\hline 109.63 & 19.69 & 2.05 & 0.1078 E-5 \\
\hline 110.31 & 26.58 & 2.99 & 0.9746 E-6 \\
\hline 110.72 & 22.80 & 2.98 & 0.9987 E-6 \\
\hline 111.82 & 20.95 & -11.44 & 0.8673 E-6 \\
\hline 112.22 & 24.11 & 2.60 & $0.9206 \mathrm{E}-6$ \\
\hline 112.63 & 30.47 & -3.26 & 0.8608 E- 6 \\
\hline 113.04 & 49.52 & 10.16 & 0.1032 E- 5 \\
\hline 113.45 & 27.40 & 3.48 & 0.7606 E- 6 \\
\hline 113.87 & 38.93 & 34.22 & 0.9630 E- 6 \\
\hline 114.54 & 21.83 & 9.72 & 0.9329 E-6 \\
\hline 114.95 & 24.04 & -6.69 & 0.9261 E-6 \\
\hline 115.37 & 27.16 & 30.72 & 0.1442 E-5 \\
\hline 116.04 & 2.25 & -18.19 & 0.4428 E- 6 \\
\hline 116.45 & 31.44 & 12.46 & 0.9073 E-6 \\
\hline 116.87 & 37.53 & -14.76 & 0.7448 E- 6 \\
\hline 117.54 & 32.80 & 20.03 & 0.8411 E-6 \\
\hline 117.95 & 29.00 & 5.21 & 0.1104 E-5 \\
\hline 118.37 & 32.30 & 6.29 & 0.9178 E-6 \\
\hline 119.04 & 45.01 & 1.69 & 0.2398 E-5 \\
\hline 119.45 & 24.29 & 7.48 & 0.9006 E- 6 \\
\hline 119.87 & 28.06 & 1.50 & 0.9696 E-6 \\
\hline 120.54 & 21.75 & 5.83 & 0.1006 E-5 \\
\hline 120.95 & 51.48 & -2.96 & 0.1089 E-5 \\
\hline 121.37 & 33.07 & 0.96 & 0.8907 E- 6 \\
\hline 121.72 & 34.28 & 2.15 & 0.9283 E-6 \\
\hline 122.41 & 22.64 & 5.30 & 0.9404 E-6 \\
\hline 122.82 & 61.89 & 37.45 & 0.7244 E- 6 \\
\hline 123.22 & 44.85 & 33.44 & 0.6481 E- 6 \\
\hline 123.91 & -46.36 & -33.53 & 0.1648 E-5 \\
\hline 124.32 & 40.05 & -6.55 & 0.8978 E-6 \\
\hline 124.72 & 69.17 & 1.57 & 0.3192 E- 5 \\
\hline 125.41 & 20.61 & -26.06 & 0.8318 E- 6 \\
\hline 125.82 & 26.05 & 8.35 & 0.1318 E-5 \\
\hline 126.22 & 31.07 & 26.91 & 0.1052 E-5 \\
\hline 126.91 & 42.66 & 27.79 & 0.1101 E-5 \\
\hline 127.32 & 29.47 & 7.04 & 0.1297 E-5 \\
\hline 127.72 & 18.92 & -20.03 & 0.1878 E-5 \\
\hline 128.41 & 33.21 & 3.78 & 0.1601 E-5 \\
\hline 128.82 & 26.15 & 13.52 & 0.1567 E-5 \\
\hline 129.22 & 10.43 & 14.17 & 0.1365 E-5 \\
\hline 129.91 & 9.68 & -7.14 & 0.1353 E-5 \\
\hline 130.32 & 19.98 & 14.18 & 0.1084 E-5 \\
\hline 130.72 & 16.39 & 11.46 & 0.1040 E-5 \\
\hline 131.61 & 39.01 & 24.43 & 0.1875 E-5 \\
\hline 132.01 & 17.06 & -11.45 & 0.3362 E- 5 \\
\hline 132.53 & 7.31 & 5.15 & 0.1368 E-5 \\
\hline 133.52 & 15.21 & 8.16 & 0.1292 E-5 \\
\hline 135.02 & 6.37 & 4.74 & 0.1430 E-5 \\
\hline 136.52 & 14.48 & 14.71 & 0.1188 E-5 \\
\hline 138.02 & 5.75 & -2.56 & 0.1616 E-5 \\
\hline 139.52 & 11.62 & -7.76 & 0.1586 E-5 \\
\hline 140.81 & 19.50 & 4.25 & 0.7739 E-6 \\
\hline 142.38 & 7.54 & 1.80 & 0.1501 E-5 \\
\hline 143.87 & 9.36 & 1.80 & 0.1467 E-5 \\
\hline 145.37 & 3.58 & 7.59 & 0.1055 E-5 \\
\hline 146.81 & 8.17 & 8.97 & 0.1459 E-5 \\
\hline 148.37 & 12.30 & 14.41 & 0.1075 E-5 \\
\hline 150.69 & 5.30 & 8.20 & 0.1670 E-5 \\
\hline 152.11 & 10.16 & 3.56 & 0.1565 E-5 \\
\hline 153.61 & 12.11 & 5.64 & 0.1432 E- 5 \\
\hline 154.36 & 5.80 & -18.86 & 0.1494 E-5 \\
\hline 155.86 & 7.10 & -6.06 & 0.1502 E-5 \\
\hline 157.77 & 20.82 & -12.47 & 0.1587 E-5 \\
\hline 158.84 & 14.42 & -13.32 & 0.1059 E-5 \\
\hline
\end{tabular}

Appendix B. (Continued).

\begin{tabular}{cccc}
\hline $\begin{array}{c}\text { Sub-bottom } \\
\text { depth } \\
(\mathrm{m})\end{array}$ & $\begin{array}{c}\text { Declination } \\
\left({ }^{\circ}\right)\end{array}$ & $\begin{array}{c}\text { Inclination } \\
\left({ }^{\circ}\right)\end{array}$ & $\begin{array}{c}\text { Intensity } \\
(\mathrm{G})\end{array}$
\end{tabular}

\section{Hole 573B}

\begin{tabular}{|c|c|c|c|}
\hline 142.36 & -3.48 & 16.36 & 0.7763 E-6 \\
\hline 143.97 & -27.05 & 33.28 & 0.6505 E-6 \\
\hline 144.97 & -2.55 & 19.79 & 0.5568 E-6 \\
\hline 152.29 & -13.15 & -14.26 & 0.7916 E-6 \\
\hline 155.27 & 12.83 & -23.12 & 0.5982 E-6 \\
\hline 158.74 & -9.33 & -13.35 & 0.1085 E-5 \\
\hline 164.77 & -7.16 & -5.92 & 0.8191 E-6 \\
\hline 176.03 & -16.11 & -7.67 & 0.9562 E-6 \\
\hline 194.51 & 6.41 & 5.01 & 0.2402 E-5 \\
\hline 195.49 & -13.09 & 1.49 & 0.1063 E-5 \\
\hline 195.83 & -10.46 & -16.54 & 0.9053 E-6 \\
\hline 208.21 & -5.61 & -10.90 & 0.8185 E-6 \\
\hline 211.58 & -15.07 & -13.18 & 0.1155 E-5 \\
\hline 218.47 & -9.28 & -14.07 & 0.9271 E-6 \\
\hline 222.52 & -5.57 & -12.21 & 0.8315 E-6 \\
\hline 226.47 & -9.48 & 2.97 & 0.8793 E-6 \\
\hline 229.48 & 17.52 & -15.09 & 0.1660 E-5 \\
\hline 232.34 & -7.49 & -9.08 & 0.1147 E-5 \\
\hline 235.39 & -9.14 & -4.67 & 0.1034 E-5 \\
\hline 238.39 & 75.72 & -41.19 & 0.6343 E-6 \\
\hline 245.23 & -0.67 & 5.27 & 0.9579 E-6 \\
\hline 247.91 & -13.44 & -6.62 & 0.9069 E-6 \\
\hline 254.47 & -2.46 & -8.96 & 0.8465 E- 6 \\
\hline 257.27 & -43.96 & -3.59 & 0.3488 E-6 \\
\hline 262.71 & 126.90 & -67.92 & 0.3921 E-5 \\
\hline 263.18 & 152.60 & 1.97 & 0.8968 E-5 \\
\hline 263.85 & 158.01 & 12.83 & $0.1067 \mathrm{E}-4$ \\
\hline 264.34 & 10.55 & 10.97 & 0.3819 E-5 \\
\hline 264.84 & -92.07 & -9.79 & 0.3357 E-5 \\
\hline 265.83 & 12.11 & 4.09 & $0.1434 \mathrm{E}-4$ \\
\hline 265.85 & -113.28 & -1.23 & 0.8724 E-5 \\
\hline 266.34 & -81.93 & 2.62 & 0.7963 E-6 \\
\hline 266.79 & -66.41 & 20.68 & $0.1091 \mathrm{E}-4$ \\
\hline 267.39 & 22.03 & -19.93 & 0.2294 E-5 \\
\hline 267.92 & -133.81 & 12.03 & 0.6803 E-5 \\
\hline 268.19 & -24.30 & 3.25 & 0.3354 E-5 \\
\hline 268.86 & 31.36 & -2.32 & 0.1702 E-5 \\
\hline 269.33 & -91.14 & -35.33 & 0.1746 E-5 \\
\hline 269.79 & 44.14 & 18.83 & 0.2735 E-5 \\
\hline 270.30 & 38.93 & 5.04 & 0.8556 E-5 \\
\hline 270.88 & -93.38 & -9.58 & 0.8369 E-5 \\
\hline 271.31 & -51.93 & 4.44 & 0.2675 E-5 \\
\hline 271.75 & -68.92 & 2.29 & 0.6279 E-5 \\
\hline 272.47 & 73.34 & -0.71 & 0.3252 E-5 \\
\hline 272.65 & 29.95 & 54.79 & $0.1649 \mathrm{E}-3$ \\
\hline 273.30 & 1.27 & -14.26 & 0.2003 E-5 \\
\hline 273.85 & 61.17 & -21.78 & 0.1701 E-5 \\
\hline 274.43 & 14.31 & 21.77 & 0.1156 E-5 \\
\hline 274.84 & 109.63 & 1.37 & 0.2742 E-5 \\
\hline 275.49 & -158.77 & 17.87 & 0.9944 E-6 \\
\hline 276.70 & 24.78 & -0.03 & 0.2334 E-5 \\
\hline 277.03 & -134.29 & -15.78 & 0.3077 E-5 \\
\hline 277.77 & 169.35 & -17.68 & 0.6023 E-5 \\
\hline 278.65 & 36.79 & -9.31 & 0.1965 E-5 \\
\hline 279.69 & -75.92 & 0.74 & 0.3634 E-5 \\
\hline 281.06 & -71.61 & 11.08 & 0.2286 E-5 \\
\hline 281.73 & 103.88 & -4.28 & 0.5347 E-5 \\
\hline 282.21 & 35.24 & -2.86 & 0.7671 E-5 \\
\hline 282.82 & -87.06 & -5.26 & $0.5426 \mathrm{E}-5$ \\
\hline 283.32 & -40.73 & -8.96 & 0.3548 E-5 \\
\hline 283.92 & 36.47 & -5.45 & 0.2603 E-5 \\
\hline 284.54 & -27.46 & -5.29 & 0.1903 E-5 \\
\hline 285.27 & 37.00 & -27.47 & 0.9356 E-6 \\
\hline 286.17 & -54.40 & 34.91 & 0.1466 E-5 \\
\hline 286.62 & -114.84 & -2.39 & 0.5767 E-5 \\
\hline 287.13 & 175.08 & -1.11 & 0.4377 E-5 \\
\hline 287.57 & 172.02 & 4.80 & 0.9397 E-5 \\
\hline 288.40 & 23.10 & 10.87 & 0.4489 E-5 \\
\hline 290.63 & -125.40 & -36.20 & 0.1221 E-5 \\
\hline 291.10 & -34.57 & -12.26 & 0.2990 E-5 \\
\hline 291.67 & 37.29 & -7.48 & 0.1681 E-5 \\
\hline 292.09 & -90.20 & 37.44 & 0.1097 E-5 \\
\hline 292.89 & 159.39 & 16.11 & 0.2984 E-5 \\
\hline 293.12 & -9.91 & 1.68 & 0.4774 E-5 \\
\hline
\end{tabular}


Appendix B. (Continued).

\begin{tabular}{cccc}
\hline $\begin{array}{c}\text { Sub-bottom } \\
\text { depth } \\
(\mathrm{m})\end{array}$ & $\begin{array}{c}\text { Declination } \\
\left({ }^{\circ}\right)\end{array}$ & $\begin{array}{c}\text { Inclination } \\
\left({ }^{\circ}\right)\end{array}$ & $\begin{array}{c}\text { Intensity } \\
(\mathrm{G})\end{array}$ \\
\hline
\end{tabular}

\section{Hole 573B (Cont.)}

\begin{tabular}{|c|c|c|c|}
\hline 293.69 & 162.76 & 15.20 & $0.3546 \mathrm{E}$ \\
\hline 296.11 & -131.53 & 2.90 & $0.1730 \mathrm{E}$ \\
\hline 300.35 & -73.17 & 26.28 & $0.7788 \mathrm{E}$ \\
\hline 301.70 & 169.34 & 1.56 & $0.2245 \mathrm{E}$ \\
\hline 302.44 & -110.61 & -6.75 & $0.3257 \mathrm{E}$ \\
\hline 303.05 & 1.45 & -13.76 & $0.1619 \mathrm{E}$ \\
\hline 303.62 & -54.66 & 3.74 & $0.2483 \mathrm{E}$ \\
\hline 304.06 & -81.02 & -4.04 & $0.2845 \mathrm{E}$ \\
\hline 309.66 & 91.88 & 24.74 & $0.1601 \mathrm{E}$ \\
\hline 310.13 & 85.09 & 41.56 & $0.2700 \mathrm{E}$ \\
\hline 312.34 & -25.76 & -20.87 & $0.1287 \mathrm{E}$ \\
\hline 312.65 & -26.14 & 6.13 & $0.3160 \mathrm{E}$ \\
\hline 319.31 & 72.29 & -5.06 & $0.3639 \mathrm{E}$ \\
\hline 319.75 & -15.43 & 11.99 & $0.1200 \mathrm{E}$ \\
\hline 320.21 & -107.50 & -20.09 & $0.3259 \mathrm{E}$ \\
\hline 320.73 & -154.99 & 14.37 & $0.1337 \mathrm{E}$ \\
\hline 321.18 & 178.93 & 7.22 & $0.9509 \mathrm{E}$ \\
\hline 321.67 & -40.87 & 6.66 & $0.1291 \mathrm{E}$ \\
\hline 322.28 & -149.77 & -4.70 & $0.1373 \mathrm{E}$ \\
\hline 322.61 & 88.39 & -10.42 & $0.6825 \mathrm{E}$ \\
\hline 324.73 & -110.58 & -7.28 & $0.1464 \mathrm{E}$ \\
\hline 325.19 & 147.45 & -10.71 & $0.9675 \mathrm{E}$ \\
\hline 325.93 & -121.45 & 18.05 & $0.2280 \mathrm{E}$ \\
\hline 326.76 & 159.89 & 24.15 & $0.1566 \mathrm{E}$ \\
\hline 327.83 & 26.56 & 12.56 & $0.8943 \mathrm{E}$ \\
\hline 328.10 & -147.20 & 6.68 & $0.9576 \mathrm{E}$ \\
\hline 328.62 & 14.25 & 4.48 & $0.1614 \mathrm{E}$ \\
\hline 329.52 & 50.84 & -3.84 & $0.2894 \mathrm{E}$ \\
\hline 329.95 & -61.68 & 2.59 & $0.2324 \mathrm{E}$ \\
\hline 330.05 & 128.47 & -3.28 & $0.5178 \mathrm{E}$ \\
\hline 331.74 & 40.22 & 2.22 & $0.5733 \mathrm{E}$ \\
\hline 332.64 & -125.41 & -29.77 & $0.1772 \mathrm{E}$ \\
\hline 338.06 & -70.58 & -10.66 & $0.4178 \mathrm{E}$ \\
\hline 338.58 & -51.32 & -11.75 & $0.8574 \mathrm{E}$ \\
\hline 339.56 & -134.77 & -10.71 & $0.1148 \mathrm{E}$ \\
\hline 340.58 & -160.80 & -9.96 & $0.6950 \mathrm{E}$ \\
\hline 342.61 & -101.35 & 3.46 & $0.1970 \mathrm{E}$ \\
\hline 343.11 & -98.33 & -5.13 & $0.9847 \mathrm{E}$ \\
\hline 348.75 & 104.53 & 1.69 & $0.6326 \mathrm{E}$ \\
\hline 350.81 & -146.67 & -23.11 & $0.6124 \mathrm{E}$ \\
\hline 352.54 & -171.57 & -38.14 & $0.2393 \mathrm{E}$ \\
\hline 357.23 & 35.25 & -55.40 & $0.3830 \mathrm{E}$ \\
\hline 357.81 & -133.69 & -15.61 & $0.1155 \mathrm{E}$ \\
\hline 358.86 & -116.82 & -29.49 & $0.5531 \mathrm{E}$ \\
\hline 359.87 & 8.59 & -68.75 & $0.1017 \mathrm{E}$ \\
\hline 360.29 & -145.86 & -28.99 & $0.2135 \mathrm{E}$ \\
\hline 360.64 & -46.90 & -1.57 & $0.7405 \mathrm{E}$ \\
\hline 361.36 & -53.66 & -12.91 & $0.2598 \mathrm{E}$ \\
\hline 361.54 & 74.01 & -28.51 & $0.1542 \mathrm{E}$ \\
\hline 366.69 & -94.48 & -17.81 & $0.2459 \mathrm{E}$ \\
\hline 367.29 & 104.72 & 2.06 & $0.3896 \mathrm{E}$ \\
\hline 367.65 & 6.12 & -45.35 & $0.7391 \mathrm{E}$ \\
\hline 377.66 & -127.01 & 23.13 & $0.8867 \mathrm{E}$ \\
\hline 378.06 & -122.70 & 1.47 & $0.3539 \mathrm{E}$ \\
\hline 378.55 & -36.81 & 9.35 & $0.8119 \mathrm{E}$ \\
\hline 379.12 & 2.85 & -1.35 & $0.1317 \mathrm{E}$ \\
\hline 379.88 & -15.36 & -14.50 & $0.9022 \mathrm{E}$ \\
\hline 380.21 & -171.80 & 19.88 & $0.7166 \mathrm{E}$ \\
\hline 380.64 & 56.45 & -17.48 & $0.5820 \mathrm{E}$ \\
\hline 381.50 & 12.81 & -43.76 & $0.2222 \mathrm{E}$ \\
\hline 382.23 & 135.31 & -15.02 & $0.4045 \mathrm{E}$ \\
\hline 383.05 & 5.73 & -23.06 & $0.8870 \mathrm{E}$ \\
\hline 385.66 & -98.72 & -17.65 & $0.5337 \mathrm{E}$ \\
\hline 386.25 & -127.45 & -20.58 & $0.6318 \mathrm{E}$ \\
\hline 386.88 & -7.57 & -18.02 & $0.1104 \mathrm{E}$ \\
\hline 387.53 & 129.43 & 9.31 & $0.4779 \mathrm{E}$ \\
\hline 388.03 & -6.74 & -12.52 & $0.1541 \mathrm{E}$ \\
\hline 388.57 & -82.63 & -24.86 & $0.9358 \mathrm{E}$ \\
\hline 389.07 & -20.34 & -16.57 & $0.4100 \mathrm{E}$ \\
\hline 389.77 & 22.88 & -23.60 & $0.1440 \mathrm{E}$ \\
\hline 390.47 & -153.11 & -15.11 & $0.5431 \mathrm{E}$ \\
\hline 390.98 & 14.63 & -2.53 & $0.1817 \mathrm{E}$ \\
\hline
\end{tabular}

Appendix B. (Continued).

\begin{tabular}{cccc}
$\begin{array}{c}\text { Sub-bottom } \\
\text { depth } \\
(\mathrm{m})\end{array}$ & $\begin{array}{c}\text { Declination } \\
\left({ }^{\circ}\right)\end{array}$ & $\begin{array}{c}\text { Inclination } \\
\left({ }^{\circ}\right)\end{array}$ & $\begin{array}{c}\text { Intensity } \\
(\mathrm{G})\end{array}$ \\
\hline
\end{tabular}

Hole 573B (Cont.)

\begin{tabular}{|c|c|c|c|}
\hline 391.46 & 71.43 & 14.77 & 0.1064 E-5 \\
\hline 391.92 & -35.39 & -3.71 & 0.7785 E-6 \\
\hline 396.34 & -26.35 & -17.69 & 0.3195 E-5 \\
\hline 396.99 & 0.36 & -12.71 & 0.1076 E-5 \\
\hline 398.75 & -19.46 & 3.32 & 0.5444 E-6 \\
\hline 399.24 & 12.90 & 18.20 & 0.2169 E-5 \\
\hline 404.57 & 102.67 & 10.02 & 0.3522 E- 5 \\
\hline 404.98 & -121.21 & 30.19 & 0.6779 E-6 \\
\hline 405.73 & 105.01 & -59.02 & 0.6213 E-6 \\
\hline 406.29 & 8.36 & -13.91 & 0.2309 E-5 \\
\hline 406.86 & 13.34 & 2.93 & 0.1580 E-5 \\
\hline 407.43 & -108.95 & 13.77 & 0.6658 E-6 \\
\hline 408.36 & 12.57 & -10.04 & 0.6624 E-6 \\
\hline 408.61 & 2.04 & -6.00 & 0.3624 E-6 \\
\hline 409.19 & -20.50 & -34.89 & 0.5770 E-6 \\
\hline 410.01 & -11.80 & -5.94 & 0.5892 E-6 \\
\hline 410.91 & 16.57 & -21.89 & 0.6050 E-6 \\
\hline 411.23 & 17.62 & -14.97 & 0.7586 E-6 \\
\hline 414.33 & -14.49 & -31.00 & 0.6793 E-6 \\
\hline 415.25 & 4.45 & -33.34 & 0.4999 E-6 \\
\hline 418.28 & -32.64 & 1.81 & 0.4464 E-6 \\
\hline 418.79 & 11.05 & -35.19 & 0.5381 E-6 \\
\hline 424.07 & 175.16 & 23.15 & 0.1697 E-5 \\
\hline 424.64 & 71.38 & -37.83 & $0.3329 \mathrm{E}-6$ \\
\hline 425.45 & -146.19 & -20.66 & $0.5830 \mathrm{E}-6$ \\
\hline 433.25 & -29.70 & -32.21 & 0.5071 E-6 \\
\hline 434.30 & 2.04 & -49.06 & 0.4829 E-6 \\
\hline 435.07 & -21.80 & -15.24 & 0.1427 E-5 \\
\hline 435.48 & -2.84 & -13.51 & 0.9517 E-6 \\
\hline 435.80 & -23.32 & -21.05 & 0.4468 E- 6 \\
\hline 436.06 & 5.14 & -37.47 & 0.6220 E-6 \\
\hline 436.57 & 13.63 & -23.41 & 0.3267 E-6 \\
\hline 442.74 & 52.58 & -10.44 & 0.7661 E-6 \\
\hline 447.69 & -65.33 & 8.87 & $0.6548 \mathrm{E}-6$ \\
\hline 452.15 & 24.65 & -21.61 & $0.6318 \mathrm{E}-6$ \\
\hline 452.55 & 66.18 & -12.20 & 0.8267 E-6 \\
\hline 453.36 & 39.78 & 13.17 & 0.2101 E-5 \\
\hline 453.63 & -29.45 & 12.52 & 0.1961 E-5 \\
\hline 453.97 & 22.36 & -12.31 & 0.2094 E-5 \\
\hline 461.69 & -32.23 & -27.03 & 0.8095 E-6 \\
\hline 462.51 & -4.80 & -2.12 & 0.4820 E-6 \\
\hline 463.53 & -29.01 & -1.70 & 0.6072 E-6 \\
\hline 464.96 & -27.17 & -61.94 & $0.8356 \mathrm{E}-6$ \\
\hline 465.59 & -3.83 & -47.50 & 0.1450 E- 5 \\
\hline 466.12 & -14.14 & -48.15 & 0.9443 E-6 \\
\hline 466.51 & 5.19 & -39.94 & 0.1039 E-5 \\
\hline 467.01 & 1.05 & -36.00 & 0.6648 E-6 \\
\hline 467.61 & -18.21 & -31.69 & 0.9446 E-6 \\
\hline 468.43 & -31.19 & 34.48 & 0.8813 E-6 \\
\hline 471.27 & -34.38 & 19.41 & 0.9195 E-6 \\
\hline 471.70 & -5.71 & 28.11 & 0.9408 E-6 \\
\hline 472.30 & -11.41 & -36.00 & 0.1291 E- 5 \\
\hline 473.96 & -19.03 & -37.41 & 0.1375 E- 5 \\
\hline 474.03 & -35.68 & -39.57 & 0.1206 E-5 \\
\hline 480.62 & -23.28 & 1.75 & 0.4327 E-6 \\
\hline 490.14 & -161.24 & 7.57 & 0.1907 E-5 \\
\hline 492.43 & -28.98 & 4.98 & 0.7220 E-6 \\
\hline 494.02 & -21.97 & -8.18 & 0.7251 E-6 \\
\hline 499.77 & -152.15 & -15.96 & 0.5986 E-5 \\
\hline 501.14 & -108.37 & -3.21 & 0.1513 E-5 \\
\hline 502.02 & -32.39 & 8.87 & 0.9539 E-5 \\
\hline 502.85 & -39.21 & 15.21 & $0.2282 \mathrm{E}-5$ \\
\hline 503.37 & -79.10 & 11.46 & $0.1810 \mathrm{E}-4$ \\
\hline 503.58 & -102.37 & 14.02 & $0.1488 \mathrm{E}-4$ \\
\hline 510.24 & 61.41 & 14.09 & 0.2007 E-4 \\
\hline 520.63 & -110.70 & 1.02 & 0.1584 E-4 \\
\hline 521.07 & -112.64 & 2.11 & 0.1954 E-4 \\
\hline 521.47 & -112.67 & -1.46 & $0.1341 \mathrm{E}-4$ \\
\hline 521.91 & -111.50 & 4.88 & 0.1761 E-4 \\
\hline 522.37 & -40.59 & 6.42 & $0.2150 \mathrm{E}-4$ \\
\hline 523.65 & -89.88 & -0.25 & 0.8600 E-4 \\
\hline 526.06 & -114.01 & -13.11 & 0.3485 E-3 \\
\hline
\end{tabular}


Appendix B. (Continued).

\begin{tabular}{|c|c|c|c|}
\hline $\begin{array}{l}\text { Sub-bottom } \\
\text { depth } \\
\text { (m) }\end{array}$ & $\begin{array}{c}\text { Declination } \\
\left({ }^{\circ}\right)\end{array}$ & $\begin{array}{c}\text { Inclination } \\
\left({ }^{\circ}\right)\end{array}$ & $\begin{array}{l}\text { Intensity } \\
\text { (G) }\end{array}$ \\
\hline \multicolumn{4}{|c|}{ Hole 573B (Cont.) } \\
\hline 526.35 & -139.06 & -47.52 & $0.6530 \mathrm{E}-4$ \\
\hline 526.55 & 179.56 & 10.27 & $0.2383 \mathrm{E}-4$ \\
\hline 527.00 & -128.69 & -1.69 & $0.1553 \mathrm{E}-4$ \\
\hline \multicolumn{4}{|l|}{ Hole 574} \\
\hline 93.34 & 15.74 & 5.72 & 0.1262 E-5 \\
\hline 96.39 & 16.33 & 10.50 & 0.1451 E-5 \\
\hline 100.22 & 8.63 & 3.83 & $0.1533 \mathrm{E}-5$ \\
\hline 101.74 & 6.54 & -1.58 & 0.1547 E-5 \\
\hline 103.79 & 10.15 & -7.07 & 0.1410 E-5 \\
\hline 110.69 & 11.50 & 4.71 & 0.1534 E-5 \\
\hline 113.86 & 8.98 & -13.74 & 0.8777 E-6 \\
\hline 115.96 & 10.66 & 2.42 & 0.1459 E-5 \\
\hline 117.16 & 11.24 & 1.57 & $0.1482 \mathrm{E}-5$ \\
\hline 121.02 & 7.91 & 4.56 & 0.1419 E-5 \\
\hline 127.71 & 12.05 & 11.96 & $0.1402 \mathrm{E}-5$ \\
\hline 127.96 & 9.52 & 11.38 & 0.1352 E- 5 \\
\hline 128.26 & -5.33 & 8.55 & $0.1327 \mathrm{E}-5$ \\
\hline 128.48 & 9.91 & -2.16 & 0.1145 E-5 \\
\hline 128.91 & 11.32 & 0.52 & $0.1216 \mathrm{E}-5$ \\
\hline 129.40 & -12.44 & 6.81 & $0.1391 \mathrm{E}-5$ \\
\hline 129.98 & 75.68 & 0.99 & 0.5927 E-6 \\
\hline 130.40 & 51.12 & -13.05 & 0.4902 E- 6 \\
\hline 130.90 & 10.50 & 1.62 & $0.1269 \mathrm{E}-5$ \\
\hline 131.48 & 10.91 & 13.06 & $0.1313 \mathrm{E}-5$ \\
\hline 131.90 & 43.68 & -7.80 & 0.3600 E-6 \\
\hline 133.54 & 52.54 & -41.28 & 0.8282 E-6 \\
\hline 135.04 & 20.80 & 6.69 & 0.1650 E-5 \\
\hline 136.54 & 18.67 & -16.38 & 0.1537 E-5 \\
\hline 138.54 & 20.72 & 2.04 & 0.1580 E-5 \\
\hline 140.04 & 28.60 & -1.38 & 0.1246 E-5 \\
\hline 141.54 & 21.22 & -1.05 & 0.1569 E-5 \\
\hline 145.01 & 26.74 & -8.48 & 0.1283 E-6 \\
\hline 146.34 & 30.94 & -2.09 & 0.1590 E-5 \\
\hline 148.91 & 21.38 & -5.81 & 0.1716 E-5 \\
\hline 150.41 & 21.95 & -3.68 & 0.1374 E-5 \\
\hline 151.91 & 33.03 & 7.32 & 0.1461 E- 5 \\
\hline 153.81 & 52.13 & -6.86 & 0.1003 E-5 \\
\hline 155.31 & 26.84 & -8.26 & 0.1368 E-5 \\
\hline 156.81 & 30.68 & 8.71 & 0.1570 E-5 \\
\hline 158.81 & 54.74 & -37.27 & 0.1014 E-5 \\
\hline 160.31 & 55.51 & -35.46 & 0.9009 E-6 \\
\hline 161.81 & 61.59 & -42.07 & 0.7480 E-6 \\
\hline 163.81 & 50.98 & -27.94 & 0.8071 E-6 \\
\hline 165.31 & 78.09 & -27.78 & 0.6284 E-6 \\
\hline 166.66 & 60.62 & -38.43 & 0.8329 E-6 \\
\hline 166.81 & 69.09 & -39.44 & 0.8563 E-6 \\
\hline 168.81 & 59.61 & -28.17 & 0.7275 E-6 \\
\hline 170.31 & 66.36 & -40.39 & 0.8703 E-6 \\
\hline 171.81 & 79.49 & -35.24 & 0.7439 E-6 \\
\hline 173.35 & 47.42 & -28.84 & 0.1023 E-5 \\
\hline 175.16 & 65.70 & -35.96 & 0.8586 E-6 \\
\hline 179.88 & 44.65 & -41.10 & 0.8922 E-6 \\
\hline 183.24 & 48.12 & -41.15 & 0.8318 E-6 \\
\hline 183.77 & 49.38 & -36.07 & 0.9404 E-6 \\
\hline 184.63 & -116.99 & 17.83 & 0.2612 E-5 \\
\hline 185.66 & -16.54 & -2.83 & 0.9616 E-6 \\
\hline 187.35 & -43.85 & -28.84 & $0.1156 \mathrm{E}-5$ \\
\hline 188.85 & -34.53 & -23.40 & 0.8712 E-6 \\
\hline 193.74 & 7.48 & 7.14 & 0.1429 E-5 \\
\hline 196.83 & 15.04 & -10.59 & 0.8767 E-6 \\
\hline 197.02 & 119.67 & -41.13 & $0.4188 \mathrm{E}-6$ \\
\hline 199.64 & -37.39 & -13.28 & 0.2298 E-5 \\
\hline 199.85 & 51.17 & -8.44 & 0.2696 E-5 \\
\hline 200.05 & 49.67 & -8.47 & 0.2832 E-5 \\
\hline 200.32 & 46.45 & 9.63 & 0.1254 E-5 \\
\hline 200.50 & 87.35 & -0.38 & $0.4257 \mathrm{E}-4$ \\
\hline 200.86 & 38.04 & 7.48 & 0.1288 E-5 \\
\hline 201.16 & 52.76 & 17.87 & 0.1883 E-5 \\
\hline 201.36 & -58.98 & -13.46 & 0.3352 E-5 \\
\hline 201.56 & -97.02 & -0.05 & $0.1598 \mathrm{E}-4$ \\
\hline 201.77 & -110.41 & -3.22 & $0.2250 \mathrm{E}-4$ \\
\hline 201.98 & -71.31 & 19.98 & $0.1186 \mathrm{E}-4$ \\
\hline
\end{tabular}

Appendix B. (Continued).

\begin{tabular}{ccrc}
\hline $\begin{array}{c}\text { Sub-bottom } \\
\text { depth } \\
(\mathrm{m})\end{array}$ & $\begin{array}{c}\text { Declination } \\
\left({ }^{\circ}\right)\end{array}$ & $\begin{array}{c}\text { Inclination } \\
\left({ }^{\circ}\right)\end{array}$ & $\begin{array}{c}\text { Intensity } \\
(\mathrm{G})\end{array}$ \\
\hline Hole 574 (Cont.) & & & \\
& & & \\
202.53 & 128.58 & -24.90 & 0.6949 E-5 \\
202.76 & 137.30 & 9.33 & 0.1053 E-4 \\
203.06 & 122.50 & -11.86 & 0.6840 E-5 \\
203.36 & 117.13 & 10.45 & 0.5108 E-5 \\
203.66 & 79.40 & -11.10 & 0.3030 E-5 \\
204.03 & 97.01 & -15.92 & 0.1374 E-4 \\
204.26 & 87.75 & -7.32 & 0.9311 E-5 \\
204.56 & 78.33 & 15.84 & 0.1846 E-4 \\
205.16 & 41.16 & 9.38 & 0.2323 E-5 \\
205.53 & 4.90 & -14.65 & 0.1995 E-5 \\
205.76 & 10.50 & -41.27 & 0.2302 E-5 \\
206.06 & 15.46 & -53.03 & 0.2849 E-5 \\
206.36 & 31.17 & -55.06 & 0.6456 E-5 \\
206.48 & 32.42 & -53.28 & 0.4737 E-5
\end{tabular}

Hole 574A

$\begin{array}{rrrr}96.26 & 57.22 & 19.11 & 0.1344 \text { E-5 } \\ 99.26 & 2.57 & 25.44 & 0.3752 \text { E-6 } \\ 102.96 & 11.36 & 15.44 & 0.2959 \text { E-6 } \\ 104.65 & 3.37 & -13.70 & 0.3964 \text { E-6 } \\ 106.15 & 0.10 & -14.01 & 0.1102 \text { E-5 } \\ 112.25 & -27.76 & 16.08 & 0.8613 \text { E-6 } \\ 116.04 & -13.75 & 18.23 & 0.1077 \text { E-5 } \\ 122.71 & 66.28 & 67.55 & 0.8800 \text { E-6 } \\ 125.44 & 5.50 & 16.16 & 0.8511 \text { E-6 } \\ 128.34 & 21.48 & 8.02 & 0.8496 \text { E-6 } \\ 132.03 & 34.39 & 9.02 & 0.1317 \text { E-5 } \\ 135.69 & 37.87 & 23.39 & 0.1267 \text { E-5 } \\ 140.15 & 31.75 & 21.00 & 0.1223 \text { E-5 } \\ 141.89 & 16.96 & 26.89 & 0.1232 \text { E-5 } \\ 144.02 & 64.65 & 0.00 & 0.1219 \text { E-5 } \\ 149.87 & 3.28 & 24.47 & 0.1346 \text { E-5 } \\ 150.27 & 16.27 & 8.68 & 0.1146 \text { E-5 } \\ 150.71 & 15.70 & 6.90 & 0.1248 \text { E-5 } \\ 150.98 & 21.93 & 17.60 & 0.1424 \text { E-5 } \\ 151.37 & 24.16 & 14.25 & 0.1682 \text { E-5 } \\ 151.77 & 45.03 & -34.33 & 0.1335 \text { E-5 } \\ 163.27 & 59.35 & -28.65 & 0.1173 \text { E-5 } \\ 164.77 & -5.12 & -19.41 & 0.8764 \text { E-6 } \\ 166.29 & 60.75 & -30.69 & 0.1191 \text { E-5 } \\ 167.80 & 48.41 & -28.92 & 0.1368 \text { E-5 } \\ 169.30 & 51.77 & -25.34 & 0.1315 \text { E-5 } \\ 170.80 & 64.21 & -33.14 & 0.1182 \text { E-5 } \\ 173.21 & 53.88 & -18.97 & 0.8601 \text { E-6 } \\ 174.71 & 50.05 & -34.16 & 0.1202 \text { E-5 } \\ 176.21 & -89.00 & -0.61 & 0.2962 \text { E-4 } \\ 177.71 & 45.59 & 1.14 & 0.1392 \text { E-5 } \\ 179.21 & 39.35 & -15.31 & 0.1620 \text { E-5 } \\ 180.05 & 41.46 & -4.99 & 0.1458 \text { E-5 }\end{array}$

Hole 574B

$\begin{array}{llrl}186.21 & 43.34 & -5.10 & 0.1609 \text { E-5 } \\ 187.76 & 41.93 & 13.73 & 0.1428 \text { E-5 } \\ 189.11 & 24.59 & 0.60 & 0.2102 \text { E-5 } \\ 190.59 & 40.12 & 3.67 & 0.1511 \text { E-5 } \\ 192.22 & 51.53 & 1.62 & 0.1694 \text { E-5 } \\ 193.71 & 41.94 & 10.06 & 0.1368 \text { E-5 }\end{array}$

Hole 574C

\begin{tabular}{rrrr}
195.18 & 60.55 & -47.36 & 0.8097 E-6 \\
196.48 & 60.42 & -43.81 & 0.8281 E-6 \\
197.06 & 59.90 & -51.40 & 0.1160 E-5 \\
197.84 & 55.94 & -37.40 & 0.1028 E-5 \\
198.12 & 49.85 & -45.53 & 0.9664 E-6 \\
198.43 & 53.28 & -65.64 & 0.9683 E-6 \\
198.83 & 113.16 & -53.32 & 0.7672 E-6 \\
199.21 & 117.42 & -35.87 & 0.1927 E-5 \\
199.61 & -129.81 & -46.78 & 0.1616 E-5 \\
199.93 & 143.11 & -60.74 & 0.5775 E-6 \\
201.61 & -100.36 & -7.76 & 0.1163 E-4 \\
201.91 & -87.73 & -19.54 & 0.1750 E-4 \\
\hline
\end{tabular}


Appendix B. (Continued).

\begin{tabular}{cccc}
\hline $\begin{array}{c}\text { Sub-bottom } \\
\text { depth } \\
(\mathrm{m})\end{array}$ & $\begin{array}{c}\text { Declination } \\
\left({ }^{\circ}\right)\end{array}$ & $\begin{array}{c}\text { Inclination } \\
\left({ }^{\circ}\right)\end{array}$ & $\begin{array}{c}\text { Intensity } \\
(\mathrm{G})\end{array}$ \\
\hline
\end{tabular}

Hole 574C (Cont.)

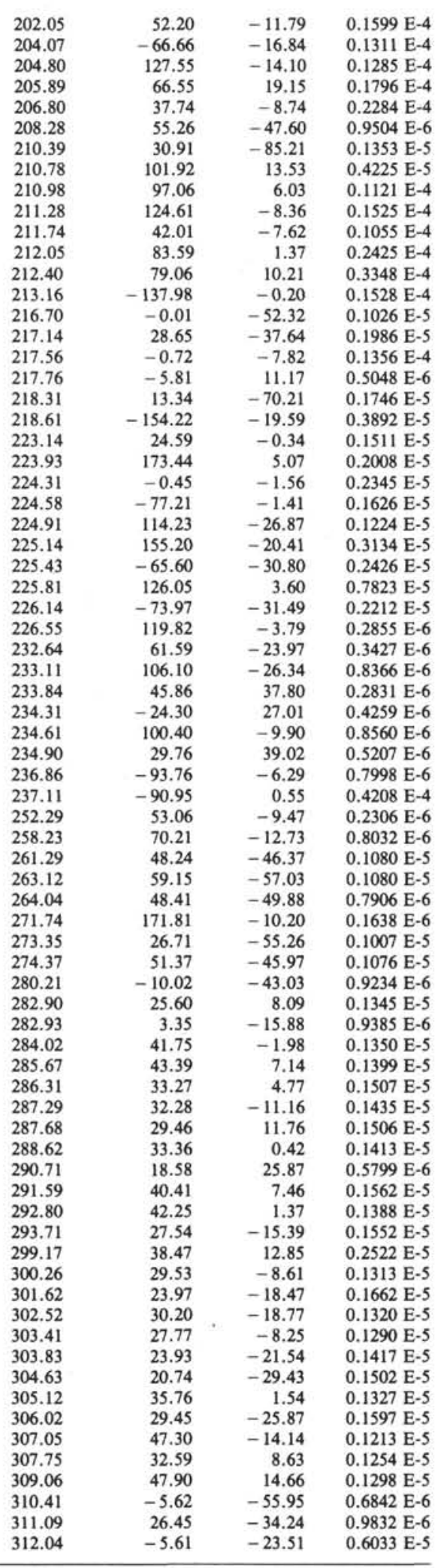

Appendix B. (Continued).

\begin{tabular}{cccc}
\hline $\begin{array}{c}\text { Sub-bottom } \\
\text { depth } \\
(\mathrm{m})\end{array}$ & $\begin{array}{c}\text { Declination } \\
\left({ }^{\circ}\right)\end{array}$ & $\begin{array}{c}\text { Inclination } \\
\left({ }^{\circ}\right)\end{array}$ & $\begin{array}{c}\text { Intensity } \\
(\mathrm{G})\end{array}$ \\
\hline
\end{tabular}

\section{Hole 574C (Cont.)}

\begin{tabular}{|c|c|c|c|}
\hline 312.75 & 2.84 & -41.94 & 0.8577 E-6 \\
\hline 313.94 & -2.14 & -42.67 & 0.7843 E-6 \\
\hline 318.11 & -11.98 & -45.66 & 0.8622 E-6 \\
\hline 320.17 & -19.52 & -43.65 & $0.7608 \mathrm{E}-6$ \\
\hline 320.90 & -1.29 & -32.89 & 0.9536 E-6 \\
\hline 327.72 & 5.57 & -54.42 & 0.6613 E-6 \\
\hline 333.94 & -9.42 & -53.42 & 0.1048 E-5 \\
\hline 336.78 & -5.70 & -34.58 & 0.7271 E-6 \\
\hline 338.25 & -164.93 & -74.77 & 0.1213 E-5 \\
\hline 339.39 & -65.57 & -21.21 & 0.3826 E- 5 \\
\hline 340.85 & 14.24 & -9.68 & 0.1965 E-5 \\
\hline 346.68 & -33.25 & -52.19 & $0.1028 \mathrm{E}-5$ \\
\hline 350.52 & 46.30 & -26.72 & 0.1337 E-5 \\
\hline 351.65 & -122.44 & -24.31 & 0.2946 E-5 \\
\hline 358.33 & 0.31 & -7.65 & 0.4370 E-5 \\
\hline 366.76 & -64.51 & -13.82 & $0.1579 \mathrm{E}-5$ \\
\hline 375.17 & 153.65 & -20.03 & $0.1403 \mathrm{E}-4$ \\
\hline 375.63 & 52.62 & 3.28 & 0.1632 E-4 \\
\hline 376.39 & -22.44 & 24.18 & 0.4732 E-5 \\
\hline 384.57 & -1.62 & 11.65 & 0.1188 E-5 \\
\hline 348.79 & -43.67 & -5.75 & 0.1897 E-6 \\
\hline 385.01 & -15.51 & -1.26 & 0.5421 E-6 \\
\hline 385.78 & -63.01 & -7.23 & 0.9591 E-6 \\
\hline 394.15 & -24.26 & 25.83 & 0.2262 E-5 \\
\hline 395.68 & 103.04 & -48.27 & $0.5252 \mathrm{E}-6$ \\
\hline 396.23 & 87.75 & -6.61 & 0.5772 E- 5 \\
\hline 396.68 & -155.64 & -22.51 & 0.2848 E-5 \\
\hline 403.64 & -38.37 & 22.28 & 0.7695 E-5 \\
\hline 404.97 & 3.51 & 44.37 & 0.1415 E-5 \\
\hline 406.34 & -136.99 & 62.10 & 0.6916 E-6 \\
\hline 408.46 & -55.06 & 43.24 & 0.1312 E-5 \\
\hline 409.92 & 1.83 & 13.98 & 0.1447 E-5 \\
\hline 414.95 & 12.27 & 12.00 & 0.1541 E-5 \\
\hline 416.52 & -22.45 & 13.95 & $0.1383 \mathrm{E}-5$ \\
\hline 418.36 & -99.63 & 20.55 & 0.1317 E-5 \\
\hline 422.76 & 18.26 & 17.62 & 0.1684 E-5 \\
\hline 423.73 & -147.43 & -14.83 & 0.1827 E-5 \\
\hline 432.24 & -63.79 & 17.12 & 0.1981 E-5 \\
\hline 432.90 & -17.03 & 9.66 & 0.4725 E-5 \\
\hline 433.27 & -10.67 & 6.39 & 0.7738 E-5 \\
\hline 434.41 & -48.50 & -3.76 & 0.2565 E-5 \\
\hline 434.86 & -62.87 & 4.55 & 0.3776 E-5 \\
\hline 435.07 & -91.93 & -27.16 & 0.8642 E-6 \\
\hline 435.78 & -167.00 & -16.53 & 0.3390 E-5 \\
\hline 441.65 & -86.57 & 3.77 & 0.1183 E-5 \\
\hline 442.40 & -61.07 & 30.56 & $0.5119 \mathrm{E}-5$ \\
\hline 442.76 & 109.96 & 9.53 & 0.8783 E-5 \\
\hline 443.14 & 7.92 & 58.99 & 0.3506 E-6 \\
\hline 443.81 & 85.22 & 13.28 & 0.5486 E-5 \\
\hline 444.46 & 71.22 & 22.28 & $0.1142 \mathrm{E}-4$ \\
\hline 444.63 & -140.18 & 26.24 & 0.3159 E-5 \\
\hline 445.19 & 127.89 & 34.43 & 0.4085 E-5 \\
\hline 445.84 & 30.72 & 16.50 & 0.3527 E-5 \\
\hline 446.30 & -2.81 & -3.84 & 0.1814 E-5 \\
\hline 451.29 & -139.49 & -16.81 & 0.3040 E-4 \\
\hline 460.57 & 49.47 & 27.35 & 0.2052 E-4 \\
\hline 461.24 & -169.78 & -2.94 & 0.1708 E-4 \\
\hline 461.73 & 80.28 & 6.84 & 0.1323 E-4 \\
\hline 462.11 & 127.51 & 13.31 & 0.8898 E-5 \\
\hline 462.71 & 21.12 & 10.28 & 0.6760 E-5 \\
\hline 463.29 & -64.44 & 23.81 & $0.1332 \mathrm{E}-4$ \\
\hline 463.68 & 178.52 & 22.96 & 0.2193 E-5 \\
\hline 464.28 & -24.65 & 31.18 & 0.1195 E-5 \\
\hline 464.92 & -20.61 & 16.86 & 0.1578 E-5 \\
\hline 470.06 & -52.13 & 7.79 & 0.8996 E-6 \\
\hline 470.40 & 22.56 & 8.73 & 0.2109 E-5 \\
\hline 470.77 & -85.33 & -10.21 & 0.1322 E-5 \\
\hline 479.63 & -75.18 & -29.90 & 0.4698 E-6 \\
\hline 480.03 & 98.18 & -5.03 & 0.1753 E-5 \\
\hline 480.28 & -7.03 & -14.33 & 0.5751 E-6 \\
\hline 480.61 & -10.33 & 15.17 & 0.1388 E-5 \\
\hline 481.13 & -6.61 & 10.35 & 0.1724 E-5 \\
\hline 481.53 & -13.12 & -6.16 & 0.1059 E-5 \\
\hline 481.86 & -37.98 & -21.11 & 0.6720 E-6 \\
\hline
\end{tabular}


Appendix B. (Continued).

\begin{tabular}{cccc}
\hline $\begin{array}{c}\text { Sub-bottom } \\
\text { depth } \\
(\mathrm{m})\end{array}$ & $\begin{array}{c}\text { Declination } \\
\left({ }^{\circ}\right)\end{array}$ & $\begin{array}{c}\text { Inclination } \\
\left({ }^{\circ}\right)\end{array}$ & $\begin{array}{c}\text { Intensity } \\
(\mathrm{G})\end{array}$ \\
\hline
\end{tabular}

Hole 574C (Cont.)

$\begin{array}{lrrr}482.13 & -18.59 & 8.83 & 0.8269 \text { E-6 } \\ 482.86 & -22.85 & 3.41 & 0.2190 \text { E-5 } \\ 489.07 & -5.24 & -14.83 & 0.5807 \text { E-6 } \\ 490.17 & -9.85 & -10.53 & 0.1276 \text { E-5 } \\ 490.84 & -5.15 & 16.89 & 0.1506 \text { E-5 } \\ 491.20 & -6.34 & -3.81 & 0.8450 \text { E-6 } \\ 491.41 & -16.22 & -7.91 & 0.1172 \text { E-5 } \\ 491.86 & -51.99 & -4.37 & 0.4625 \text { E-6 } \\ 492.64 & -6.45 & -0.41 & 0.2556 \text { E-5 } \\ 498.57 & -32.06 & 22.74 & 0.8648 \text { E-6 } \\ 498.77 & -2.99 & -1.98 & 0.1185 \text { E-5 } \\ 499.05 & -1.33 & 23.09 & 0.9921 \text { E-6 } \\ 499.54 & -38.40 & 15.09 & 0.8277 \text { E-6 } \\ 499.97 & -8.78 & 25.79 & 0.1269 \text { E-5 } \\ 500.23 & -11.81 & 24.94 & 0.6042 \text { E-6 } \\ 500.60 & -30.62 & 27.16 & 0.7612 \text { E-6 } \\ 500.99 & -51.21 & 38.99 & 0.6702 \text { E-6 } \\ 501.37 & 13.20 & -30.67 & 0.8634 \text { E-6 } \\ 501.70 & -17.76 & -0.08 & 0.1623 \text { E-5 } \\ 502.19 & -28.28 & 1.53 & 0.5164 \text { E-6 } \\ 502.72 & -90.72 & 6.35 & 0.1302 \text { E-5 } \\ 503.16 & -76.54 & 19.96 & 0.1533 \text { E-5 } \\ 503.40 & -0.47 & 10.48 & 0.2513 \text { E-5 } \\ 503.93 & -14.59 & 15.38 & 0.4226 \text { E-5 } \\ 504.40 & 121.50 & 66.10 & 0.4311 \text { E-4 } \\ 504.77 & -108.40 & -13.90 & 0.7935 \text { E-4 } \\ 505.05 & 101.60 & -65.70 & 0.4581 \text { E-4 } \\ 505.23 & -133.10 & -63.80 & 0.7238 \text { E-4 } \\ 505.92 & -103.20 & -22.40 & 0.5667 \text { E-4 } \\ 508.40 & 96.80 & -07.00 & 0.7053 \text { E-4 } \\ 508.72 & 101.70 & 37.30 & 0.3732 \text { E-4 } \\ 509.05 & -104.90 & -04.60 & 0.1367 \text { E-4 } \\ 509.39 & -92.80 & -48.70 & 0.5195 \text { E-4 } \\ 509.76 & 98.00 & 24.30 & 0.1961 \text { E-4 } \\ 510.30 & 0.98 & 8.18 & 0.2011 \text { E-4 } \\ 510.61 & -154.19 & 26.19 & 0.1264 \text { E-4 } \\ 517.57 & 78.00 & 63.20 & 0.8394 \text { E-4 } \\ 518.48 & 69.10 & 53.60 & 0.8369 \text { E-4 } \\ 518.77 & 93.50 & 55.70 & 0.7338 \text { E-4 } \\ 519.52 & -077.20 & -37.40 & 0.2965 \text { E-5 } \\ 520.00 & -085.40 & -21.60 & 0.4318 \text { E-4 }\end{array}$

Hole 575

$\begin{array}{rrrl}35.31 & -132.23 & -40.33 & 0.3275 \text { E-5 } \\ 35.91 & 30.01 & 1.64 & 0.7878 \text { E-6 } \\ 36.38 & 30.57 & -33.43 & 0.2323 \text { E-5 } \\ 36.81 & 6.00 & -13.37 & 0.4402 \text { E-5 } \\ 37.39 & 44.58 & -72.20 & 0.5699 \text { E-6 } \\ 37.88 & 16.75 & -18.17 & 0.2509 \text { E-5 } \\ 38.31 & 123.23 & -75.13 & 0.7707 \text { E-6 } \\ 38.81 & 35.06 & -16.40 & 0.4074 \text { E-5 } \\ 39.38 & 15.85 & -17.24 & 0.5058 \text { E-5 } \\ 39.81 & 29.11 & -4.70 & 0.5978 \text { E-5 } \\ 40.31 & 15.28 & 6.54 & 0.1040 \text { E-4 } \\ 41.31 & -165.24 & 4.31 & 0.8456 \text { E-5 } \\ 41.81 & -171.23 & -1.21 & 0.6947 \text { E-5 } \\ 42.38 & 0.68 & -5.84 & 0.5870 \text { E-5 } \\ 42.81 & 0.45 & 0.60 & 0.4508 \text { E-5 } \\ 43.31 & 5.14 & -14.79 & 0.2149 \text { E-5 } \\ 43.88 & 168.28 & -2.33 & 0.4982 \text { E-5 } \\ 44.31 & -174.24 & 1.96 & 0.3889 \text { E-5 } \\ 44.81 & 65.25 & -38.57 & 0.1766 \text { E-5 } \\ 45.31 & 68.29 & -1.37 & 0.1177 \text { E-5 } \\ 45.88 & 145.21 & -8.08 & 0.2724 \text { E-5 } \\ 46.31 & 90.55 & -7.71 & 0.2369 \text { E-5 } \\ 46.81 & 82.22 & -13.13 & 0.1981 \text { E-5 } \\ 47.38 & 68.71 & -19.75 & 0.1242 \text { E-5 } \\ 47.81 & -13.25 & -28.74 & 0.8124 \text { E-6 } \\ 48.31 & -1.18 & -20.98 & 0.6653 \text { E-6 } \\ 48.88 & -9.99 & -33.96 & 0.7818 \text { E-6 } \\ 49.25 & 1.15 & -32.17 & 0.8997 \text { E-6 } \\ 49.74 & 23.17 & -39.85 & 0.4713 \text { E-6 } \\ 50.38 & 27.19 & -59.34 & 0.1331 \text { E-5 }\end{array}$

Appendix B. (Continued).

\begin{tabular}{cccc}
\hline $\begin{array}{c}\text { Sub-bottom } \\
\text { depth }\end{array}$ & $\begin{array}{c}\text { Declination } \\
(\mathrm{m})\end{array}$ & $\begin{array}{c}\text { Inclination } \\
\left({ }^{\circ}\right)\end{array}$ & $\begin{array}{c}\text { Intensity } \\
\left({ }^{\circ}\right)\end{array}$ \\
\hline
\end{tabular}

Hole 575 (Cont.)

\begin{tabular}{|c|c|c|c|}
\hline 50.81 & 1.89 & -23.08 & 0.4949 E-6 \\
\hline 51.31 & 19.28 & -45.25 & 0.6055 E-6 \\
\hline 51.88 & -14.80 & -21.33 & 0.8629 E-6 \\
\hline 52.31 & -8.19 & -15.71 & 0.6173 E-6 \\
\hline 52.81 & 2.93 & -52.25 & 0.4420 E-6 \\
\hline 53.38 & 21.92 & -12.56 & 0.5383 E-6 \\
\hline 53.81 & 27.90 & -31.70 & 0.5439 E- 6 \\
\hline 54.31 & 23.53 & -10.51 & 0.5020 E-6 \\
\hline 54.81 & -12.35 & -23.90 & 0.9652 E-6 \\
\hline 55.31 & 0.29 & -39.52 & 0.9079 E-6 \\
\hline 55.81 & -9.21 & -36.53 & 0.9259 E-6 \\
\hline 56.81 & -23.30 & -22.30 & 0.1030 E-5 \\
\hline 57.31 & -8.86 & -20.84 & 0.7780 E-6 \\
\hline 57.81 & -6.62 & -12.77 & $0.8860 \mathrm{E}-6$ \\
\hline 58.31 & -12.70 & -31.21 & 0.6390 E-6 \\
\hline 58.81 & -25.28 & -8.84 & 0.5837 E-6 \\
\hline 59.31 & -147.25 & -68.27 & $0.3380 \mathrm{E}-6$ \\
\hline 59.81 & -14.78 & -25.54 & $0.5255 \mathrm{E}-6$ \\
\hline 60.31 & 15.64 & 10.33 & 0.6936 E-6 \\
\hline 60.81 & -12.53 & -16.07 & 0.6049 E-6 \\
\hline 61.31 & -27.53 & -35.50 & 0.4338 E-6 \\
\hline 61.81 & -52.16 & -40.55 & $0.3788 \mathrm{E}-6$ \\
\hline 62.81 & -15.23 & -2.99 & 0.6925 E-6 \\
\hline 63.81 & -25.07 & -18.11 & 0.9862 E-6 \\
\hline 64.61 & -20.47 & -25.52 & 0.8706 E-6 \\
\hline 65.31 & -13.54 & -14.16 & 0.8028 E-6 \\
\hline 66.11 & -17.86 & -28.15 & 0.5747 E-6 \\
\hline 66.81 & 30.21 & -15.42 & 0.4612 E-6 \\
\hline 67.61 & -13.40 & -33.70 & 0.7146 E-6 \\
\hline 68.31 & -47.45 & -11.13 & 0.1023 E-5 \\
\hline 69.11 & -61.65 & -6.77 & 0.5349 E-6 \\
\hline 69.81 & -50.61 & -17.31 & 0.8744 E-6 \\
\hline 70.81 & -146.23 & -8.01 & 0.3055 E- 5 \\
\hline 71.31 & -50.51 & -20.75 & 0.9244 E-6 \\
\hline 72.31 & -167.56 & 12.88 & $0.1068 \mathrm{E}-4$ \\
\hline 73.11 & 112.81 & 15.29 & 0.3307 E-4 \\
\hline 73.46 & 114.11 & 7.62 & $0.2180 \mathrm{E}-4$ \\
\hline 73.86 & 109.84 & -20.94 & 0.2048 E-5 \\
\hline 74.06 & 80.60 & 1.14 & 0.2191 E-5 \\
\hline 74.61 & 87.13 & -37.73 & 0.2834 E-5 \\
\hline 75.01 & 88.99 & 4.44 & 0.5569 E-5 \\
\hline 75.56 & 49.13 & -44.97 & 0.4593 E-5 \\
\hline 75.96 & 83.77 & -22.12 & 0.4000 E-5 \\
\hline 76.11 & 73.25 & -2.26 & 0.3183 E-5 \\
\hline 76.71 & -93.09 & -12.89 & 07775 E-5 \\
\hline 77.06 & -103.64 & -5.71 & 0.6628 E-5 \\
\hline 77.46 & -75.88 & -37.91 & 0.1364 E-5 \\
\hline 78.01 & 55.09 & 7.18 & 0.3255 E-5 \\
\hline 78.56 & 58.85 & 1.60 & $0.2543 \mathrm{E}-4$ \\
\hline 78.96 & 52.10 & -5.54 & 0.3630 E-5 \\
\hline 79.30 & 68.71 & -0.51 & 0.4731 E-5 \\
\hline 79.93 & -117.90 & -35.53 & 0.7902 E-5 \\
\hline 84.51 & 171.22 & -25.81 & 0.4558 E-5 \\
\hline 85.01 & 4.01 & -11.51 & 0.9678 E-6 \\
\hline 85.62 & -30.33 & -41.84 & 0.3782 E-6 \\
\hline 85.99 & -26.42 & -34.29 & 0.5776 E-6 \\
\hline 86.51 & -26.55 & -2.19 & 0.3996 E-6 \\
\hline 87.06 & -19.90 & -26.83 & 0.5451 E-6 \\
\hline 87.49 & -12.94 & -18.76 & 0.5183 E-6 \\
\hline
\end{tabular}

Hole 575A

$\begin{array}{lrrr}94.09 & 46.91 & -14.27 & 0.4029 \text { E-5 } \\ 94.49 & 50.13 & -3.48 & 0.9490 \text { E-5 } \\ 95.09 & 56.08 & 2.89 & 0.6587 \text { E-5 } \\ 95.59 & 62.36 & -5.36 & 0.8648 \text { E-5 } \\ 95.99 & -97.36 & -19.58 & 0.6722 \text { E-5 } \\ 96.59 & -102.62 & -3.36 & 0.6496 \text { E-5 } \\ 97.09 & -86.56 & 35.92 & 0.7884 \text { E-5 } \\ 97.49 & 60.58 & 21.11 & 0.1183 \text { E-4 } \\ 98.09 & 71.76 & 1.55 & 0.3652 \text { E-4 } \\ 98.99 & 4.54 & -3.42 & 0.1298 \text { E-4 } \\ 99.39 & 28.45 & 1.57 & 0.3044 \text { E-5 } \\ 99.99 & -166.22 & -20.38 & 0.9745 \text { E-5 }\end{array}$


Appendix B. (Continued).

\begin{tabular}{cccc}
\hline $\begin{array}{c}\text { Sub-bottom } \\
\text { depth } \\
(\mathrm{m})\end{array}$ & $\begin{array}{c}\text { Declination } \\
\left({ }^{\circ}\right)\end{array}$ & $\begin{array}{c}\text { Inclination } \\
\left({ }^{\circ}\right)\end{array}$ & $\begin{array}{c}\text { Intensity } \\
(\mathrm{G})\end{array}$ \\
\hline
\end{tabular}

Hole 575A (Cont.)

\begin{tabular}{|c|c|c|c|}
\hline 100.49 & -164.99 & -3.88 & $0.1021 \mathrm{E}-4$ \\
\hline 100.89 & -163.03 & -13.43 & $0.2448 \mathrm{E}-4$ \\
\hline 101.49 & -163.62 & 3.98 & 0.5921 E-5 \\
\hline 101.99 & 5.40 & 16.31 & 0.2991 E-5 \\
\hline 102.39 & 137.23 & -25.54 & 0.2033 E- 5 \\
\hline 102.89 & 6.25 & 8.22 & $0.1458 \mathrm{E}-4$ \\
\hline 103.29 & 7.11 & -1.49 & $0.2232 \mathrm{E}-4$ \\
\hline 103.89 & 3.44 & 3.39 & $0.1986 \mathrm{E}-4$ \\
\hline 104.39 & 3.63 & 2.60 & 0.8409 E-5 \\
\hline 104.79 & -5.49 & -3.87 & $0.6198 \mathrm{E}-5$ \\
\hline 105.87 & -25.75 & -19.98 & $0.7246 \mathrm{E}-5$ \\
\hline 106.49 & -20.50 & -3.84 & $0.6080 \mathrm{E}-5$ \\
\hline 106.99 & -30.44 & 2.43 & 0.1126 E-4 \\
\hline 107.39 & -21.15 & -20.45 & 0.4619 E-5 \\
\hline 107.99 & -19.55 & -33.84 & $0.4231 \mathrm{E}-5$ \\
\hline 108.54 & -24.50 & -4.23 & $0.2497 \mathrm{E}-4$ \\
\hline 108.89 & -24.33 & -0.43 & 0.8369 E-5 \\
\hline 109.49 & -18.87 & -0.17 & 0.6972 E-5 \\
\hline 110.79 & -164.29 & -3.49 & $0.1832 \mathrm{E}-4$ \\
\hline 111.19 & -154.46 & -16.78 & 0.3999 E-5 \\
\hline 111.79 & -126.48 & -18.25 & 0.2192 E-5 \\
\hline 112.29 & -169.33 & 14.68 & 0.5741 E-5 \\
\hline 112.69 & 177.97 & -2.64 & $0.2623 \mathrm{E}-4$ \\
\hline 113.29 & 153.35 & -69.57 & 0.4002 E-5 \\
\hline 113.87 & -26.07 & -23.92 & 0.7485 E-5 \\
\hline 114.39 & 142.97 & -10.88 & $0.1279 \mathrm{E}-4$ \\
\hline 114.79 & -44.85 & 11.02 & $0.1783 \mathrm{E}-4$ \\
\hline 115.39 & -42.33 & 2.94 & $0.1678 \mathrm{E}-4$ \\
\hline 115.89 & -48.54 & 8.38 & $0.8713 \mathrm{E}-5$ \\
\hline 116.29 & -20.22 & 42.01 & $0.1532 \mathrm{E}-4$ \\
\hline 116.89 & -47.25 & 0.97 & 0.9641 E-5 \\
\hline 117.39 & -39.11 & 12.09 & 0.2598 E-5 \\
\hline 117.79 & -48.58 & -9.06 & $0.5359 \mathrm{E}-5$ \\
\hline 118.39 & 131.96 & 1.61 & 0.5687 E-5 \\
\hline 118.89 & 119.40 & -25.28 & $0.3092 \mathrm{E}-5$ \\
\hline 119.17 & 133.43 & -26.52 & 0.3820 E-5 \\
\hline 119.69 & 136.97 & 0.21 & 0.9366 E-5 \\
\hline 120.19 & 173.42 & 2.25 & $0.4720 \mathrm{E}-5$ \\
\hline 120.59 & 147.86 & 3.93 & $0.1153 \mathrm{E}-4$ \\
\hline 121.19 & 166.54 & -12.30 & 0.1347 E-5 \\
\hline 121.69 & 144.69 & -6.23 & 0.5368 E-5 \\
\hline 122.09 & 135.63 & -28.00 & $0.1082 \mathrm{E}-4$ \\
\hline 122.69 & 138.85 & -0.81 & $0.1277 \mathrm{E}-4$ \\
\hline 123.19 & 138.02 & 4.02 & $0.1580 \mathrm{E}-4$ \\
\hline 123.39 & -77.57 & -34.25 & $0.6874 \mathrm{E}-5$ \\
\hline 123.79 & -70.72 & -19.20 & 0.6944 E-5 \\
\hline 124.39 & -70.40 & -18.80 & $0.2046 \mathrm{E}-5$ \\
\hline 124.89 & -84.47 & -6.00 & 0.7238 E-5 \\
\hline 125.29 & -66.81 & -36.58 & $0.3555 \mathrm{E}-5$ \\
\hline 125.89 & 94.03 & 4.88 & $0.3131 \mathrm{E}-5$ \\
\hline 126.59 & -7.00 & -62.15 & 0.5840 E-5 \\
\hline 127.09 & -34.14 & -21.30 & 0.1440 E-5 \\
\hline 127.49 & -33.97 & -3.81 & $0.1026 \mathrm{E}-4$ \\
\hline 128.03 & -31.33 & -18.84 & $0.4055 \mathrm{E}-5$ \\
\hline 128.21 & -35.19 & 19.16 & $0.1060 \mathrm{E}-4$ \\
\hline 128.66 & 122.84 & -17.65 & 0.4536 E-6 \\
\hline 129.11 & 132.28 & -81.47 & 0.1043 E-5 \\
\hline 129.54 & 140.63 & 6.25 & 0.2655 E-5 \\
\hline 130.11 & -92.24 & 0.52 & $0.4117 \mathrm{E}-4$ \\
\hline 130.61 & 125.30 & -32.15 & 0.5678 E-6 \\
\hline 131.04 & 12.80 & -31.12 & 0.4453 E- 6 \\
\hline 131.61 & 28.53 & -60.73 & 0.4245 E-6 \\
\hline 132.26 & -14.25 & -15.57 & 0.3794 E-6 \\
\hline 132.76 & -9.03 & -29.52 & 0.9063 E-6 \\
\hline 133.26 & -17.55 & -20.18 & 0.6959 E-6 \\
\hline 133.76 & -89.93 & 1.77 & 0.4232 E-4 \\
\hline 134.76 & -17.57 & -28.50 & 0.6724 E-6 \\
\hline 135.42 & -22.97 & -67.61 & 0.9312 E-6 \\
\hline 135.80 & 8.91 & -8.42 & 0.1559 E-5 \\
\hline 136.41 & 87.80 & -1.30 & $0.4177 \mathrm{E}-4$ \\
\hline 136.73 & -16.90 & -31.54 & 0.6977 E-6 \\
\hline 137.31 & 64.47 & -54.39 & 0.1251 E-5 \\
\hline 137.91 & -90.20 & 0.37 & $0.4224 \mathrm{E}-4$ \\
\hline 138.26 & -18.09 & -23.70 & 0.6456 E-6 \\
\hline
\end{tabular}

Appendix B. (Continued).

\begin{tabular}{|c|c|c|}
\hline $\begin{array}{l}\text { Sub-bottom } \\
\text { depth } \\
\text { (m) }\end{array}$ & $\begin{array}{c}\text { Declination } \\
\left({ }^{\circ}\right)\end{array}$ & $\begin{array}{c}\text { Inclination } \\
\left({ }^{\circ}\right)\end{array}$ \\
\hline
\end{tabular}

\section{Hole 575A (Cont.)}

\begin{tabular}{|c|c|c|c|}
\hline 139.01 & 6.11 & -45.89 & 0.6391 E-6 \\
\hline 139.95 & -46.26 & -6.20 & 0.2427 E-5 \\
\hline 140.46 & -25.90 & -28.55 & 0.8430 E-6 \\
\hline 141.04 & -30.42 & -40.59 & 0.9949 E-6 \\
\hline 141.44 & -24.37 & -33.18 & 0.6581 E- 6 \\
\hline 141.96 & -14.63 & -38.48 & 0.7713 E- 6 \\
\hline 142.71 & -131.56 & -5.92 & 0.5062 E-5 \\
\hline 143.20 & -15.68 & -31.65 & 0.8875 E-6 \\
\hline 143.71 & -17.00 & -13.29 & 0.8446 E-6 \\
\hline 144.26 & -30.83 & -69.62 & 0.1275 E-5 \\
\hline 144.86 & -8.15 & -18.29 & 0.6746 E-6 \\
\hline 145.16 & -16.56 & -9.83 & 0.5855 E-6 \\
\hline 145.61 & -12.11 & -16.73 & 0.6973 E-6 \\
\hline 145.76 & 126.20 & -31.37 & $0.2952 \mathrm{E}-7$ \\
\hline 146.06 & 35.00 & -5.66 & 0.1235 E-5 \\
\hline 147.11 & -7.89 & -21.31 & 0.1222 E-5 \\
\hline 147.66 & -13.20 & -17.08 & 0.6800 E-6 \\
\hline 148.01 & -10.93 & -37.50 & 0.1335 E-5 \\
\hline 149.81 & -5.64 & -37.66 & 0.4633 E- 6 \\
\hline 150.35 & -25.56 & -37.34 & $0.6518 \mathrm{E}-6$ \\
\hline 150.96 & 0.99 & 15.91 & 0.9398 E-6 \\
\hline 151.31 & -19.04 & -20.17 & 0.7237 E-6 \\
\hline 151.71 & -16.26 & -22.43 & 0.8365 E-6 \\
\hline 152.06 & -15.51 & -31.34 & 0.6051 E-6 \\
\hline 152.46 & -9.02 & -33.64 & $0.6866 \mathrm{E}-6$ \\
\hline 152.96 & -11.91 & -29.47 & 0.7326 E-6 \\
\hline 153.16 & -5.81 & -25.20 & 0.7660 E-6 \\
\hline 153.51 & -12.97 & -26.34 & 0.7771 E-6 \\
\hline 153.96 & -10.34 & -39.50 & 0.8570 E-6 \\
\hline 154.31 & -30.10 & -40.18 & 0.8498 E-6 \\
\hline 154.86 & -14.95 & -18.39 & 0.9572 E-6 \\
\hline 155.31 & -4.72 & -36.31 & 0.6784 E-6 \\
\hline 155.66 & -22.36 & -34.28 & 0.5156 E-6 \\
\hline 156.06 & -22.98 & -23.17 & 0.9095 E-6 \\
\hline 156.46 & -10.22 & -42.70 & 0.7463 E-6 \\
\hline 157.31 & -21.97 & -40.22 & 0.5130 E-6 \\
\hline 157.71 & -10.03 & -4.24 & 0.3095 E-6 \\
\hline 158.11 & 54.24 & -35.22 & 0.1181 E-5 \\
\hline 158.61 & 66.83 & -48.62 & 0.1454 E-5 \\
\hline 159.16 & 89.50 & -64.41 & 0.1348 E-5 \\
\hline 159.76 & 17.70 & -74.18 & 0.1455 E-5 \\
\hline 160.11 & 19.43 & 2.61 & 0.9537 E-6 \\
\hline 160.51 & -34.77 & -72.29 & 0.1509 E-5 \\
\hline 161.11 & 42.96 & -16.53 & 0.1177 E-5 \\
\hline 161.66 & 60.39 & -70.93 & $0.1698 \mathrm{E}-5$ \\
\hline 162.26 & 36.37 & -11.38 & $0.1378 \mathrm{E}-5$ \\
\hline 162.61 & 27.53 & -14.02 & 0.1531 E-5 \\
\hline 163.16 & -31.42 & -1.02 & 0.1044 E-5 \\
\hline 163.76 & -38.36 & -12.32 & 0.4906 E-6 \\
\hline 163.96 & -9.71 & -50.92 & 0.6072 E-6 \\
\hline 164.51 & -28.62 & -62.75 & 0.7096 E-6 \\
\hline 164.99 & 11.40 & -27.43 & 0.8673 E-6 \\
\hline 165.39 & 17.43 & -34.06 & 0.6178 E-6 \\
\hline 165.99 & 2.22 & -21.21 & 0.7960 E-6 \\
\hline 166.49 & 12.86 & -21.48 & 0.1490 E-5 \\
\hline 166.89 & 3.33 & -30.88 & 0.1469 E-5 \\
\hline 167.49 & 6.52 & -27.04 & 0.1054 E-5 \\
\hline 167.99 & -6.19 & -55.41 & 0.1181 E-5 \\
\hline 168.39 & 14.47 & -50.37 & 0.6947 E-6 \\
\hline 168.79 & -4.35 & -44.73 & 0.5452 E-6 \\
\hline 169.39 & -8.16 & -34.11 & 0.7868 E-6 \\
\hline 169.89 & -9.31 & -29.47 & 0.4717 E-6 \\
\hline 170.29 & -12.21 & -36.50 & 0.8016 E-6 \\
\hline 170.89 & 81.88 & -4.26 & $0.6930 \mathrm{E}-5$ \\
\hline 171.39 & -13.43 & -41.40 & $0.6439 \mathrm{E}-6$ \\
\hline 171.79 & -12.51 & -37.82 & 0.9849 E-6 \\
\hline 172.39 & -3.64 & -52.40 & 0.7415 E-6 \\
\hline 172.79 & -5.10 & -41.37 & 0.9638 E-6 \\
\hline 173.39 & -7.78 & -45.22 & 0.6168 E-6 \\
\hline 174.29 & -19.21 & -41.25 & 0.8716 E-6 \\
\hline 175.49 & -13.88 & -70.59 & 0.5769 E-6 \\
\hline 175.99 & -39.15 & -64.36 & 0.1071 E-5 \\
\hline 176.39 & -15.47 & -39.26 & 0.9960 E-6 \\
\hline 176.89 & -2.89 & -38.15 & 0.7263 E-6 \\
\hline
\end{tabular}


Appendix B. (Continued).

\begin{tabular}{|c|c|c|c|}
\hline $\begin{array}{l}\text { Sub-bottom } \\
\text { depth } \\
\text { (m) }\end{array}$ & $\begin{array}{c}\text { Declination } \\
\left({ }^{\circ}\right)\end{array}$ & $\begin{array}{c}\text { Inclination } \\
\left({ }^{\circ}\right)\end{array}$ & $\begin{array}{l}\text { Intensity } \\
\text { (G) }\end{array}$ \\
\hline
\end{tabular}

Hole 575A (Cont.)

$\begin{array}{lrrr}177.49 & 28.18 & -44.11 & 0.7518 \mathrm{E}-6 \\ 177.89 & -18.89 & -38.70 & 0.6630 \mathrm{E}-6 \\ 178.49 & 3.44 & -49.64 & 0.6612 \mathrm{E}-6 \\ 178.99 & -1.42 & -42.86 & 0.8350 \mathrm{E}-6 \\ 179.99 & -10.18 & -42.37 & 0.8043 \mathrm{E}-6 \\ 180.89 & -5.95 & -69.22 & 0.8150 \mathrm{E}-6 \\ 181.49 & 16.59 & -64.68 & 0.6790 \mathrm{E}-6 \\ 182.19 & -4.05 & -59.13 & 0.8538 \mathrm{E}-6 \\ 183.69 & 0.49 & -14.23 & 0.4606 \mathrm{E}-6 \\ 184.59 & -2.61 & -19.76 & 0.4839 \mathrm{E}-6 \\ 186.19 & 10.47 & -26.50 & 0.4911 \mathrm{E}-6 \\ 187.69 & 6.90 & -30.06 & 0.5668 \mathrm{E}-6 \\ 188.99 & 11.86 & -45.23 & 0.8030 \mathrm{E}-6 \\ 189.99 & -3.08 & -41.56 & 0.1133 \mathrm{E}-5 \\ 193.39 & 2.70 & -41.90 & 0.7166 \mathrm{E}-6 \\ 195.79 & -9.15 & -43.40 & 0.8092 \mathrm{E}-6 \\ 198.31 & -2.23 & -42.84 & 0.8488 \mathrm{E}-6 \\ 199.76 & -2.90 & -40.86 & 0.7369 \mathrm{E}-6 \\ 201.52 & 11.96 & -46.23 & 0.8036 \mathrm{E}-6 \\ 202.41 & -14.28 & -49.89 & 0.8254 \mathrm{E}-6 \\ 205.01 & -3.00 & -39.99 & 0.1015 \mathrm{E}-5 \\ 205.41 & -9.15 & -49.72 & 0.9256 \mathrm{E}-6 \\ 206.91 & 9.10 & -40.05 & 0.5588 \mathrm{E}-6\end{array}$

Hole 575B

\begin{tabular}{|c|c|c|c|}
\hline 39.51 & -79.29 & -21.35 & $0.2461 \mathrm{E}-4$ \\
\hline 39.76 & -68.60 & -10.73 & $0.2979 \mathrm{E}-4$ \\
\hline 40.01 & -56.86 & 10.94 & $0.2714 \mathrm{E}-4$ \\
\hline 40.24 & -57.44 & -5.41 & $0.2191 \mathrm{E}-4$ \\
\hline 40.47 & -57.02 & 1.06 & $0.2174 \mathrm{E}-4$ \\
\hline 40.60 & -60.05 & -0.64 & $0.2301 \mathrm{E}-4$ \\
\hline 40.86 & -60.88 & 3.86 & $0.2307 \mathrm{E}-4$ \\
\hline 41.09 & -55.41 & 3.40 & $0.2023 \mathrm{E}-4$ \\
\hline 41.36 & -55.00 & 2.12 & 0.1724 E-4 \\
\hline 41.61 & -52.92 & -5.51 & $0.1235 \mathrm{E}-4$ \\
\hline 41.86 & -75.09 & -5.88 & $0.2153 \mathrm{E}-4$ \\
\hline 42.10 & -67.92 & -7.98 & $0.2929 \mathrm{E}-4$ \\
\hline 42.36 & -65.75 & 1.24 & $0.3816 \mathrm{E}-4$ \\
\hline 42.59 & -43.03 & -5.58 & $0.6883 \mathrm{E}-5$ \\
\hline 42.86 & -4.82 & 38.21 & $0.2249 \mathrm{E}-5$ \\
\hline 43.11 & -13.51 & 40.94 & 0.2964 E-5 \\
\hline 43.36 & -14.59 & 7.86 & 0.2973 E-5 \\
\hline 43.60 & 112.07 & -3.31 & $0.5084 \mathrm{E}-5$ \\
\hline 43.86 & -38.65 & 4.78 & 0.1060 E-4 \\
\hline 44.09 & -23.02 & 7.86 & 0.6013 E-5 \\
\hline 44.36 & -96.49 & 0.72 & 0.6921 E-5 \\
\hline 44.61 & -76.42 & -8.74 & $0.1251 \mathrm{E}-4$ \\
\hline 44.86 & -74.93 & -5.38 & 0.3289 E-5 \\
\hline 45.10 & -46.73 & 7.09 & 0.3172 E-5 \\
\hline 45.36 & -21.72 & 5.83 & 0.1885 E-5 \\
\hline 45.59 & -26.12 & 9.05 & 0.2234 E-5 \\
\hline 45.86 & -3.28 & 33.20 & 0.2271 E-5 \\
\hline 46.11 & 106.79 & 1.05 & 0.1273 E-5 \\
\hline 46.36 & 100.76 & 29.05 & 0.1186 E-5 \\
\hline 46.60 & 69.03 & 6.34 & 0.5959 E-6 \\
\hline 46.86 & -121.47 & 22.26 & 0.2994 E-5 \\
\hline 47.09 & -94.56 & 18.45 & 0.3989 E-5 \\
\hline 47.36 & -73.05 & 17.69 & 0.2109 E-5 \\
\hline 47.61 & -49.99 & 47.70 & 0.3226 E-5 \\
\hline 47.86 & -26.67 & 23.84 & 0.1326 E-5 \\
\hline 48.46 & -24.59 & -28.28 & 0.4139 E-5 \\
\hline 48.75 & -11.40 & -4.03 & 0.2573 E-5 \\
\hline 49.16 & -23.53 & -6.11 & 0.4615 E-5 \\
\hline 49.46 & -9.45 & -44.96 & 0.4950 E-5 \\
\hline 49.66 & -41.85 & -1.12 & 0.4654 E-5 \\
\hline 49.96 & -70.19 & 26.48 & 0.4693 E- 5 \\
\hline 50.28 & -66.58 & 21.64 & 0.3239 E- 5 \\
\hline 50.56 & -56.51 & 26.60 & 0.2572 E-5 \\
\hline 50.86 & 53.54 & 29.52 & 0.2446 E-5 \\
\hline 51.16 & -39.21 & 17.79 & 0.2284 E-5 \\
\hline 51.46 & 76.65 & -17.21 & 0.1847 E-5 \\
\hline 51.78 & 50.38 & -32.39 & 0.1072 E-5 \\
\hline 52.06 & 70.34 & 19.41 & 0.1518 E-5 \\
\hline
\end{tabular}

Appendix B. (Continued).

\begin{tabular}{cccc}
$\begin{array}{c}\text { Sub-bottom } \\
\text { depth }\end{array}$ & $\begin{array}{c}\text { Declination } \\
(\mathrm{m})\end{array}$ & $\begin{array}{c}\text { Inclination } \\
\left({ }^{\circ}\right)\end{array}$ & $\begin{array}{c}\text { Intensity } \\
\left({ }^{\circ}\right)\end{array}$ \\
\hline
\end{tabular}

Hole 575B (Cont.)

\begin{tabular}{|c|c|c|c|}
\hline 52.36 & 54.27 & 37.69 & 0.2865 E-5 \\
\hline 52.66 & 24.37 & 29.18 & 0.1860 E-5 \\
\hline 52.96 & 138.50 & 36.09 & 0.3997 E-5 \\
\hline 53.28 & 114.24 & 73.40 & 0.6093 E-5 \\
\hline 53.56 & 81.35 & 20.54 & 0.4892 E- 5 \\
\hline 53.86 & -52.34 & 6.56 & 0.9651 E-5 \\
\hline 54.16 & -54.80 & 4.65 & $0.1058 \mathrm{E}-4$ \\
\hline 54.46 & -46.47 & 28.63 & $0.1040 \mathrm{E}-4$ \\
\hline 54.78 & -4.19 & 10.10 & 0.1339 E-5 \\
\hline 55.06 & 74.24 & 5.10 & 0.2921 E- 5 \\
\hline 55.66 & 4.06 & 13.41 & 0.2414 E- 5 \\
\hline 56.16 & 107.71 & 7.33 & 0.2675 E- 5 \\
\hline 56.46 & -43.31 & 21.95 & 0.4141 E-5 \\
\hline 56.76 & -42.15 & 9.45 & $0.1069 \mathrm{E}-4$ \\
\hline 57.16 & -30.07 & 11.20 & 0.5584 E-5 \\
\hline 57.46 & -22.08 & 1.84 & 0.7635 E-5 \\
\hline 57.76 & -10.18 & 2.09 & 0.2852 E- 5 \\
\hline 58.06 & -6.04 & 4.77 & 0.2567 E- 5 \\
\hline 58.36 & 2.29 & $19.29^{\circ}$ & 0.1752 E-5 \\
\hline 58.66 & -7.70 & 38.37 & 0.1918 E-5 \\
\hline 58.96 & -19.97 & 5.44 & 0.2862 E- 5 \\
\hline 59.26 & -0.11 & -2.10 & 0.3586 E-5 \\
\hline 59.56 & 9.13 & 16.12 & 0.3122 E-5 \\
\hline 59.86 & 3.15 & 17.58 & 0.2341 E- 5 \\
\hline 60.16 & -6.18 & 31.90 & 0.2400 E-5 \\
\hline 60.46 & 9.23 & 20.67 & 0.2117 E-5 \\
\hline 60.76 & 7.63 & 31.78 & $0.2346 \mathrm{E}-5$ \\
\hline 61.06 & 1.84 & -5.88 & $0.6080 \mathrm{E}-6$ \\
\hline 61.36 & -18.70 & -2.21 & 0.6506 E-6 \\
\hline 61.66 & -1.68 & 11.85 & 0.1143 E-5 \\
\hline 61.96 & -16.55 & 6.49 & 0.9023 E-6 \\
\hline 62.26 & 2.11 & 10.18 & 0.6143 E-6 \\
\hline 62.56 & -7.08 & 6.71 & 0.7528 E-6 \\
\hline 62.86 & -10.29 & 13.27 & 0.7168 E-6 \\
\hline 63.16 & -13.77 & 15.64 & 0.7226 E-6 \\
\hline 63.46 & -4.30 & 8.95 & 0.7998 E-6 \\
\hline 63.76 & -4.82 & -12.58 & 0.1221 E-S \\
\hline 64.06 & -31.03 & -21.57 & 0.8960 E- 6 \\
\hline 64.36 & 0.99 & 26.70 & 0.1221 E- 5 \\
\hline 65.26 & -19.54 & -14.57 & 0.7907 E-6 \\
\hline 65.56 & -37.93 & -28.08 & 0.1174 E-5 \\
\hline 65.86 & -0.68 & -14.25 & 0.5836 E-6 \\
\hline 66.16 & -8.36 & -12.74 & 0.1132 E- 5 \\
\hline 66.46 & -2.24 & -7.59 & 0.1100 E-5 \\
\hline 66.76 & -40.40 & -16.08 & 0.5730 E-6 \\
\hline 67.06 & -46.99 & -22.00 & 0.9893 E-6 \\
\hline 67.36 & -28.90 & -22.63 & 0.6940 E-6 \\
\hline 67.66 & -40.34 & -24.20 & 0.9952 E-6 \\
\hline 67.96 & -13.94 & 12.94 & 0.2398 E-5 \\
\hline 68.26 & 4.68 & -7.39 & $0.5811 \mathrm{E}-5$ \\
\hline 68.56 & 12.81 & -8.67 & 0.1207 E-4 \\
\hline 68.86 & -15.38 & -31.82 & 0.5909 E-6 \\
\hline 69.16 & -36.63 & -13.25 & 0.1825 E-5 \\
\hline 69.46 & -22.47 & 1.48 & 0.9839 E-6 \\
\hline 69.76 & 10.50 & -4.56 & 0.2184 E-5 \\
\hline 70.06 & 7.62 & -16.42 & 0.1160 E-5 \\
\hline 70.36 & -7.99 & -21.01 & 0.8531 E-6 \\
\hline 70.66 & -7.14 & -17.05 & 0.1001 E-5 \\
\hline 70.96 & -6.28 & -15.04 & 0.1307 E-5 \\
\hline 71.26 & -38.94 & -15.48 & 0.6867 E-6 \\
\hline 71.56 & -0.90 & -0.11 & 0.7850 E-6 \\
\hline 71.96 & -9.45 & -35.85 & 0.9126 E-6 \\
\hline 72.16 & -23.92 & 4.48 & 0.5764 E- 6 \\
\hline 72.46 & 24.06 & 12.24 & 0.4894 E-6 \\
\hline 72.76 & 4.95 & 28.30 & 0.4658 E- 6 \\
\hline 73.06 & -20.15 & 19.87 & 0.4327 E-6 \\
\hline 73.36 & -28.56 & -30.04 & 0.8948 E-6 \\
\hline 74.06 & -90.43 & 0.17 & 0.4064 E- 4 \\
\hline 74.36 & -13.37 & -15.10 & 0.7696 E-6 \\
\hline 74.66 & -29.44 & -12.55 & 0.1080 E- 5 \\
\hline 74.96 & -0.76 & 0.85 & 0.1254 E-5 \\
\hline 75.26 & -13.18 & -12.39 & 0.9283 E-6 \\
\hline 75.86 & -26.76 & -14.11 & 0.1404 E-5 \\
\hline 76.16 & -16.21 & -18.59 & 0.1106 E-5 \\
\hline
\end{tabular}


Appendix B. (Continued).

\begin{tabular}{cccc}
\hline $\begin{array}{c}\text { Sub-bottom } \\
\text { depth } \\
(\mathrm{m})\end{array}$ & $\begin{array}{c}\text { Declination } \\
\left({ }^{\circ}\right)\end{array}$ & $\begin{array}{c}\text { Inclination } \\
\left({ }^{\circ}\right)\end{array}$ & $\begin{array}{c}\text { Intensity } \\
(\mathrm{G})\end{array}$ \\
\hline
\end{tabular}

Hole 575B (Cont.)

\begin{tabular}{|c|c|c|c|}
\hline 76.46 & -10.90 & -22.29 & 0.1367 E-5 \\
\hline 76.76 & -8.00 & -12.59 & 0.1769 E-5 \\
\hline 77.06 & -6.46 & -21.96 & 0.1377 E-5 \\
\hline 77.36 & -13.31 & -9.59 & 0.6332 E- 6 \\
\hline 77.66 & -25.10 & -12.18 & 0.9532 E- \\
\hline 77.96 & -82.82 & 2.57 & 0.1424 E- \\
\hline 78.26 & -17.19 & -14.61 & 0.1169 E-5 \\
\hline 78.56 & 1.01 & -3.93 & 0.1600 E-5 \\
\hline 78.86 & -59.17 & -20.35 & $0.1300 \mathrm{E}-$ \\
\hline 79.16 & -0.80 & -7.88 & $0.1201 \mathrm{E}-$ \\
\hline 79.46 & -21.53 & -14.54 & $0.7287 \mathrm{E}-$ \\
\hline 79.76 & -30.55 & -10.79 & $0.7325 \mathrm{E}-$ \\
\hline 80.06 & -66.67 & 6.52 & 0.1545 E- \\
\hline 80.36 & -34.79 & -14.49 & $0.1739 \mathrm{E}-$ \\
\hline 80.66 & -29.33 & -0.65 & $0.1132 \mathrm{E}-$ \\
\hline 80.96 & -10.81 & 11.35 & $0.1788 \mathrm{E}-$ \\
\hline 81.26 & -11.71 & -13.77 & $0.9595 \mathrm{E}-$ \\
\hline 81.56 & -14.35 & -14.06 & $0.8809 \mathrm{E}-\mathrm{C}$ \\
\hline 81.66 & -18.93 & -10.93 & $0.1317 \mathrm{E}-$ \\
\hline 81.96 & -34.99 & 0.68 & $0.1219 \mathrm{E}-$ \\
\hline 82.86 & -23.84 & 13.90 & $0.1250 \mathrm{E}-$ \\
\hline 83.16 & 19.77 & -6.70 & $0.1522 \mathrm{E}-$ \\
\hline 83.42 & -7.59 & -10.74 & $0.8915 \mathrm{E}-$ \\
\hline 83.76 & 1.75 & -5.61 & $0.1242 \mathrm{E}$ \\
\hline 84.06 & 3.32 & -9.99 & $0.1252 \mathrm{E}$ \\
\hline 84.36 & 35.49 & -0.32 & $0.1812 \mathrm{E}$ \\
\hline 84.66 & 70.37 & 3.80 & $0.7487 \mathrm{E}-$ \\
\hline 84.96 & 57.09 & 4.27 & $0.2568 \mathrm{E}$ \\
\hline 85.26 & 33.18 & 15.33 & $0.1448 \mathrm{E}$ \\
\hline 85.56 & 58.77 & 2.78 & $0.2919 \mathrm{E}-$ \\
\hline 85.86 & 84.94 & 6.36 & $0.2389 \mathrm{E}$ \\
\hline 86.16 & 89.82 & 1.76 & $0.3007 \mathrm{E}$ \\
\hline 86.46 & 91.93 & -7.88 & $0.1799 \mathrm{E}$ \\
\hline 86.76 & 84.18 & 2.16 & $0.2245 \mathrm{E}$ \\
\hline 87.06 & -24.97 & 16.88 & $0.2725 \mathrm{E}$ \\
\hline 87.36 & -71.72 & 9.86 & $0.9756 \mathrm{E}$ \\
\hline 87.66 & -40.84 & 7.16 & $0.4260 \mathrm{E}$ \\
\hline 87.96 & 83.85 & -11.33 & $0.4756 \mathrm{E}$ \\
\hline 88.26 & -22.24 & 25.09 & $0.5686 \mathrm{E}$ \\
\hline 88.56 & 102.97 & 57.54 & $0.3542 \mathrm{E}$ \\
\hline 88.86 & 66.35 & -11.05 & $0.3495 \mathrm{E}$ \\
\hline 89.16 & 32.50 & 8.77 & $0.2541 \mathrm{E}$ \\
\hline 89.46 & -46.89 & 24.29 & $0.7547 \mathrm{E}$ \\
\hline 89.76 & -71.71 & 45.53 & $0.6058 \mathrm{E}$ \\
\hline 90.06 & 18.51 & 19.75 & $0.1815 \mathrm{E}$ \\
\hline 90.36 & 55.82 & 5.41 & $0.6397 \mathrm{E}$ \\
\hline 90.66 & 29.97 & 2.82 & $0.5057 \mathrm{E}$ \\
\hline 91.16 & 146.45 & -7.14 & $0.8780 \mathrm{E}$ \\
\hline 91.46 & 145.40 & 8.99 & $0.7528 \mathrm{E}$ \\
\hline 91.76 & 76.89 & -60.75 & $0.1491 \mathrm{E}$ \\
\hline 92.06 & 127.81 & -17.86 & $0.5163 \mathrm{E}$ \\
\hline 92.36 & 133.17 & -20.26 & $0.6041 \mathrm{E}$ \\
\hline 92.66 & 157.33 & -10.46 & $0.1373 \mathrm{E}$ \\
\hline 92.96 & 157.63 & -4.13 & $0.9955 \mathrm{E}-$ \\
\hline 93.26 & 157.81 & -12.11 & $0.2681 \mathrm{E}$ \\
\hline 93.56 & 155.85 & -13.21 & $0.1977 \mathrm{E}$ \\
\hline 93.86 & -117.09 & -45.19 & $0.4498 \mathrm{E}$ \\
\hline 94.16 & -19.78 & 7.09 & $0.7326 \mathrm{E}$ \\
\hline 94.46 & 132.45 & -6.88 & $0.5738 \mathrm{E}$ \\
\hline 94.76 & -9.05 & 22.01 & $0.2779 \mathrm{E}$ \\
\hline 95.06 & -6.60 & -64.02 & $0.1746 \mathrm{E}$ \\
\hline 95.36 & 144.25 & 19.96 & $0.2065 \mathrm{E}$ \\
\hline 95.66 & -0.46 & 5.15 & $0.2929 \mathrm{E}$ \\
\hline 95.96 & -0.74 & 13.59 & $0.3015 \mathrm{E}$ \\
\hline 96.26 & -10.14 & 11.93 & $0.1482 \mathrm{E}$ \\
\hline 96.56 & -6.51 & 3.07 & $0.2065 \mathrm{E}$ \\
\hline 96.86 & -60.01 & -55.05 & $0.6704 \mathrm{E}$ \\
\hline
\end{tabular}

Appendix B. (Continued).

\begin{tabular}{|c|c|c|c|}
\hline $\begin{array}{l}\text { Sub-bottom } \\
\text { depth } \\
\text { (m) }\end{array}$ & $\begin{array}{c}\text { Declination } \\
\left({ }^{\circ}\right)\end{array}$ & $\begin{array}{c}\text { Inclination } \\
\left({ }^{\circ}\right)\end{array}$ & $\begin{array}{l}\text { Intensity } \\
\text { (G) }\end{array}$ \\
\hline \multicolumn{4}{|c|}{ Hole 575B (Cont.) } \\
\hline 97.16 & -176.90 & -1.03 & 0.6099 E-5 \\
\hline 97.46 & 178.33 & -10.71 & $0.9730 \mathrm{E}-5$ \\
\hline 97.76 & 179.56 & -1.30 & 0.6344 E-5 \\
\hline 98.06 & 86.70 & 5.50 & 0.4404 E-6 \\
\hline 98.36 & -125.74 & -52.16 & $0.5685 \mathrm{E}-5$ \\
\hline 98.96 & 173.29 & 39.86 & $0.8889 \mathrm{E}-6$ \\
\hline 99.26 & 151.86 & 32.10 & $0.1766 \mathrm{E}-5$ \\
\hline 99.86 & -17.13 & -22.42 & 0.1027 E-4 \\
\hline 100.16 & -86.85 & -53.50 & 0.9060 E-6 \\
\hline 100.46 & -9.43 & -7.56 & 0.1923 E-5 \\
\hline 100.76 & -40.98 & -7.20 & $0.1272 \mathrm{E}-4$ \\
\hline 101.06 & 2.43 & -17.90 & 0.1723 E-5 \\
\hline 101.36 & -52.32 & -40.93 & $0.8013 \mathrm{E}-5$ \\
\hline 101.66 & -109.80 & -17.03 & 0.1783 E-5 \\
\hline 101.96 & -35.92 & -3.36 & $0.1620 \mathrm{E}-5$ \\
\hline 102.26 & 2.00 & 4.02 & 0.8173 E-6 \\
\hline 102.56 & -1.35 & -1.32 & 0.2005 E-5 \\
\hline 102.86 & -6.37 & -19.62 & 0.1964 E-5 \\
\hline 103.46 & -56.46 & 1.46 & 0.1908 E-5 \\
\hline 103.76 & -101.92 & -3.87 & $0.1656 \mathrm{E}-5$ \\
\hline 104.06 & -72.82 & -15.17 & 0.2156 E-5 \\
\hline 104.36 & -1.31 & -14.98 & 0.6102 E-6 \\
\hline 104.66 & -2.15 & -0.92 & 0.7449 E- 6 \\
\hline 104.96 & -50.93 & -13.78 & 0.1806 E-5 \\
\hline 105.26 & -4.52 & -12.01 & 0.1153 E-5 \\
\hline 105.56 & -63.75 & -30.20 & 0.1696 E-5 \\
\hline 105.86 & -2.15 & -18.88 & 0.1801 E-5 \\
\hline 106.16 & -28.52 & -20.47 & 0.4574 E-5 \\
\hline 106.46 & 43.37 & -11.14 & 0.8331 E-5 \\
\hline 106.76 & 25.25 & -14.07 & 0.4791 E-5 \\
\hline 107.06 & -53.15 & -28.19 & 0.3274 E-5 \\
\hline 107.36 & -5.72 & -18.47 & 0.9945 E-6 \\
\hline 107.66 & -9.07 & 6.60 & 0.1371 E-5 \\
\hline 108.26 & -17.97 & -19.47 & 0.1213 E-4 \\
\hline 108.86 & -42.15 & 19.83 & 0.3178 E-4 \\
\hline 109.46 & -80.07 & 2.60 & $0.3019 \mathrm{E}-4$ \\
\hline 109.76 & -76.33 & -18.74 & $0.2381 \mathrm{E}-4$ \\
\hline 110.06 & -57.28 & 13.37 & 0.6019 E-5 \\
\hline 110.36 & -91.20 & -19.19 & 0.9835 E-5 \\
\hline 110.66 & -98.53 & -10.65 & 0.6354 E-5 \\
\hline 110.96 & -45.36 & -19.66 & 0.4839 E-5 \\
\hline 111.26 & -82.87 & -24.12 & 0.4936 E-5 \\
\hline 111.56 & -92.97 & -1.40 & 0.3689 E-4 \\
\hline 111.86 & 67.74 & 49.61 & 0.2473 E- 5 \\
\hline 112.46 & 179.73 & 11.33 & $0.1053 \mathrm{E}-4$ \\
\hline 112.76 & 37.84 & 8.25 & 0.2326 E- 5 \\
\hline 113.06 & 46.41 & 7.98 & 0.9392 E-5 \\
\hline 113.36 & 69.82 & 12.65 & 0.2791 E-4 \\
\hline 113.66 & 46.45 & 18.48 & 0.7660 E-5 \\
\hline 113.96 & 71.69 & -0.52 & 0.3150 E-5 \\
\hline 114.42 & 92.58 & -30.47 & 0.7197 E-5 \\
\hline 114.66 & 124.66 & -8.40 & 0.4473 E-5 \\
\hline 114.96 & 159.35 & 34.72 & 0.1882 E-4 \\
\hline 115.26 & 135.74 & 16.43 & 0.1159 E-4 \\
\hline 115.56 & -48.30 & 3.83 & $0.5576 \mathrm{E}-4$ \\
\hline 115.86 & -31.86 & 10.05 & 0.1060 E-4 \\
\hline 116.16 & -35.28 & 28.83 & 0.1309 E-4 \\
\hline 116.46 & -44.92 & 22.00 & $0.4554 \mathrm{E}-5$ \\
\hline 116.76 & -37.86 & 7.38 & $0.6037 \mathrm{E}-5$ \\
\hline 117.06 & 139.31 & -2.87 & $0.1220 \mathrm{E}-4$ \\
\hline 117.36 & 150.60 & 7.96 & 0.1796 E-4 \\
\hline 117.66 & 115.59 & -11.01 & 0.1223 E-5 \\
\hline 117.96 & 84.11 & 15.25 & 0.3027 E-5 \\
\hline 118.26 & -29.03 & 4.87 & 0.4357 E-4 \\
\hline 118.56 & -80.39 & 1.25 & $0.9431 \mathrm{E}-4$ \\
\hline 118.86 & -43.96 & 0.99 & 0.1959 E-4 \\
\hline
\end{tabular}

